\title{
Wall Modeled Large-Eddy Simulations in Rotating Systems for Applications to Turbine Blade Internal Cooling
}

\author{
Keun Min Song \\ A thesis submitted to the faculty of the Virginia Polytechnic Institute and State \\ University in partial fulfillment of the requirements for the degree of \\ Master of Science \\ in \\ Mechanical Engineering
}

Danesh K. Tafti, Chair

Srinath Ekkad

Mark R. Paul

January 20, 2011

Blacksburg, Virginia, USA

Keywords: Heat transfer, Large Eddy Simulation, Rotation, Turbine blade internal cooling, Wall layer modeling

Keun Min Song (C 2011 


\title{
Wall Modeled Large-Eddy Simulations in Rotating Systems for Applications to Turbine Blade Internal Cooling
}

Keun Min Song

\begin{abstract}
Large-Eddy Simulations (LES or wall-resolved LES, WRLES) has been used extensively in capturing the physics of anisotropic turbulent flows. However, near wall turbulent scales in the inner layer in wall bounded flows makes it unfeasible for large Reynolds numbers due to grid requirements. This study evaluates the use of a wall model for LES (WMLES) on a channel with rotation at $R e_{b}=34,000$ from $R o_{b}=0$ to 0.38 , non-staggered $90^{\circ}$ ribbed duct with rotation at $R e_{b}=20,000$ from $R o_{b}=0$ to 0.70 , stationary $45^{\circ}$ staggered ribbed duct at $R e_{b}=49,000$, and two-pass smooth duct with a Ubend at $R e_{b}=25,000$ for $R o_{b}=0$ to 0.238 against WRLES and experimental data. In addition, for the two-pass smooth duct with a U-bend simulations, the synthetic eddy method (SEM) is used to artificially generate eddies at the inlet based on given flow characteristics.

It is presented that WMLES captures the effects of Coriolis forces and predicts mean heat transfer augmentation ratios reasonably well for all simulations. The alleviated grid resolution for these simulations indicates significant reductions in resources, specifically, by a factor of $10-20$ in non-staggered $90^{\circ}$ ribbed duct simulations. The combined effects of density ratio, Coriolis forces, with SEM for the inlet turbulence, capture the general trends in heat transfer in and after the bend.
\end{abstract}


Dedicated to my family

(Dea Han Song, Gue Hee Song, and Keun Hyung (Brian) Song) 


\section{Acknowledgments}

First, I want to thank my advisor, Dr. Danesh Tafti, for his support. I am very grateful that I had an opportunity to expand my knowledge of CFD and applications to internal cooling. I would also like to thank my committee members, Dr. Srinath Ekkad and Dr. Mark Paul, for reviewing my research.

I would like to thank Dr. Tom Diller and Dr. Al Kornhauser for considering me as a graduate teaching assistant for 2 fall semesters for ME 4124, or CAD of Thermal/Fluid Systems. I would also like to thank Dr. Scott Huxtable and Dr. Kevin Kochersberger for considering me as a graduate teaching assistant for ME 4005, or Mechanical Engineering Lab. I am very thankful for 3 semesters of supports from Dr. Mary Kasarda and Dr. Clinton Dancey. I learned a lot from teaching hundreds of undergraduate students (class of 2011 and class of 2012 mechanical engineering students) and it was very fun. I will miss it.

The presented research would not be here today without Dr. Sunil Patil's support who developed WMLES in GenIDLEST. You taught me many things about CFD since I joined HPCFD lab and helped my academic and research work. I also want to thank my lab mates (Adam, Amit, Kamal, Nagendra, Naresh, Sukhjinder, Surya, Vivek K., and Vivek S.) for helping me countless times.

Last but not least, the support of NICS Nautilus is gratefully acknowledged. I would not be able to obtain many of these results without your help. 


\section{Table of Contents}

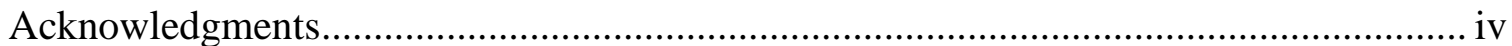

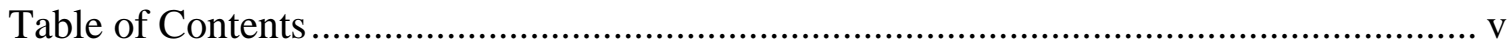

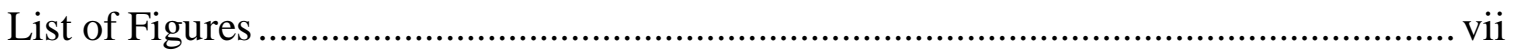

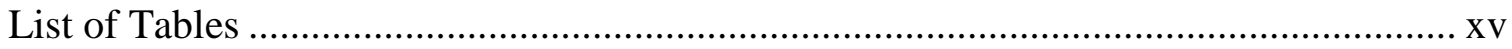

Chapter 1 Background, Motivation, and Objectives ........................................... 1

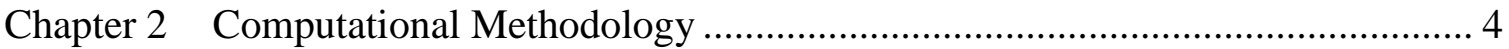

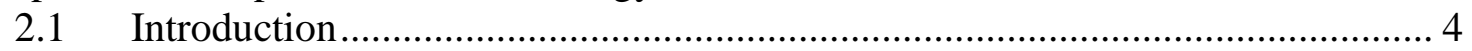

2.2 General Governing Equations ................................................................. 4

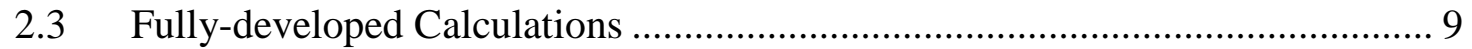

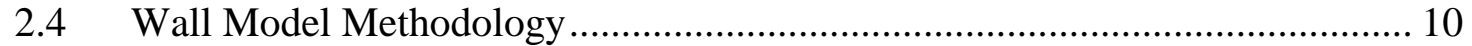

2.5 Synthetic Eddy Methodology .................................................................... 13

2.6 Figures..................................................................................... 14

Chapter 3 Validation of Wall Modeled Large-Eddy Simulations of Turbulent Channel

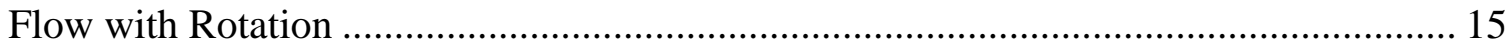

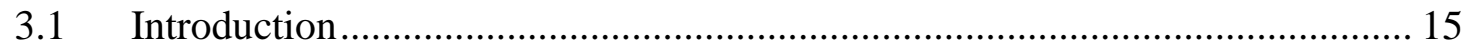

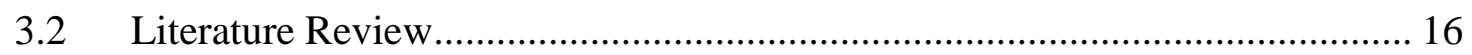

3.3 Computational Model and Details ................................................................ 20

3.4 Validation of Wall Modeled LES of Turbulent Channel Flow....................... 22

3.5 Results of Turbulent Channel Flow with Rotation at $\mathrm{Re}=34,000 \ldots \ldots \ldots \ldots \ldots \ldots . .23$

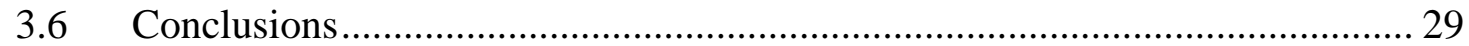

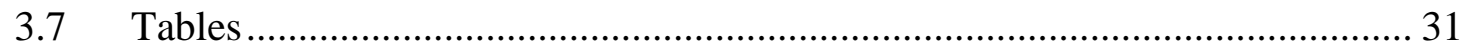

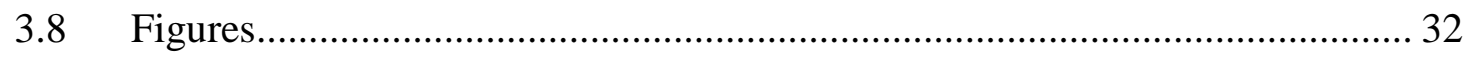

Chapter 4 Wall Modeled Large-Eddy Simulations of $90^{\circ}$ Non-Staggered Ribbed Duct

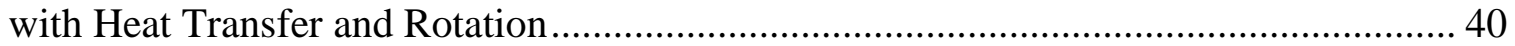

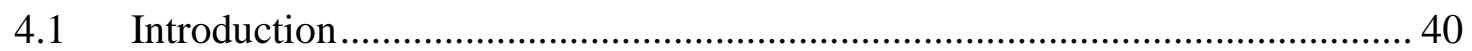

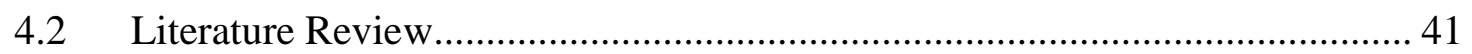

4.3 Computational Model and Details ............................................................ 45

4.4 Validation of Wall Modeled LES of $90^{\circ}$ Non-Staggered Ribbed Duct ........... 47

4.5 Results of $90^{\circ}$ Ribbed Duct with Rotation at $\operatorname{Re}=20,000$......................... 50

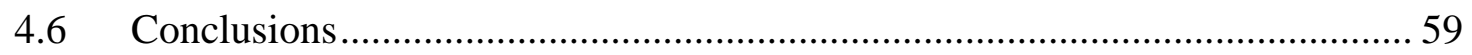

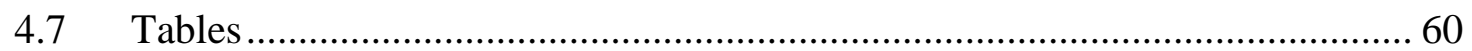

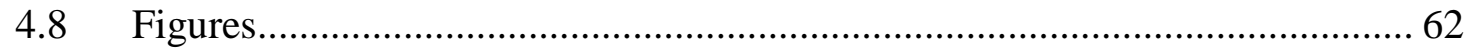

Chapter 5 Wall Modeled Large-Eddy Simulations of $45^{\circ}$ Staggered Ribbed Duct with

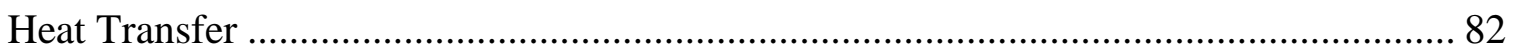

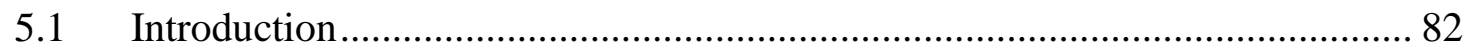

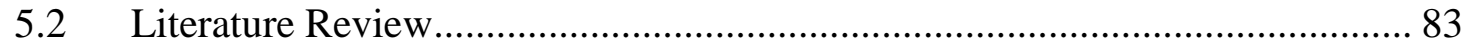

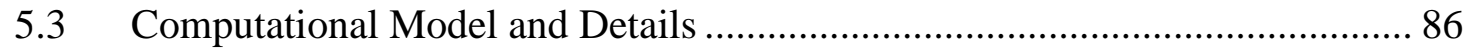

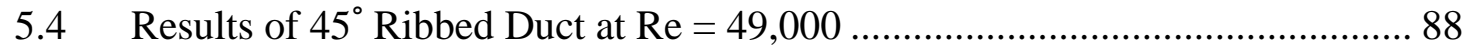

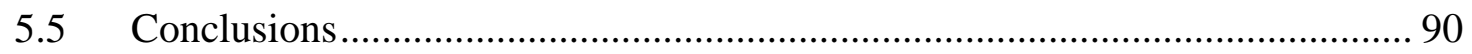

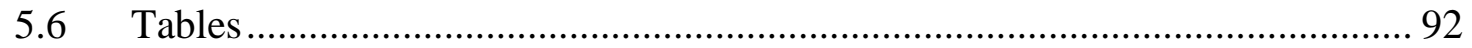

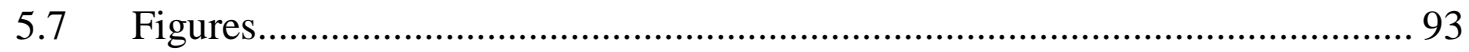


Chapter 6 Wall Modeled Large-Eddy Simulations of Two-pass Smooth Duct with U-

Bend, Heat Transfer, Rotation, and Centrifugal Buoyancy ...................................... 101

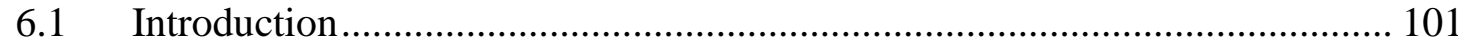

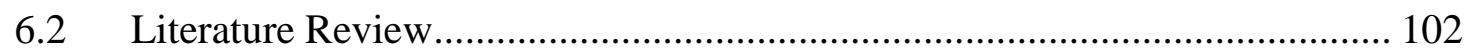

6.3 Computational Model and Details of Fully-developed Smooth Duct Flow ... 106

6.4 Computational Model and Details of Developing Smooth Duct with U-bend108

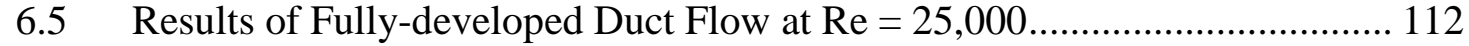

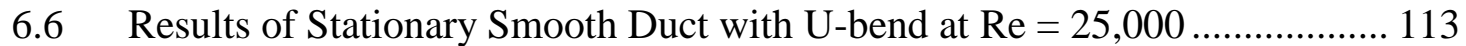

6.7 Results of Rotating Smooth Duct with U-bend at $\mathrm{Re}=25,000$ and $\mathrm{Ro}=0.238$ 118

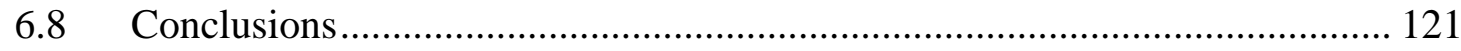

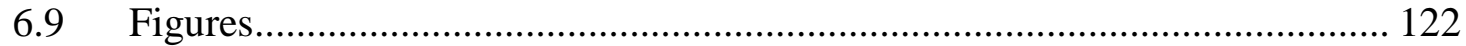

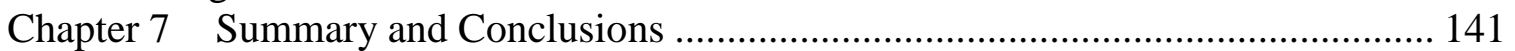

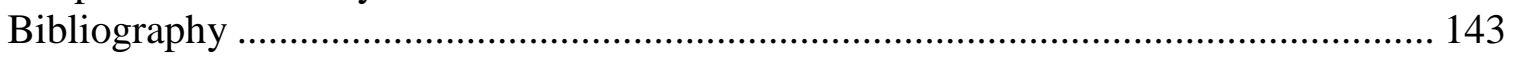




\section{List of Figures}

Figure 2.1 Virtual grid points, or WMLES grid points, are shown to illustrate where a simplified set of equations are solved.

Figure 3.1 The computational domain for turbulent channel flow $(2 \pi \times 2 \times 2 \pi$ in $x, y$, and $\mathrm{z}$ directions respectively).

Figure 3.2 Evolution of volumetric flow rates with non-dimensional time unit for cases

with the near wall treatment and without the near wall treatment.

Figure 3.3 The mean velocity profile from WMLES against the experimental data by

Johnston et al. [13] for Ro $=0$.

Figure 3.4 The mean velocity profile from WMLES against the experimental data by

Johnston et al. [13] for approximately Ro $=0.070$.

Figure 3.5 The mean velocity profile from WMLES against the experimental data by

Johnston et al. [13] for approximately Ro $=0.40$. 34

Figure 3.6 The mean velocity profiles of all rotation numbers.

Figure 3.7 The spanwise roll cells for different rotation numbers at bulk Reynolds

number at 34,000. Arrows indicate directions of the flow: (a) $\operatorname{Ro}=0.070$ (b) $\operatorname{Ro}=0.21$ (c)

Ro $=0.38$

Figure $3.8 \boldsymbol{u}^{\prime} \boldsymbol{u}^{\prime}$ over all rotation numbers. 36

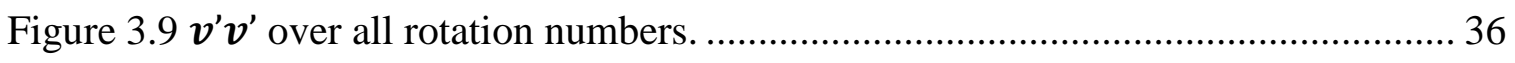

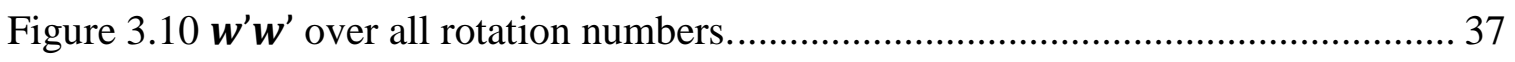

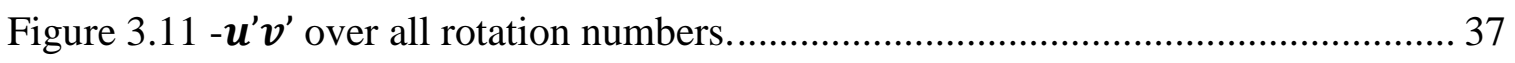

Figure 3.12 Turbulent kinetic energy of all rotation numbers. .................................. 38 
Figure 3.13 The wall friction velocity ratio plot over a range of rotation numbers is plotted where wall friction velocity is normalized by the stationary value at an equivalent bulk Reynolds number. The WMLES result is presented in $\quad$. - - - is the behavior expected from Johnston et al. [13] when both wall layer are turbulent. - $\cdot-\cdots$ behavior when the stable side becomes transitional wall layer. Johnston et al. [13] presented results of a series of bulk Reynolds number where bulk Reynolds number of 10,300 and 11,400 data points are based on wall slope and law of the wall methods whereas other data points from different bulk Reynolds number are from Preston-tube method. Detailed methods of experimental apparatus can be found in Johnston et al. [13].

Figure 4.1 The computational domain for ribbed duct.

Figure 4.2 Volumetric flow rates over a non-dimensional time unit for cases with the near wall treatment and without the near wall treatment.

Figure 4.3 Mean Nusselt values over a non-dimensional time unit for cases with the near wall treatment and without the near wall treatment for different walls. Leading and trailing walls are averaged along with both smooth walls, separately.

Figure 4.4 Mean streamwise velocity distribution along streamwise direction at $y / e=0.1$

and $z=0.5$.

Figure 4.5 Mean wall-normal velocity distribution along streamwise direction at $y / e=0.1$ and $z=0.5$. 64 Figure 4.6 Turbulent profiles at center plane $(z=0.5)$ for a bottom half of the domain at $x / e=4.5$ downstream of the rib. 64

Figure 4.7 Mean flow streamlines of the stationary ribbed duct case. 65 
Figure 4.8 Mean flow streamlines of different rotation numbers at a center plane $(z=0.5)$ :

(a) $\operatorname{Ro}=0.09$ (b) $\operatorname{Ro}=0.17$ (c) $\operatorname{Ro}=0.36$ (d) $\operatorname{Ro}=0.70$ 66

Figure 4.9 Mean flow streamlines of WMLES and LES at a center plane $(z=0.5)$ : (a) Ro $=0.36$ WMLES $(\mathrm{b})$ Ro $=0.35$ LES by Abdel-Wahab [25].

Figure 4.10 Mean wall-normal and spanwise velocities in two symmetric halves of the ribbed duct cross-section for $\mathrm{Ro}=0.36$ at a rib center $(x=0.5)$.

Figure 4.11 Turbulent Reynolds stresses contour plots at a center plane of the ribbed duct $(z=0.5)$ for Ro $=0.36$ (Ro = 0.35 for LES): (a) urms of WMLES (b) urms of LES (c) vrms of WMLES (d) vrms of LES. 68

Figure 4.12 Turbulent Reynolds stresses contour plots at a center plane of the ribbed duct $(z=0.5)$ for Ro $=0.36(\mathrm{Ro}=0.35$ for LES): (a) wrms of WMLES (b) wrms of LES (c)

$\mathbf{u}^{\prime} \mathbf{v}^{\prime}$ of WMLES (d) $\mathbf{u}^{\prime} \mathbf{v}^{\prime}$ of LES.

Figure 4.13 Turbulent kinetic energy (TKE) contours of different rotation numbers at a center plane $(z=0.5)$ : (a) Ro $=0$, (b) Ro $=0.09$ 70 Figure 4.14 Turbulent kinetic energy (TKE) contours of different rotation numbers at a center plane $(z=0.5)$. Left column of contours (a, c, and e) are WMLES results whereas right column of contours (b, d, and f) are LES results. 71 Figure 4.15 Turbulent kinetic energy (TKE) comparison plots of different rotation numbers at $x=1.0$ and $z=0.5$ : (a) WMLES results (b) LES results by Abdel-Wahab [25].

Figure 4.16 Mean Nusselt contour of half domain for the stationary case (leading and trailing sides averaged). The flow moves from left to right. (a) WMLES Ro $=0$ (b) LES Ro $=0$ (c) WMLES Ro $=0.09$ Leading $(d)$ Ro $=0.09$ Trailing. 73 
Figure 4.17 Mean Nusselt contour of half domain for different rotation numbers on leading sides. The flow moves from left to right. Left column of contours (a, c, and e) are WMLES results whereas right column of contours (b, $d$, and $\mathrm{f}$ ) are LES results. 74

Figure 4.18 Mean Nusselt contour of half domain for different rotation numbers on trailing sides. The flow moves from left to right. Left column of contours (a, c, and e) are WMLES results whereas right column of contours (b, $d$, and f) are LES results. 75 Figure 4.19 Mean Nusselt variation on the trailing side superimposed with mean flow streamlines against LES mean Nusselt variation on the trailing side by Abdel-Wahab [25] ( $z=0.5$ for mean flow streamlines). Figure 4.20 Mean Nusselt contour of a half domain on smooth wall: (a) WMLES Ro = 0 (b) LES Ro $=0$. (c) WMLES Ro $=0.09$. 77 Figure 4.21 Mean augmentation contours for all rotation numbers on smooth walls: Left column (a), (c), and (e) are WMLES, right column (b), (d), and (f) are LES. 78 Figure 4.22 Mean Nusselt number contours split into two halves for Ro $=0.36$ on the leading side: (a) WMLES (b) LES.

Figure 4.23 Mean Nusselt number contours split into two halves for Ro $=0.36$ on the leading side: (a) WMLES (b) LES

Figure 4.24 A comparison plot of WMLES, LES by Abdel-Wahab [25], and experimental data by Wagner et al. [30] for heat transfer augmentation ratios for different rotation numbers.

Figure 5.1 The computational domain for $45^{\circ}$ ribbed duct. 93 
Figure 5.2 Mean flow streamlines superimposed on the average surface Nusselt number contours: (a) WMLES at $\mathrm{Re}=49,000$ where flow moves from right to left (b) LES by Abdel-Wahab [25] at $\operatorname{Re}=47,000$ where flow moves from left to right.

Figure 5.3 Mean secondary flow in a plane parallel to ribs at a half pitch: (a) WMLES at $\mathrm{Re}=49,000$ (b) LES by Abdel-Wahab [25] at $\mathrm{Re}=47,000$ (c) Chanteloup et al. [33] at $\operatorname{Re}=50,000$

Figure 5.4 Comparison plot of averaged mean velocity profiles in the center of the duct in a plane cutting through the middle of a top rib $(x=1, z=0.5)$ against LES and experimental data: (a) streamwise velocity profile (b) wall normal velocity profile (c) spanwise velocity profile.

Figure 5.5 Contour plots of turbulent kinetic energy (TKE) and mean flow streamlines at different spanwise locations: (a) $z=0.25$ (b) $z=0.50$ (c) $z=0.75$ 96 Figure 5.6 Turbulent kinetic energy (TKE) at a plane in parallel to ribs (half pitch infront of the top rib): (a) WMLES at $\operatorname{Re}=49,000$ (b) LES by Abdel-Wahab [25] at $\operatorname{Re}=47,000$.

Figure 5.7 Turbulent kinetic energy (TKE) at a plane in parallel to ribs (half pitch behind of the top rib): (a) WMLES at $\operatorname{Re}=49,000$ (b) LES by Abdel-Wahab [25] at $\operatorname{Re}=47,000$.

Figure 5.8 Mean turbulent shear stress, $\boldsymbol{u}^{\prime} \boldsymbol{v}^{\prime}$, at different spanwise locations: (a) $z=0.25$

(b) $z=0.50$ (c) $z=0.75$.

Figure 5.9 Averaged heat transfer augmentation ratios on a ribbed wall and outer wall: (a) WMLES at $\operatorname{Re}=49,000$ (b) Chanteloup et al. [33] at $\operatorname{Re}=50,000$. 100 
Figure 5.10 Averaged heat transfer augmentation ratios on a inner wall: (a) WMLES at

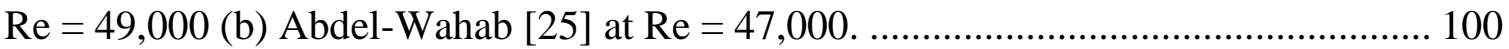

Figure 6.1 The computational domain for the fully-developed smooth duct flow........ 122

Figure 6.2 (a) The computational domain for the two-pass smooth duct with a U-bend (b)

The cross-sectional view of the computational domain where 1 denotes the first pass and

2 denotes the second pass.

Figure 6.3 The mean velocity profiles of WMLES, experiment, and WRLES, respectively.

Figure 6.4 The streamwise velocity contour and secondary flow motion superimposed using contours and vectors: (a) WMLES (b) Contours of streamwise velocity by Madabhushi and Vanka [39] (c) Vector field of secondary flow motion by Madabhushi

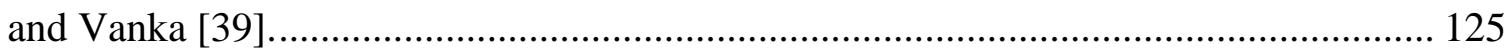

Figure 6.5 The urms profile of WMLES and experiment..................................... 126 Figure 6.6 Variations of Reynolds stresses at the first pass inlet for a stationary fullydeveloped, 500 eddies at a density ratio of 0 , and 1,000 eddies at a density ratio of 0: (a)

urms (b) vrms (c) wrms (d) $u^{\prime} v^{\prime}$

Figure 6.7 Spanwise variations of mean velocity profiles for stationary cases: (a) $x=12$ first pass (b) entrance of the bend (c) center of the bend (d) end of the bend (e) $x=3.1$ second pass 128

Figure 6.8 The variation of turbulent profiles for stationary cases at $x=12$ (first pass). 500 eddies, 1,000 eddies, and experimental data are shown: (a) urms along y-direction (b) wrms along y-direction (c) urms along z-direction (d) wrms along z-direction. 129 
Figure 6.9 The secondary flow motion of a stationary case with 500 eddies and a density ratio of 0 in the middle of the bend $(z=2.05)$ : (a) WMLES (b) Liou and Chen [40]... 130 Figure 6.10 The variation of $\boldsymbol{u r m s}$ in two-passes for a stationary periodic, 500 eddies at a density ratio of 0 and 1,000 eddies at a density ratio of 0: (a) Entrance of the bend (b) Center of the bend (c) Exit of the bend (d) $x=3.1$ second pass.

Figure 6.11 The variation of wrms in two-passes for a stationary periodic, 500 eddies at a density ratio of 0 and 1,000 eddies at a density ratio of 0: (a) Entrance of the bend (b) Center of the bend (c) Exit of the bend (d) $x=3.1$ second pass.

Figure 6.12 Averaged heat transfer augmentation ratio contour plots on all 4 walls at a density ratio of 0.13 for a stationary simulation: (a) Leading wall (b) Trailing wall (c) Outer wall (d) Inner wall. 133

Figure 6.13 Heat transfer augmentation ratio variations of two-pass smooth duct with Ubend for a stationary case: (a) leading side (b) trailing side (c) outer wall (d) inner wall.

Figure 6.14 The streamlines at $z=0.25$ : (a) a density ratio of 0 (b) a density ratio of 0.13 . 135

Figure 6.15 The streamlines at different locations for a density ratio of 0 at $y=0.06 \ldots 136$ Figure 6.16 Wall-normal variations of streamwise velocity profiles for rotational cases at different locations for 500 eddies: (a) $x=12$ first pass (b) entrance of the bend (c) center of the bend (d) end of the bend (e) $x=3.1$ second pass

Figure 6.17 Spanwise variations of streamwise velocity profiles for rotational cases at different locations for 500 eddies: (a) $x=12$ first pass (b) entrance of the bend (c) center of the bend (d) end of the bend (e) $x=3.1$ second pass...... 138 
Figure 6.18 The secondary flow motion of 500 eddies with a density ratio of 0 in the middle of the bend $(z=2.05)$ : (a) a bulk rotation number of 0.238 with WMLES (b) a

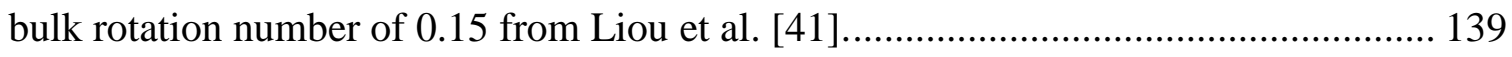
Figure 6.19 Heat transfer augmentation ratio variations of two-pass smooth duct with Ubend for a bulk rotation number of 0.238: (a) leading side (b) trailing side (c) outer wall

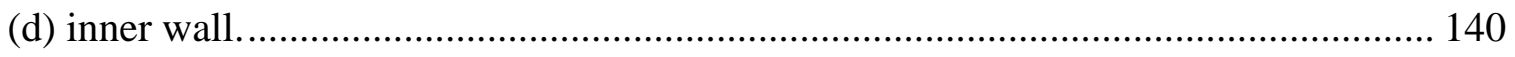




\section{List of Tables}

Table 3.1 A summary of referenced data used to compare present study.

Table 4.1 The summary of ribbed duct calculation with and without wall treatment

against wall resolved LES by Tafti [23] and experimental data by Rau et al. [24]....

Table 4.2 The summary of ribbed duct calculation of all rotation cases. LES results by Tafti [23] are used for the stationary case and LES results by Abdel-Wahab [25] for nonzero rotation cases.

Table 5.1 The summary of ribbed duct calculation with and without wall treatment against wall resolved LES by Abdel-Wahab [25] and experimental data by Chanteloup et

al. [33] 


\section{Chapter 1 Background, Motivation, and Objectives}

High demands for more efficient and high performance output from rotating machinery required researchers to study highly turbulent flows. Due to these high demands, turbine blade materials are often operated close to their temperature limits to meet performance needs. In order to understand the physics at high Reynolds numbers, computational fluid dynamics (CFD) is used to simulate flow and heat transfer at the desired conditions. Despite the fact that high performance computing has come a long way, the resources required to solve the Navier-Stokes equations at high Reynolds number by using Direct Numerical Simulations (DNS) are impossibly large. In an effort to capture much of the physics in highly turbulent flows, Large-Eddy Simulations (LES) has been used to simulate this type of flows, which requires less computational resources than DNS. Even then, the grid resolution requirement for wall-bounded LES is still resource intensive.

In order to alleviate the computational load for LES, Patil and Tafti [1] developed a wall-layer model. Initial studies of the near wall treatment significantly reduced the amount of resources required to simulate highly turbulent flows without much loss in accuracy as reported in Patil [2]. This thesis tests the wall modeling in rotating systems to investigate whether the model treatment of the inner layer adequately captures the stabilizing-destabilizing effect of Coriolis forces on near wall turbulence. For this purpose, a turbulent channel flow, a ribbed duct, and a smooth duct with a 180 degree bend are investigated under rotating conditions. The wall model is also investigated for 
property variation effects which lead to centrifugal buoyancy forces in the 180 degree duct case.

The thesis is organized as follows:

- Governing equations and relevant terms are described in Chapter 2 for both developing and fully-developed calculations. In addition to LES methodologies, the wall model methodology is briefly described to give an overview of how calculations were carried out.

- In Chapter 3, a stationary turbulent channel flow is validated with WMLES against WRLES at $\operatorname{Re}=34,000$. Furthermore, rotation numbers up to $\mathrm{Ro}=0.38$ are investigated to observe the accuracy of shear stress calculations based on experimental and computational data.

- Chapter 4 presents results for a 90 degree ribbed duct at $\mathrm{Re}=20,000$ with rotation numbers up to Ro $=0.70$. A part of this chapter is published as a conference paper (Song, K. and Tafti, D.K., 2011. "WALL MODELED LARGE-EDDY SIMULATIONS IN ROTATING SYSTEMS FOR APPLICATIONS TO TURBINE BLADE INTERNAL COOLING,” Paper No. ISHMT_USA_014, ISHMT-ASME 2011, Chennai, India.).

- Chapter 5 presents WMLES results of a $45^{\circ}$ staggered ribbed duct with heat transfer. At $\operatorname{Re}=49,000$, the flow behavior is observed against experimental and computational data.

- Chapter 6 investigates a two pass smooth duct with a U-bend at a rotation number of $\mathrm{Ro}=0.238$. Both Coriolis and centrifugal buoyancy effects are tested. The SEM is used to generate turbulent boundary conditions. 
- The thesis is summarized in Chapter 7 with concluding remarks. 


\section{Chapter 2 Computational Methodology}

\subsection{Introduction}

The objective of this chapter is to summarize the governing equations and computational methodology that was used to simulate each case. Section 2.2 gives a general description of the conservation of mass, momentum and energy in vector notation for an essentially incompressible fluid with temperature dependent properties. Section 2.3 follows this by giving a modified form of the equations when a fully-developed assumption is invoked. Section 2.4 gives a description of the wall model used with the LES. Section 2.5 gives a brief description of the Synthetic Eddy Method (SEM), which is used for generating the turbulent inlet conditions.

\subsection{General Governing Equations}

The low speed variable property, grid-filtered, continuum conservation of mass, momentum, and energy equations are described by the following dimensionless timedependent, conservation equations (Tafti [3]).

Mass Conservation:

$\vec{\nabla} \cdot(\rho \vec{u})=0$

Momentum Conservation: 


$$
\begin{aligned}
& \frac{\partial(\rho \vec{u})}{\partial t}+\vec{\nabla} \cdot(\rho \vec{u} \vec{u}) \\
&=-\vec{\nabla} P+\frac{1}{\operatorname{Re}} \vec{\nabla} \cdot(\left(\mu+\mu_{t}\right) \underbrace{\left\{\left(\vec{\nabla} \vec{u}+\vec{\nabla} \vec{u}^{T}\right)-\frac{2}{3} \mu(\vec{\nabla} \cdot \vec{u}) \overrightarrow{\vec{I}}\right\}}_{2 \overrightarrow{\vec{S}^{a}}}) \\
&+\frac{\operatorname{Ra}}{\operatorname{Re}^{2} \operatorname{Pr}} \frac{\rho}{(\Delta T)} \vec{e}_{y}-2 \rho \vec{\omega} \times \vec{u}-\rho \vec{\omega} \times(\vec{\omega} \times \vec{r})+\vec{S}_{u}
\end{aligned}
$$

Energy Conservation:

$$
\frac{\partial(\rho T)}{\partial t}+\vec{\nabla} \cdot(\rho \vec{u} T)=\frac{1}{\operatorname{Re} \operatorname{Pr}} \vec{\nabla} \cdot\left(\left(\kappa+\kappa_{t}\right) \vec{\nabla} T\right)+S_{\theta}
$$

where

$$
\begin{aligned}
& \rho=\frac{\rho^{*}}{\rho_{r e f}^{*}} ; \mu=\frac{\mu^{*}}{\mu_{r e f}^{*}} ; \kappa=\frac{\kappa^{*}}{\kappa_{r e f}^{*}} ; c c_{p}=\frac{c_{p}^{*}}{c_{p_{-} r e f}^{*}} ; \vec{x}=\frac{\vec{x}^{*}}{L_{r e f}^{*}} ; \vec{u}=\frac{\vec{u}^{*}}{U_{r e f}^{*}} ; t=\frac{t^{*} U_{r e f}^{*}}{L_{r e f}^{*}} ; \\
& P=\frac{P^{*}-P_{r e f}^{*}}{\rho_{r e f}^{*} U_{r e f}^{* 2}} \text { and } T=\frac{T^{*}-T_{r e f}^{*}}{T_{o}^{*}}
\end{aligned}
$$

The notation * is used for dimensional quantities and ref is for reference quantities where $\vec{\nabla}$ is the gradient operator, $\vec{\omega}$ is the non-dimensional angular velocity vector. An equivalent Rotation number defined in equation (2.5) based on the z-component of the angular velocity vector. $\rho$ is density, $c_{p}$ is specific heat capacity, $\vec{x}$ is Cartesian length vector, $\vec{u}$ is Cartesian velocity vector, and $t$ is time. The reference temperature, $T_{r e f}^{*}$ and pressure $P_{r e f}^{*}$ are used for calculating all reference property values, whereas $T_{o}{ }^{*}$ is the non-dimensionalizing temperature scale. The dynamic viscosity $\mu^{*} / \mu_{r e f}^{*}$ and thermal 
conductivity $\kappa^{*} / \kappa_{\text {ref }}^{*}$ variations with temperature are represented by Sutherland's law for gases (White [4]). While these two quantities vary substantially with temperature, specific heat has a much weaker dependence. For example, viscosity and conductivity for air in the temperature range $240 \mathrm{~K}$ to $960 \mathrm{~K}$ vary by more than $100 \%$, whereas $c_{p}{ }^{*}$ only varies $12 \%$. Hence, it is assumed that $c_{p}^{*} / c_{p_{-} r e f}^{*}=1$, and that the gravitational vector $\vec{g}^{*}=g^{*} \vec{e}_{y}$. Velocity and temperature are Favre filtered quantities, and $\vec{S}_{u}$ and $S_{\theta}$ are source terms which are used to accommodate additional modifications to the governing equations, such as periodicity in the flow direction, heat flux boundary conditions, and the use of turbulent wall treatments. $\overrightarrow{\vec{I}}$ is the unit matrix and $\overrightarrow{\vec{S}}^{a}$ is the strain rate tensor expressed as shown in equation (2.2). The last two terms in equation (2.2), excluding $\vec{S}_{u}$ term, express the non-inertial effects of coordinate frame rotation: the first term representing the Coriolis forces, and the second term representing the centrifugal force terms; $\vec{r}$ is the radial vector from the axis of rotation. The characteristic non-dimensional parameters, Reynolds number $(R e)$, Prandtl number $(P r)$, Rayleigh number $(R a)$, and Rotation number $(R o)$, which is the inverse of Rossby number, take the following form:

$\operatorname{Re}=\frac{\rho_{r e f}^{*} U_{r e f}^{*} L_{r e f}^{*}}{\mu_{r e f}^{*}}, \operatorname{Pr}=\frac{\mu_{r e f}^{*} c_{p_{-} r e f}^{*}}{\kappa_{r e f}^{*}}, \operatorname{Ra}=\frac{g^{*} \beta^{*}\left(\Delta T^{*}\right) L_{r e f}^{*}}{\alpha_{r e f}^{*} v_{r e f}^{*}}, \operatorname{Ro}=\omega=\frac{\omega^{*} L_{r e f}^{*}}{U_{r e f}^{*}}$

where $\alpha_{r e f}^{*}$ is the thermal diffusivity and $v_{r e f}^{*}$ is the kinematic viscosity. Here, $\beta^{*}$ is the thermal expansion coefficient and is approximated as $1 / T_{r e f}^{*}$ for an ideal gas. Reynolds number indicates the ratio of the inertial force to the viscous force. Prandtl number indicates the ratio of viscous diffusion rate and thermal diffusion rate. $R a /\left(R e^{2} P r\right)$ in 
equation (2.2) signifies the relative importance of forced convection versus natural convection. The above three equations, excluding Rotation number, are augmented by the equation of state, which assumes an ideal gas and is used to calculate the density:

Equation of State:

$\rho=\frac{\rho_{r e f}{ }^{*} U_{r e f}{ }^{* 2} P+P_{r e f}{ }^{*}}{R^{*} \rho_{r e f}{ }^{*}\left(T \cdot T_{o}{ }^{*}+T_{r e f}{ }^{*}\right)}$

where $R^{*}$ is the gas constant. The subgrid turbulent viscosity, $\mu_{t}$, is modeled by the Smagorinsky model (Smagorinsky [5]) as follows:

$\mu_{t}=\rho C_{s}^{2} \Delta_{g}^{2}\left|\bar{S}^{a}\right|$

where $C_{S}^{2}$ is the Smagorinsky model constant, $\Delta_{g}$ is the average grid length scale associated with the computational cell, and $\left|S^{a}\right|$ is the magnitude of the anisotropic part of the strain rate tensor given by $\left|S^{a}\right|=\sqrt{2 S_{i k} S_{i k}}$. The Smagorinsky model constant is found by using the dynamic procedure (Germano et al. [6], Lilly [7], Najjar and Tafti [8]) by applying a second test filter, $\hat{G}$, is applied to the filtered governing equations with the characteristic length scale of the filter larger than the grid filter, $\bar{G}$. In order to obtain the test filtered quantity from the grid filtered quantity a second-order trapezoidal filter in one dimension is used:

$\hat{\varphi}=\frac{1}{4}\left(\bar{\varphi}_{i-1}+2 \bar{\varphi}_{i}+\bar{\varphi}_{i+1}\right)$

where $\varphi$ is the variable of the interest such as each velocity component, $\mathrm{u}, \mathrm{v}$, and $\mathrm{w}$. Consequently, the subgrid stresses given by equation (2.8) are modeled as follow:

$\tau_{i j}^{a}=\left(\overline{\rho u_{i} u_{j}}-\bar{\rho} \overline{u_{i}} \overline{u_{j}}\right)=-2 \bar{\rho} C_{s}^{2} \Delta_{g}^{2}\left|\overline{S^{a}}\right| \overline{S_{i j}^{a}}$ 
where the overbar denotes grid-filtered quantities. Similarly, the sub-test stresses are modeled by the Smagorinsky model as:

$T_{i j}^{a}=\left(\widehat{\overline{\rho u_{l} u_{J}}}-\hat{\bar{\rho}} \widehat{\overline{u_{l}}} \widehat{\overline{u_{J}}}\right)=-2 \hat{\bar{\rho}} C_{s}^{2} \Delta_{t}^{2}\left|\widehat{\overline{S^{a}}}\right| \widehat{S_{l \jmath}^{a}}$

where $\Delta_{t}$ is the length scale associated with the test filter, and ${ }^{-}$denotes a test filtered quantity. By applying the test filter to the subgrid stresses, the following equation is formulated:

$\widehat{\tau_{l \jmath}^{a}}=\left(\widehat{\overline{\rho u_{l} u_{J}}}-\bar{\rho} \overline{\overline{u_{l}} \overline{u_{J}}}\right)=-2 C_{s}^{2} \Delta_{g}^{2} \bar{\rho} \mid \widehat{\widehat{S^{a}} \mid \overline{S_{l J}^{a}}}$

The resolved turbulent stresses, representing the energy scales between the test and the grid filters, are:

$L_{i j}^{a}=L_{i j}-\frac{1}{3} L_{k k}=T_{i j}^{a}-\widehat{\tau_{l \jmath}^{a}}=\bar{\rho} \widehat{\overline{u_{\imath}}} \overline{u_{\jmath}}-\hat{\bar{\rho}} \widehat{\overline{u_{\imath}}} \widehat{\overline{u_{\jmath}}}$

Hence,

$L_{i j}^{a}=-2 C_{s}^{2} \Delta_{g}^{2}\left[\alpha \hat{\bar{\rho}}\left|\widehat{\overline{S^{a}}}\right| \hat{\bar{S}}_{i j}^{a}-\bar{\rho} \mid \widehat{\overline{S^{a}} \mid} \overline{S_{l j}^{a}}\right]=-2 C_{s}^{2} \Delta_{g}^{2} M_{i j}$

The variable, $\alpha$, is the square of the ratio of the characteristic length scale associated with the test filter to the grid filter and is taken to be $\left[\Delta_{t} / \Delta_{g}=\sqrt{6}\right]$ for a three-dimensional test filtering operation (Najjar and Tafti [8]). Using a least-squares minimization procedure of Lilly [7], a final expression for $C_{s}^{2}$ is obtained:

$C_{s}^{2}=-\frac{1}{2} \frac{1}{\Delta_{g}^{2}} \frac{L_{i j}^{a} M_{i j}}{M_{i j} M_{i j}}$

where $C_{S}^{2}$ is constrained to be positive values to maintain numerical stability.

The turbulent thermal conductivity is calculated as $k_{t}=\mu_{t} / P r_{t}$, where $P r_{t}$ is the turbulent Prandtl number and is assumed to have a constant value of 0.5 according to Moin et al. [9]. 


\subsection{Fully-developed Calculations}

Fully-developed calculations are performed by assuming constant fluid properties and periodic boundary conditions in the flow direction. Invoking the balance between the pressure drop and form and friction losses in the domain of length $L_{x}^{*}$, the reference velocity $U_{r e f}^{*}$ is chosen to be an effective friction velocity given by $U_{r e f}^{*}=u_{\tau}^{*}=\sqrt{\left(-\Delta P_{x}^{*} / L_{x}^{*}\right)\left(D_{h}^{*} / 4 \rho^{*}\right)}$

where $\Delta P_{x}^{*}$ is the mean pressure drop in the flow direction across the computational domain length of $L_{x}^{*}$, and $D_{h}^{*}$ is the hydraulic diameter. The characteristic temperature scale is defined as $T_{o}^{*}=q_{w}^{*} L_{r e f}^{*} / \kappa^{*}$, where $-q_{w}^{*}$ is the applied wall heat flux. Since both pressure and temperature have a $\mathrm{x}$-directional dependence, the assumed periodicity of the domain in the streamwise or $x$-direction requires the mean gradient of pressure and temperature to be isolated from the fluctuating periodic components as follows:

$$
\begin{aligned}
& P^{*}(\vec{x}, t)=P_{\text {ref }}^{*}-\beta^{*} x^{*}+p^{*}(\vec{x}, t) \\
& T^{*}(\vec{x}, t)=T_{\text {ref }}^{*}+\gamma^{*}(t) \cdot x^{*}+\theta^{*}(\vec{x}, t)
\end{aligned}
$$

where $\beta^{*}=-\Delta P_{x}^{*} / L_{x}^{*}$ is the mean pressure gradient, $p^{*}$ is the periodic pressure fluctuations, $\gamma^{*}$ is a time-dependent temperature gradient, and $\theta^{*}$ is the fluctuating or periodic temperature component. Non-dimensionalizing equation (2.16) gives

$$
\begin{aligned}
& P(\vec{x}, t)=-\beta x+p(\vec{x}, t) \\
& T(\vec{x}, t)=\gamma(t) x+\theta(\vec{x}, t)
\end{aligned}
$$

where $\beta=4 / D_{h}$ and $\gamma=q_{w} \Omega / \operatorname{Re} \cdot \operatorname{Pr} \cdot Q_{x} \cdot L_{x}$, where $\Omega$ is the heat transfer surface area and $\mathrm{Q}_{\mathrm{x}}$ is the flowrate. 
Substituting equation (2.17) in the non-dimensional conservation equations (2.1-2.3) gives the following modified momentum and energy equations:

Mass Conservation:

$\vec{\nabla} \cdot \vec{u}=0$

Momentum Conservation:

$$
\frac{\partial \vec{u}}{\partial t}+\vec{\nabla} \cdot(\vec{u} \vec{u})=-\vec{\nabla} p+\vec{\nabla} \cdot\left(\left(\frac{1}{\operatorname{Re}}+\frac{1}{\operatorname{Re}_{\mathrm{t}}}\right) \vec{\nabla} \vec{u}\right)-2 \vec{\omega} \times \vec{u}+g \vec{e}_{y}+\beta \vec{e}_{x}
$$

Energy Conservation:

$\frac{\partial \theta}{\partial t}+\vec{\nabla} \cdot(\vec{u} \theta)=\vec{\nabla} \cdot\left(\left(\frac{1}{\operatorname{Re} \operatorname{Pr}}+\frac{1}{\operatorname{Re}_{\mathrm{t}} \operatorname{Pr}_{\mathrm{t}}}\right) \vec{\nabla} \theta\right)-\mu_{x}$

with modified boundary conditions,

$\phi(x, t)=\phi\left(x+L_{x}, t\right), \phi=\vec{u}, p$ and $\theta$,

and

$-\vec{\nabla} \theta \cdot \vec{n}=-1+\vec{e}_{x} \cdot \vec{n}$

For generalization to complex geometries, equations $(2.18-2.20)$ are mapped from physical coordinates $(\vec{x})$ to logical/computational coordinates $(\vec{\xi})$ by a boundary conforming transformation $\vec{x}=\vec{x}(\vec{\xi})$, where $\vec{x}=(x, y, z)$ and $\vec{\xi}=(\xi, \eta, \zeta)$ (Thompson et al. [10]).

\subsection{Wall Model Methodology}

The wall model solves a simplified set of equations by using a virtual grid along the normal from the first off-wall grid point to the wall (Patil and Tafti [1]) as shown in Figure 2.1. A tangential momentum equation is solved in the inner layer by using the 
outer instantaneous LES velocity at the first off-wall grid point as a boundary condition. The calculated wall shear stress from the inner layer velocity profile is used as the boundary condition in the outer layer calculation to complete the coupling. A reduced form of the tangential momentum equation as given by

$$
\begin{aligned}
& \frac{1}{\operatorname{Re}} \frac{\partial}{\partial n}\left(\left(\mu+\mu_{t}\right) \frac{\partial u_{t}}{\partial n}\right) \\
& =\vec{\nabla} P \cdot \vec{t}-\left[\frac{\operatorname{Ra}}{\operatorname{Re}^{2} \operatorname{Pr}} \frac{\rho}{(\Delta T)} \vec{e}_{y}-2 \rho \vec{\omega} \times \vec{u}-\rho \vec{\omega} \times(\vec{\omega} \times \vec{r})\right] \cdot \vec{t}
\end{aligned}
$$

Equation (2.23) is solved in the inner layer, where $u_{t}$ is the tangential velocity component, $\vec{t}$ is the unit tangent vector to the surface and $n$ is the normal distance from the surface. The component of pressure gradient in the tangential direction is calculated in the outer layer and is assumed to be constant in the inner layer. The non-inertial rotational Coriolis and centrifugal force terms are calculated in the inner layer by assuming fluid properties at the outer edge of the inner layer. Turbulent viscosity in the inner layer is modeled by

$$
\mu_{t}=\kappa \mu d^{+}\left(1-e^{-d^{+} / A}\right)^{2}
$$

where $\kappa$ is Von-Karman constant, $d$ is a normal distance from the wall, $A$ is 19 , and:

$$
\begin{aligned}
& d^{+}=\frac{u_{\tau} d}{v} \\
& u_{\tau}=\sqrt{\frac{\tau_{w}}{\rho}} \\
& \left\|\tau_{w}\right\|=\left.\mu \frac{\partial u_{t}}{\partial n}\right|_{w a l l}
\end{aligned}
$$

The set of equations (2.23-2.27) are solved iteratively in the inner layer by solving a onedimensional tri-diagonal system at each iteration. The resulting wall shear stress in the 
tangential direction (equation 2.27), is transformed back into a $(x, y, z)$ coordinate system to be used as a boundary condition in the outer LES calculation. Details of the procedure can be found in Patil and Tafti [1].

Similarly, a reduced form of the energy equation is also solved in the inner layer as:

$\frac{1}{\operatorname{RePr}} \frac{\partial}{\partial n}\left[\left(k+\frac{\mu_{t}}{\sigma_{t}}\right) \frac{\partial \theta}{\partial n}\right]=0$

The formulation of Kays [11] is used:

$\frac{1}{\sigma_{t}}=0.58+0.22\left(\frac{\mu_{t}}{\mu}\right)-0.0441\left(\frac{\mu_{t}}{\mu}\right)^{2}\left\{1-e^{-5.165 /\left(\frac{\mu_{t}}{\mu}\right)}\right\}$

where this formulation accounts for the higher values of turbulent Prandtl number very close to the wall and gradually decays from the wall according to Patil and Tafti [1].

There are two different boundary conditions that are used in the following chapters: specified constant heat flux, and specified temperature. With the heat flux specified at the wall, the inner layer calculation is used to find the wall temperature, whereas with the temperature specified at the wall, the inner layer calculation is used to find the heat flux at the wall.

The following equation, Equation (2.30), is used to obtain the wall temperature in the inner layer

$T_{\text {wall }}=T_{i 2}+\frac{\Delta d}{k} q_{w}$

where $T_{i 2}$ is the first off-wall node in the inner layer, $\Delta d$ is the distance from the wall, and $q_{w}$ is the heat flux. The calculated wall temperature is used to calculate the heat transfer coefficient. 
Similarly, equation (2.31) below is used to obtain the heat flux at the wall given the wall temperature, based on the calculated temperature profile in the inner region.

$q_{w}=-\left.k \frac{\partial T}{\partial n}\right|_{w a l l}$

The calculated wall heat flux is used as a boundary condition in the outer layer calculation. Detailed methodologies of these processes can be found in Patil [2].

\subsection{Synthetic Eddy Methodology}

The synthetic eddy method (Jarrin et al. [12]) is used to generate inlet turbulence for the developing flow calculation in Chapter 6. Based on the statistic data of velocities, turbulent quantities, and turbulent length scales, either obtained from experiments or simpler precursor simulations, eddies with appropriate characteristics are placed randomly at the inlet in an eddy box. A velocity kernel function is associated with each eddy. These artificial eddies in the eddy box convect based on the mean velocity to ensure that they are correlated in time. As these eddies move out of the eddy box, new eddies are continuously placed in the eddy box based on the specified number of eddies. Inlet turbulence is generated by taking the collective effect of all eddies on the velocity nodes in the inlet plane, conditioned by the target turbulent statistics. The net result is the generation of instantaneous turbulence which is spatially and temporally correlated based on the target integral length scales and the mean velocity profile input into the method. 


\subsection{Figures}

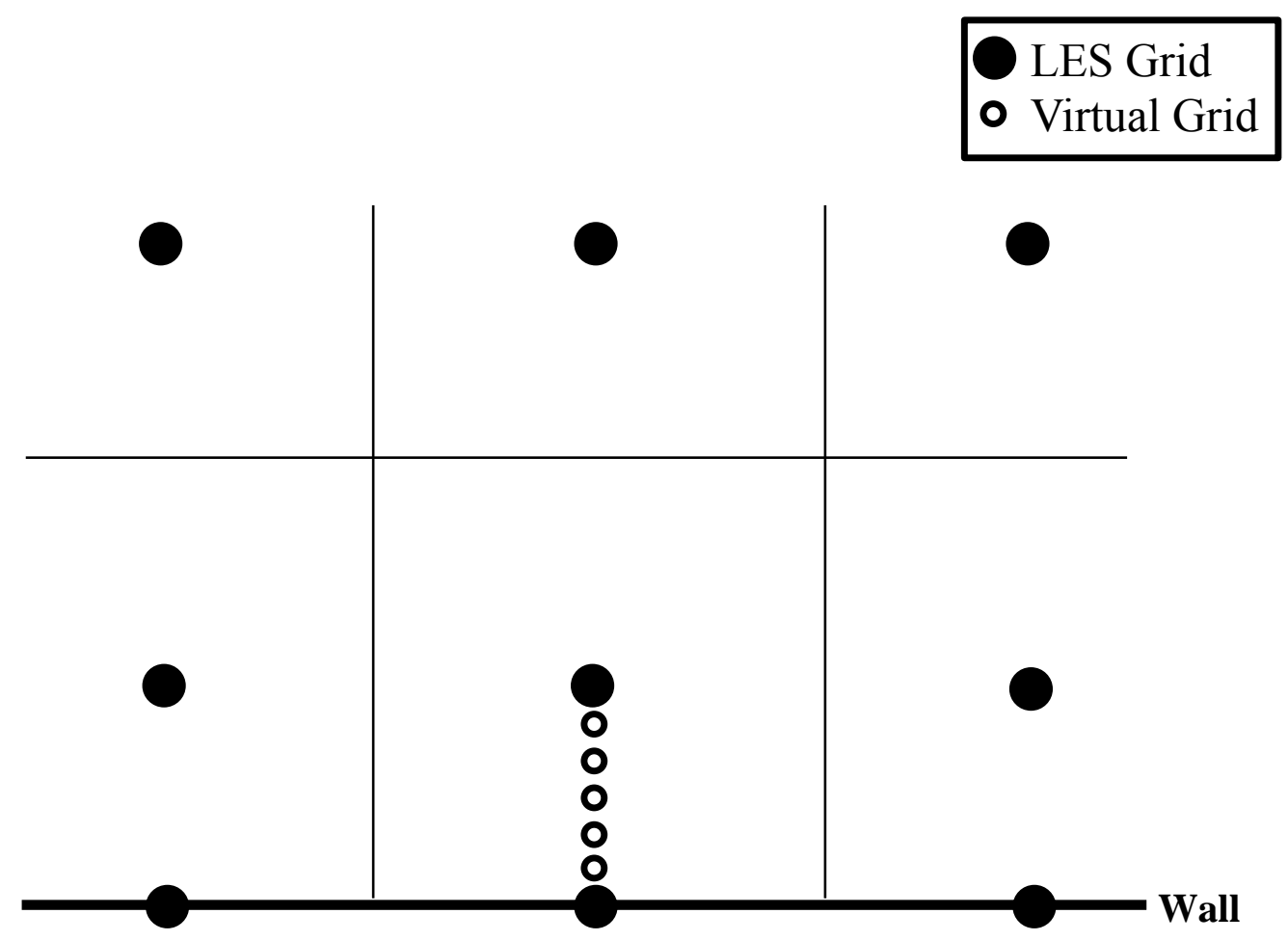

Figure 2.1 Virtual grid points, or WMLES grid points, are shown to illustrate where a simplified set of equations are solved. 


\section{Chapter 3 Validation of Wall Modeled Large-Eddy Simulations of Turbulent Channel Flow with Rotation}

\subsection{Introduction}

Based on methodologies mentioned in the previous chapter, rotating turbulent channel flow with Coriolis forces is examined for investigating the robustness and accuracy of wall model. Compared to the fine grid resolution of LES where $\Delta \mathrm{y}_{1}{ }^{+}<1$, and $\Delta^{+}<20-50$ in the wall parallel directions, the grid requirements for the wall model LES (WMLES) are significantly smaller. The analysis of turbulent channel flow at $R e_{b}$ of 34,000 for $0,0.070,0.10,0.21$, and 0.38 bulk rotation numbers, $R o_{b}$, is presented in this chapter. These conditions were motivated by the experiments of Johnston et al. [13]. This is a first attempt in the literature to simulate a high Reynolds number rotational flow using LES. The results of each simulation was compared with experimental data by Johnston et al. [13] and numerical simulations by Kristoffersen and Andersson [14], Tafti and Vanka [15], Miyake and Kajishima [16], and Piomelli and Scalo [17]. Notably, mean velocity profiles for $R o_{b}=0,0.070$, and 0.38 , turbulent rms quantities for all rotation numbers, turbulent kinetic energy values, friction velocity ratio variations over a range of bulk rotation numbers, and spanwise roll cells were analyzed to observe the flow characteristics.

Coriolis forces in rotating systems lead to flow stabilization and destabilization of turbulence on the trailing and leading sides of the channel by directly impacting the mechanisms of turbulent production via bursts and sweep events of turbulent eddies. Bursts occur due to the movement of low momentum fluid away from the wall whereas 
sweeps occur due to the movement of high momentum fluid toward the wall. Both bursts and sweeps contribute to the production of turbulence. The leading side, which is the stable side or suction side, undergoes laminarization near walls due to the suppression of turbulence production by Coriolis forces. In contrast, the trailing side, which is the unstable side or pressure side, undergoes an increase in the production of turbulence by Coriolis forces. An additional feature in rotating flows is the initiation of secondary flows in the cross-section due to rotational pressure gradients between the trailing and leading sides of the geometry. The secondary flows take the form of coherent roll cells which increase in strength with rotation number. These phenomena have been observed by many other past studies such as Johnston et al. [13], Kristoffersen and Andersson [14], Tafti and Vanka [15], Miyake and Kajishima [16], and Piomelli and Scalo [17] in turbulent channel flow, all at Reynolds numbers less than 10,000.

\subsection{Literature Review}

Patil [2] examined the effects of the first off wall LES node point of fully developed turbulent channel flow, which varied from $y^{+}$values of 15 to 70 for $R e_{\tau}$ value of 590 and 2,000 compared to the present value of $R e_{\tau}=890$. The mean streamwise velocity indicated an exact match with DNS data by Kim et al. [18] at $R e_{\tau}=590$, and wall resolved LES (WRLES) data by Piomelli [19] at $R e_{\tau}$ value of 2,000 whereas a maximum of $15 \%$ difference was observed for turbulent stresses. Also, a heat transfer study was done with WMLES with approximately 3\% difference in Nusselt number variation compared to WRLES and Dittus-Boelter correlation for fully-developed turbulent flows. WMLES without the heat transfer model indicated as much as a $25 \%$ 
underprediction of Nusselt number compared to WRLES, which indicated that the near wall heat transfer treatment was necessary for accurate results with a coarser mesh compared to the WRLES grid requirements of $y^{+}<1$.

Johnston et al. [13] took mean velocity measurements at $\mathrm{x} / \mathrm{D}=68$ where the flow was assumed to be fully developed for various rotation numbers. With an increase in rotation numbers, the mean velocity gradient, $\partial \bar{u} / \partial y$, became twice as large as the given angular velocity, $\Omega$, where absolute vorticity becomes zero (zero absolute vorticity or ZAV), which was defined as $\partial \bar{u} / \partial y=2 \Omega$. This region tended to approach closer to the pressure side with higher rotation numbers, which indicated highly turbulent flow. In addition, a series of measurements were taken over a range of bulk Reynolds number and bulk rotation numbers in order to compare the variation of wall friction velocity ratios with a reference to the stationary case. For these measurements, Preston-tube and wall slope and law of the wall methods are used independently for different Reynolds number. Further information about these methods can be found in Johnston et al. [13].

Piomelli and Scalo [17] investigated the laminarization of flows in a channel with spanwise rotation and a freestream acceleration due to a strong favorable pressure gradient. The bulk Reynolds number of 10,000 and bulk rotation number of 0.42 are simulated with DNS and LES methodologies. The grid resolution was $\Delta x^{+}=7$ and $\Delta z^{+}=$ 3.5 for DNS, $\Delta x^{+}=28$ and $\Delta z^{+}=14$ for the fine LES, and $\Delta x^{+}=42$ and $\Delta z^{+}=21$ for the coarse LES. According to Piomelli and Scalo [17], the initial zero rotation cases for all simulations were in accordance with each other. With the rotation, $R o_{b}=0.42$, the mean velocity profile was in good agreement with each other whereas the LES 
overestimated wall stresses by $30 \%$ in the coarse simulation and $25 \%$ in the fine simulation with higher percentage difference on the pressure side.

Kristoffersen and Andersson [14] performed DNS at $R e_{b}$ of 5,800 with up to 0.5 rotation number with $128 \times 128 \times 128$ grid resolution. The wall coordinates used in the simulation were $\Delta x^{+}=20, \Delta y_{1}{ }^{+}=0.5$ to 5 , and $\Delta z^{+}=10$. The overall trend was that with an increase in rotation numbers, the Reynolds stresses decreased on the leading side and increased on the trailing side due to Coriolis forces. Time averaged local wall friction velocity ratios indicated an asymptotic behavior beyond 0.15 bulk rotation number on the trailing side whereas a slight decrease in wall friction velocity ratios were observed on the leading side. At a bulk rotation number of $0.15,4$ spanwise roll cells, or TaylorGörtler vortices, were observed where every other vortex was counter-recirculating. In addition, these roll cells were more biased toward the pressure side with an increase in rotation numbers.

Similarly, Miyake and Kajishima [16] used LES with the Smagorinsky model to analyze the flow at $R e_{b}$ of 9,900 with up to 0.165 rotation number. The conclusion was that the production of Reynolds stresses and turbulent kinetic energy on the trailing side increased whereas the opposite effect was evident on the leading side. Compared to friction velocity ratio data by Johnston et al. [13], the laminarization on the leading side was not evident in the simulation. Miyake and Kajishima [16] noted that the Smagorinsky model that was used in the simulation needed to be improved in order to accurately capture the laminar or transitional fluid motion on the leading side.

DNS study was done on rotating turbulent channel flow by Lida et al. [20]. The objective of the study was to observe the behavior of friction velocity ratios on the 
leading and trailing sides with the observance of zero absolute vorticity (ZAV) in the mean velocity profile, which increased with an increase in rotation numbers. At bulk Reynolds numbers from 142 to 3,100 , rotation numbers as high as 1.0 were examined. The 3 grids that were used in simulations were $512 \times 65 \times 288$ nodes in $22 \pi \delta \times 2 \delta \times$ $10 \pi \delta$ computational domain, $64 \times 65 \times 64$ in $4 \pi \delta \times 2 \delta \times 2 \pi \delta$ computational domain, and $128 \times 129 \times 128$ in $4 \pi \delta \times 2 \delta \times 2 \pi \delta$ computational domain. As rotation numbers increase, friction velocity ratios on the leading and trailing sides indicated relatively a constant value beyond bulk Rotation number of 0.05 , and the ZAV region continued to increase. Lida et al. [20] concluded that results from 3 grid resolutions matched well with themselves and referenced experimental results.

Grundestam et al. [21] did a DNS study on rotating turbulent channel flow, which ranged from 8,052 to 21,600 in bulk Reynolds number and 0.98 to 3.00 in bulk rotation numbers, in order to examine the effects of stabilization and destabilization of the flow. For all rotation numbers, the mean velocity gradient maintained approximately $2 \Omega$. However, this $2 \Omega$-region decreased for higher rotation numbers greater than a bulk rotation number of 1.50 . Between 1.0 to 2.0 rotation numbers, the bulk velocity significantly increased due to the damping induced by Coriolis forces. In addition to the DNS and LES results from Alvelius [22], friction velocity ratios reached maximum and minimum values for the trailing and leading sides respectively at approximately 0.5 bulk rotation number. From there on, friction velocity ratios continued to decrease until the flows on both sides became laminarized due to the damping effect by Coriolis forces.

Tafti and Vanka [15] used wall resolved LES (WRLES or LES) to study turbulent statistics, roll cells, and overall flow field for bulk Reynolds number of 5,960 and 5,040 
and bulk rotation number of 0.024 and 0.143 , respectively. As the rotation number increased, large-scale spanwise roll cells increased from 2 to 4 from 0.024 to 0.143 rotation number where these roll cells were concentrated toward the trailing side as also seen in the results by Kristoffersen and Andersson [14]. In addition, wall shear stresses were analyzed on both leading trailing sides with respect to the upwash and downwash movements of spanwise roll cells. For 0.024 rotation number, upwash movement decreased the shear on the wall on both sides whereas the downwash movement indicated higher shear value on the trailing side whereas insignificant differences on the leading side were observed. The 0.143 bulk rotation number followed a similar trend as the 0.024 rotation number where the leading side did not reflect much effect of upwash and downwash movements by spanwise roll cells. However, the magnitude difference among these 2 rotation numbers in wall shear stress values increased on the trailing side whereas the leading side presented less difference in wall shear stress values. This phenomenon indicated that with an increase in rotation numbers, Coriolis forces contributed to higher shear stress values on the trailing side.

\subsection{Computational Model and Details}

The computational model assumes fully-developed and incompressible flow as mentioned in Chapter 2.3. The turbulent channel simulates a spatially repeating domain with periodic boundary conditions in the streamwise, $\mathrm{x}-$, and spanwise, $z-$, directions, where the channel consists of 2 smooth walls in the y- direction. The channel experiences a positive z-directional rotation at an angular velocity, $w_{z}$ in $\mathrm{rad} / \mathrm{s}$. The governing flow equations are non-dimensionalized by a characteristic length scale, which is $\delta$ or the 
channel half width, and a characteristic velocity scale given by the friction velocity, $u_{\tau}=\sqrt{\tau_{w} / \rho}$. The assumed periodicity of the domain in the streamwise or $\mathrm{x}$-direction requires that the mean gradients of pressure be isolated from the fluctuating periodic component as described in Chapter 2.

At a nominal bulk Reynolds number, $R e_{b}$, of 34,000 , a total of 6 cases are examined for the turbulent channel flow. Bulk rotation numbers, $R o_{b}$, of $0,0.070,0.10$, 0.21 , and 0.38 are simulated with WMLES. An additional stationary case $\left(R o_{b}=0\right)$ without WMLES was examined to validate the methodology. $R e_{b}$ and $R o_{b}$ are defined as follow:

$R e_{b}=\frac{2 \bar{u}_{b} \delta}{v}$

$R o_{b}=\frac{2 w_{z} \delta}{\bar{u}_{b}}$

where $\bar{u}_{b}$ is the time-averaged bulk velocity, $\delta$ is the channel half width, $v$ is the kinematic viscosity of the medium, which is air for all cases, and $w_{z}$ is the angular velocity in z-axis.

The computational domain for the turbulent channel flow simulations is presented in Figure 3.1. The computational domain consists of approximately $3 \times 10^{5}$ cells, or 64 $\times 50 \times 96$ cells in $i, j$, and $k$ directions where $i$ is the streamwise direction, $j$ is the wall normal direction, and $\mathrm{k}$ is the spanwise direction. A uniform grid distribution is used in each direction with spacing values of approximately $0.1,0.04$, and 0.07 in $\mathrm{i}, \mathrm{j}$, and $\mathrm{k}$ directions, respectively. These spacing values are equivalent to wall coordinates of 87,36 , and 58 in $x^{+}, y^{+}$, and $z^{+}$, respectively. Wall boundary conditions are set on both top and bottom of planes (xz-planes) whereas periodic boundary conditions are set on other planes. 
The non-dimensional time step value of $5 \times 10^{-4}$ is used and time averaging is performed for 5 non-dimensional time units. The momentum and pressure equations are solved implicitly to the convergence criteria value of $1 \times 10^{-6}$ at each time step. All turbulent channel flow cases utilize 4 cores of an Intel Xeon Linux cluster. Each time step takes approximately $10 \mu$ s of wall clock time/grid node.

In the following section, all quantities are non-dimensionalized by $u_{\tau}$ or $u_{\tau}^{2}$ unless specified differently. In addition, all referenced data, either experimental or computational, are adjusted with consistent reference values with WMLES results to ensure that comparisons are done properly. In addition, although, the $2 \Omega$-region, or neutral stability region, is widely known to be equivalent to $\partial \bar{u} / \partial y$, the presented bulk rotation number and non-dimensionalization of $y$ is based on the full channel width, and the $\bar{u}$ is normalized by the bulk mean velocity, which is equivalent to the scale on the $y-$ axis of Figure 3.5 as a reference. Therefore, the expression is expressed as $2 R o_{b}=$ $\partial\left(\bar{u} / U_{\text {mean }}\right) / \partial(y / 2 \delta)$. For the discussion, this region is referred to as the $2 \Omega$-region, instead of $2 R o_{b}$-region. All data that were used to compare to WMLES results are summarized in Table 3.1.

\subsection{Validation of Wall Modeled LES of Turbulent Channel Flow}

The validation of the near wall treatment is done without heat transfer for this geometry. In order to examine the effects of near wall treatment, the Fanning friction factor is used to approximate the inlet bulk velocity:

$c_{f_{0}}=\frac{2 \tau_{w}}{\rho \bar{u}_{b}^{2}}=0.0706 \cdot R e_{b}^{-0.25}$ 
where $\bar{u}_{b}$ is the bulk velocity, which was found to be approximately 19.1 at $R e_{\tau}$ of approximately 890 . Figure 3.2 shows the effect of the wall model on the calculated volumetric flow rate for a set value of $R e_{\tau}=890$. The case with the wall model is able to maintain a high skin friction at the walls, representative of turbulent flow, which balances the applied pressure gradient to give a bulk Reynolds number of 34,000 , whereas, the case without the wall model grossly under predicts wall friction allowing the flowrate to grow much beyond the target $R e_{b}=34,000$ as seen in Figure 3.2.

\subsection{Results of Turbulent Channel Flow with Rotation at $\operatorname{Re}=\mathbf{3 4 , 0 0 0}$}

While maintaining the bulk Reynolds number to approximately 34,000, a range of bulk rotation numbers are analyzed, which are $0,0.070,0.10,0.21$, and 0.38 . Based on the mean velocity profiles from Johnston et al. [13] for $R o_{b}$ of $0,0.068$, and 0.42 , the mean velocity profiles of WMLES are compared. As seen in Figure 3.3 for the stationary case, besides the first off-wall LES node on the trailing side with approximately $10 \%$ difference, a parabolic shape of the mean velocity profile indicates that the flow is fully developed and within $3 \%$ difference compared to the experimental data. At a bulk rotation number of 0.070 , the shift in the mean velocity profile towards the leading side is evident where a maximum of approximately $7 \%$ difference is observed as seen in Figure 3.4. With a further increase in bulk rotation number to 0.40 , WMLES results deviates from the experimental data of Johnston et al. [13] where the experimental data does not present a significant difference in $2 \Omega$-region relative to the 0.068 bulk rotation number. The experimental data appeared to be not fully developed in the central third of the channel for $R e_{b}$ of 35,000 as noted in Johnston et al. [13]. Therefore, the data by Piomelli 
and Scalo [17] were used for comparison in Figure 3.5 in spite of the difference in bulk Reynolds number. The DNS and LES mean velocity profiles for 0.42 bulk rotation number at 10,000 bulk Reynolds number by Piomelli and Scalo [17] were in good agreement with themselves and with WMLES results with a maximum of $17 \%$ difference on the leading side. The difference is primarily due to how much flow is driven by spanwise roll cells and the proximity of the roll cells with respect to the leading side, and could be partly attributed to the difference in bulk Reynolds number. DNS and LES results, specifically, mean velocity profiles, by Piomelli and Scalo [17] indicates a larger area affected by spanwise roll cells including a shift toward the leading side, which cause a higher mean local velocity near the leading side. However, WMLES result indicates that a smaller area is affected by spanwise roll cells near the leading side, which cause the maximum mean velocity to be located closer to the channel center than the DNS and LES results. All cases, DNS and LES results and WMLES results, do indicate that with an increase in rotation numbers, spanwise roll cells drive the flow, proportionally, resulting in a distinct $2 \Omega$-region.

Figure 3.6 presents mean velocity profiles for bulk rotation numbers of 0.10 and 0.21 . The $2 \Omega$-region is present in all rotation numbers except the stationary and 0.070 rotation cases. Even though there are not any mean velocity profile comparisons for 0.10 and 0.21 rotation cases, the presence of the $2 \Omega$-region and near wall velocity gradients indicate that the flow field about the central region and near the trailing side is reasonably well predicted. In general, the velocity gradient increases near the trailing wall and decreases at the leading wall with rotation. In addition, the maximum mean local velocity 
location remains relatively constant for $R o_{b}>0.2$, indicating that the flow is largely influenced by spanwise roll cells.

In order to observe the effects of Coriolis forces on spanwise roll cells, a vector field across the channel cross-section is observed as seen in Figure 3.7 where each arrow on top of each figure indicates the direction of the spanwise roll cells. Figure 3.7 (a) shows 2 spanwise roll cells across the channel, one with an elongated structure. Therefore, at a bulk rotation number of 0.070 , there are 2 distinct counter-rotating roll cells. This observation is similar to LES work by Tafti and Vanka [15] where at 0.048 rotation number, there were 2 distinct counter-rotating spanwise roll cells. Figure 3.7 (b) for $R o_{b}$ $=0.21$ shows 4 distinct roll cells, which are evenly distributed across the domain, which agrees well with the LES results of Tafti and Vanka [15] at $R o_{b}=0.28$. As the rotation number increases further to 0.38 , the roll cells gain in strength further and breakup into smaller structures (about 6 cells can be identified in Figure 3.7 (c)). As the rotation number increases, the roll cells are pushed toward the trailing side and gain in strength to transport streamwise momentum toward the leading side of the channel. This phenomenon is the reason why the mean local velocity shifts toward the leading side with an increase in rotation number, which is reflected on Figure 3.6.

In order to closely examine the turbulence contribution to the flow field and the investigation of the laminarization for the WMLES cases, Reynolds stresses were observed across the channel width where the square of global wall friction velocity, $u_{\tau}^{2}$, is used for the normalization. Among WMLES cases, as seen in Figure 3.8, the trailing side $\overline{u^{\prime} u^{\prime}}$ value was observed to increase by $16 \%$ for 0.070 and 0.10 rotation cases, after which it drops in magnitude up to $30 \%$ relative to the stationary case at the highest rotation 
number of 0.38 . Kristoffersen and Andersson [14] observed that up to 0.10 rotation number, the trailing side $\overline{u^{\prime} u^{\prime}}$ value increases, whereas a further increase in rotation numbers caused a decrease in $\overline{u^{\prime} u^{\prime}}$ value. The WMLES results are compared with the LES results of Kristoffersen and Andersson [14] at $R e_{b}=5,800$ and $R o_{b}=0.2$. It is noted that the near wall peak in $\overline{u^{\prime} u^{\prime}}$ observed in the DNS results at a $\mathrm{y}^{+}$of approximately 14-15 lies in the inner layer of the WMLES calculations and cannot be resolved by the WMLES. Outside of the modeled inner layer, the magnitudes are reasonably well predicted by the WMLES calculation at $R o_{b}=0.2$ considering the differences in Reynolds numbers. This difference in Reynolds number plays a much larger role on the leading side of the channel. Rotationally induced Coriolis forces, damp or attenuate turbulence on the leading side, leading to subsequent laminarization. In this context the Reynolds number would have a much larger impact as can be seen in the Figure. While the WMLES predicts decreasing magnitudes at the first grid node off the wall, the profiles for all rotation numbers are quite similar up to the center of the channel. The low Reynolds number DNS study however shows lower values of $\overline{u^{\prime} u^{\prime}}$ near the leading wall than the WMLES. Similar trends are seen for all the Reynolds stress terms in subsequent figures. While the flow Reynolds number is a major contributing factor to these differences, the present form of the wall model does not have the necessary near wall physics embedded in it to predict transition, which could also contribute to these differences.

Similarly, $\overline{v^{\prime} v^{\prime}}$ values of both WMLES and DNS results are in relative good agreement as seen in Figure 3.9. Unlike $\overline{u^{\prime} u^{\prime}}$ values near the wall, the trailing side $\overline{v^{\prime} v^{\prime}}$ values peak further away from the wall outside of the modeled inner layer at zero rotation. 
The peak shifts further toward the center of channel with an increase in rotation number and is resolved by the WMLES calculation. As the rotation number increases, there is a substantial increase in the level of $\overline{v^{\prime} v^{\prime}}$ which increases monotonically throughout the trailing side of the channel. The large increase in $\overline{v^{\prime} v^{\prime}}$ with rotation is attributed to the strength of the energetic secondary flow in the form of roll cells which gains strength as the rotation number increases. Comparing to the low Reynolds number results of Kristoffersen and Andersson [14], the WMLES predictions compare quite well with the DNS data on the trailing side of the channel, but over predicts at the leading side, which is a combination of the higher Reynolds number and the lack of "laminarization" physics in the wall model.

Furthermore, $\overline{w^{\prime} w^{\prime}}$ values are examined across the channel width as seen in Figure 3.10. The peak value of $\overline{w^{\prime} w^{\prime}}$ increases gradually at first and then increases more sharply on the trailing side of the channel as the rotation number increases. Comparison with DNS data shows trends similar to those found for $\overline{u^{\prime} u^{\prime}}$ and $\overline{v^{\prime} v^{\prime}}$ - good comparisons on the trailing side of the channel but over prediction on the leading side.

The turbulent shear stress, $-\overline{u^{\prime} v^{\prime}}$ is shown in Figure 3.11. These values, which directly contribute to the production of turbulence increase on the trailing side and decrease on the leading side as the rotation number increases. However, the rate of increase or decrease of the $u^{\prime} v^{\prime}$ decreases with rotation number. These also indicate that the friction velocity on the trailing side is higher than on the leading side.

The turbulent kinetic energy (TKE) variation is seen in Figure 3.12 with LES results by Tafti and Vanka [15] where the bulk rotation number is 0.286 . Higher turbulence production near the trailing side due to Coriolis forces causes higher TKE 
values near the trailing side, specifically at 0.070 rotation number. However, with a further increase in rotation numbers, peak TKE values on the trailing side decrease and stay relatively constant due to the flow dominance of spanwise roll cells (Tafti and Vanka [15]). The spanwise roll cells are responsible for transporting turbulence away from the trailing side toward the center of the channel as the rotation number increases and they gain (roll cells) in strength. On the leading side the TKE value decreases initially but remains at about the same level with increase in rotation number. Compared to the LES results of Tafti and Vanka [15] at $R o_{b}=0.286$, the predictions for $R o_{b}=0.21$ are in quite good agreement except in the region near the leading side wall, at which the low Reynolds number simulations tend to decrease the turbulent kinetic energy more than the WMLES calculations.

Johnston et al. [13] presented the wall friction velocity behavior, which is normalized by the stationary friction velocity value, of the leading and trailing sides at different bulk Reynolds numbers over a range of rotation numbers up to 0.21 with different measurement methodologies. Figure 3.13 presents all data points that were presented in Johnston et al. [13] where the dashed lines indicate the expected behavior of turbulent wall layers on both leading and trailing sides and dash-dotted line indicates the expected behavior of transitional wall layers at a Reynolds number of approximately 10,000. Figure 3.13 shows that for 10,300 and 11,400 Reynolds numbers, a laminarization on the leading side occurs, whereas higher Reynolds numbers maintain the turbulent wall layer on both leading and trailing sides over the range of rotation numbers shown. In comparison to experimental data by Johnston et al. [13] and LES results by Miyake and Kajishima [16] at 10,000 Reynolds number, WMLES results indicate 
reasonable agreement with other experimental data within $10 \%$ difference based on extrapolation. Also, as previously mentioned in Reynolds stresses and TKE discussions, Figure 3.13 indicates that there is no evidence of laminarization on the leading side. Therefore, the results present that at bulk Reynolds number of 34,000, the leading side flow does not become laminarized, which is in accordance with Johnston et al. [13] and Figure 3.13 .

\subsection{Conclusions}

The evaluation of wall modeled LES in turbulent channel flow with system rotation was done on a $64 \times 50 \times 96$ mesh at a bulk Reynolds number of 34,000. To the best of our knowledge, this is the first LES simulation of rotating channel flow at a Reynolds number beyond 10,000 . With the variation of bulk rotation numbers from 0 , $0.070,0.10,0.21$, and 0.38 , the mean velocity profiles and the development of spanwise roll cells is captured well compared to experimental and computational data. Turbulent quantities compare reasonably well on the trailing side, but did not predict the near laminarized conditions of previous DNS-LES studies at much lower Reynolds numbers. Considering the expected behavior at higher Reynolds number proposed by Johnston et al. [13], the simulation appeared to capture the attenuation of turbulence at the leading wall reasonably well. This does not rule out shortcomings in the model itself, which

presently can only react indirectly to laminarizing forces. The wall friction velocity ratios on both leading and trailing sides were calculated well within $10 \%$ difference against other data but due to a lack of comparable data at higher bulk rotation number of 0.38 at a 
bulk Reynolds number of 34,000 , the model can be used with a good degree of confidence up to bulk rotation number of approximately 0.21 . 


\subsection{Tables}

Table 3.1 A summary of referenced data used to compare present study.

\begin{tabular}{|c|c|c|c|}
\hline & Data Type & $R e_{b}$ & $R o_{b}$ \\
\hline Present & $\begin{array}{l}\text { Computational } \\
\text { (WMLES) }\end{array}$ & 34,000 & $\begin{array}{c}0,0.070,0.10,0.21 \\
0.38\end{array}$ \\
\hline $\begin{array}{c}\text { Johnston et al. } \\
\text { [13] }\end{array}$ & Experimental & $\begin{array}{c}10,300,11,400, \\
23,400,25,700, \\
33,100,34,800, \text { and } \\
36,000\end{array}$ & $\begin{array}{l}\text { A series of } 0 \text { to } 0.42 \text { at } \\
\text { different bulk Reynolds } \\
\text { number }\end{array}$ \\
\hline $\begin{array}{l}\text { Kristoffersen and } \\
\text { Andersson [14] }\end{array}$ & DNS & 5,800 & $\begin{array}{c}0,0.01,0.05,0.10 \\
0.15,0.20, \text { and } 0.50\end{array}$ \\
\hline $\begin{array}{l}\text { Piomelli and } \\
\text { Scalo [17] }\end{array}$ & DNS and LES & 10,000 & 0.42 \\
\hline $\begin{array}{c}\text { Tafti and Vanka } \\
{[15]}\end{array}$ & LES & 5,960 and 5,040 & 0.048 and 0.286 \\
\hline $\begin{array}{l}\text { Miyake and } \\
\text { Kajishima [16] }\end{array}$ & LES & 10,000 & $\begin{array}{l}\text { A series of } 0 \text { to } \\
\text { approximately } 0.165\end{array}$ \\
\hline
\end{tabular}




\subsection{Figures}

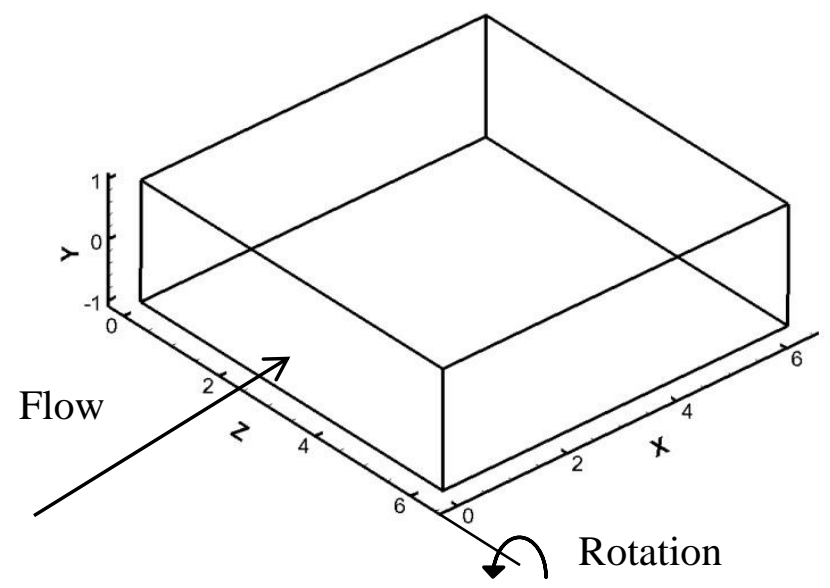

Figure 3.1 The computational domain for turbulent channel flow $(2 \pi \times 2 \times 2 \pi$ in $x$, $\mathrm{y}$, and $\mathrm{z}$ directions respectively).

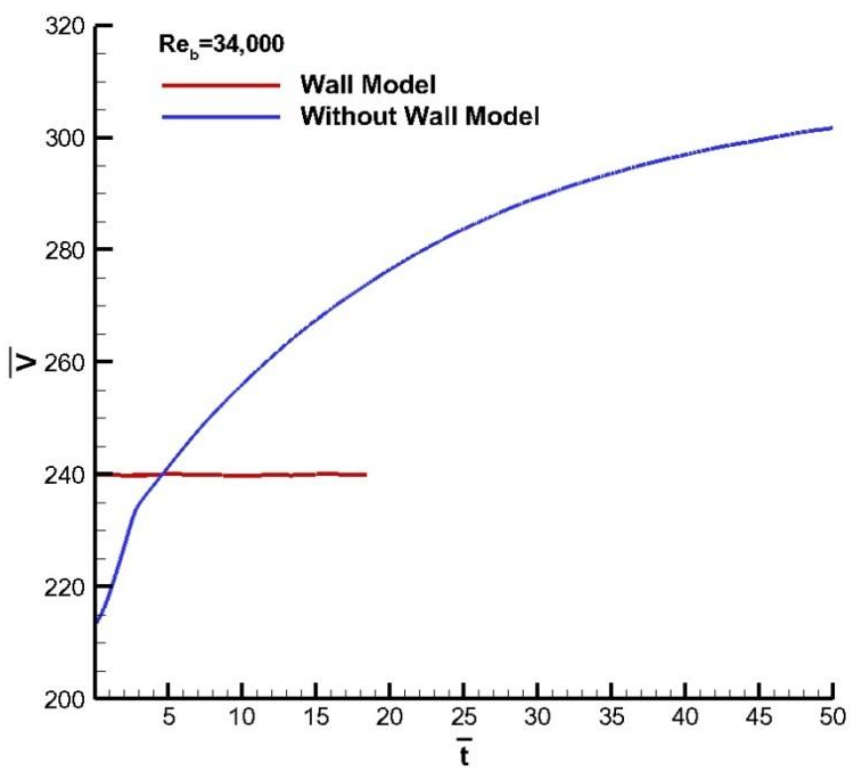

Figure 3.2 Evolution of volumetric flow rates with non-dimensional time unit for cases with the near wall treatment and without the near wall treatment. 


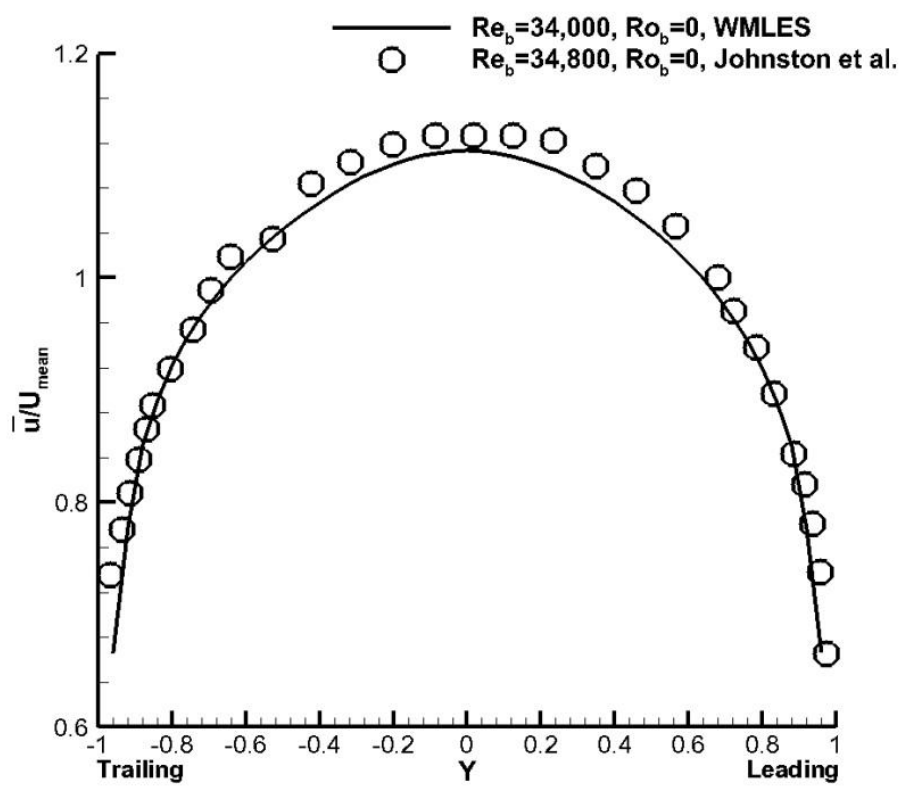

Figure 3.3 The mean velocity profile from WMLES against the experimental data by Johnston et al. [13] for Ro $=0$.

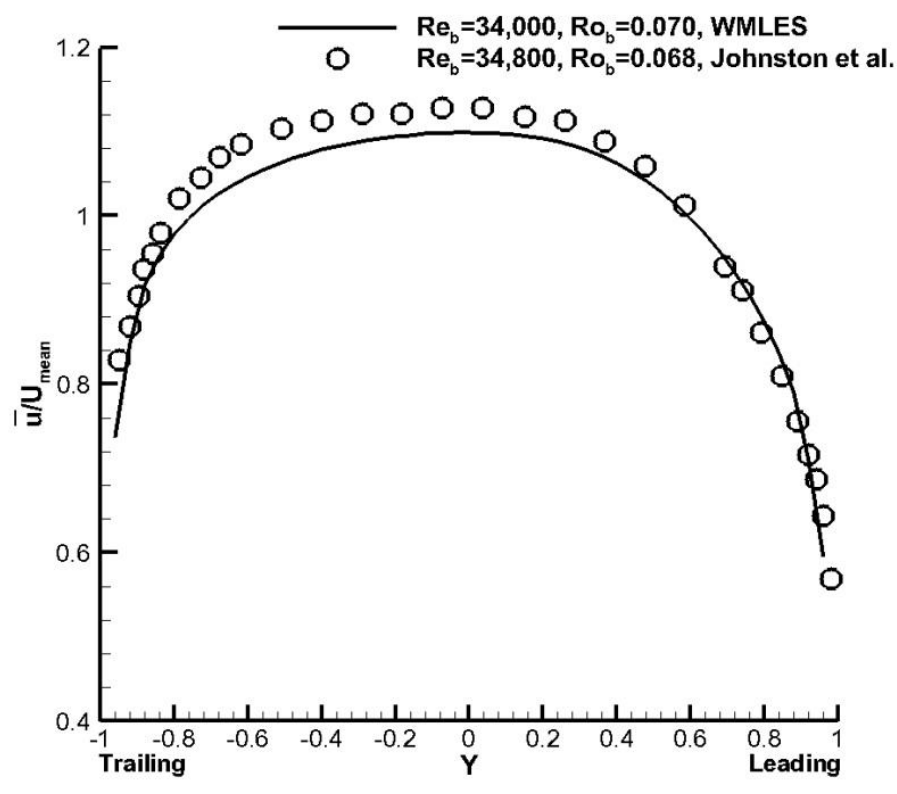

Figure 3.4 The mean velocity profile from WMLES against the experimental data by Johnston et al. [13] for approximately $R o=0.070$. 


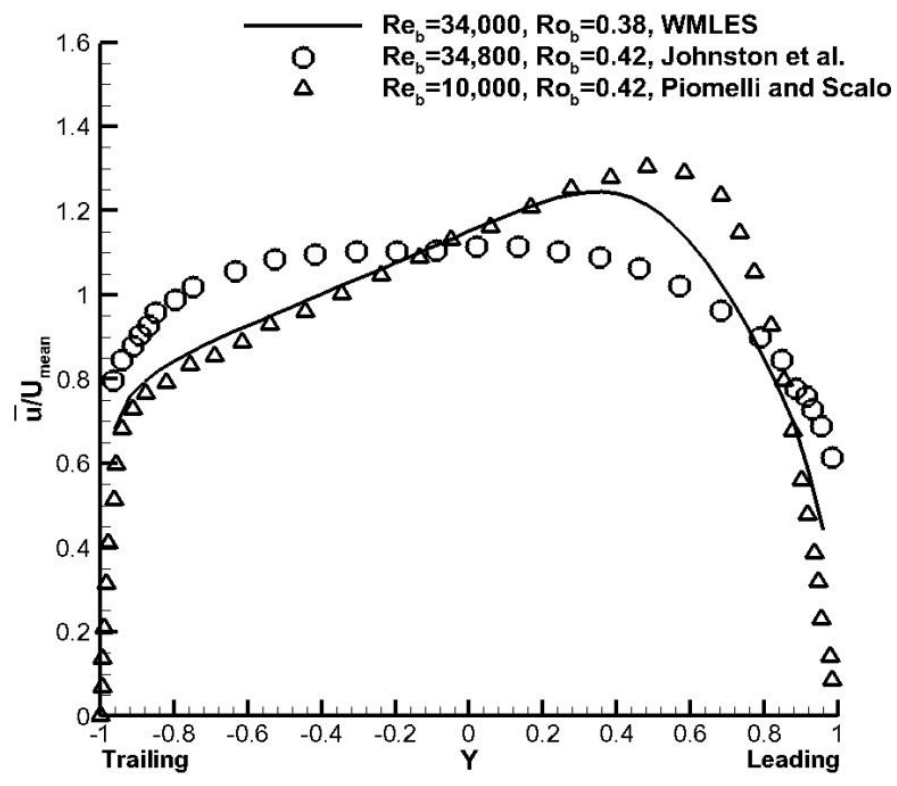

Figure 3.5 The mean velocity profile from WMLES against the experimental data by Johnston et al. [13] for approximately $\mathrm{Ro}=0.40$.

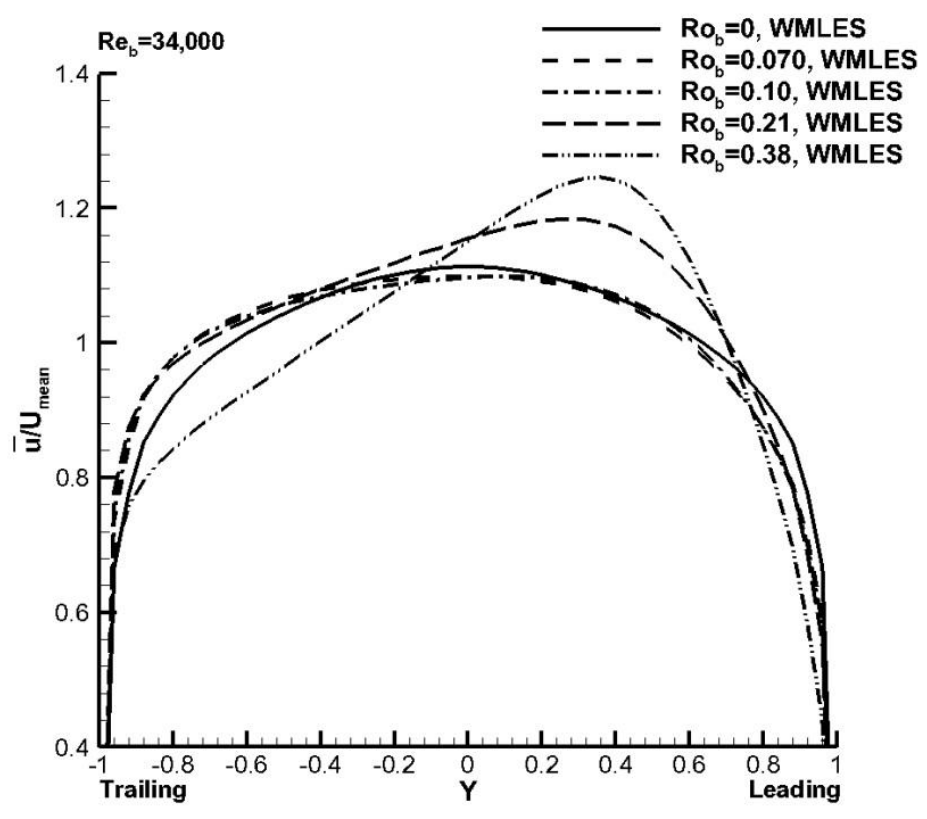

Figure 3.6 The mean velocity profiles of all rotation numbers. 

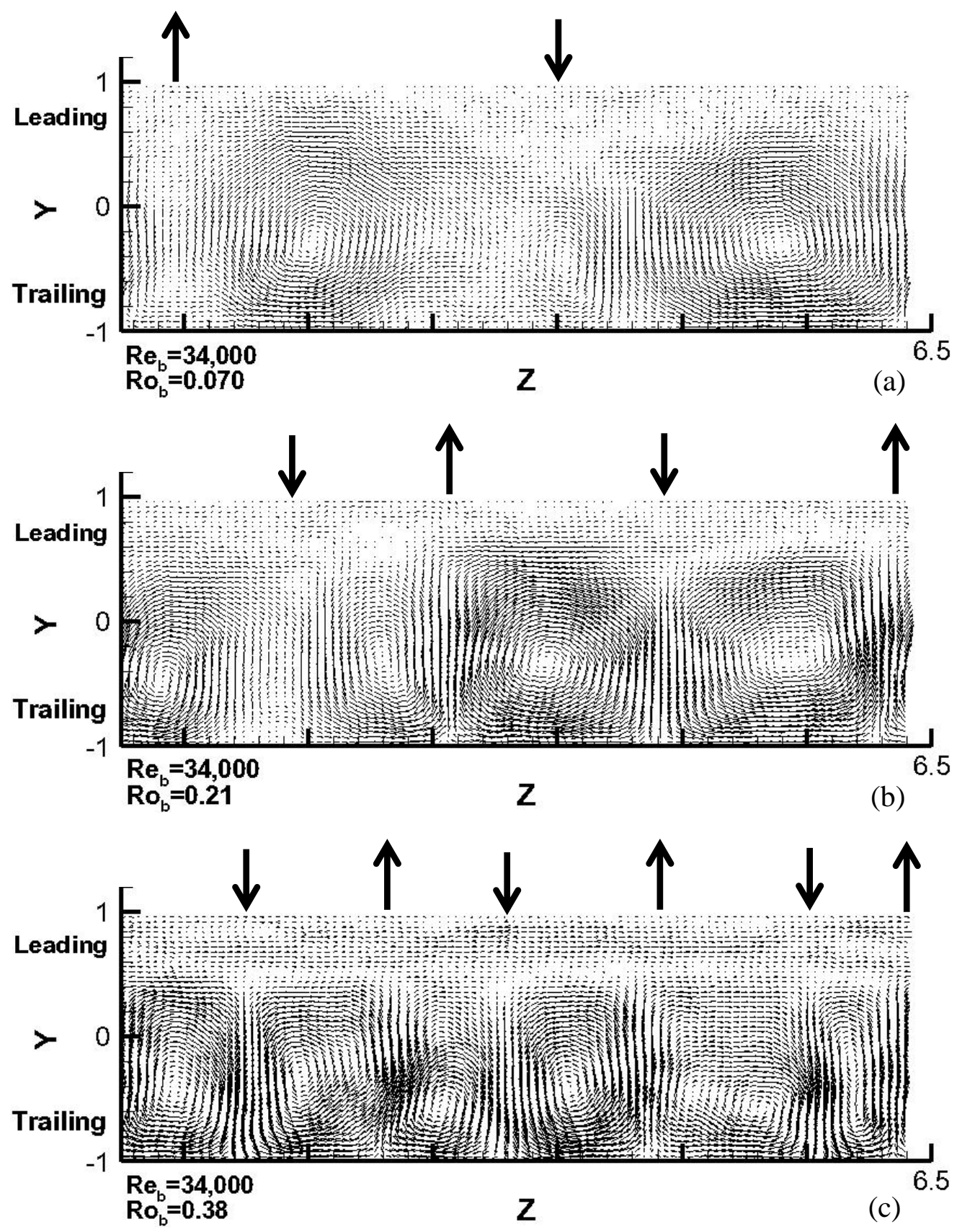

Figure 3.7 The spanwise roll cells for different rotation numbers at bulk Reynolds number at 34,000. Arrows indicate directions of the flow: (a) $R o=0.070$ (b) $R o=$ $0.21(c) R o=0.38$. 


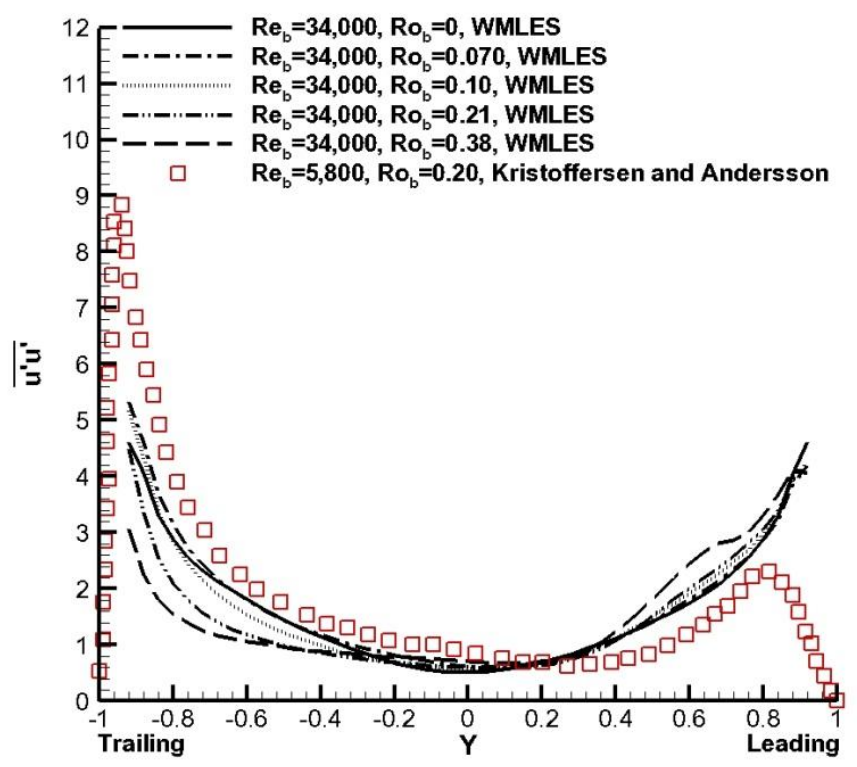

Figure $3.8 \overline{u^{\prime} u^{\prime}}$ over all rotation numbers.

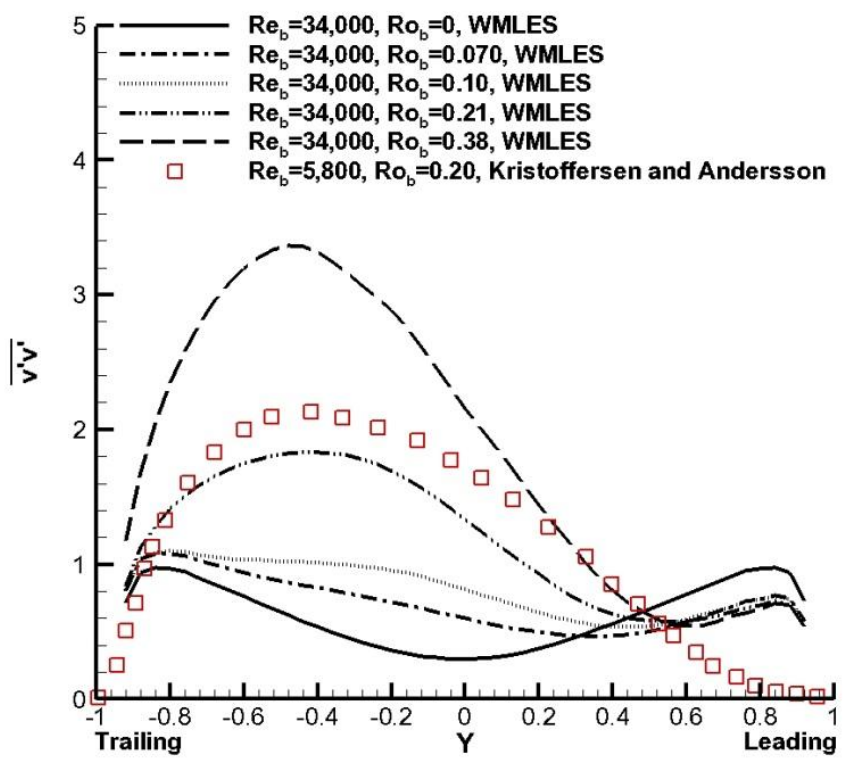

Figure $3.9 \overline{v^{\prime} v^{\prime}}$ over all rotation numbers. 


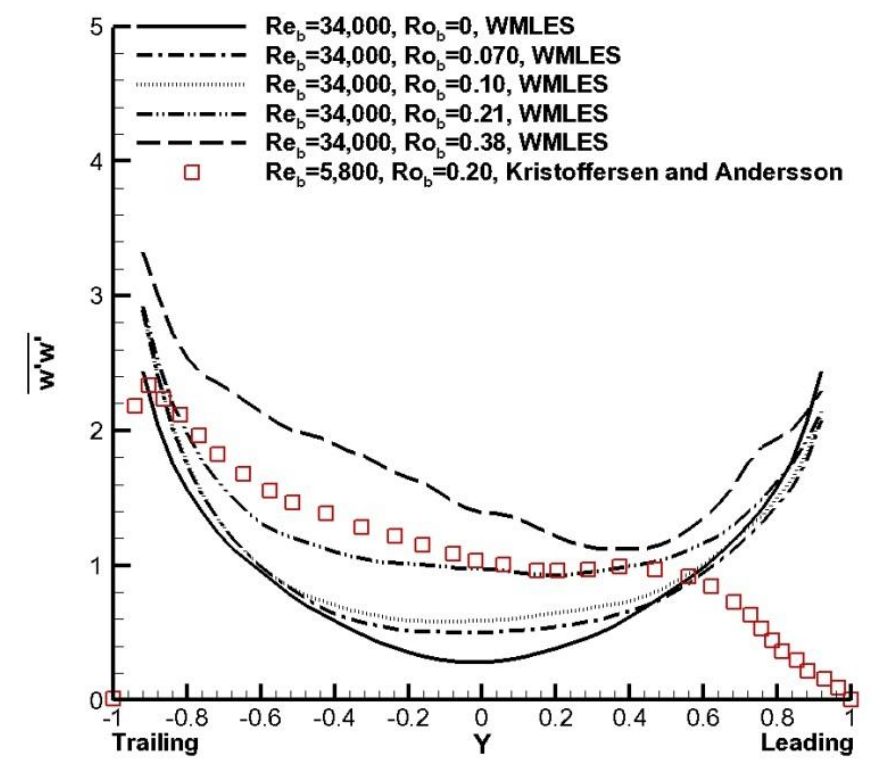

Figure 3.10 $\overline{w^{\prime} W^{\prime}}$ over all rotation numbers.

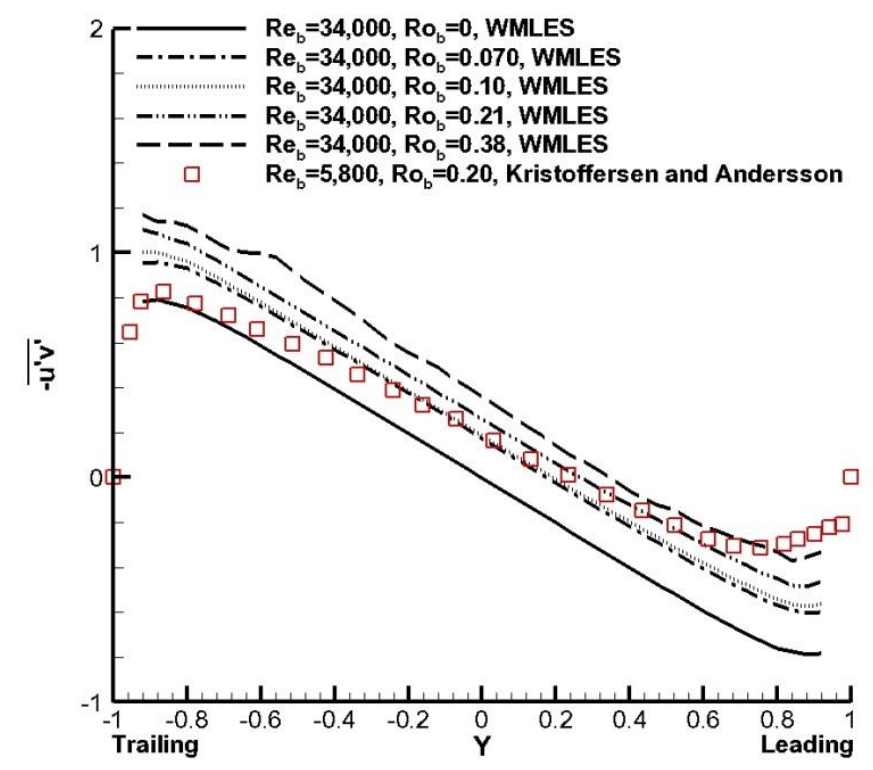

Figure $3.11-\overline{u^{\prime} v^{\prime}}$ over all rotation numbers. 


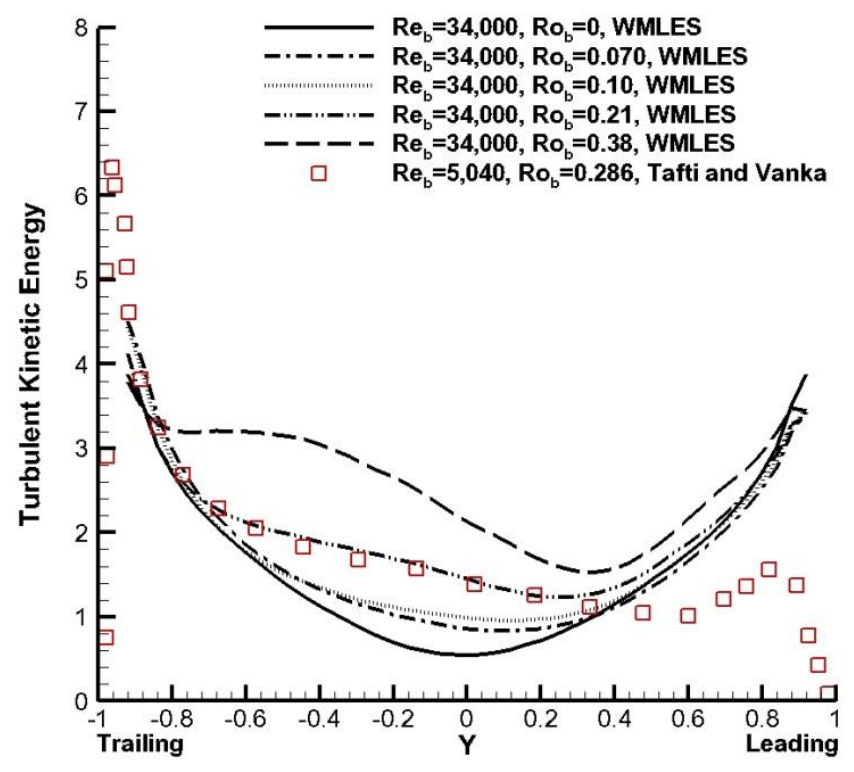

Figure 3.12 Turbulent kinetic energy of all rotation numbers. 


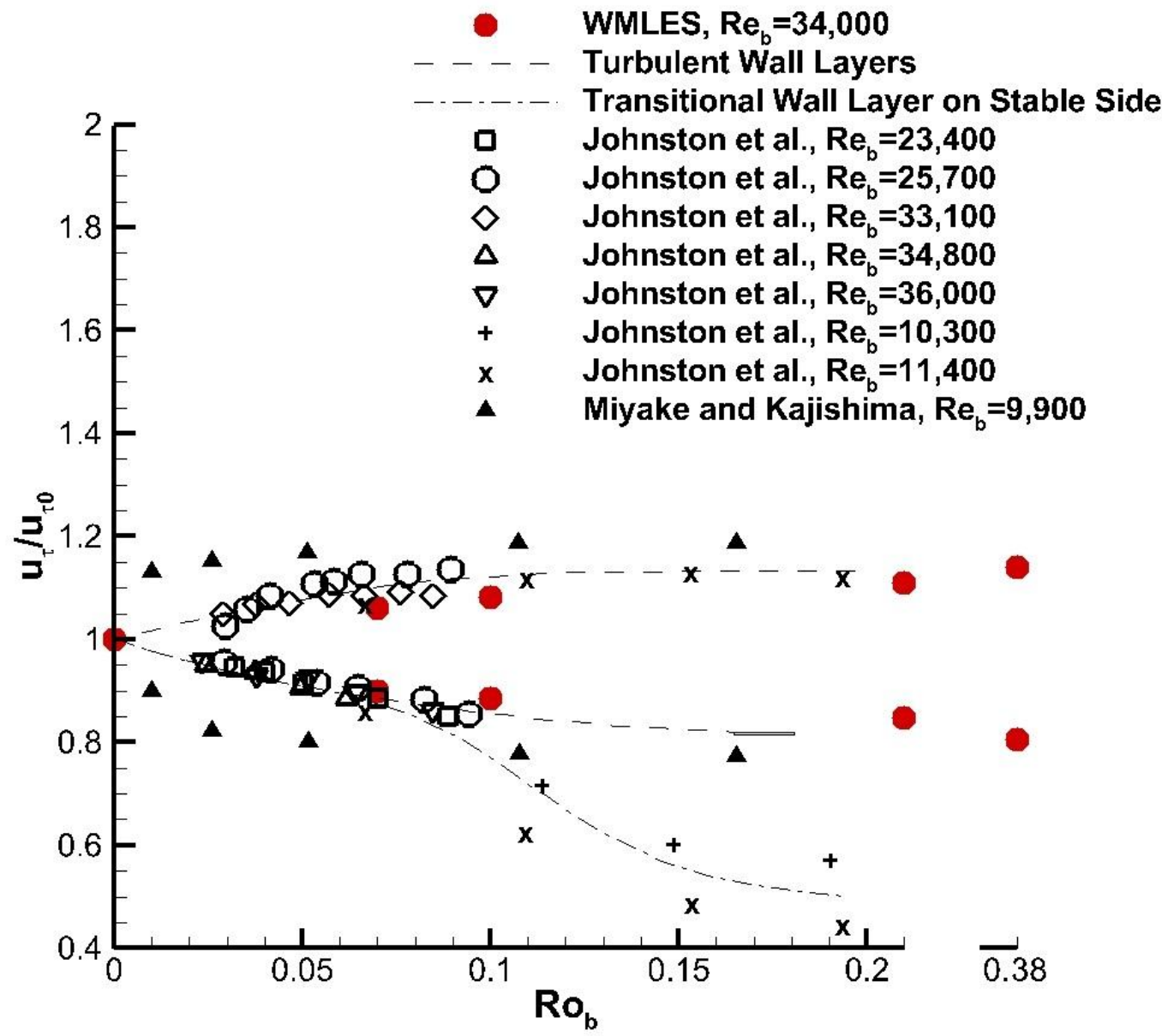

Figure 3.13 The wall friction velocity ratio plot over a range of rotation numbers is plotted where wall friction velocity is normalized by the stationary value at an equivalent bulk Reynolds number. The WMLES result is presented in $\bigcirc .---$ is the behavior expected from Johnston et al. [13] when both wall layer are turbulent. $-\cdot-\cdot-$ is the behavior when the stable side becomes transitional wall layer. Johnston et al. [13] presented results of a series of bulk Reynolds number where bulk Reynolds number of 10,300 and 11,400 data points are based on wall slope and law of the wall methods whereas other data points from different bulk Reynolds number are from Preston-tube method. Detailed methods of experimental apparatus can be found in Johnston et al. [13]. 


\section{Chapter 4 Wall Modeled Large-Eddy Simulations of $90^{\circ}$ Non- Staggered Ribbed Duct with Heat Transfer and Rotation}

\subsection{Introduction}

After evaluations of the robustness and accuracy of wall modeled LES in a turbulent channel flow, WMLES is further investigated in an orthogonal non-staggered ribbed duct with heat transfer at a bulk Reynolds number of 20,000. A rib height to hydraulic diameter ratio, $e / D_{h}$, of 0.1 and rib pitch to rib height ratio, $p / e$, of 10 were used

for the rib geometry. A stationary case was used as a baseline to observe the effects of near wall treatments. Unlike a turbulent channel flow where losses are purely frictional, the present geometry includes obstructions, which cause form drag losses. These form drag losses account for approximately $90 \%$ of losses whereas only $10 \%$ of losses are due to skin friction. The WMLES predictions are compared to the wall-resolved LES results of Tafti [23] and the experiments of Rau et al. [24].

In addition, a flow analysis with Coriolis forces and heat transfer was performed where bulk rotation numbers were varied from $0,0.09,0.17,0.36$, to 0.70 . Only the effect of Coriolis forces were included in the calculation to evaluate the wall model under the influence of these additional forces which are known to affect shear layer turbulence production on the leading and trailing sides of the duct. The WMLES results are compared to wall-resolved LES results of Abdel-Wahab [25]. 


\subsection{Literature Review}

Sewall et al. [26] evaluated a developing stationary ribbed duct case, which consisted of 3 parts: developing flow region, fully developed region, and $180^{\circ}$ bend region with $e / D_{h}$ of 0.1 and $p / e$ of 10 geometry. By using the experimental results from laser Doppler velocimetry (LDV), LES velocity profiles and turbulent statistics were compared at a bulk Reynolds number of 20,000. However, for heat transfer augmentation ratio results, additional experimental data by Fann et al. [27] were used where bulk Reynolds numbers of $10,240,20,650$, and 31,300 were evaluated with $e / D_{h}$ of 0.08 and p/e of 11 geometry at various bulk rotation numbers. Overall, the LES results showed very good agreement with the experimental data.

Ekkad and Han [28] investigated the heat transfer performance of different rib turbulator shapes and orientations of a two-pass square channel without rotation. The rib parameters were $e / D_{h}$ of 0.125 and p/e of 10 where $90^{\circ}$ parallel, $60^{\circ}$ parallel, $60^{\circ} \mathrm{V}$, and $60^{\circ}$ broken $\mathrm{V}$ ribs were tested. Bulk Reynolds numbers from 6,000, 12,000, 30,000, to 60,000 were used to observe effects on heat transfer augmentation ratios and secondary flows based on different combination of parameters. Approximately, 2 to 3 times increase in heat transfer augmentation ratios were observed due to the $180^{\circ}$ turn, which enhanced the mixing. In addition, the study showed that overall, $60^{\circ}$ broken $\mathrm{V}$ ribs increased the heat transfer in the first pass whereas $60^{\circ}$ parallel ribs increased the heat transfer within the bend and second pass.

Rau et al. [24] performed an experiment on a stationary ribbed duct with heat transfer analysis at a bulk Reynolds number of 30,000. A constant heat flux boundary condition was applied in the experiment where LDV was used to obtain flow field data. 
Air was used as a medium at a room temperature, and $e / D_{h}$ of 0.1 and $p / e$ of 6,9 , and 12 were used. However, non-staggered ribs were done only in p/e of 9 whereas only a single rib was used for other cases. The flow pattern was found to be almost symmetric about the center with a single rib geometry but an additional secondary flow was found with 2 non-staggered ribs orientation in $p / e$ of 9 . The results indicated that symmetrical ribs enhanced the heat transfer over all other configurations with a single rib. Flow impingements on top of the ribs and in front of the ribs were found in the spanwise direction, which contributed to high heat transfer augmentation ratios on smooth walls.

Tafti [23] studied quasi-DNS and LES methods on $96^{3}$ and $128^{3}$ grid resolutions at bulk Reynolds number of 20,000. Experimental data by Rau et al. [24] were used to compare results from LES with and without the dynamic Smagorinsky model (DSM). A $10 \%$ difference was seen with DSM compared to $20 \%$ difference without DSM. The computational domain was used where a $\Delta y^{+}$value was below 1 with 4 to 5 mesh points within 10 wall units to resolve the near-wall turbulence. Also, wall coordinate values between 5 to 30 were used in streamwise and spanwise directions, respectively. Tafti [23] presented that overall heat transfer augmentation ratios and friction coefficient values were underpredicted slightly compared to the experimental results by Rau et al. [24]. However, the major flow structures, turbulent parameters, and secondary flows were captured accurately by the LES to within experimental uncertainty.

Abdel-Wahab [25] evaluated effects of Coriolis forces by using LES. Both $96^{3}$ and $128^{3}$ grid resolutions were used with $e / D_{h}$ of 0.1 and $p / e$ of 10 at a bulk Reynolds number of 20,000. While using LES results of a stationary ribbed duct case by Tafti [23], as previously mentioned, bulk rotation numbers of $0.18,0.35$, and 0.67 were evaluated 
with heat transfer. Overall, turbulent statistics and heat transfer results indicated an increase in these variables on the trailing side due to Coriolis forces whereas the leading side showed an opposite effect. In addition, heat transfer augmentation ratios resulted in relatively asymptotic behavior beyond a bulk rotation number of 0.18 within $3.7 \pm 5 \%$ on the trailing side whereas a consistent decrease in heat transfer augmentation ratios was observed on the leading side. The friction factor was found to peak between bulk rotation numbers of $0.18-0.35$, after which there was a gradual decrease.

Comparing to the experimental data by Rau et al. [24], a validation of WMLES was done by Patil [2]. While maintaining the grid resolution at $56 \times 56 \times 48$ with a $\Delta y^{+}$ range from 20 to 30 at a bulk Reynolds number of 20,000 , the near wall treatment deviated by up to $10 \%$ in the frictional coefficient and within $8 \%$ difference in heat transfer augmentation ratio values compared to experimental data by Rau et al. [24]. In addition, a higher grid resolution of $72 \times 72 \times 64$ with a $\Delta y^{+}$range from 15 to 30 at an identical bulk Reynolds number of 20,000 indicated a similar result within $10 \%$ difference. In contrast, without the near wall treatment in $56 \times 56 \times 48$ geometry, heat transfer augmentation ratio values showed as much as $28 \%$ difference with $24 \%$ difference in the Fanning friction factor.

Viswanathan and Tafti [29] investigated the effects of Coriolis forced and centrifugal buoyancy in ribbed duct geometry by using detached eddy simulations (DES). A bulk Reynolds number of 20,000 was used with bulk rotation numbers from 0.18 to 0.67 and effective buoyancy parameters, $B o$, up to 0.29 where $B o=\left(\Delta \rho / \rho_{0}\right)\left(r / D_{h}\right) R o_{b}^{2}$. With an increase in buoyancy parameters at a bulk rotation number of 0.35 , heat transfer augmentation ratios on the trailing wall increased from 4.5 to 6.0 near the reattachment 
region whereas the leading wall slightly decreased. Similar to observations made by Abdel-Wahab [25], both leading and trailing sides showed asymptotic behaviors of heat transfer augmentation ratio values for different buoyancy parameters where the results were within $7.5 \%$ of LES results by Abdel-Wahab [25].

Wagner et al. [30] presented a study on a two-pass orthogonal and circular ribbed duct with a U-bend. At bulk Reynolds number of 25,000 with $e / D_{h}$ of 0.1 and p/e of 10 , different rotations were applied to study flow behavior with centrifugal buoyancy. Different combinations of bulk rotation numbers of $0,0.12,0.23$, and 0.35 and density ratios of $0.07,0.13,0.18$, and 0.22 were examined. With an increase in rotation numbers at a fixed density ratio of 0.13 , heat transfer ratio increased as much as approximately $40 \%$ on the trailing side compared to $46 \%$ decrease on the leading side within the first pass relative to the stationary case. In contrast, the trailing side second pass heat transfer ratio decreased by approximately $47 \%$ whereas the leading side stayed relatively the same within $15 \%$. In addition, the leading side heat transfer ratio increased by $10 \%$ in the second pass compared to the first pass. For the purpose of the study, which is a fullydeveloped orthogonal ribbed duct with rotation, the measurements at $x / D_{h}=8$ are used in order to avoid the entrance effect before the bend.

Parsons et al. [31] did an experimental study on rotating two-pass square channel with $90^{\circ}$ rib turbulators. Bulk Reynolds and rotation numbers were varied from 2,500 to 25,000 and 0 to 0.352 , respectively. The rib parameters were $e / D_{h}$ of 0.125 and $p / e$ of 10 where buoyancy effects were included in the study with different wall heating conditions, which were constant temperature, constant heat flux, and a hotter trailing wall than leading wall without any heat inputs on side walls (insulated). While holding a bulk 
rotation number at 0.088 , bulk Reynolds numbers were varied from 5,000 to 10,000 to observe effects of Coriolis forces. The results indicated that heat transfer augmentation ratios showed minimal difference in both first and second passes of the channel. In addition, compared to smooth wall results by Han et al. [32] with identical flow parameters without rib turbulators, heat transfer augmentation ratios increased approximately 2 to 3 times with rib turbulators.

\subsection{Computational Model and Details}

The computational model uses the fully-developed model mentioned in Chapter 2.3. The ribbed duct experiences a positive z-direction rotation at an angular velocity, $w_{z}$ in $\mathrm{rad} / \mathrm{s}$. The governing flow equations are non-dimensionalized by a characteristic length scale, which is a hydraulic diameter, $D_{h}$, and a characteristic velocity scale given by the

friction velocity, $u_{\tau}=\sqrt{\Delta \bar{P}_{x} / \rho}$. The assumed periodicity of the domain in the streamwise or $\mathrm{x}$-direction requires for the pressure and temperature to be decomposed into an inlet value as mentioned in Chapter 2.

At a nominal bulk Reynolds number, $R e_{b}$, of 20,000 , a total of 4 cases are examined for the ribbed duct flow. Bulk rotation numbers, $R o_{b}$, of $0,0.09,0.17$, and 0.36 are simulated with WMLES where an additional stationary case without WMLES was examined to validate the methodology. $R e_{b}$ and $R o_{b}$ are defined as follow:

$$
\begin{aligned}
& R e_{b}=\frac{\bar{u}_{b} D_{h}}{v} \\
& R o_{b}=\frac{w_{z} D_{h}}{\bar{u}_{b}}
\end{aligned}
$$


where $\bar{u}_{b}$ is the time-averaged bulk velocity, $D_{h}$ is a hydraulic diameter, $v$ is the kinematic viscosity of the medium, which is air for all cases, and $w_{z}$ is the angular velocity about the z-axis.

The computational domain for the ribbed duct simulations is presented in Figure 4.1. The computational domain consists of approximately $4 \times 10^{5}$ cells, or $80 \times 80 \times 64$ cells in $\mathrm{i}, \mathrm{j}$, and $\mathrm{k}$ directions where $\mathrm{i}$ is the streamwise direction, $\mathrm{j}$ is the wall-normal direction, and $\mathrm{k}$ is the spanwise direction. A non-uniform grid distribution is used in $\mathrm{x}$ and y directions with spacing values of approximately 0.006 and 0.006 , respectively, which are equivalent to $\Delta x_{1}{ }^{+}$and $\Delta y_{1}{ }^{+}$values of 21. A uniform grid distribution is used in $\mathrm{z}$ direction with a spacing value of 0.016 or a $\Delta z^{+}$value of 52 . These values were based on the specified $R e_{\tau}$, which is approximately 6,667 on average. The $e / D_{h}$ of 0.1 and p/e of 10 are used for all simulations as previously mentioned. Wall boundary conditions are set on both $\mathrm{xy}$ - and xz-planes whereas periodic boundary conditions are set on yzboundary planes.

The non-dimensional time step value of $1 \times 10^{-4}$ is used and time averaged for 5 non-dimensional time units. The momentum and pressure equations are solved implicitly to the convergence criteria value of $1 \times 10^{-6}$ at each time step. All ribbed duct cases utilize 4 cores of an Intel Xeon Linux cluster. Each time step takes approximately $6.1 \mu$ s of wall clock time/grid node.

For the heat transfer analysis, Nusselt number is used where the local Nusselt number is defined as:

$$
N u=\frac{1}{\theta_{s}-\theta_{\text {ref }}}
$$


where $\theta_{s}$ is the surface temperature and $\theta_{\text {ref }}$ is the reference temperature with the use of a constant heat flux boundary condition, or Neumann boundary condition. The reference temperature is calculated based on the averaged temperature field across the geometry:

$\theta_{\text {ref }}=\frac{\iint\left|u_{1}\right| \theta d A_{x}}{\iint\left|u_{1}\right| d A_{x}}$

Then, the averaged Nusselt number is calculated based on the local Nusselt number:

$\overline{N u}=\frac{1}{\iint_{\Omega} d S}\left[\iint_{\Omega} \frac{1}{\theta_{s}-\theta_{\text {ref }}} d S\right]$

where $S$ is the appropriate surface for the averaged Nusselt number. In addition, the averaged Nusselt number is normalized by the Dittus-Boelter equation:

$N u_{0}=0.023 \cdot R e_{b}^{0.8} \cdot \operatorname{Pr}^{0.4}$

The Fanning friction factor that is used for the ribbed duct analysis is defined as follows:

$f=\frac{1}{2 \cdot \bar{u}_{b}{ }^{2}}$

All quantities are non-dimensionalized by $u_{b}$ or $u_{b}^{2}$ unless specified. Also, Reynolds number and rotation numbers are equivalent to bulk Reynolds number and bulk rotation number, $R e_{b}$ and $R o_{b}$, respectively. Stationary LES results are from Tafti [23] and LES results with rotation are from Abdel-Wahab [25].

\subsection{Validation of Wall Modeled LES of $90^{\circ}$ Non-Staggered Ribbed Duct}

A validation of $90^{\circ}$ non-staggered ribbed duct was done based on experimental results by Rau et al. [24] and LES results by Tafti [23]. While Patil [2] observed a significant underprediction of mean Nusselt augmentation ratios and the Fanning friction factor by as much as $28 \%$ without the near wall treatment for a coarser geometry, $56 \times$ 
$56 \times 48$, the current study indicates that the presented geometry does not show a significant difference with and without the wall model. While in turbulent channel flow, the wall heat transfer is mostly dependent on near-wall turbulence production, in a ribbed duct, it is not as dependent on turbulence production in the vicinity of the wall. The turbulent characteristics of a ribbed duct are mostly driven by the turbulence production in the shear layers which form on the ribs, and which transport turbulence to near the walls and augment heat transfer. Hence, overall heat transfer results are not as dependent on the near wall model as in the case of canonical flows such as turbulent channel flow, in which most of the turbulence production occurs in the near wall layer. This is manifested in the high form losses in a ribbed duct which typically account for $90 \%$ of the pressure losses.

The heat transfer and friction coefficient results from these calculations are summarized in Table 4.1. The WMLES results are marginally better than without the wall model, but no large differences as observed by Patil [2] are evident. The ribbed wall Nusselt augmentation ratios with wall model showed $4 \%$ overprediction with respect to LES, whereas approximately $10 \%$ overprediction was observed without the wall model. Even though both cases showed an underprediction of approximately $20 \%$ for the rib augmentation ratio values, the averaged smooth wall augmentation ratio value with wall model showed $7 \%$ underprediction compared to $13 \%$ overprediction without wall model. Consequently, the overall Nusselt augmentation ratios with ribs and the Fanning friction coefficient values were reasonable well predicted within 5\%. The reattachment length as seen in Figure 4.7 is approximately 4.5 times the rib height compared to 4.2 times the rib height with wall model in Patil [2] and 4.0 to 4.25 times the rib height by Rau et al. [24]. 
Similar to the analysis that was done in the previous chapter with turbulent channel flow calculations, volumetric flow rates are given in Figure 4.2. The near constant value of the volumetric flow rate establishes that the flow has attained a steady state. In addition, the time evolution of the spatially averaged Nusselt number on different surfaces of the ribbed duct is plotted in Figure 4.3 for calculations with and without the wall model. In all cases the Nusselt numbers remain stationary over the period of time-averaging.

Figure 4.4 shows the streamwise velocity distributions at $y / e=0.1$ against Rau et al. [24], which are normalized to fit against $p / e=10$ due to different pitch ratios. The streamwise velocity decreases significantly shortly after the trailing edge of the rib in the recirculation region. Upon flow reattachment, the streamwise velocity again till it encounters the leading edge of the rib. The negative velocities are a result of the mean junction eddy which forms at the leading edge. Figure 4.5 shows a superimposed plot of the wall-normal velocity distribution at $y / e=0.1$ against Rau et al. [24], which are also normalized to fit against $p / e=10$ due to different pitch ratios. The wall-normal velocity steadily decreases until the reattachment location, and then increases. Compared to the experimental data points, WMLES predicts both velocity distributions reasonably well.

In addition, turbulent profiles of WMLES are compared against LES results by Tafti [23], which are normalized by $u_{b}$ for rms values and $u_{b}^{2}$ for $-\overline{u^{\prime} v^{\prime}}$ as shown in Figure 4.6. These profiles were extracted at the end of the computational domain $(x / e=$ $4.5)$ at center plane $(z=0.5)$. All turbulent profiles are in very good agreement with the wall-resolved LES results. The peak turbulent intensities are observed in the separated 
shear layer, which form on the ribs, indicating that the presence of the rib is instrumental in the production of turbulence.

\subsection{Results of $90^{\circ}$ Ribbed Duct with Rotation at $\operatorname{Re}=20,000$}

While maintaining a bulk Reynolds number at approximately 20,000, different bulk rotation numbers with WMLES were analyzed against LES results by Abdel-Wahab [25]. Coriolis forces stabilize and destabilize the flow on the leading and trailing sides of

the duct, respectively, and consequently affect the region of separation behind the rib, which is a primary recirculation zone. Based on previous studies, there are four distinct recirculation zones present; a small mean junction eddy at the front of the rib and on top of the rib, a small counter-recirculation zone behind the rib, and a primary recirculation zone behind the rib. As seen in Figure 4.7, for the stationary ribbed duct case, both leading and trailing sides indicate the same flow field about the center where the reattachment length is approximately $x / e=4.5$. The primary difference is that the eddy on top of the rib is not captured on either side of the geometry. This region is also absent in other rotation cases except 0.70 rotation case on the trailing side as seen in Figure 4.8, which appears to be due to effects of grid resolution. With an increase in bulk rotation number, for $R o_{b}$ of 0.70 , the primary recirculation zone reattachment length on the trailing side reduced by $11 \%$ relative to the stationary case with approximately $x / e=4.5$. In contrast, for $R o_{b}$ of 0.36 , the primary recirculation zone reattachment length on the leading side is overpredicted by $33 \%$ compared to LES results as seen in Figure 4.9. However, the trailing side reattachment length was reasonable within $13 \%$ difference against LES data. In addition, the flow field about the center of the ribbed duct of 
WMLES results indicated that the flow is biased toward the trailing side whereas the opposite effect is seen in LES results with Coriolis forces. Considering the behavior of the reduction in primary recirculation zone reattachment length with an increase in rotation numbers, the trailing biased flow field is expected. While Abdel-Wahab [25] presented that the primary recirculation zone on the leading side increases by $56 \%$ at $R o_{b}$ of 0.67 , the presented study indicates that the reattachment length increased by $33 \%$, which was relatively constant compared to 0.36 bulk rotation number in WMLES case.

The mean wall-normal (v) and spanwise (w) velocities in different halves of $R o_{b}$ of 0.36 are seen in Figure 4.10 to observe the secondary fluid motion, which drove the flow in turbulent channel case at higher bulk rotation numbers. The wall-normal velocity indicates a strong upwash movement near the smooth wall, which does not reach all the way up to the leading side rib wall. As the flow moves away from the smooth wall, a downwash movement is seen near the center of the geometry. The spanwise velocity on the trailing side indicates a strong impingement of the flow on the smooth wall near the rib on the trailing side, resulting in high Nusselt augmentation ratio values, which is seen in Figure 4.21. In addition, the wall-normal and spanwise velocity contours indicate that the secondary flow, or spanwise roll cells, moves closer to the trailing side with an increase in rotation numbers where only two primary spanwise roll cells are evident from these contours. The shift in spanwise roll cells was also observed in turbulent channel flow in the previous chapter, which indicates that the secondary flow behavior is relatively similar at higher rotation numbers.

The turbulent Reynolds stress values reflect the effect of Coriolis forces as well. As bulk rotation number increases, the trailing side turbulent stresses increase whereas 
the leading side shows an opposite effect. Similarly, the effects on leading side shear layer decrease as the bulk rotation number increases. For further analysis of how Reynolds stresses vary at a high bulk rotation number, contour plots of Reynolds stresses at 0.36 bulk rotation number is observed in Figure 4.11 and Figure 4.12 where each plot is normalized by $\bar{u}_{b}$ for $u_{r m s}, v_{r m s}$, and $w_{r m s}$ and $\bar{u}_{b}^{2}$ for $\overline{u^{\prime} v^{\prime}}$. The streamwise turbulent fluctuation, $u_{r m s}$, in Figure 4.11 (a) indicates that in the separated shear layer on top of the trailing side rib, the $u_{r m s}$ values increase as high as 2.4 times compared to the stationary case while an insignificant difference is found on the leading side at a bulk rotation number of 0.36. Comparing WMLES to LES, the $u_{r m s}$ distribution in the trailing side separated shear layer indicates that the WMLES over-predicts the strength of the shear layer, which based on these values seems to maintain its strength further downstream than that indicated by the LES. Conversely, WMLES seems to underpredict $u_{r m s}$ levels in the immediate wake of the rib.

The wall-normal fluctuation, $v_{r m s}$, in Figure 4.11 (c) indicates maximum values in the separated shear layer as high as 2.1 times the stationary case on the trailing side. As shown in Figure 4.11 (d) of LES results, the wall-normal fluctuations peak near the front edge of ribs on the trailing side, which is also evident in WMLES results in Figure 4.11 (c). Both WMLES and LES predict this quantity in reasonable agreement with two notable differences. WMLES underpredicts $v_{r m s}$ in the immediate wake of the ribs, especially on the leading side. Additionally, higher values of $v_{r m s}$ are predicted by the LES in the trailing half of the duct. A similar discrepancy is also seen in $u_{r m s}$ and $w_{r m s}$ distributions. As rotation number increases, the secondary flow in the cross-section grows in strength and is responsible for turbulent transport from the trailing wall to the center of 
the duct. From these results it can be surmised that a weaker secondary flow is predicted by WMLES.

The spanwise turbulent fluctuation, $w_{r m s}$, exhibit peak values at the front of the rib, which is the region of highly unsteady junction eddies. These eddies are also responsible for the high heat transfer augmentation in this region on the ribbed walls. These unsteady eddies also exist in stationary ducts and increase in strength on the trailing side and decrease in strength on the leading side as the rotation number increases. The predictions with WMLES and LES are in good agreement except for some of the differences mentioned earlier. It is noted that the turbulence is largely anisotropic in the shear layers and in the vicinity of the ribbed walls.

Comparisons of the turbulent shear stress, $\overline{u^{\prime} v^{\prime}}$, are shown in Figure 4.12 (c) and (d). The shear stress magnitudes are highest in the separated shear layers on the ribs as expected and the WMLES predictions are in good agreement with LES with some minor differences. As with the values of $u_{r m s}$, high values of turbulent shear stress persist longer in the WMLES than LES.

Turbulent kinetic energy (TKE) contours are seen in Figure 4.13 and Figure 4.14 for all bulk rotation number cases. The effect of Coriolis forces on the TKE plot is not as evident as individual Reynolds stresses as shown in the figures. While the leading side TKE values decreases near the shear layer on top of the rib, TKE values of the shear layer on the trailing side increases with an increase in rotation numbers up to $R o_{b}$ of 0.36 , after which it decreases at the higher rotation number at 0.70 . Similar to the discussion in turbulent channel flow, with an increase in bulk rotation numbers, the secondary flow appears to dominate the flow. This phenomenon leads to relatively steady TKE values at 
$R o_{b}$ of 0.70 on the trailing side while maintaining the low TKE region behind the rib. In addition, compared to the stationary case, the size of this region behind the rib diminishes up to $40 \%$ at $R o_{b}$ of 0.36 where a small counter-recirculation zone resides, which is evident in LES results as well. On the leading side, the size of the region behind the rib increases compared to the stationary case whereas the shear layer TKE continues to diminish as the bulk rotation number increases. In comparison to LES results, as seen in Figure 4.14 (b), (d), and (f), contours of WMLES show relatively similar trends and magnitudes where the most difference is seen for a bulk rotation number of 0.17 .

For further analysis of TKE, a comparison plot of WMLES and LES results are shown in Figure 4.15 in the center of the geometry $(x=1.0$ and $z=0.5)$. Compared to LES results in Figure 4.15 (b), WMLES results indicate a slight overprediction by up to $8 \%$ for the stationary case and up to $15 \%$ for a 0.36 bulk rotation case. With an increase in rotation numbers, the leading side TKE stays relatively constant within $11 \%$ difference at most, which is also observed in LES results as seen in Figure 4.15 (b). With higher rotation numbers at $0.09,0.17$ and 0.36 , the maximum value of TKE values on the trailing side increased by 6,18 , and $27 \%$ relative to the stationary case, respectively. However, at $R o_{b}$ of 0.70 , the TKE value decreased by $22 \%$ compared to $13 \%$ decrease in LES results. Overall, the TKE distributions are in relatively good agreement between the LES and WMLES with some overprediction of the maximum value at the trailing side.

Heat transfer analysis is done on the leading and trailing sides to observe the effects of Coriolis forces and secondary flow motion. For the stationary case, as seen in Figure 4.16 (a), the recirculation zone behind the rib has the lowest mean heat transfer. In contrast, the region in front of the rib indicates the highest local mean heat transfer 
augmentation ratio due to an unsteady junction eddies, which is in accordance with Reynolds stress analysis and the LES result shown in Figure 4.16 (b). As seen in Figure 4.16 and Figure 4.17, the mean heat transfer augmentation ratio values on the leading side in front of the rib decreases with an increase in rotation numbers. In addition, the low heat transfer augmentation ratio region behind the rib continues to increase further downstream with decrease in heat transfer augmentation ratios, which is due to an increase in the size of the recirculation zones behind the rib as seen in Figure 4.8, and is also evident in LES results in Figure 4.16 (c) and Figure 4.17. The WMLES tends to overpredict the extent of these regions compared to LES. The opposite effect is seen on the trailing side in Figure 4.16 (d) and Figure 4.18. As the bulk rotation number increases on the trailing side, the low heat transfer augmentation ratio region behind the rib decreases consistently. In contrast, the extent of high heat transfer augmentation increases in size and magnitude in the reattachment region which is due to the direct effect of Coriolis forces and also due to the effect of the secondary flow. The shifting of the zone of maximum heat transfer in LES from the spanwise center of the duct to offcenter at $R o_{b}=0.35$ is a consequence of secondary flow impingement on the trailing wall. This shift is not captured well by WMLES. While the zone of high heat transfer in WMLES spreads in the spanwise direction toward the smooth walls, the maximum heat transfer mostly occurs at the center. Another difference between LES and WMLES is the high heat transfer augmentation at $R o_{b}=0.70$ compared to the LES results. The WMLES results, as seen in Figure 4.18 (e), indicate that the shear layer from the rib is stronger and persists further downstream, which result in as much as $23 \%$ difference compared to the LES result in Figure 4.18 (f). This could also be due the fact that the WMLES calculation 
does not capture the secondary flow to its full extent, which tends to disrupt the shear layer and transport it away from the trailing wall.

Mean streamlines and mean heat transfer augmentation ratios along the trailing side centerline are superimposed in Figure 4.19. The high heat transfer augmentation ratio in front of the rib is a result of unsteady junction eddies which form in this region and is manifested as a mean recirculation zone. The heat transfer augmentation ratio is low in the primary recirculation zone behind the rib but this recirculation zone reaches a maximum value in the reattachment region. There appears to be overprediction of heat transfer augmentation ratio by as much as $33 \%$ downstream of reattachment. As previously mentioned, the overprediction of heat transfer augmentation ratio appears to be due to an overprediction of the strength of the shear layer.

Heat transfer augmentation ratios of smooth walls were observed in Figure 4.20 and Figure 4.21. Unlike leading and trailing heat transfer augmentation ratio values, the values on smooth walls are underpredicted by WMLES. While the impingement due to secondary flow and spanwise velocity, as seen in Figure 4.10, cause a high heat transfer augmentation ratio near the edge of the trailing side rib, the difference is as much as $35 \%$ compared to LES results by Abdel-Wahab [25] for 0.35 rotation case (Figure 4.21 (c) and (d)). However, the diminishing shear layer region on the leading side is evident with an increase in rotation numbers whereas the region on the trailing side increases. The strong upwash movement near the trailing side at $R o_{b}$ of 0.36 , as seen in Figure 4.10 , is reflected in the figure where $52 \%$ of the maximum heat transfer augmentation ratio is maintained near the vicinity of the shear layer compared to $65 \%$ of LES results by AbdelWahab [25]. 
Heat transfer augmentation ratios on ribs are in accordance with the expectation that the values are the highest near the leading edge of the rib on both leading and trailing sides whereas augmentation values are the lowest behind the ribs. Unlike LES results by Abdel-Wahab [25] where a larger part of the windward face of the rib on the trailing side shows high heat transfer augmentation ratios, WMLES results indicate a much smaller region. In addition, LES results indicate that there is a small region near the front edge of the top surface on the trailing rib where heat transfer augmentation ratio value is low, which increases downstream as shown in Figure 4.23 (b). The opposite effect is seen in WMLES results where the front edge of the top surface on the trailing rib indicates high heat transfer augmentation ratios, which decreases downstream (Figure 4.23 (a)). This is due to the fact that the coarser grid resolution used in WMLES is unable to capture the small recirculation zone which forms in this region.

Furthermore, comparisons of various heat transfer augmentation ratio and losses against LES results by Tafti [23] and by Abdel-Wahab [25] are seen in Table 4.2. While most of the heat transfer augmentation ratio results show that WMLES is in agreement with LES results, significant underpredictions of heat transfer augmentation ratio on the ribs are observed as seen in Figure 4.22 and Figure 4.23. Overall heat transfer augmentation ratios with ribs show $11 \%$ underprediction compared to $28 \%$ underprediction for ribs alone. Both the leading and trailing side augmentation ratio values are predicted within $10 \%$ by WMLES but the difference in augmentation ratio on the leading side increases with increase in rotation.

In addition to heat transfer augmentation ratio values, form drag losses and the Fanning friction factor are analyzed. Form and friction losses indicated relatively good 
comparisons with LES results to within $10 \%$ difference whereas for $R o_{b}$ of 0.17 and 0.36 , the frictional losses remain relatively constant to within 5\%. Also, Coriolis forces do not appear to significantly affect either form drag or frictional losses as seen in Table 4.2.

Heat transfer augmentation ratio variations of leading and trailing sides are examined for all bulk rotation numbers as seen in Figure 4.24. In conjunction with LES results by Abdel-Wahab [25], experimental data by Wagner et al. [30] are also used to compare the augmentation variations. While bulk Reynolds number and geometry were identical to Abdel-Wahab [25], Wagner et al. [30] included the effects of buoyancy with a density ratio at 25,000 bulk Reynolds number, which are different from the present study. Also, the rib arrangement was staggered with circular ribs with $e / D_{\mathrm{h}}=0.1$ and $p / e=10$. However, the fully developed region data at $x / D_{h}=8$ were extracted for the comparison with the density ratio of $0.13(\Delta \rho / \rho=0.13)$. For the experimental data, oneto-one match with the present study is hard to justify. However, the data nevertheless gives some indication of experimental values, which are valuable. At $R o_{b}$ of 0.09 , the leading and trailing side heat transfer augmentation ratios are overpredicted by approximately $14 \%$ on average whereas at $R o_{b}$ of 0.17 , both sides are approximately the same against LES results. At $R o_{b}$ of 0.36 , WMLES tends to overpredict the heat transfer augmentation ratio on the trailing and leading sides. For 0.70 bulk rotation number, the leading and trailing side augmentation values differ by approximately $16 \%$, which are reasonable. Considering the dominant secondary flow presence at high bulk rotation numbers in these chapters, a bulk rotation number of 0.70 is expected to stay relatively constant compared to the value of 0.36 rotation number. In spite of these differences, WMLES compares favorably with LES results within $16 \%$ difference. 


\subsection{Conclusions}

The wall model was able to capture the gross effects of Coriolis forces in a ribbed duct. The flow destabilization on the trailing side and stabilization on the leading side of the duct were identified through analysis of recirculation zones, turbulent stresses and heat transfer augmentation ratios. The WMLES tended to overpredict the recirculation region on the leading side compared to LES. Overall, the turbulent stresses were predicted with good accuracy. From indirect evidence, it was surmised that the secondary flow was not resolved as well as it should have. This affected the value and region where maximum heat transfer occurred on the trailing wall as the rotation number increased, where the secondary flow supposedly gets stronger and starts influencing the flow dynamics in the duct. In addition, heat transfer augmentation caused by direct impingement on the windward face of the rib was grossly underpredicted. It is not clear whether this is a result of deficiencies in the wall model or the coarser grid resolution which was used. Overall, trailing and leading wall heat transfer augmentations were predicted to within $16 \%$ of LES, while reducing the cost of simulations by a factor of 10 20. 


\subsection{Tables}

Table 4.1 The summary of ribbed duct calculation with and without wall treatment against wall resolved LES by Tafti [23] and experimental data by Rau et al. [24].

\begin{tabular}{|c|c|c|c|c|}
\hline & $\begin{array}{l}\text { With Wall } \\
\text { Treatment }\end{array}$ & $\begin{array}{c}\text { Without Wall } \\
\text { Treatment }\end{array}$ & $\begin{array}{c}\text { WRLES } \\
\text { (Tafti [23]) }\end{array}$ & $\begin{array}{c}\text { Experiment } \\
\text { (Rau et al. [24]) }\end{array}$ \\
\hline $\begin{array}{c}\text { Mesh Size } \\
(\mathrm{x}, \mathrm{y}, \mathrm{z})\end{array}$ & $80 \times 80 \times 64$ & $80 \times 80 \times 64$ & $128 \times 128 \times 128$ & Not applicable \\
\hline$R e_{\tau}$ & 6,662 & 6,664 & 6,667 & Not applicable \\
\hline$R e_{b}$ & 19,999 & 19,775 & 20,000 & 30,000 \\
\hline $\begin{array}{c}\text { Form Drag } \\
\text { Loss }(\%)\end{array}$ & 90 & 91 & 91 & 85 \\
\hline $\begin{array}{l}\text { Reattachment } \\
\text { Length }\left(x_{r} / e\right)\end{array}$ & 4.5 & 4.3 & 4.1 & 4.0 to 4.25 \\
\hline \multicolumn{5}{|c|}{$\overline{N u} / N u_{0} \quad\left(N u_{0}=0.023 \cdot \operatorname{Re}_{b}^{0.8} \cdot \operatorname{Pr}^{0.4}\right)$} \\
\hline Leading & 2.50 & 2.68 & 2.40 & 2.40 \\
\hline Trailing & 2.50 & 2.68 & 2.40 & 2.40 \\
\hline Ribs & 2.32 & 2.34 & 2.89 & Not applicable \\
\hline Smooth & 1.75 & 1.65 & 1.89 & 2.05 \\
\hline $\begin{array}{l}\text { Overall with } \\
\text { Ribs }\end{array}$ & 2.14 & 2.17 & 2.23 & Not applicable \\
\hline $\begin{array}{c}\text { Overall } \\
\text { without Ribs }\end{array}$ & 2.11 & 2.14 & 2.14 & 2.21 \\
\hline \multicolumn{5}{|c|}{$f / f_{0} \quad\left(f_{0}=0.046 \cdot R e_{b}^{-0.2}\right)$} \\
\hline Overall & 8.74 & 8.93 & 8.6 & 9.5 \\
\hline
\end{tabular}


Table 4.2 The summary of ribbed duct calculation of all rotation cases. LES results

by Tafti [23] are used for the stationary case and LES results by Abdel-Wahab [25] for non-zero rotation cases.

\begin{tabular}{|c|c|c|c|c|c|c|c|c|c|}
\hline & WMLES & LES & WMLES & WMLES & LES & WMLES & LES & WMLES & LES \\
\hline Mesh & $80 \times 80 \times 64$ & $128^{3}$ & $80 \times 80 \times 64$ & $80 \times 80 \times 64$ & $128^{3}$ & $80 \times 80 \times 64$ & $128^{3}$ & $80 \times 80 \times 64$ & $128^{3}$ \\
\hline$R e_{\tau}$ & 6,662 & 6,667 & 6,662 & 7,003 & 6,660 & 7,003 & 6,660 & 7,003 & 6,660 \\
\hline $\mathrm{Re}_{\mathrm{b}}$ & 19,999 & 20,000 & 19,182 & 20,478 & 18,821 & 19,172 & 18,981 & 20,047 & 19,980 \\
\hline$R o_{\tau}$ & 0 & 0 & 0.27 & 0.5 & 0.5 & 1.0 & 1.0 & 2.0 & 2.0 \\
\hline $\mathrm{Ro}_{\mathrm{b}}$ & 0 & 0 & 0.09 & 0.17 & 0.18 & 0.36 & 0.35 & 0.70 & 0.67 \\
\hline $\begin{array}{l}\text { \% Form } \\
\text { Losses }\end{array}$ & 90 & 91 & 91 & 89 & 93 & 89 & 91 & 87 & 87 \\
\hline \multicolumn{10}{|c|}{$N u_{\text {avg }} / N u_{0}\left(N u_{0}=0.023 \cdot R e_{b}^{0.8} \cdot \operatorname{Pr}^{0.4}\right)$} \\
\hline Ribs & 2.32 & 2.89 & 2.46 & 2.42 & 3.38 & 2.48 & 2.97 & 2.45 & 2.78 \\
\hline Leading & 2.50 & 2.4 & 1.89 & 1.64 & 1.71 & 1.63 & 1.36 & 1.43 & 1.18 \\
\hline Trailing & 2.50 & 2.4 & 3.46 & 3.62 & 3.7 & 3.94 & 3.63 & 4.41 & 3.76 \\
\hline Smooth & 1.75 & 1.89 & 1.96 & 2.02 & 2.32 & 2.14 & 2.32 & 2.35 & 2.45 \\
\hline Overall & 2.14 & 2.23 & 2.32 & 2.33 & 2.62 & 2.45 & 2.48 & 2.60 & 2.50 \\
\hline \multicolumn{10}{|c|}{$f / f_{0} \quad\left(f_{0}=0.046 \cdot R e_{b}^{-0.2}\right)$} \\
\hline Overall & 8.7 & 8.6 & 9.4 & 9.3 & 9.8 & 10.4 & 9.6 & 9.62 & 8.75 \\
\hline
\end{tabular}




\subsection{Figures}

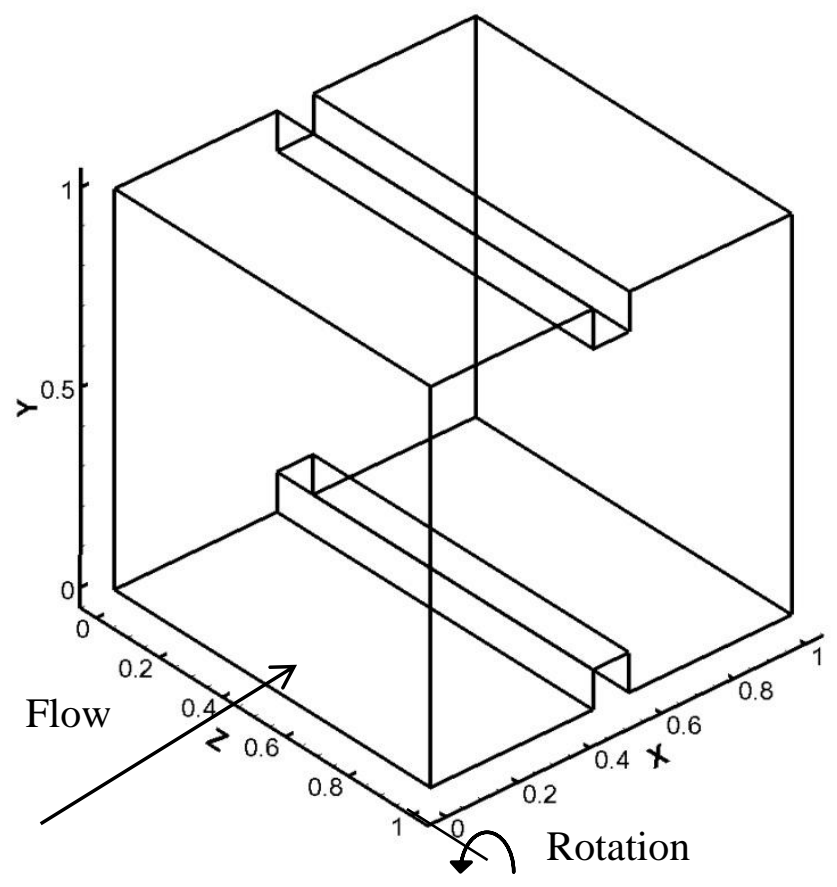

Figure 4.1 The computational domain for ribbed duct.

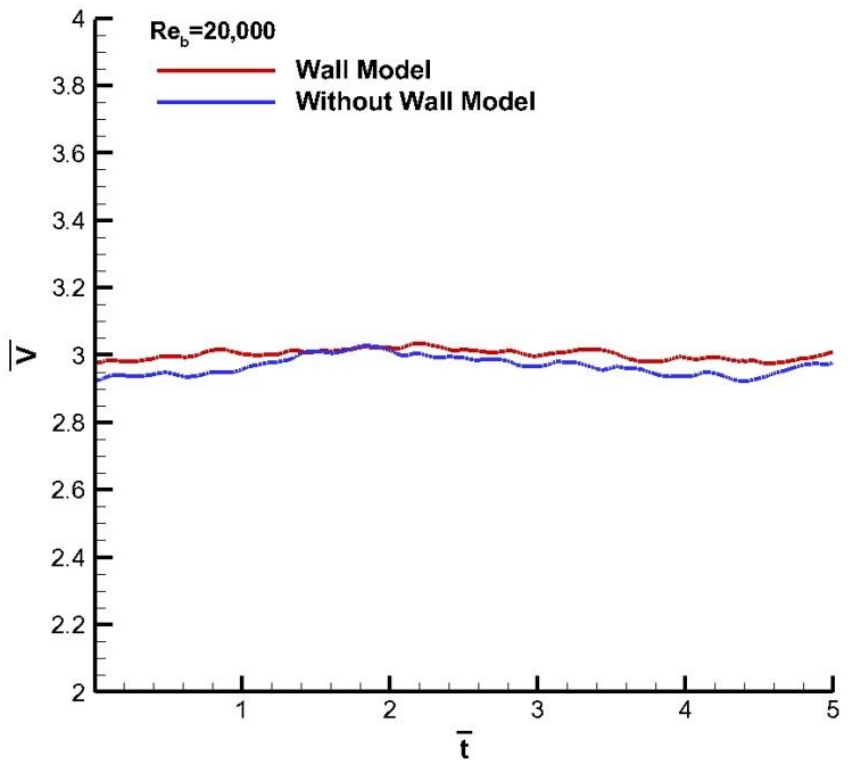

Figure 4.2 Volumetric flow rates over a non-dimensional time unit for cases with the near wall treatment and without the near wall treatment. 


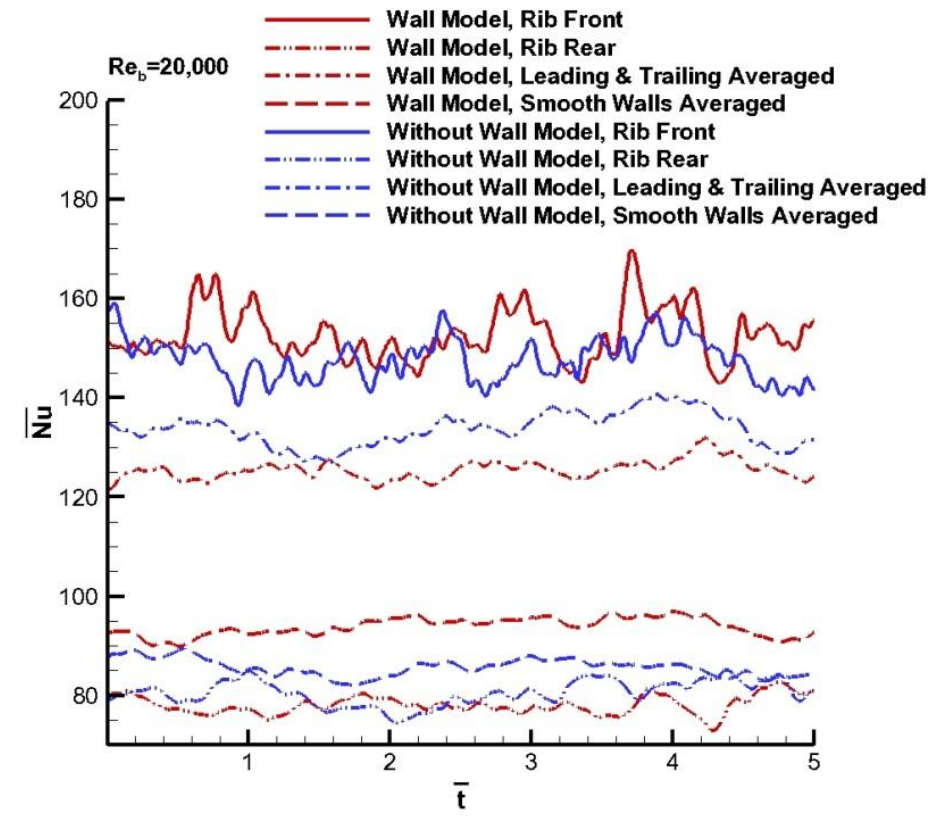

Figure 4.3 Mean Nusselt values over a non-dimensional time unit for cases with the near wall treatment and without the near wall treatment for different walls. Leading and trailing walls are averaged along with both smooth walls, separately.

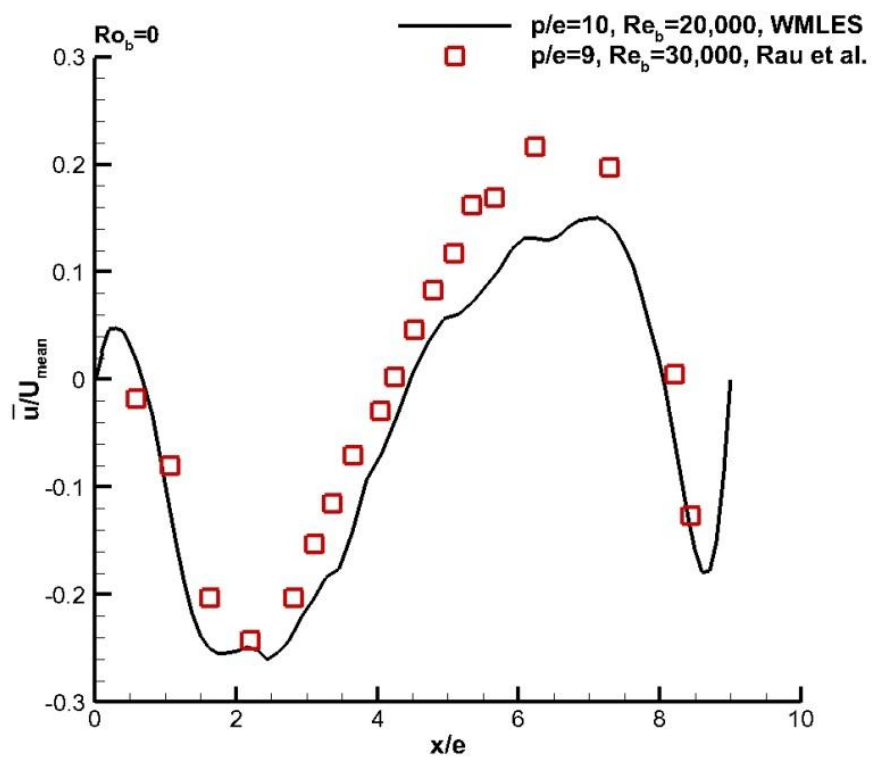

Figure 4.4 Mean streamwise velocity distribution along streamwise direction at $y / e=$ 0.1 and $z=0.5$. 


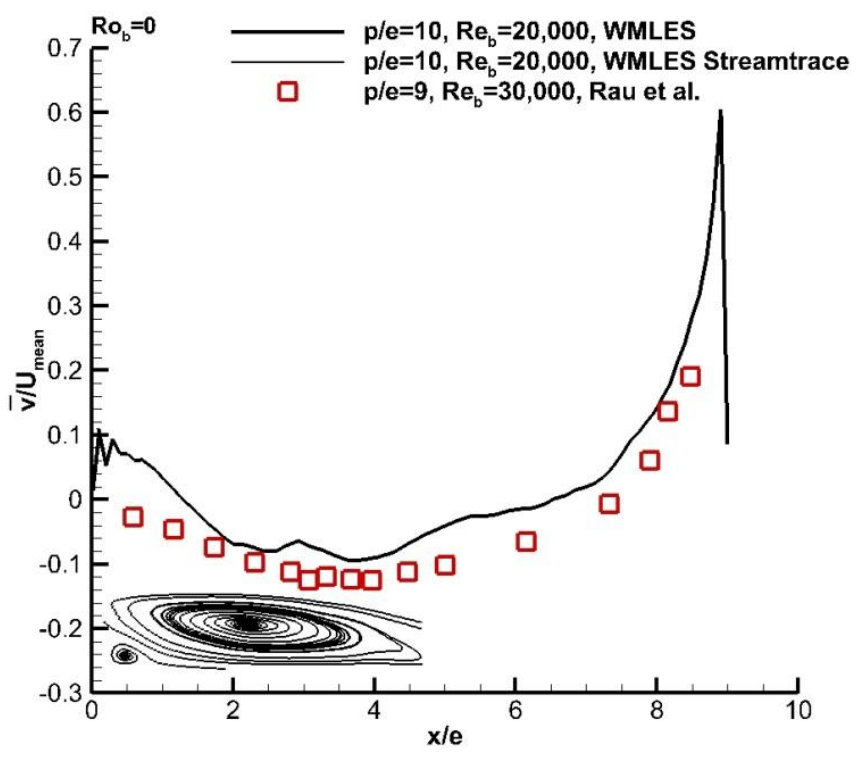

Figure 4.5 Mean wall-normal velocity distribution along streamwise direction at $y / e$ $=0.1$ and $z=0.5$.

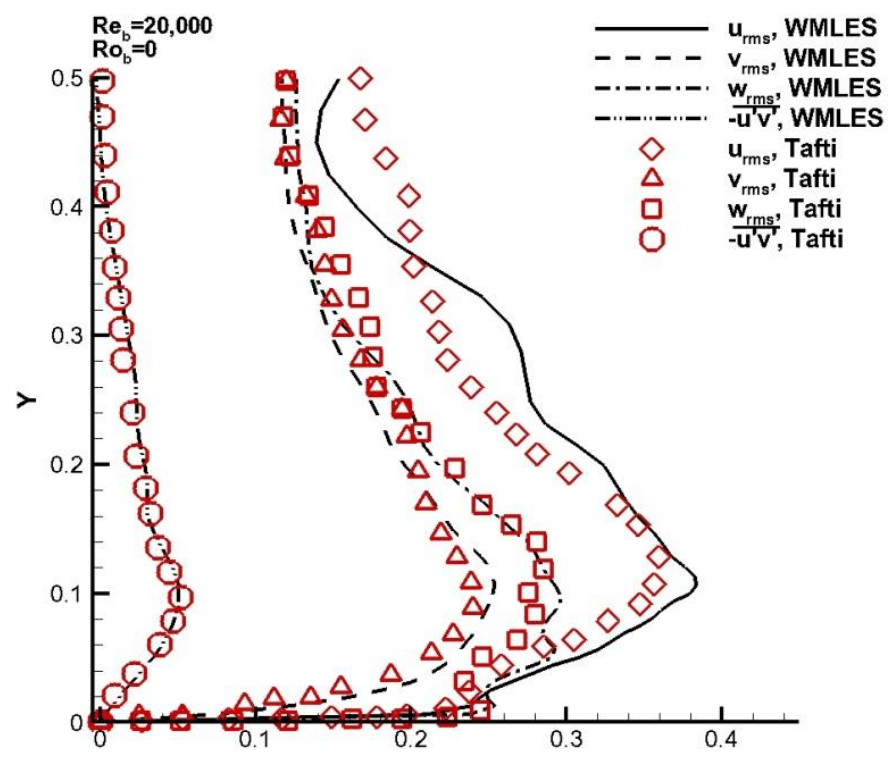

Figure 4.6 Turbulent profiles at center plane $(z=0.5)$ for a bottom half of the domain at $x / e=4.5$ downstream of the rib. 


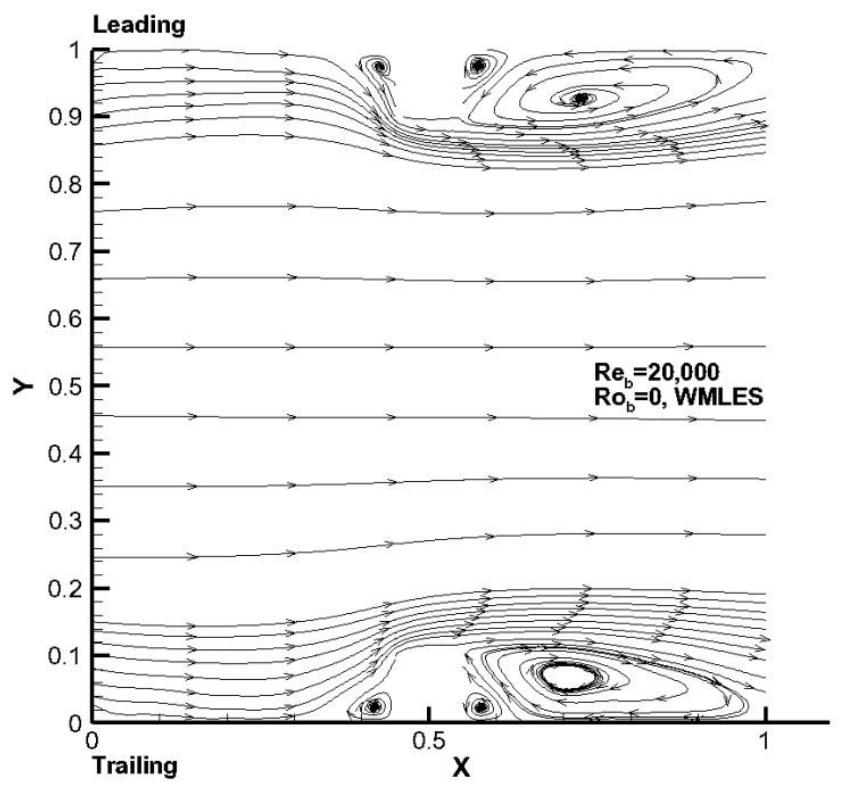

Figure 4.7 Mean flow streamlines of the stationary ribbed duct case. 


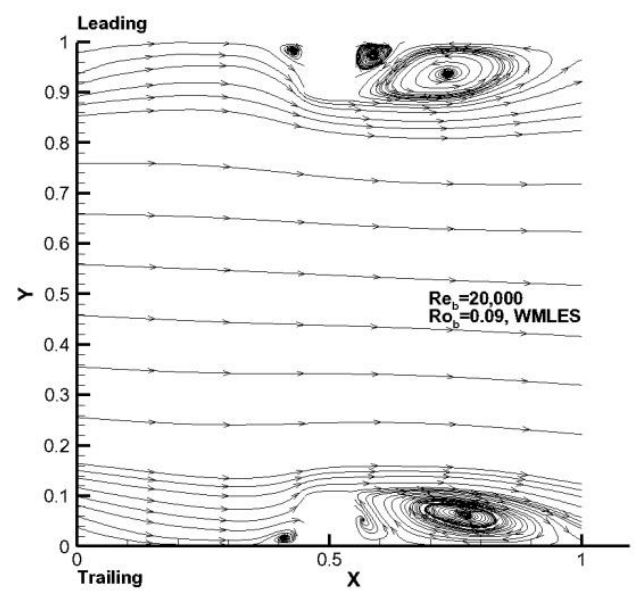

(a)

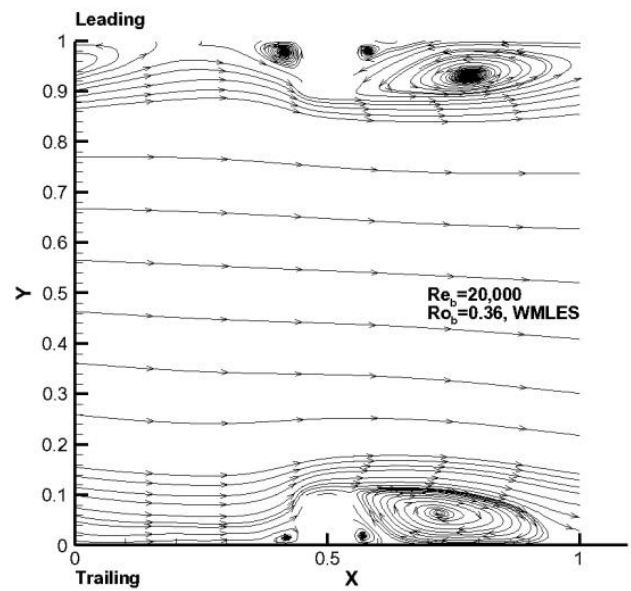

(c)

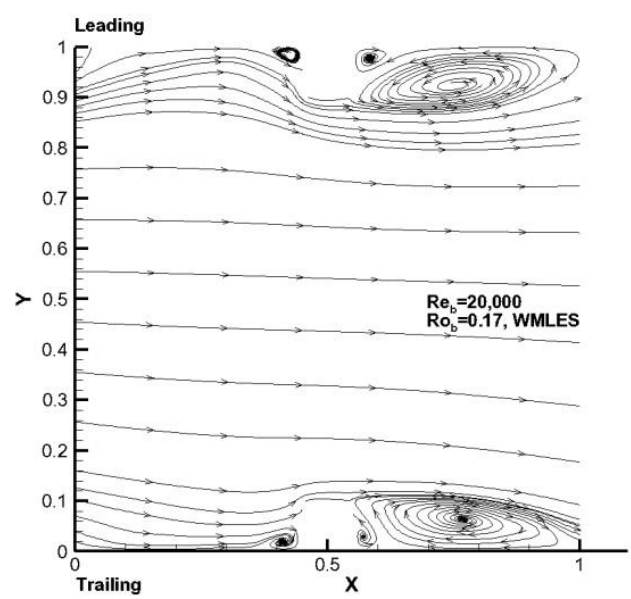

(b)

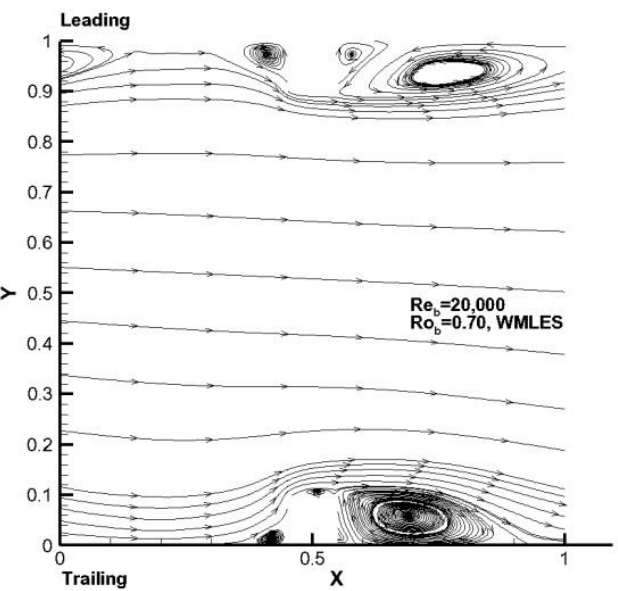

(d)

Figure 4.8 Mean flow streamlines of different rotation numbers at a center plane $(z=0.5):(a) \operatorname{Ro}=0.09$ (b) $\operatorname{Ro}=0.17$ (c) $\operatorname{Ro}=0.36$ (d) $\operatorname{Ro}=0.70$. 


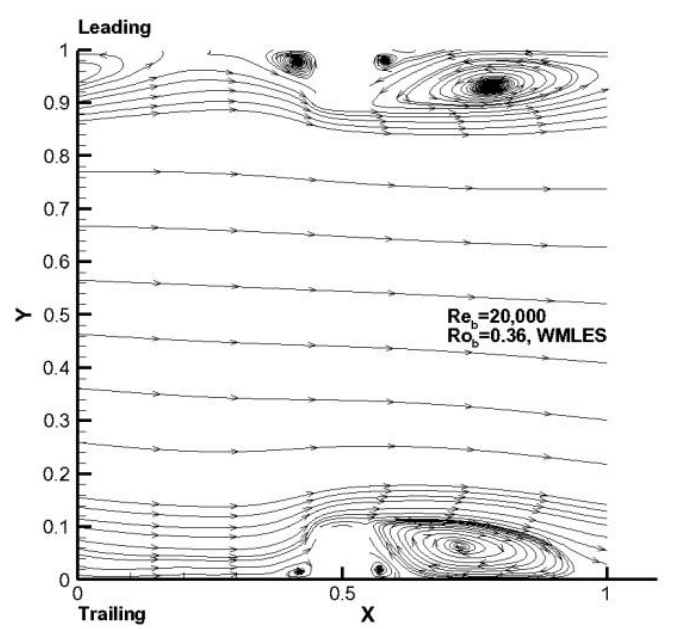

(a)

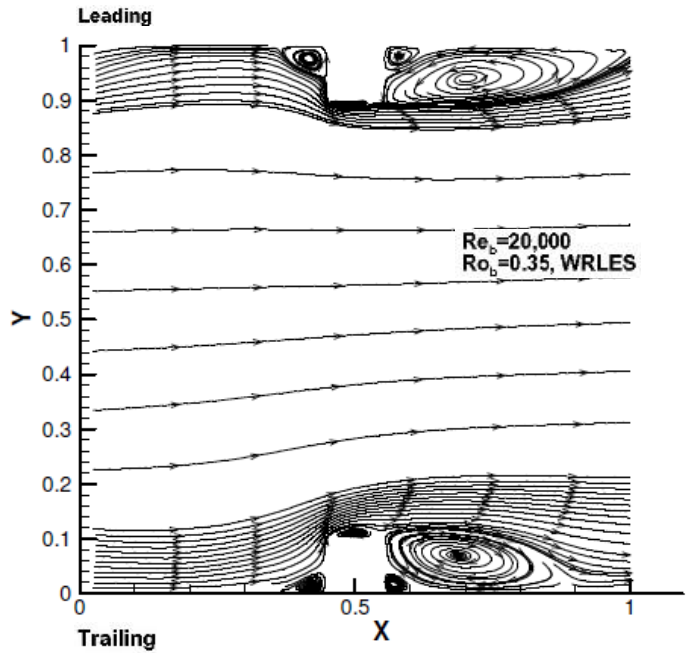

(b)

Figure 4.9 Mean flow streamlines of WMLES and LES at a center plane $(z=0.5)$ :

(a) Ro = 0.36 WMLES (b) Ro = 0.35 LES by Abdel-Wahab [25].

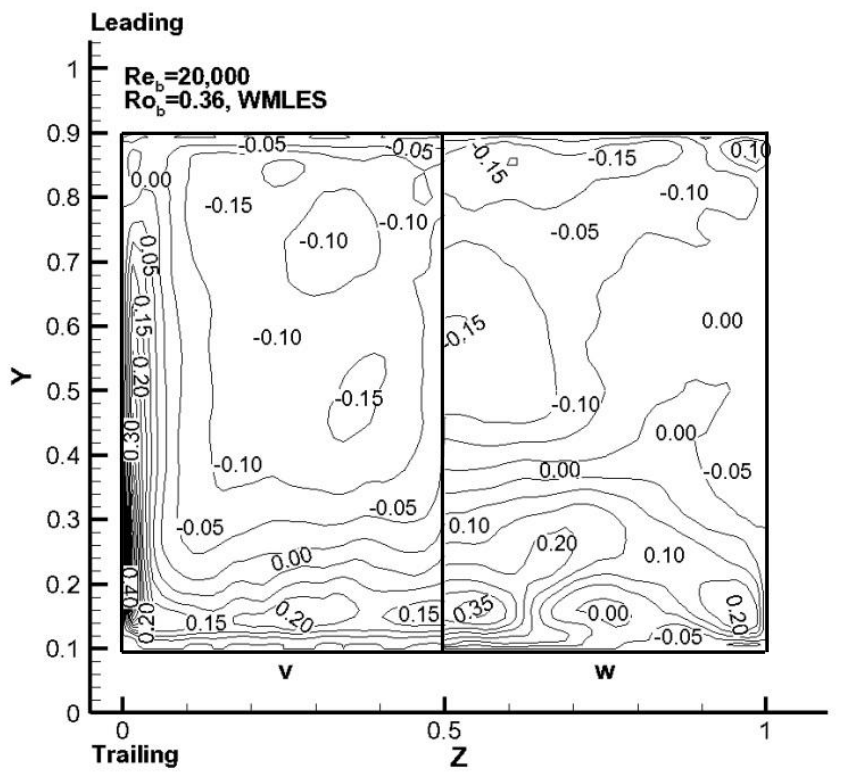

Figure 4.10 Mean wall-normal and spanwise velocities in two symmetric halves of the ribbed duct cross-section for $R=0.36$ at a rib center $(x=0.5)$. 


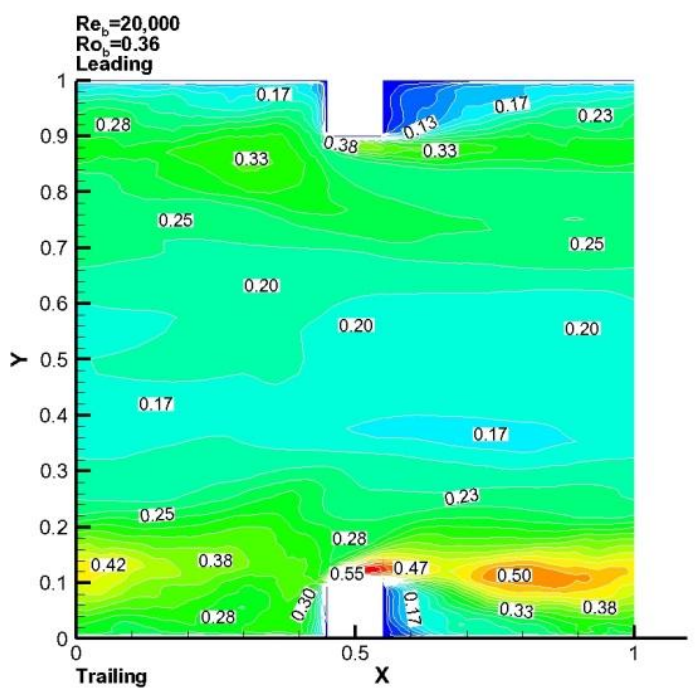

(a)

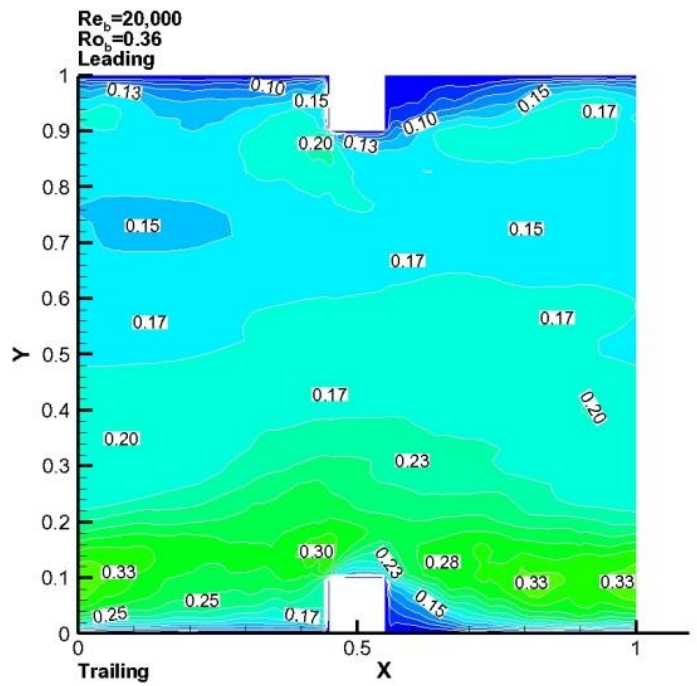

(c)

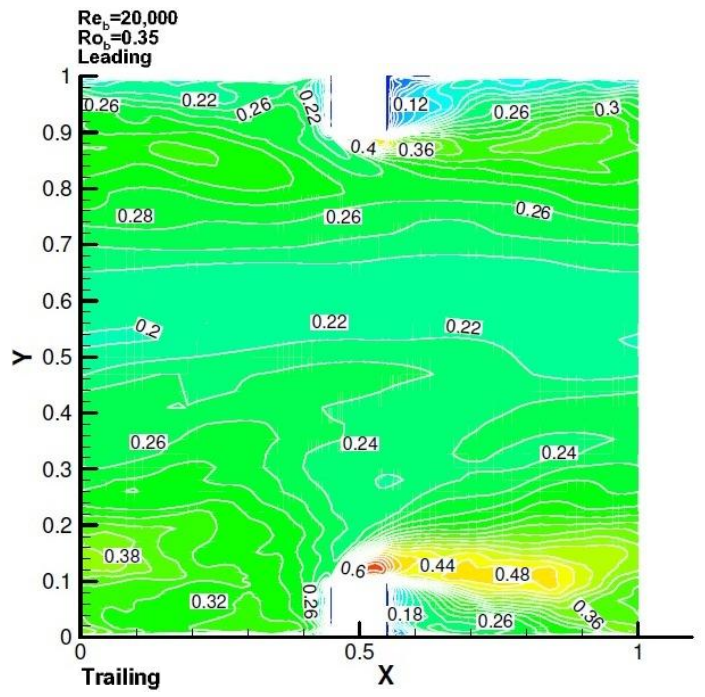

(b)

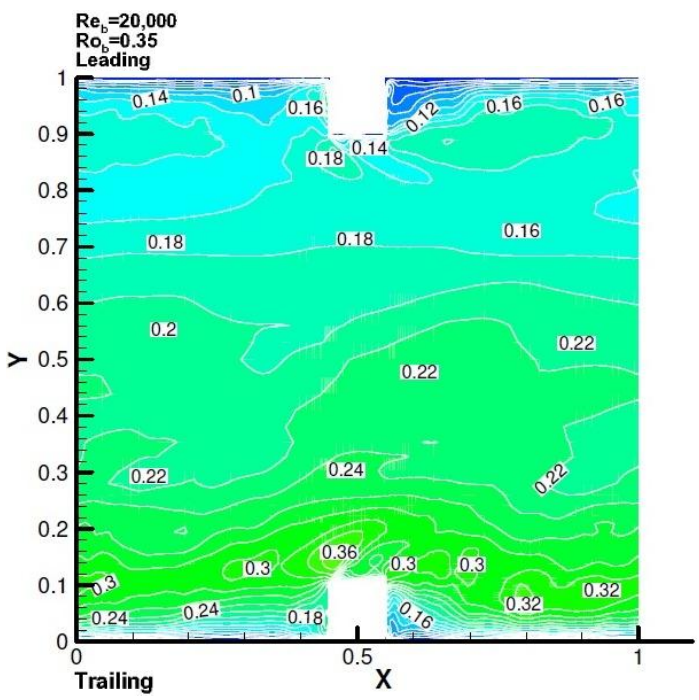

(d)

Figure 4.11 Turbulent Reynolds stresses contour plots at a center plane of the

ribbed $\operatorname{duct}(z=0.5)$ for $\operatorname{Ro}=0.36(\operatorname{Ro}=0.35$ for $\operatorname{LES}):(a) u_{r m s}$ of WMLES (b)

$u_{r m s}$ of LES (c) $v_{r m s}$ of WMLES (d) $v_{r m s}$ of LES. 


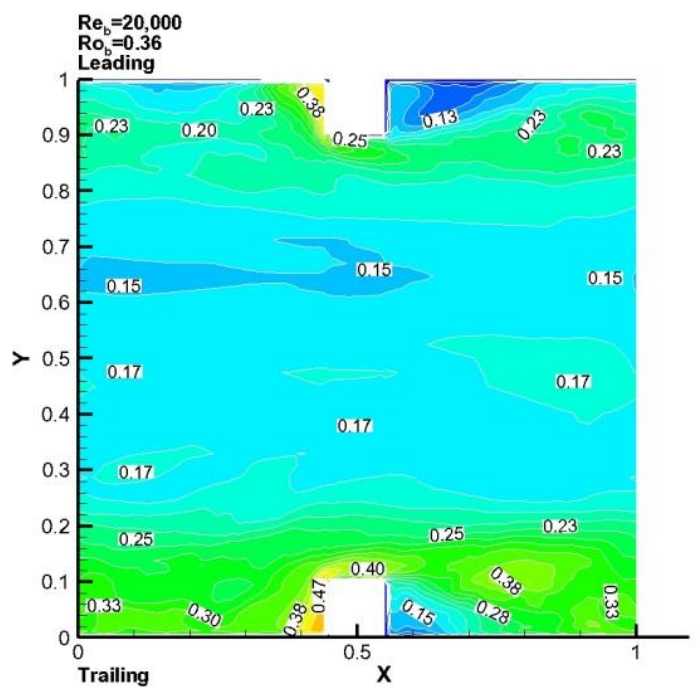

(a)

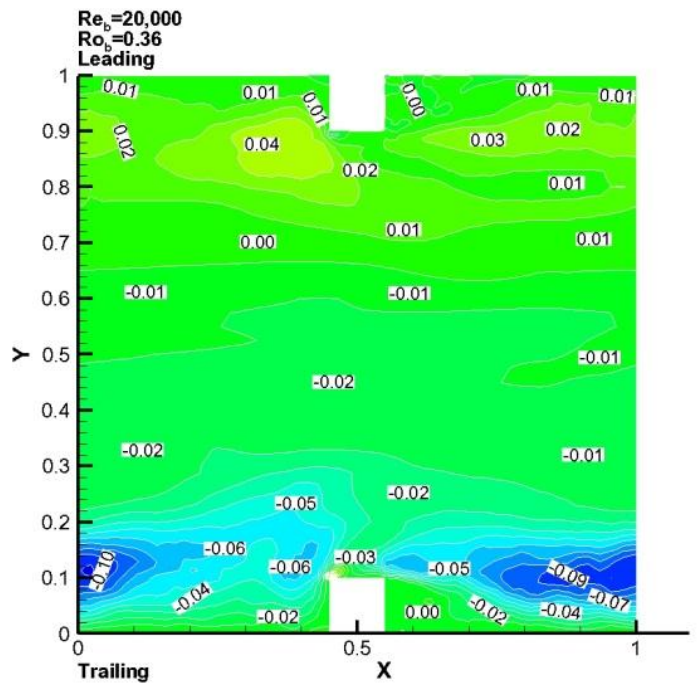

(c)

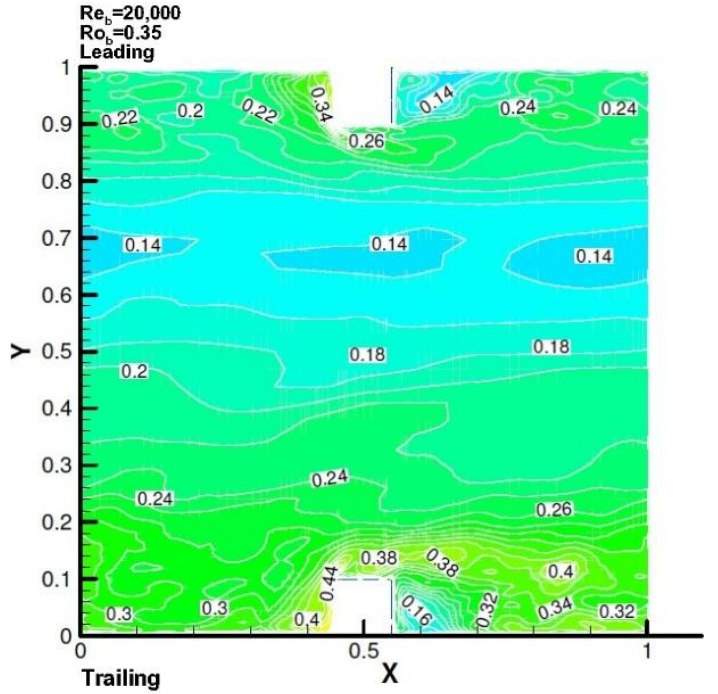

(b)

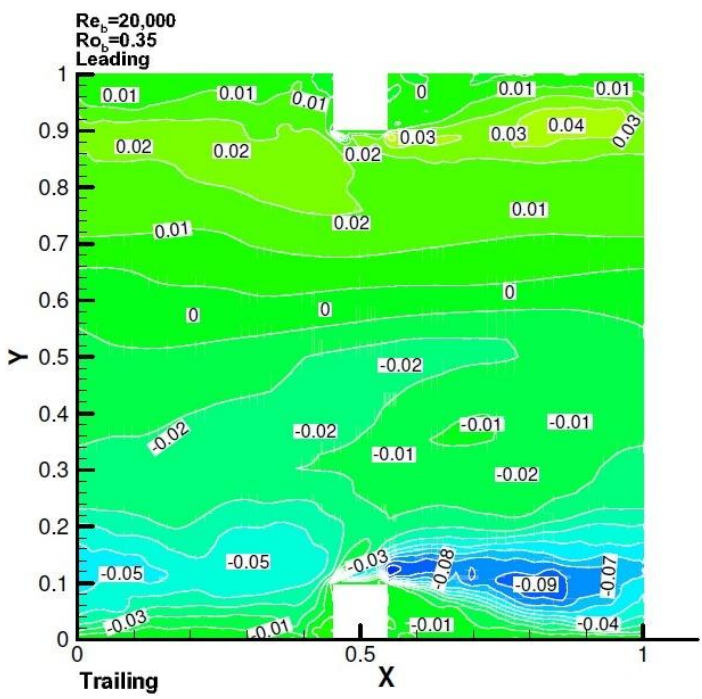

(d)

Figure 4.12 Turbulent Reynolds stresses contour plots at a center plane of the ribbed duct $(z=0.5)$ for $\operatorname{Ro}=0.36\left(\operatorname{Ro}=0.35\right.$ for LES): (a) $w_{r m s}$ of WMLES (b) $w_{r m s}$ of LES (c) $\overline{u^{\prime} v^{\prime}}$ of WMLES (d) $\overline{u^{\prime} v^{\prime}}$ of LES. 


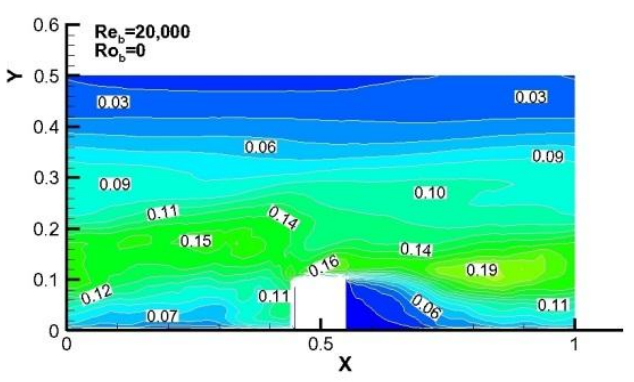

(a)

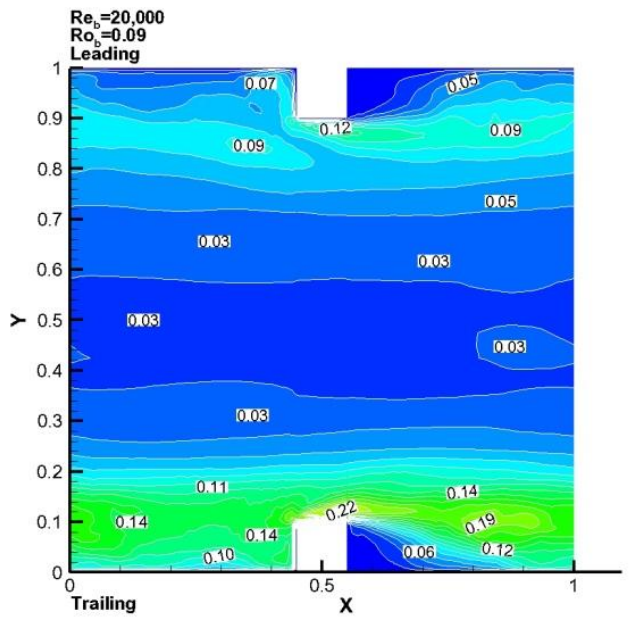

(b)

Figure 4.13 Turbulent kinetic energy (TKE) contours of different rotation numbers at a center plane $(z=0.5)$ : (a) $R o=0,(b)$ Ro $=0.09$. 


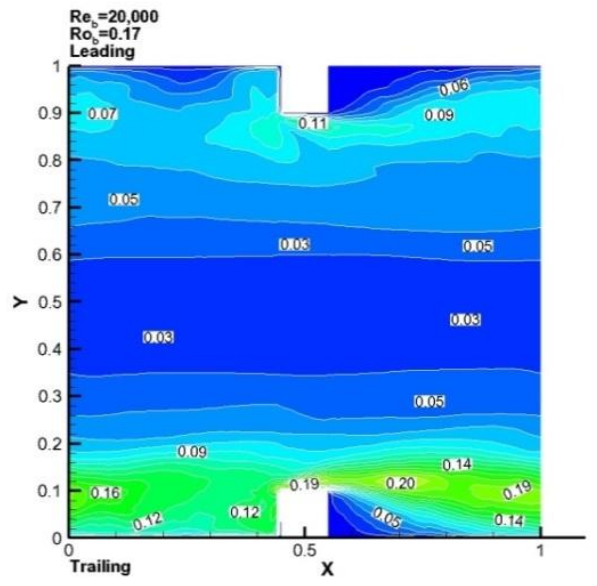

(a)

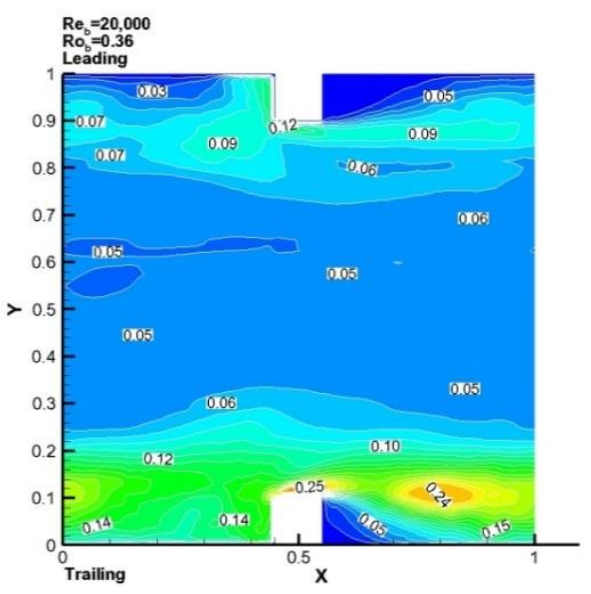

(c)

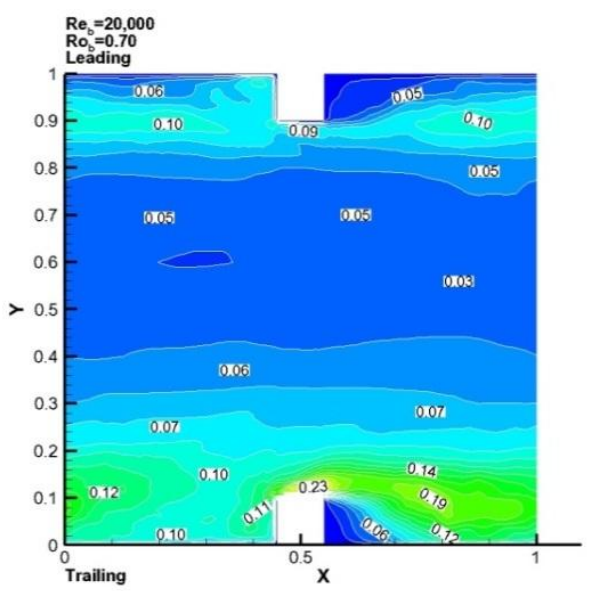

(e)

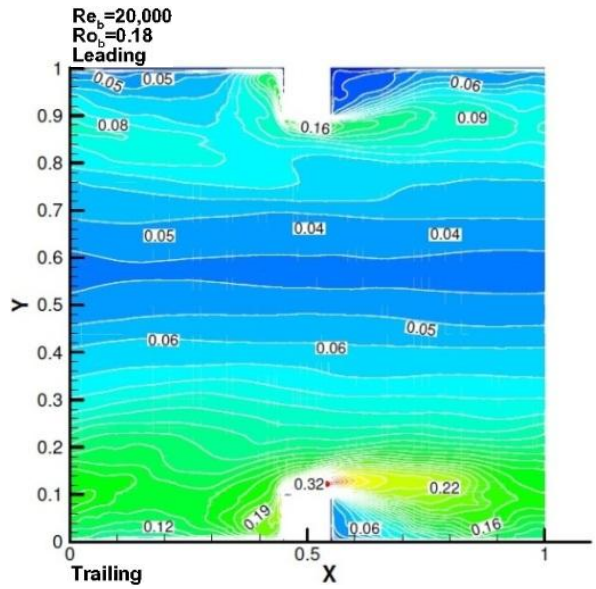

(b)

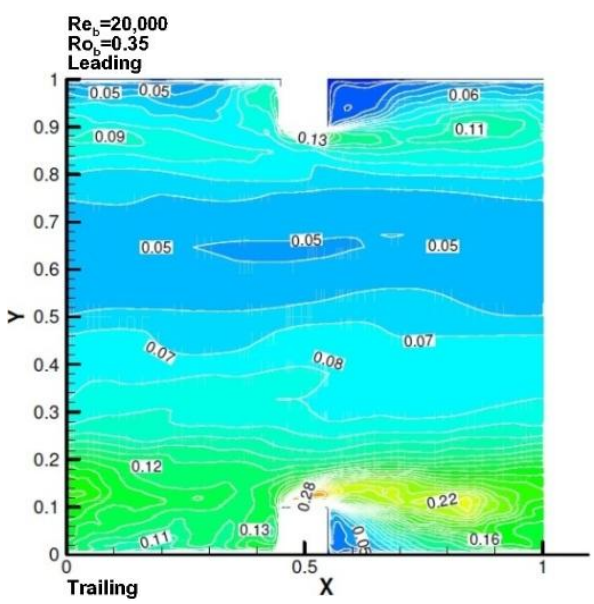

(d)

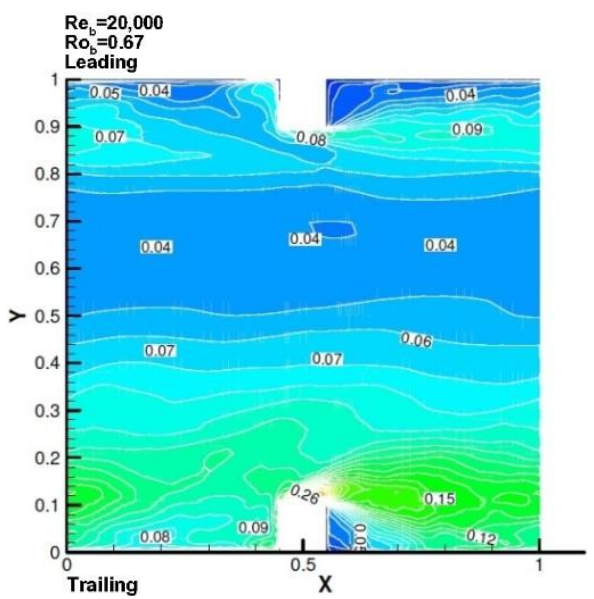

(f)

Figure 4.14 Turbulent kinetic energy (TKE) contours of different rotation numbers at a center plane $(z=0.5)$. Left column of contours (a, $c$, and e) are WMLES results whereas right column of contours $(b, d$, and $f)$ are LES results. 


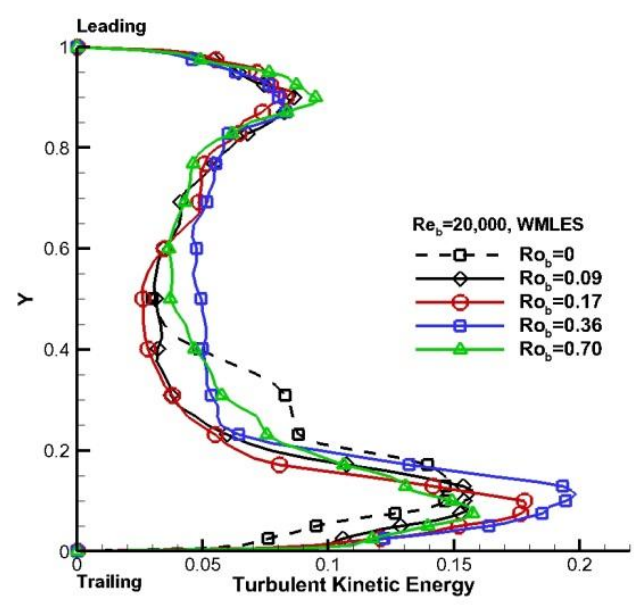

(a)

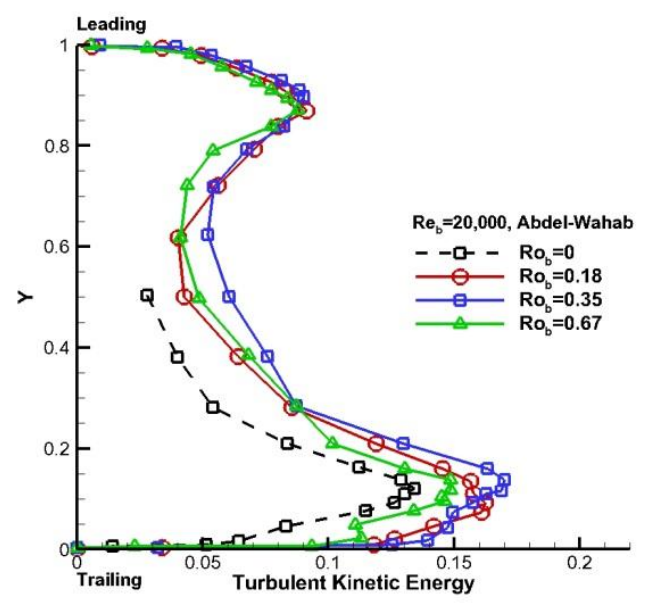

(b)

Figure 4.15 Turbulent kinetic energy (TKE) comparison plots of different rotation numbers at $x=1.0$ and $z=0.5$ : (a) WMLES results (b) LES results by Abdel-Wahab [25]. 


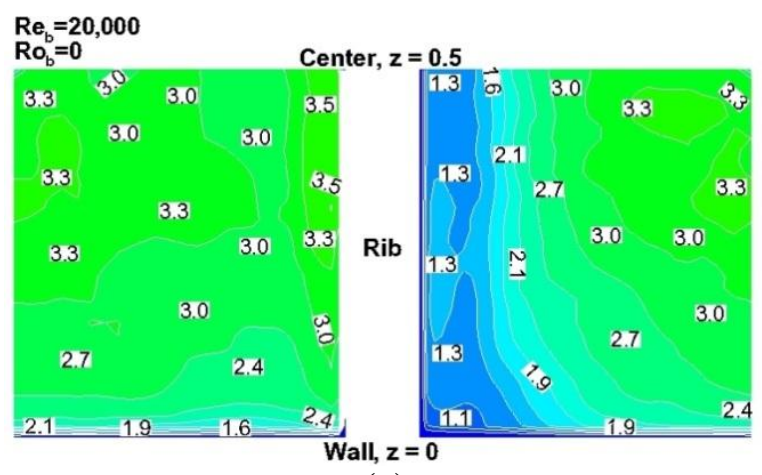

(a)

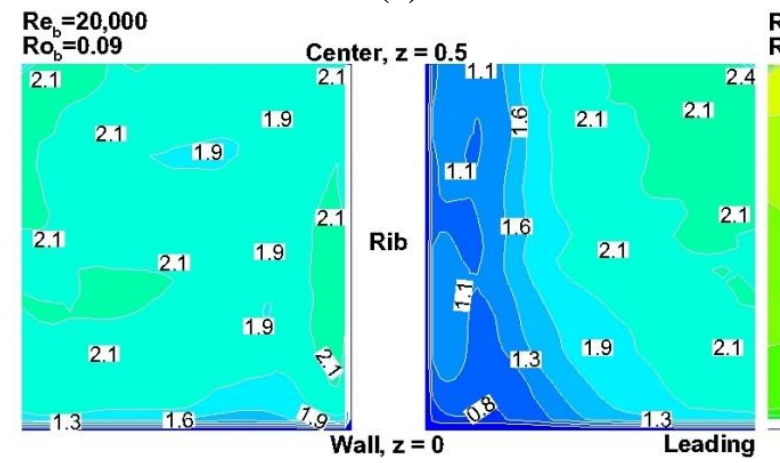

(c)

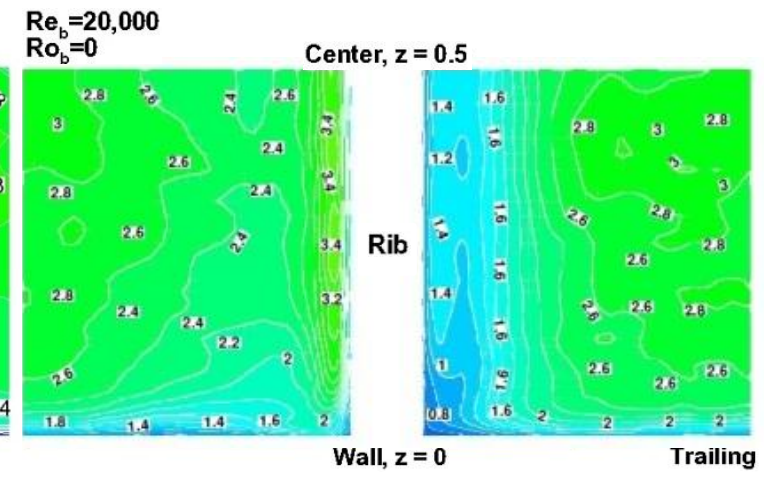

(b) $\operatorname{Re}_{\mathrm{b}}=20,000$
$\mathrm{Ro}_{\mathrm{b}}=\mathbf{0 . 0 9}$

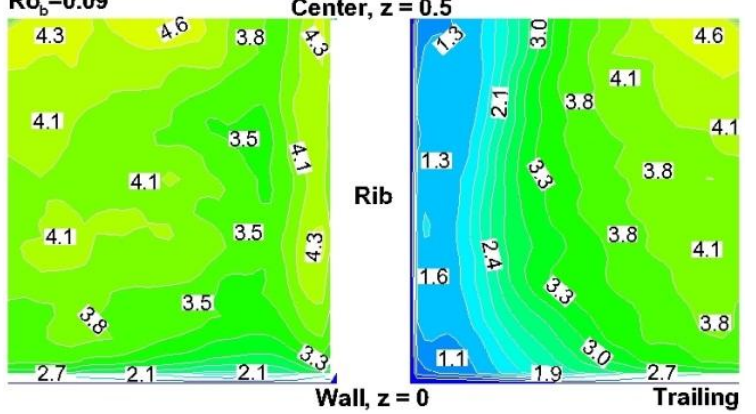

(d)

Figure 4.16 Mean Nusselt contour of half domain for the stationary case (leading and trailing sides averaged). The flow moves from left to right. (a) WMLES Ro $=0$ (b) LES Ro $=0$ (c) WMLES Ro $=0.09$ Leading (d) Ro $=0.09$ Trailing. 


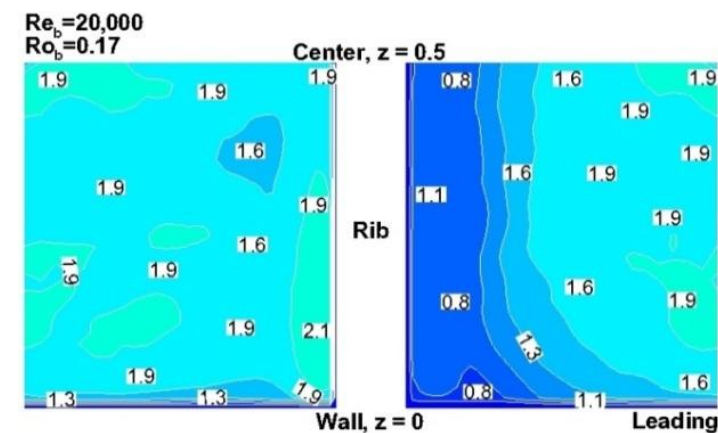

(a)

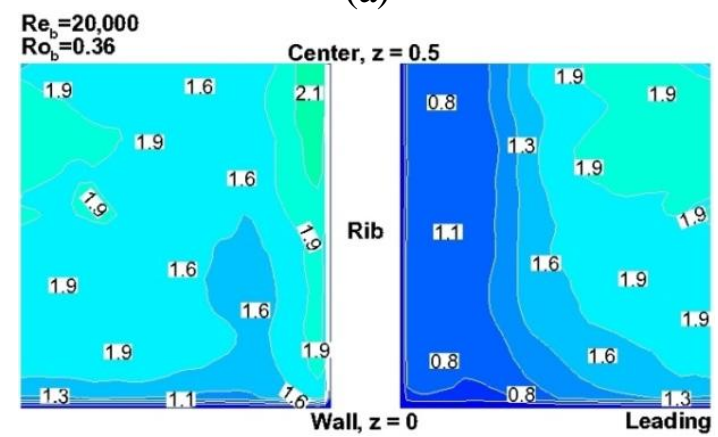

(c)

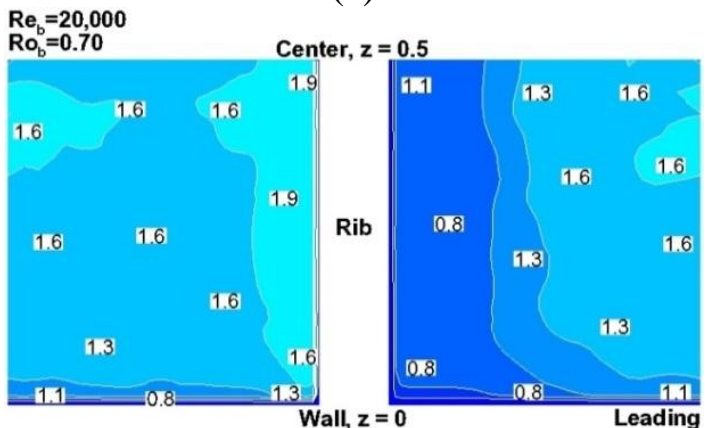

(e)

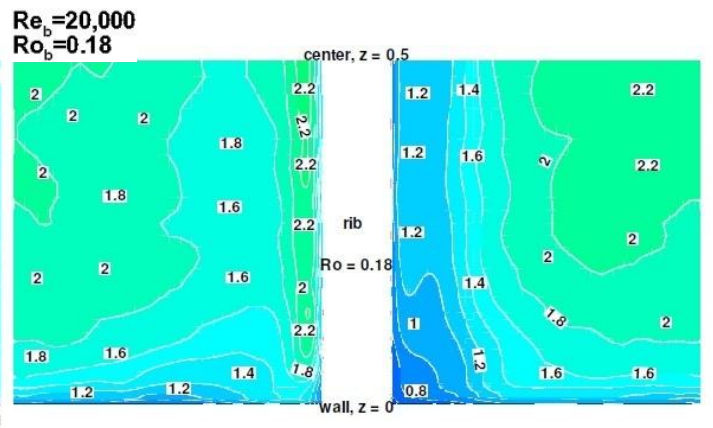

(b)

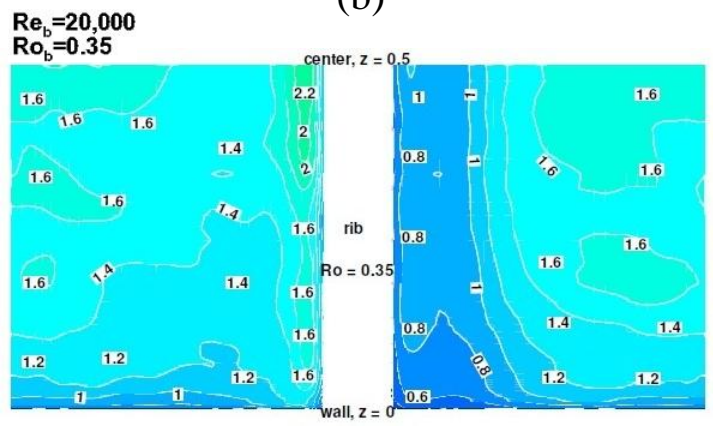

(d)

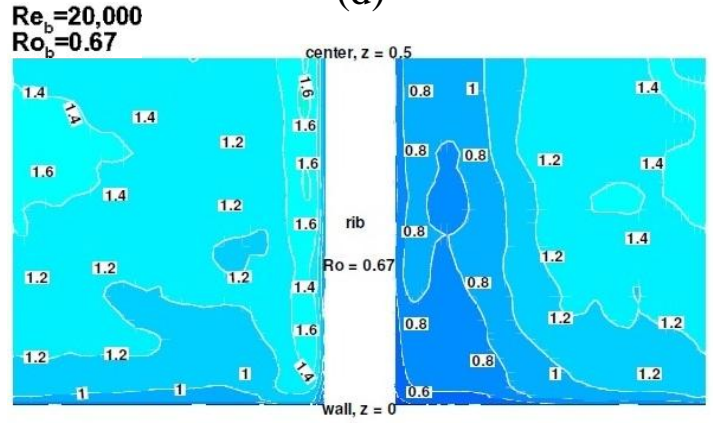

(f)

Figure 4.17 Mean Nusselt contour of half domain for different rotation numbers on leading sides. The flow moves from left to right. Left column of contours (a, $c$, and e) are WMLES results whereas right column of contours (b, $d$, and f) are LES results. 


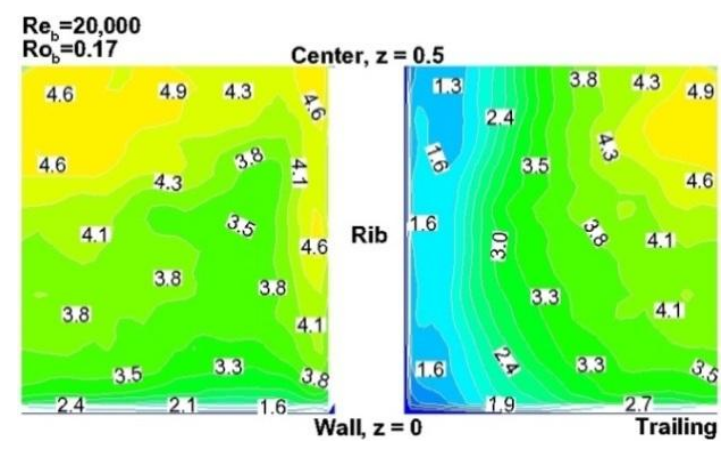

(a)

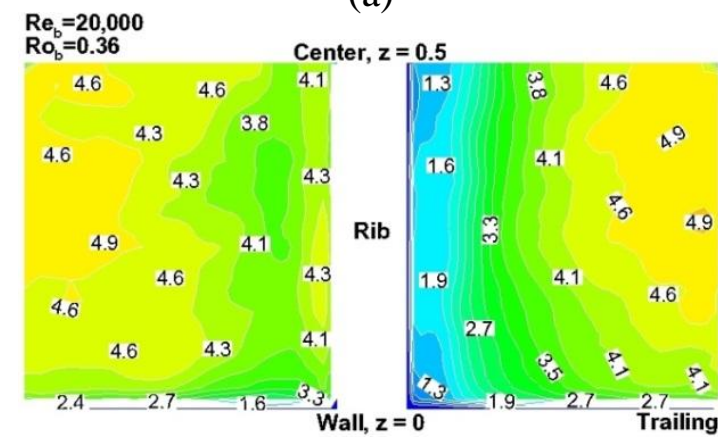

(c)

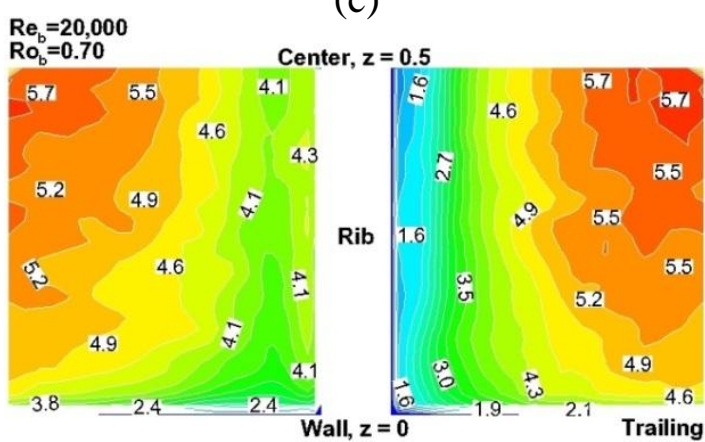

(e)

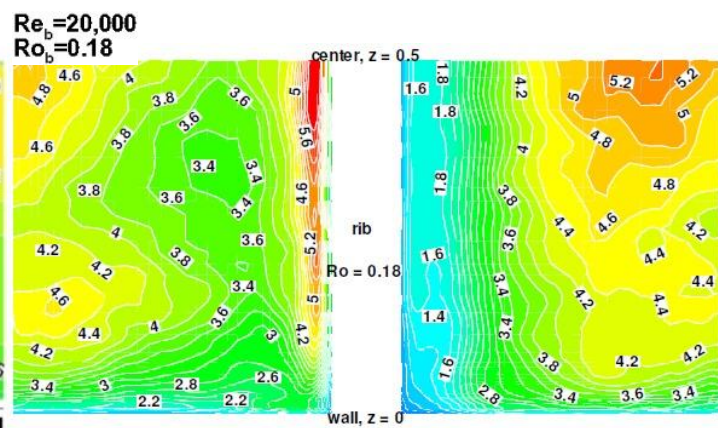

(b)

$\operatorname{Re}_{\mathrm{b}}=\mathbf{2 0 , 0 0 0}$ Ro $=0.35$

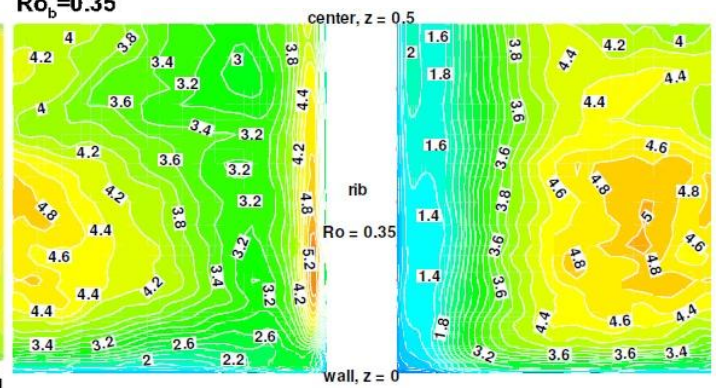

(d) $\mathrm{Ro}_{\mathrm{b}}=0.67$
$\mathrm{e}_{\mathrm{b}}=20,000$

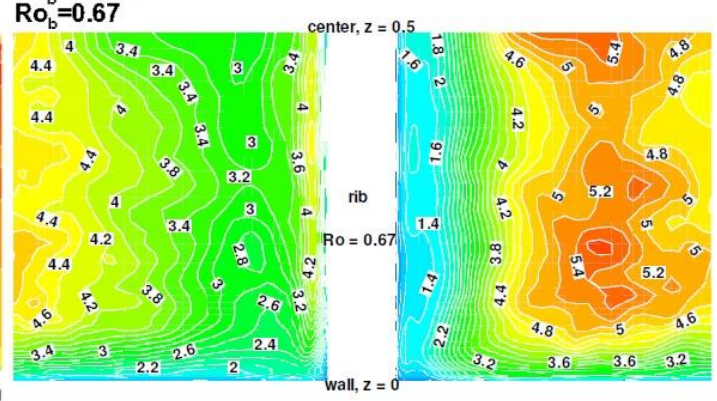

(f)

Figure 4.18 Mean Nusselt contour of half domain for different rotation numbers on trailing sides. The flow moves from left to right. Left column of contours (a, c, and e) are WMLES results whereas right column of contours (b, $d$, and f) are LES results. 


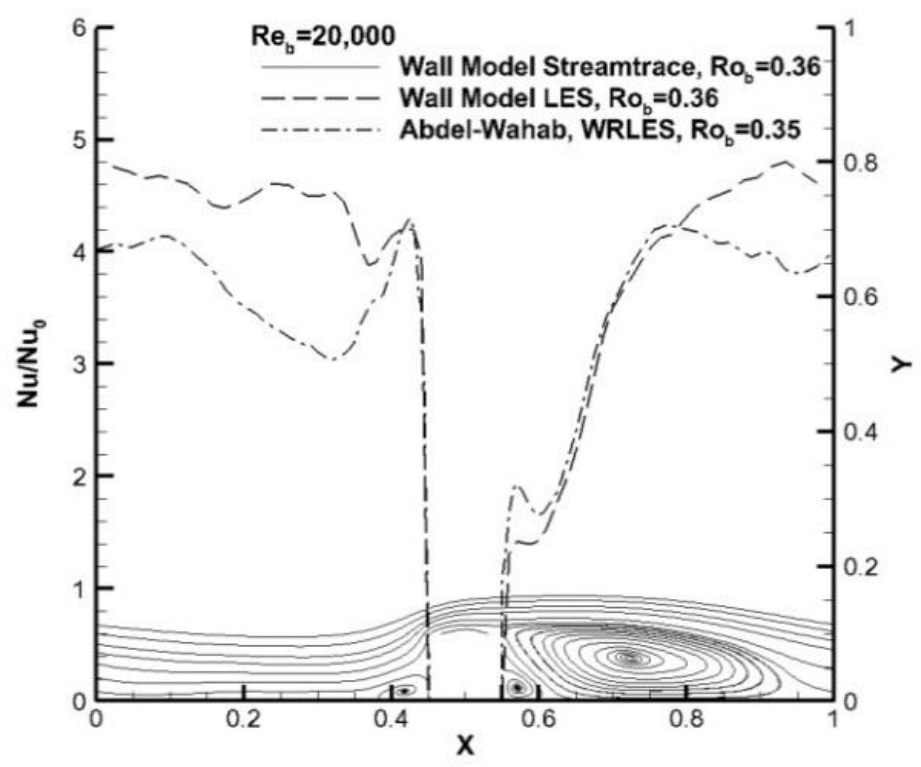

Figure 4.19 Mean Nusselt variation on the trailing side superimposed with mean flow streamlines against LES mean Nusselt variation on the trailing side by AbdelWahab $[25](z=0.5$ for mean flow streamlines). 


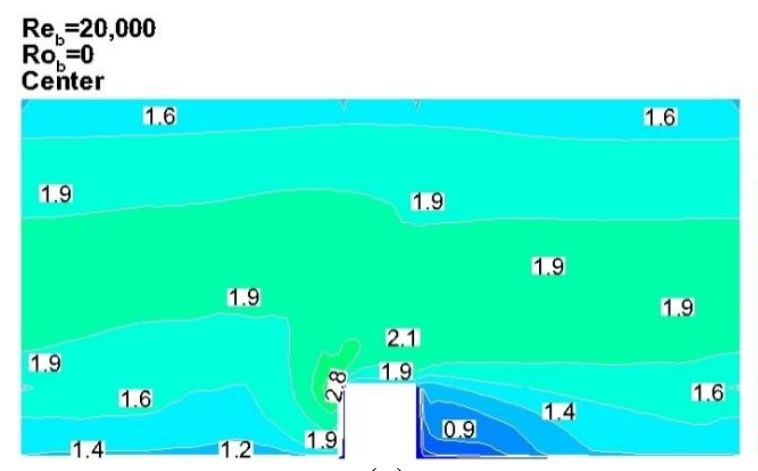

(a)

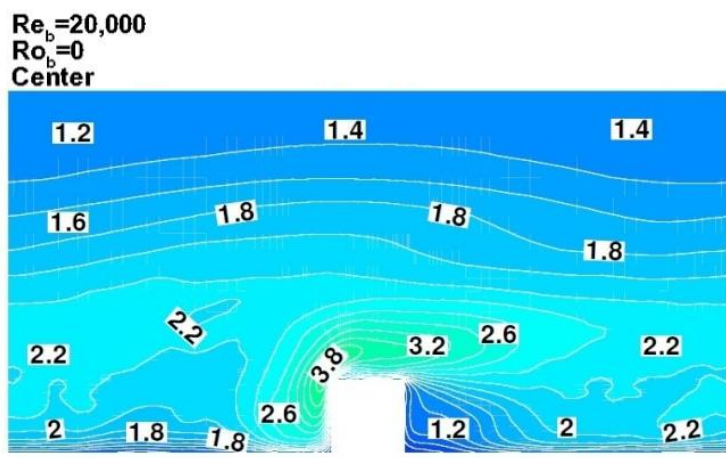

(b)

$\begin{aligned} \operatorname{Re}_{\mathrm{b}} & =20,000 \\ \mathrm{Ro} & =0.09\end{aligned}$

Reading

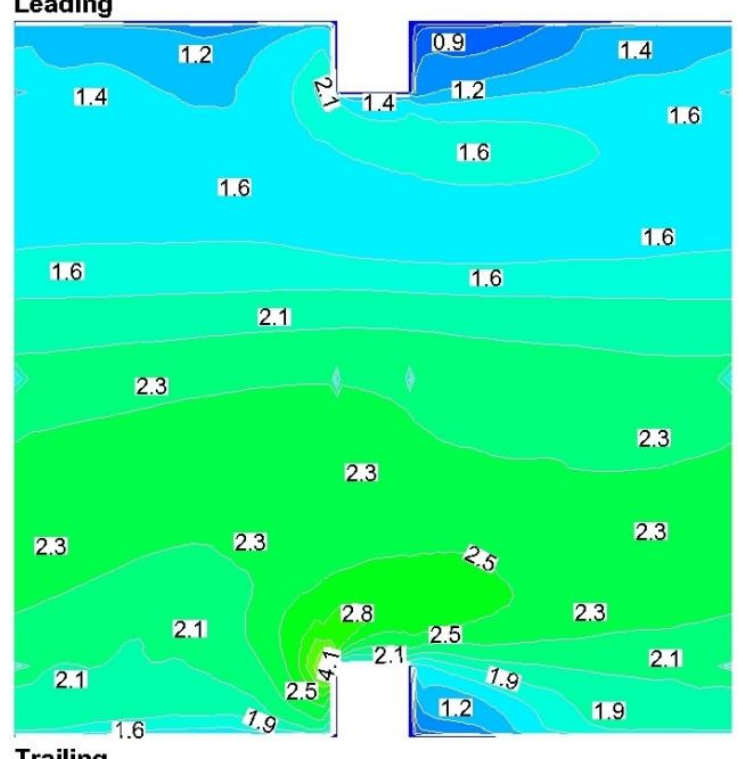

Trailing

(c)

Figure 4.20 Mean Nusselt contour of a half domain on smooth wall: (a) WMLES Ro $=0$ (b) LES Ro = 0. (c) WMLES Ro =0.09. 

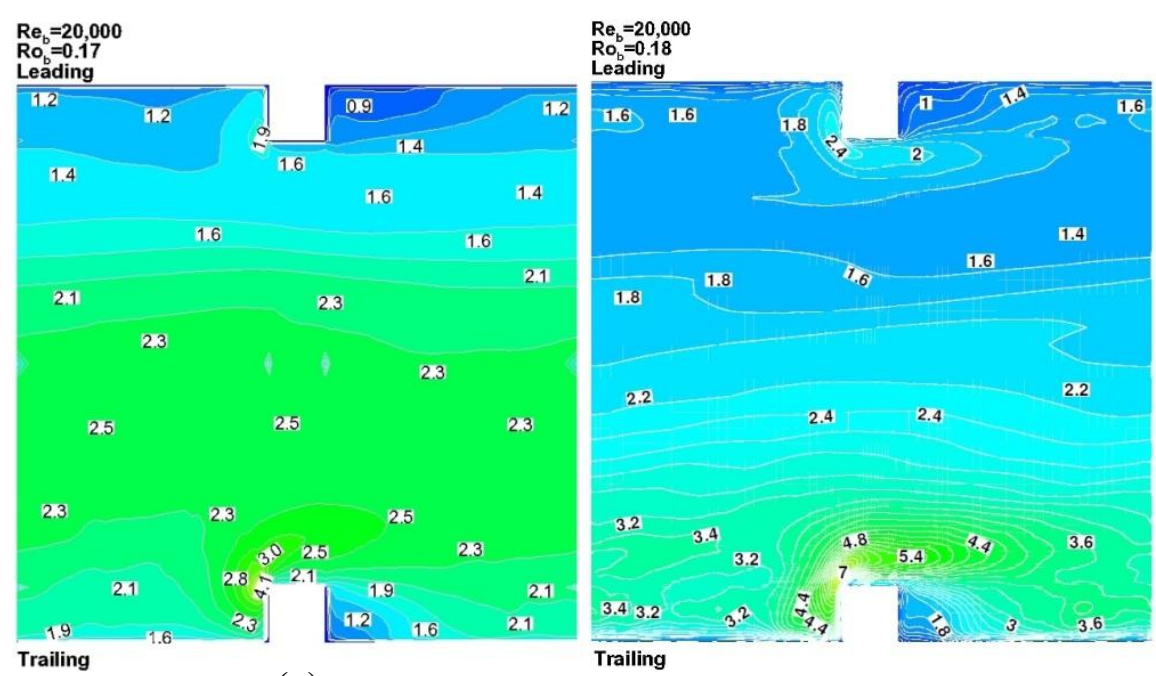

(a)

(b)
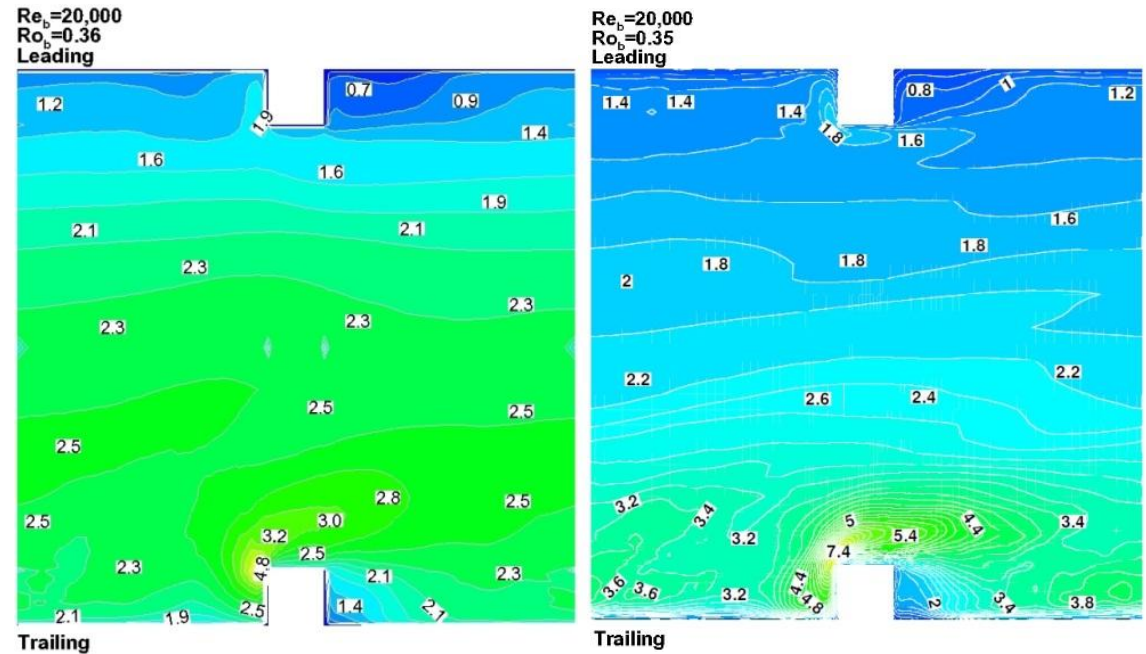

(c)

(d)

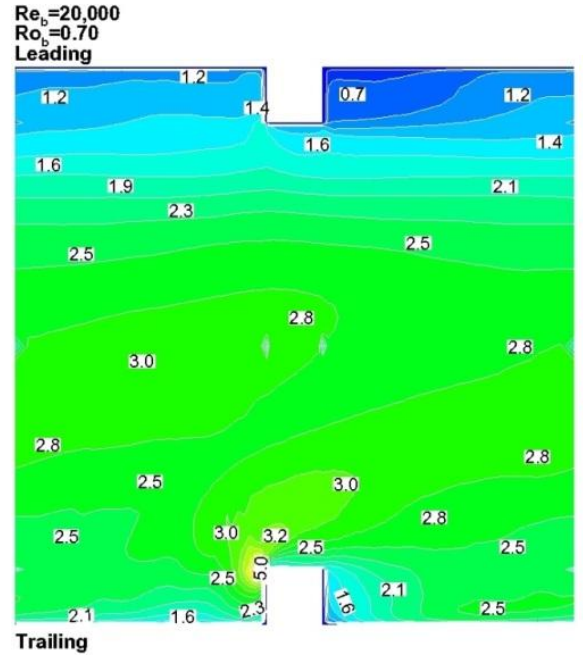

(e)

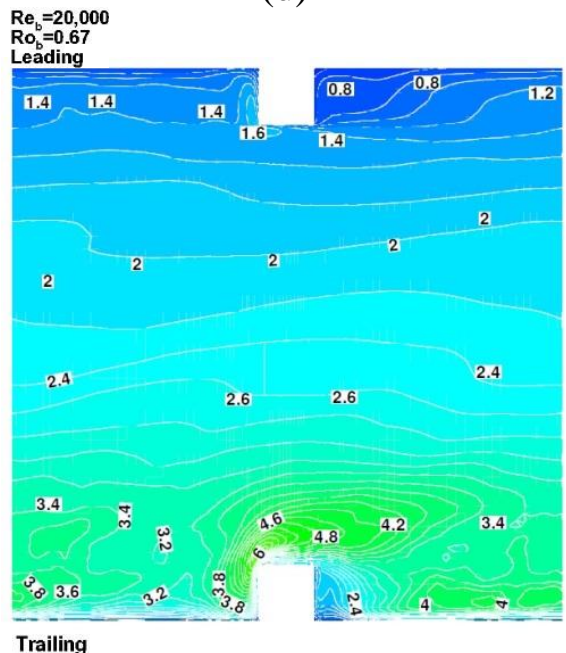

(f)

Figure 4.21 Mean augmentation contours for all rotation numbers on smooth walls:

Left column (a), (c), and (e) are WMLES, right column (b), (d), and (f) are LES. 

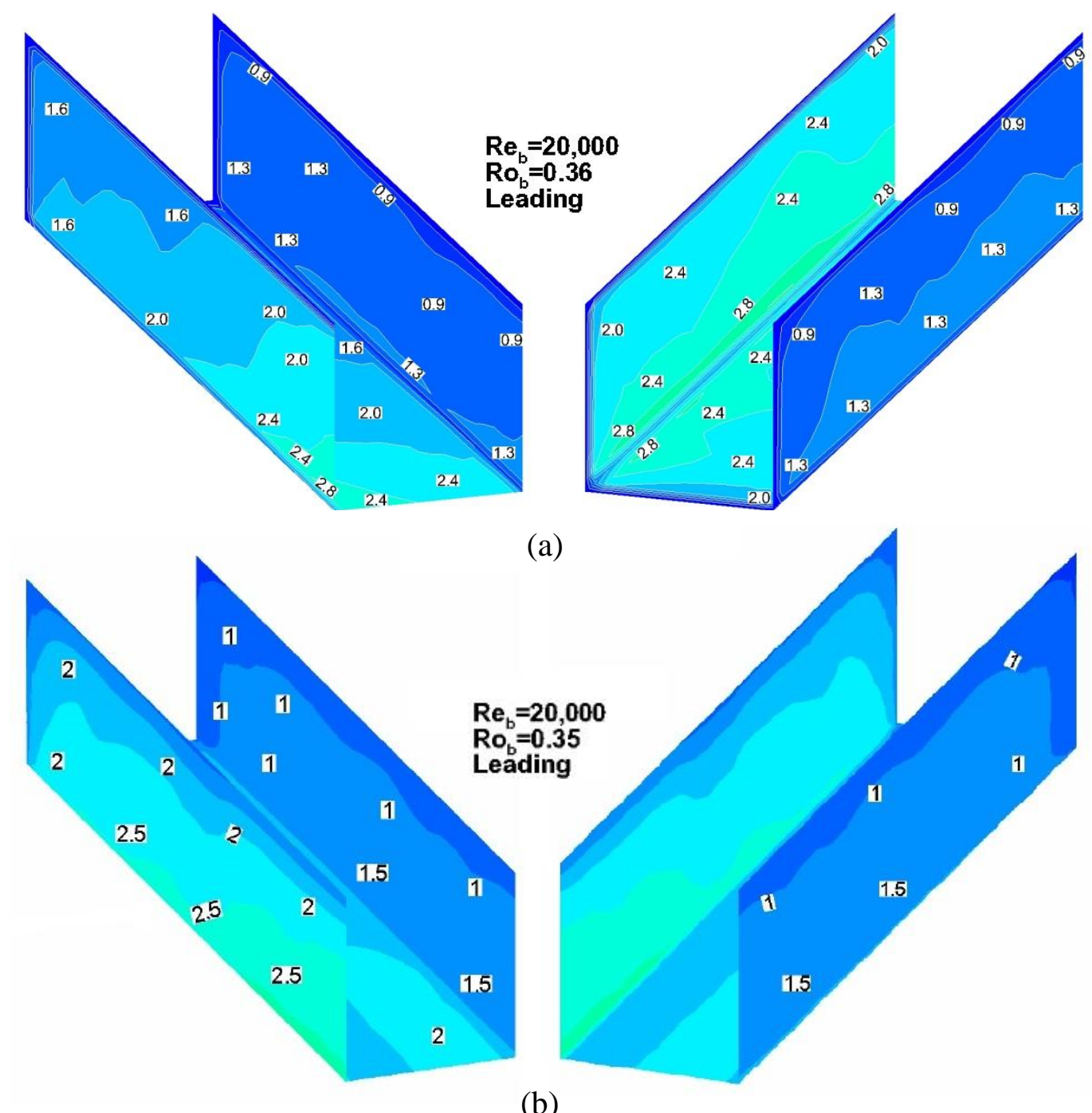

(a)

(b)

Figure 4.22 Mean Nusselt number contours split into two halves for $\mathbf{R o}=\mathbf{0 . 3 6}$ on the leading side: (a) WMLES (b) LES. 

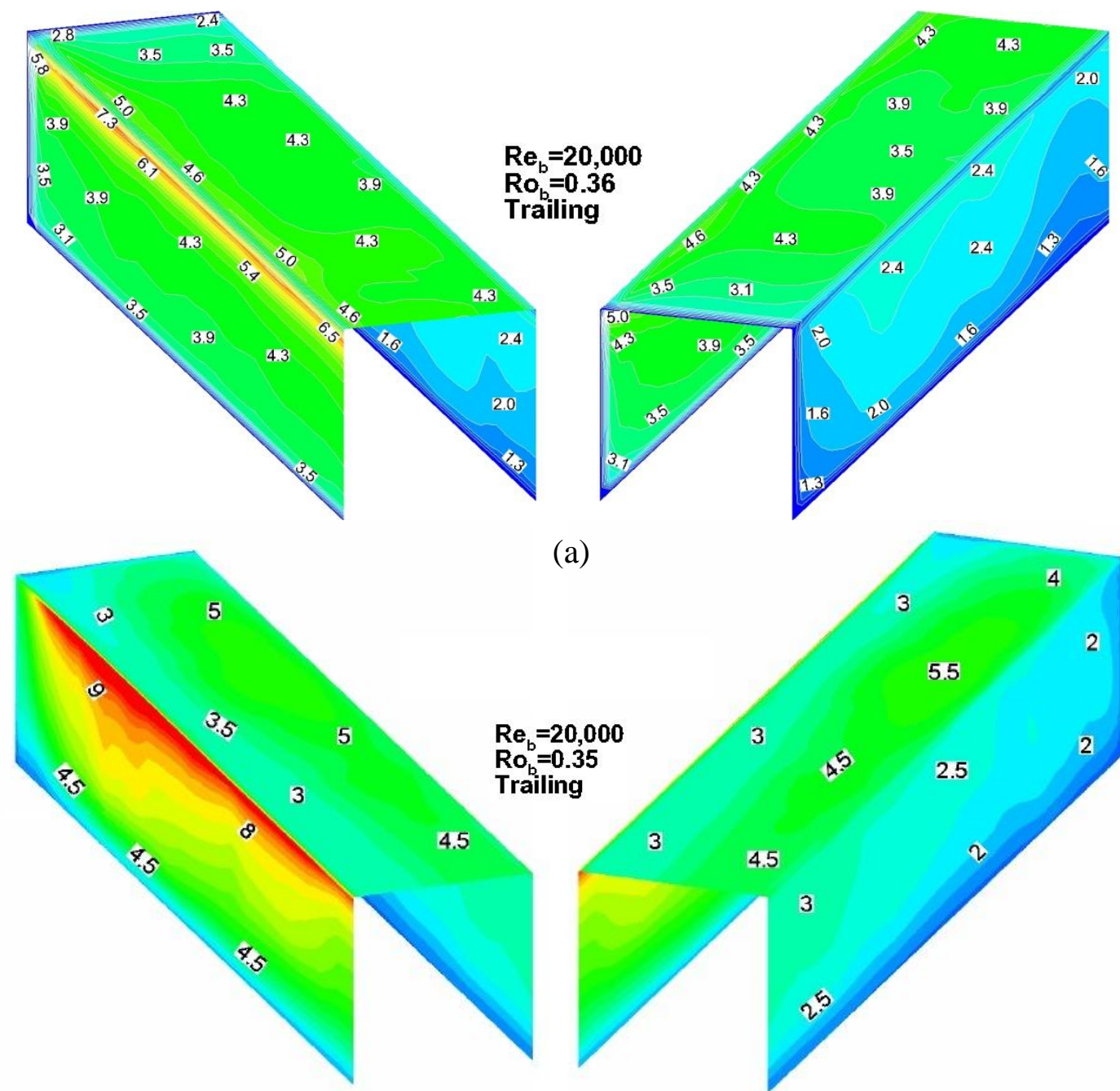

(a)

(b)

Figure 4.23 Mean Nusselt number contours split into two halves for $R=0.36$ on the leading side: (a) WMLES (b) LES. 


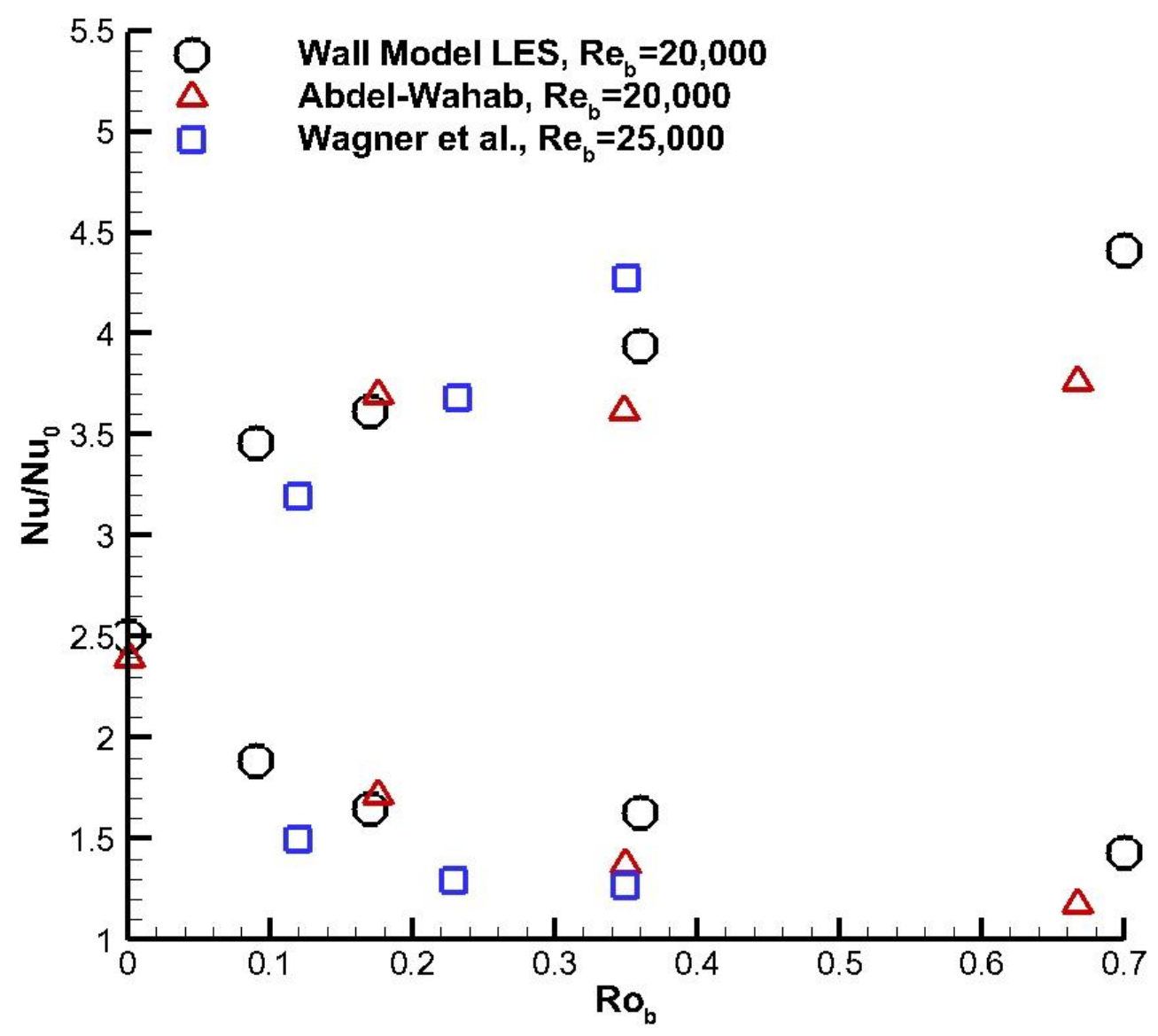

Figure 4.24 A comparison plot of WMLES, LES by Abdel-Wahab [25], and experimental data by Wagner et al. [30] for heat transfer augmentation ratios for different rotation numbers. 


\section{Chapter 5 Wall Modeled Large-Eddy Simulations of $45^{\circ}$ Staggered}

\section{Ribbed Duct with Heat Transfer}

\subsection{Introduction}

In this chapter, a stationary fully-developed flow in an angled rib duct is investigated with the wall model. The $45^{\circ}$ angled rib is characterized by strong secondary flows, which would test the robustness and prediction accuracy of the wall model at a high bulk Reynolds number of 49,000. Both velocity and heat transfer results are calculated and compared to the experimental data of Chanteloup et al. [33] and LES results by Abdel-Wahab [25] with grid resolution $\Delta \mathrm{y}_{1}{ }^{+}<1$. The simulations are performed at a bulk Reynolds number of 49,000 with (rib height to hydraulic diameter ratio) $e / D_{h}$ of 0.1 and (rib pitch to height ratio) $p / e$ of 10 rib parameters.

The $45^{\circ}$ angled rib orientation causes the formation of helical vortices behind the ribs, and strong secondary flow motion in the cross-section of the duct. The effect of these motions on turbulence and heat transfer augmentation is investigated. High heat transfer is found at the origin of the helical vortex when it is close proximity to the surface and in the region where the secondary flow impinges on the side walls of the duct. The results compare reasonably well with previous LES and experiments, except the secondary flow structure, which is found to be biased toward one side of the duct. 


\subsection{Literature Review}

Liou et al. [34] investigated a $45^{\circ}$ rotating rib-roughened rectangular duct with an aspect ratio of 0.5 at 5,000 to 15,000 bulk Reynolds numbers, 0 to 2 bulk rotation numbers, and 0.07 to 0.28 density ratios. The staggered rib setup included $e / D_{h}$ of 0.1 and p/e of 10 rib parameters where a constant heat flux boundary condition was used in the experiment. Compared to the Dittus-Boelter relation, the heat transfer augmentation was enhanced by 1.6 to 4.3 times relative to a stationary case. As the bulk Reynolds number increases, secondary flow motions, or flow impingements, enhance fluid mixing resulting in higher heat transfer values. However, a decrease in heat transfer values was evident with increase in bulk Reynolds numbers.

Han et al. [35] performed experiments on $90,75,60,45,30$, and $15^{\circ}$ angled ribs over bulk Reynolds numbers of 7,000 to 90,000 . The $e / D_{h}$ of 0.063 was maintained where p/e of 10 to 20 was varied based on the rib angles. The test section included 36 thermocouples where 4 heated parallel aluminum plates were used as heat sources. Based on a series of data points, correlations of friction factors and Nusselt numbers were found, which accounted for rib height, rib spacing, rib angle, and bulk Reynolds numbers. The study indicated that for $p / e$ of 10 , a $90^{\circ}$ angled rib enhanced the average Nusselt number by approximately 2 times and average friction factor by 4 to 6 times compared to a smooth duct geometry. In particular, a $45^{\circ}$ angled rib showed 25\% higher Nusselt number compared to a $90^{\circ}$ angled rib where $p / e$ of 10 indicated better performance than $p / e$ of 20 . Furthermore, a $45^{\circ}$ angled rib with $p / e$ of 10 resulted in higher efficiency compared to other rib parameter combinations where the efficiency was calculated based on the pumping power requirement for different rib parameters. 
In addition, Han and Park [36] studied 90, 60, 45, and $30^{\circ}$ angled ribs for bulk Reynolds numbers from 10,000 to 60,000 with aspect ratios of 1,2 , and 4 . The $e / D_{h}$ of 0.047 was used for aspect ratios of 1 and 2 whereas the $e / D_{h}$ of 0.078 was used for an aspect ratio of 4 with p/e of 10 to 20 variations for different rib angles and bulk Reynolds numbers. Compared to the previous study by Han et al. [35], which used an aspect ratio of 1 , the study showed as much as $30 \%$ increase in heat transfer values for 30 to $45^{\circ}$ angled ribs with aspect ratios of 2 and 4 relative to the transverse ribs $\left(90^{\circ}\right)$ while maintaining the pumping power for all cases. In addition, the secondary flow motion near top and bottom walls reflected the direction of the impingements due to vortices generated by angled ribs where heat transfer values were significantly affected by secondary flow motion in downstream $\left(x / D_{h}>3\right)$.

Bonhoff et al. [37] combined efforts to validate experimental data with 3 different turbulence models using FLUENT. A bulk Reynolds number of 50,000,e/D $h_{h}$ of 0.1 , and p/e of 10 were used in a staggered rib setup. Particle image velocimetry (PIV) was used to measure 3 mean velocity components and 6 Reynolds stresses. A structured mesh was used in these simulations where the Reynolds-averaged Navier-Stokes (RANS) methodology was used with 3 different turbulence models: the standard $k-\varepsilon$ model with wall functions, the differential Reynolds stress model (RSM) with wall functions, and two-layer $k-\varepsilon$ model. Compared to the experimental data in velocity profiles, streamwise velocity profiles were overpredicted whereas wall-normal velocity profiles were underpredicted in simulations. However, spanwise velocity profiles showed reasonable agreements in the outer layer. In addition, friction factor, Nusselt number, and efficiency values were compared against the correlation found in Han et al. [35]. The 
results indicated that RSM with wall functions showed relatively favorable results compared to other CFD simulations. Details of the experimental and computational setup are further discussed in Bonhoff et al. [37].

Similarly, Chanteloup et al. [33] did a study on $45^{\circ}$ angled ribs at a bulk Reynolds number of 50,000 with $e / D_{h}$ of 0.1 , and $p / e$ of 10 . Investigations were done based on the previous study by Han and Park [36]. Also, the $16^{\text {th }}$ rib module was shown to be fullydeveloped where all measurements were taken downstream of this region. For heat transfer measurements, a transient liquid crystal technique was used to monitor the surface temperature where thermocouples were placed along the passage to measure the gas temperature. In addition, PIV and heat transfer uncertainty analysis were done based on other literature mentioned in this paper, which indicated an uncertainty of within $10 \%$. Compared to the friction factor by Han and Park [36], the result indicated as much as $30 \%$ difference, which appeared to be due to a difference in aspect ratio. The flow characteristics showed that the secondary flow motion consisted of 2 counter-circulating vortices along the spanwise direction where the flow impingements due to the rib orientation showed as high as 0.3 of $u_{b}$ on the outer wall at approximately 1 rib height above the ribbed walls. Also, heat transfer augmentation ratios were the highest behind the rib towards the inner wall as much as 6 times the Dittus-Boelter correlation value of the smooth duct. Furthermore, high heat transfer region on the outer wall due to flow impingements induced by vortex generations by the angled ribs was also found.

Abdel-Wahab [25] simulated $45^{\circ}$ angled ribbed duct case at a bulk Reynolds number of approximately 47,000. LES was used to simulate the case with experimental data by Chanteloup et al. [33] to validate results. The mesh size was $160 \times 128 \times 128$ in 
$\mathrm{x}, \mathrm{y}$, and $\mathrm{z}$ directions where the first mesh point was placed at $\Delta^{+}<1$ with 4 to 5 mesh points placed within 10 wall units. Compared to experimental data, LES results indicated less than $15 \%$ difference in heat transfer values and a close match against velocity profiles.

\subsection{Computational Model and Details}

The fully-developed computational model in Chapter 2.3 is used to simulate one pitch of the duct with two staggered ribs. A nominal bulk Reynolds number, $R e_{b}$ of 49,000 is simulated with and without the wall model. $R e_{b}$ is defined as follows:

$R e_{b}=\frac{\bar{u}_{b} D_{h}}{v}$

where $\bar{u}_{b}$ is the time-averaged bulk velocity, $D_{h}$ is a hydraulic diameter, and $v$ is the kinematic viscosity of the medium, which is air for all cases.

The computational domain for the ribbed duct simulations is presented in Figure 5.1. The computational domain consists of approximately $6.4 \times 10^{5}$ cells, or $100 \times 80 \times$ 80 cells in $\mathrm{i}, \mathrm{j}$, and $\mathrm{k}$ directions where $\mathrm{i}$ is the streamwise direction, $\mathrm{j}$ is the wall normal direction, and $\mathrm{k}$ is the spanwise direction. A non-uniform grid distribution is used in $\mathrm{x}, \mathrm{y}$, and $\mathrm{z}$ directions with spacing values of approximately $0.00176,0.00176$, and 0.00462 , respectively, which are equivalent to $\Delta x_{1}{ }^{+}$and $\Delta y_{1}{ }^{+}$values of 16 and a $\Delta z^{+}$value of 43 . These values were based on the specified $R e_{\tau}$, which is approximately 18,610 . The $e / D_{h}$ of 0.1 and p/e of 10 are used for all simulations as previously mentioned. Wall boundary conditions are set on both xy- and xz-planes whereas periodic boundary conditions are set on yz-planes. A constant heat flux is specified at all walls and the ribs. 
The non-dimensional time step value of $1 \times 10^{-4}$ is used and time averaging is done for 5 non-dimensional time units to obtain mean quantities. The momentum and pressure equations are solved implicitly to the convergence criteria value of $1 \times 10^{-6}$ at each time step. All ribbed duct cases utilize 4 cores of an Intel Xeon Linux cluster. Each time step takes approximately $12.8 \mu$ s of wall clock time/grid node.

For the heat transfer analysis, the local Nusselt number is calculated as:

$N u=\frac{1}{\theta_{s}-\theta_{\text {ref }}}$

where $\theta_{s}$ is the surface temperature and $\theta_{\text {ref }}$ is the reference temperature. The reference temperature is calculated based on the averaged temperature field across the geometry:

$\theta_{\text {ref }}=\frac{\iint\left|u_{1}\right| \theta d A_{x}}{\iint\left|u_{1}\right| d A_{x}}$

Then, the averaged Nusselt number is calculated based on the local Nusselt number:

$\overline{N u}=\frac{1}{\iint_{\Omega} d S}\left[\iint_{\Omega} \frac{1}{\theta_{s}-\theta_{\text {ref }}} d S\right]$

where $S$ is the appropriate surface for the averaged Nusselt number. In addition, the averaged Nusselt number is normalized by the Dittus-Boelter equation:

$N u_{0}=0.023 \cdot R e_{b}^{0.8} \cdot \operatorname{Pr}^{0.4}$

The Fanning friction factor that is used for the ribbed duct analysis is defined as follows:

$f=\frac{1}{2 \cdot \bar{u}_{b}^{2}}$

All quantities are non-dimensionalized by $u_{b}$ or $u_{b}^{2}$ unless specified where $u_{b}$ is equivalent to $U_{\text {mean }}$. Also, Reynolds number $(R e)$ is equivalent to the bulk Reynolds number, $R e_{b}$. 


\subsection{Results of $45^{\circ}$ Ribbed Duct at $\operatorname{Re}=49,000$}

The results of WMLES was compared to LES results by Abdel-Wahab [25] and experimental data by Chanteloup et al. [33] as seen in Table 5.1. While form drag and frictional losses do not indicate significant differences, heat transfer augmentation ratios indicate mixed results compared to LES and experimental results. While heat transfer augmentation ratio of ribbed walls lie between LES and experimental results, heat transfer augmentation ratios on the ribs are underpredicted. On the other hand, the augmentation is overpredicted at the inner and outer walls. These prediction trends are similar to those observed in orthogonal ribbed ducts. Overall heat transfer with wall treatment shows results within $6 \%$ of the experimental value where experimental uncertainty was $\pm 5 \%$.

For further analysis, mean flow streamlines are superimposed with averaged surface Nusselt contours of a ribbed wall and outer wall as shown in Figure 5.2. The rib induced helical vortices form behind the rib, resulting in high local heat transfer near the origin of the vortex. These helical vortices with the secondary flow motion cause flow impingement on the outer wall, which increases heat transfer. However, in the wall modeled calculation, the vortex structure emanating from the top rib does not result in the same level of augmentation as the bottom rib. This contrary to that found in the wallresolved LES calculations as seen in Figure 5.2 (b). Figure 5.3 shows the secondary flow motions in a plane parallel to the rib at half pitch between ribs.

Velocity profiles were analyzed to observe the accuracy of WMLES where each velocity was normalized by a mean velocity, $U_{\text {mean }}$. As seen in Figure 5.4 (a), the streamwise velocity profile differs considerably from experiments and LES and is biased 
toward the bottom wall. Figure 5.4 (b) and (c), which are wall-normal and spanwise velocity profiles, also indicate that the secondary flow is not well captured by the wall modeled LES.

Figure 5.5 shows contour plots of TKE at different $\mathrm{z}$ locations where the right column of the figure shows mean flow streamlines superimposed with TKE contour plots to better illustrate how helical vortices contribute to TKE values. At $\mathrm{z}=0.25$, there are 2 distinct regions where significant TKE is present: on top of the ribs and behind the ribs where the helical vortex originates. As the plane moves toward the outer wall at the bottom, the high TKE region increases in size along the direction of the helical vortex, which becomes more turbulent due to mixing. However, the top half of the geometry shows a different pattern compared to the bottom half. As the cross-sectional plane (xyplane) moves toward the outer wall, the helical vortex diffuses or breaks up and does not maintain any coherency. Consequently, TKE values are more diffused and lower than at the bottom wall.

In addition, TKE contour plots at half pitch between ribs were examined as seen in Figure 5.6 and Figure 5.7. As expected, the figures are in accordance with the direction of helical vortices and secondary flow induced by the ribs. Figure 5.6 (a) shows a close match near the bottom ribbed wall with LES results (Figure 5.6 (b)). In contrast, near the top ribbed wall, high TKE values are associated with this region, which could be the remnants of the vortex from the previous rib. As seen in Figure 5.7 (a), which is plotted behind the top side rib, the region associated with the vortex is larger with lower values of TKE. 
Trends similar to TKE are also found in the turbulent shear stress $\overline{u^{\prime} v^{\prime}}$ contour plots at $\mathrm{z}=0.25,0.50$, and 0.75 as seen in Figure 5.8. While the distributions are quite similar at the origin of the vortex on the top and bottom walls, the top vortex structure diffuses or breaks up as it moves toward the outer wall.

Heat transfer augmentation ratios of the bottom ribbed wall and outer wall are observed in Figure 5.9. The rib induced helical vortices behind the rib enhances heat transfer and is within $7 \%$ of experimental data by Chanteloup et al. [33]. The overall heat transfer contours indicate good agreement on the bottom ribbed wall whereas underprediction of heat transfer on the outer wall is seen near the vicinity of the top rib. This is in contrast to the experimental and LES results, which are symmetric about the centerline. The extent and level of heat transfer augmentation is overpredicted on the outer wall in the region of impingement at the bottom rib.

The inner wall heat transfer augmentation ratios are analyzed against LES results by Abdel-Wahab [25] as seen in Figure 5.10. The shear layer formation on top of ribs enhances heat transfer. WMLES results, Figure 5.10 (a), shows slight overprediction compared to LES results in Figure 5.10 (b). However, unlike LES results where heat transfer is significantly enhanced by as much as $80 \%$ at the center, WMLES results show a maximum of $68 \%$ increase in heat transfer.

\subsection{Conclusions}

WMLES of fully developed $45^{\circ}$ angled ribbed duct is presented with heat transfer and compared against computational and experimental data at a bulk Reynolds number of approximately 50,000. LES with the near wall treatment reduced computational resources 
by a factor of 4 compared to traditional LES. One of the major factors which affected the predictions was the large asymmetry observed in the secondary flow between the top and bottom ribbed walls. Similar to the orthogonal ribbed duct cases, approximately 5\% difference was observed with calculations without wall modeling. Despite these differences, TKE and $\overline{u^{\prime} v^{\prime}}$ contour plots were within 9\% of LES. Surface-averaged heat transfer augmentation results were within $7 \%$ of experimental data, and the overall heat transfer augmentation ratio was within $6 \%$ difference. 


\subsection{Tables}

Table 5.1 The summary of ribbed duct calculation with and without wall treatment against wall resolved LES by Abdel-Wahab [25] and experimental data by Chanteloup et al. [33].

\begin{tabular}{|c|c|c|c|c|}
\hline & $\begin{array}{l}\text { With Wall } \\
\text { Treatment }\end{array}$ & $\begin{array}{c}\text { Without Wall } \\
\text { Treatment }\end{array}$ & $\begin{array}{c}\text { WRLES } \\
\text { Abdel-Wahab } \\
{[25]}\end{array}$ & $\begin{array}{c}\text { Experiment } \\
\text { Chanteloup et al } \\
\text { [33] }\end{array}$ \\
\hline $\begin{array}{c}\text { Mesh Size } \\
(\mathrm{x}, \mathrm{y}, \mathrm{z})\end{array}$ & $100 \times 80 \times 80$ & $100 \times 80 \times 80$ & $160 \times 128 \times 128$ & Not applicable \\
\hline$R e_{\tau}$ & 18,610 & 18,610 & Not applicable & Not applicable \\
\hline$R e_{b}$ & 49,562 & 49,419 & 47,304 & 50,000 \\
\hline $\begin{array}{l}\text { Form Drag } \\
\text { Loss }(\%)\end{array}$ & 86 & 85 & Not applicable & Not applicable \\
\hline \multicolumn{5}{|c|}{$\overline{N u} / N u_{0} \quad\left(N u_{0}=0.023 \cdot R e_{b}^{0.8} \cdot \operatorname{Pr}^{0.4}\right)$} \\
\hline Ribs & 3.02 & 3.14 & 3.44 & Not applicable \\
\hline $\begin{array}{l}\text { Ribbed } \\
\text { Walls }\end{array}$ & 2.52 & 2.63 & 2.37 & 2.78 \\
\hline Inner Wall & 2.03 & 2.25 & 1.88 & 1.65 \\
\hline Outer Wall & 2.70 & 2.74 & 2.55 & 2.23 \\
\hline Overall & 2.49 & 2.61 & 2.29 & 2.34 \\
\hline \multicolumn{5}{|c|}{$f / f_{0} \quad\left(f_{0}=0.046 \cdot R e_{b}^{-0.2}\right)$} \\
\hline Overall & 13.32 & 13.39 & 13.80 & 12.49 \\
\hline
\end{tabular}




\subsection{Figures}

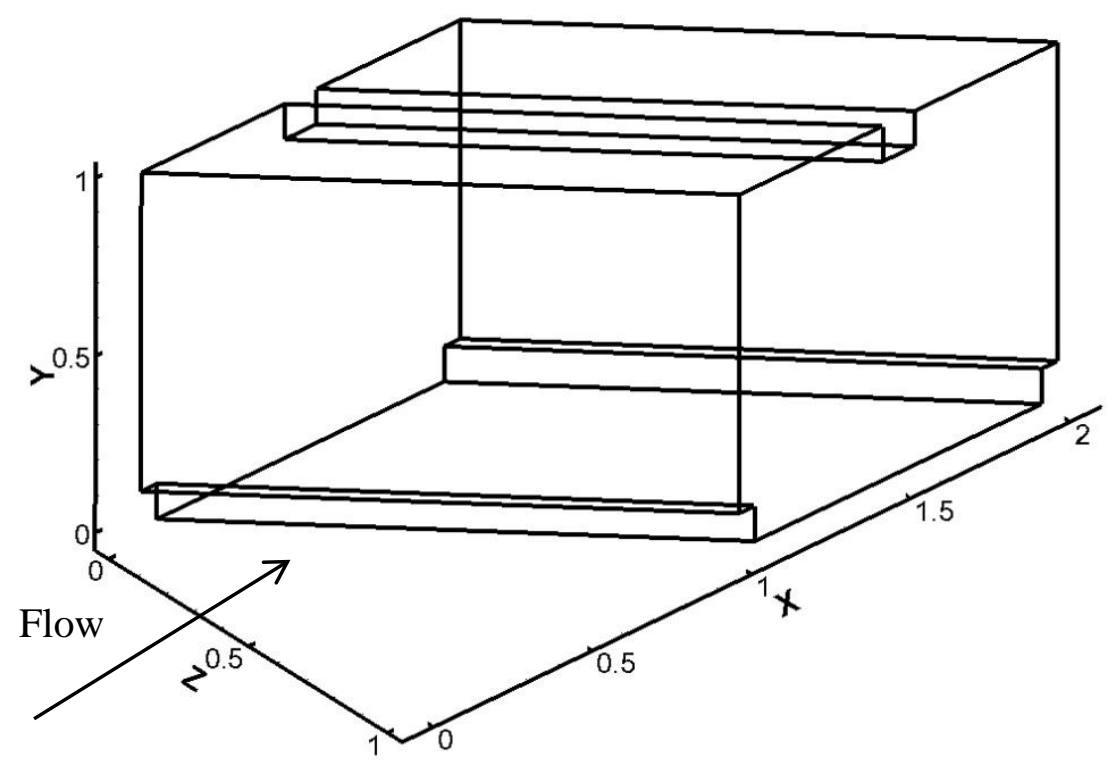

Figure 5.1 The computational domain for $45^{\circ}$ ribbed duct.

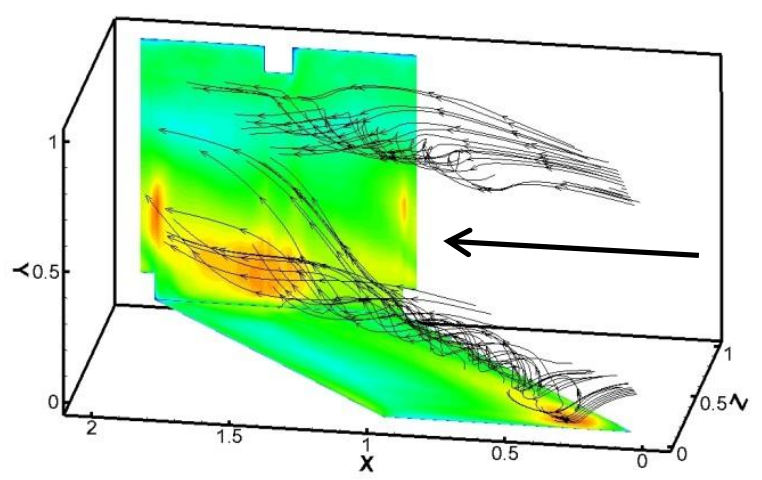

(a)

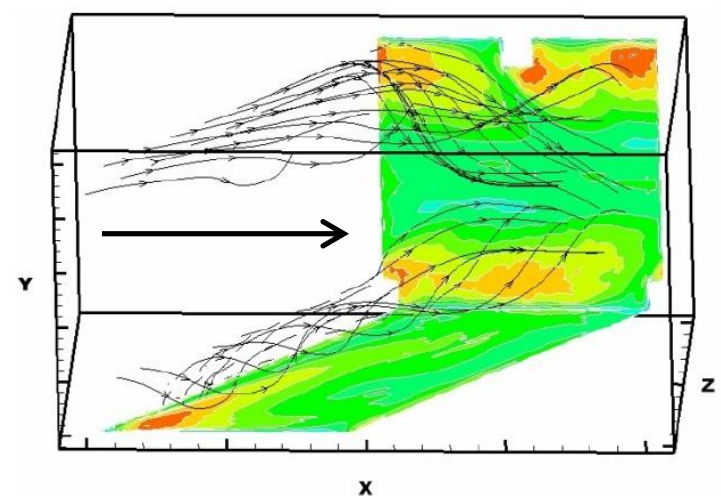

(b)

Figure 5.2 Mean flow streamlines superimposed on the average surface Nusselt number contours: (a) WMLES at $\mathrm{Re}=\mathbf{4 9 , 0 0 0}$ where flow moves from right to left (b) LES by Abdel-Wahab [25] at $\operatorname{Re}=47,000$ where flow moves from left to right. 


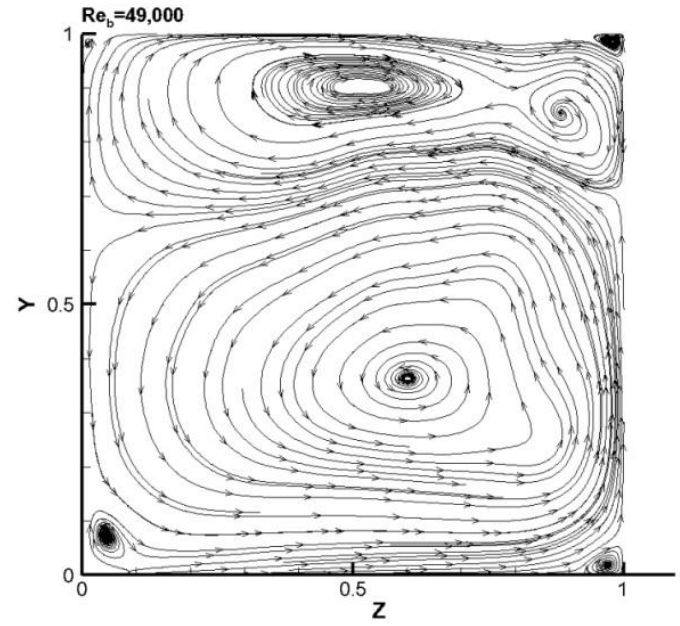

(a)

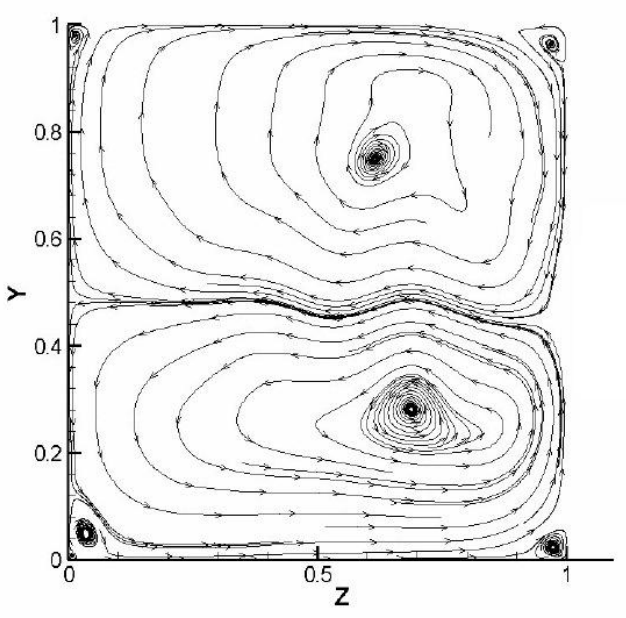

(b)

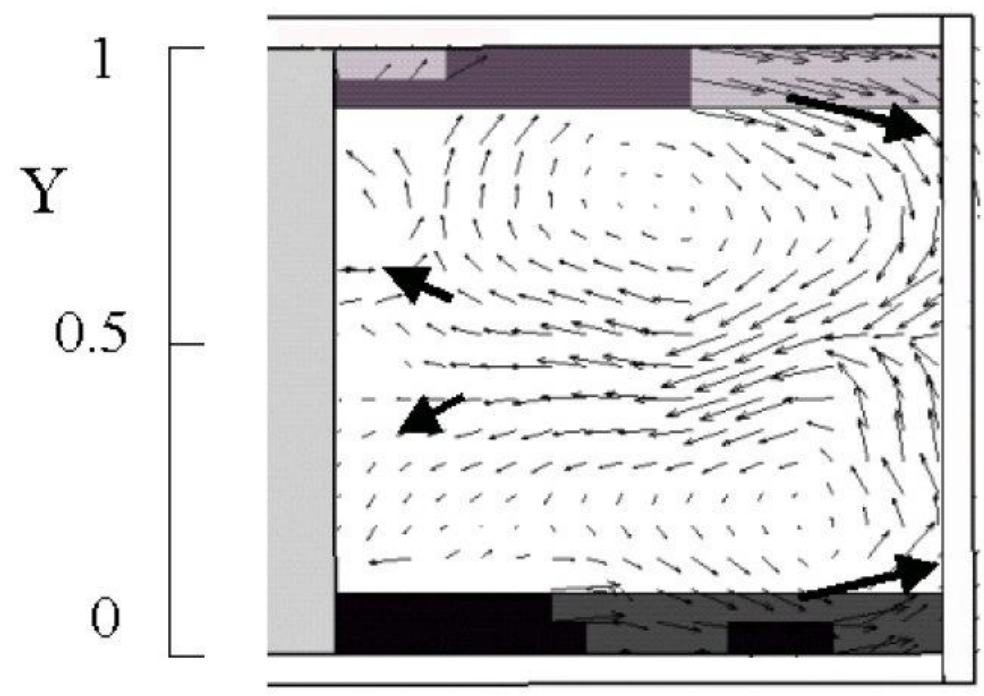

(c)

Figure 5.3 Mean secondary flow in a plane parallel to ribs at a half pitch: (a) WMLES at $\operatorname{Re}=49,000$ (b) LES by Abdel-Wahab [25] at $\operatorname{Re}=47,000$ (c) Chanteloup et al. [33] at $\operatorname{Re}=50,000$. 


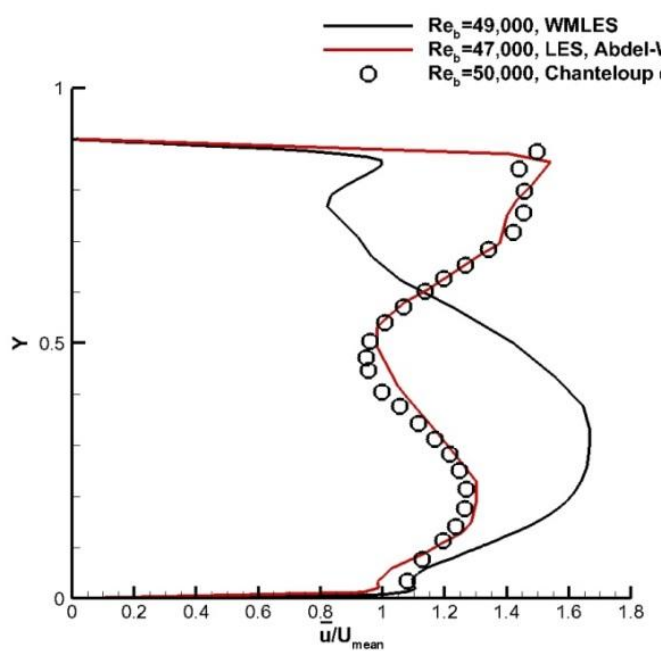

(a)

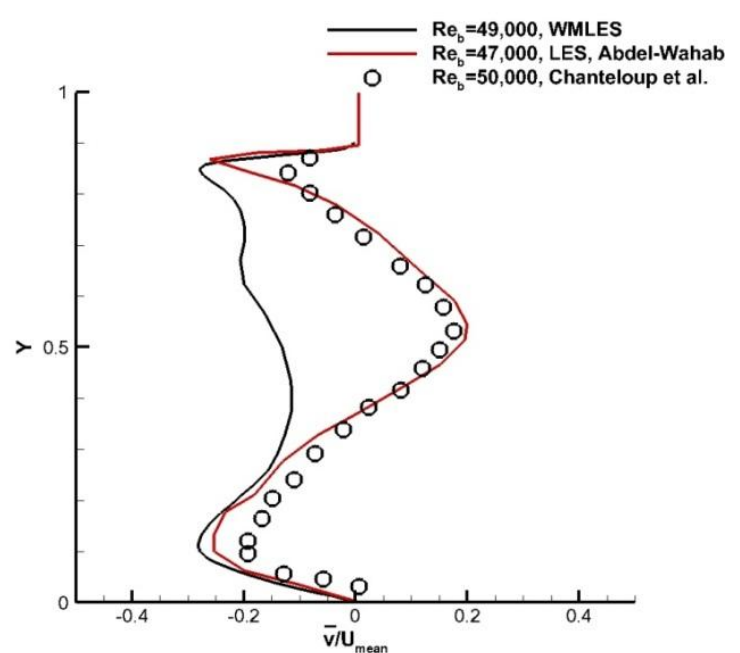

(b)

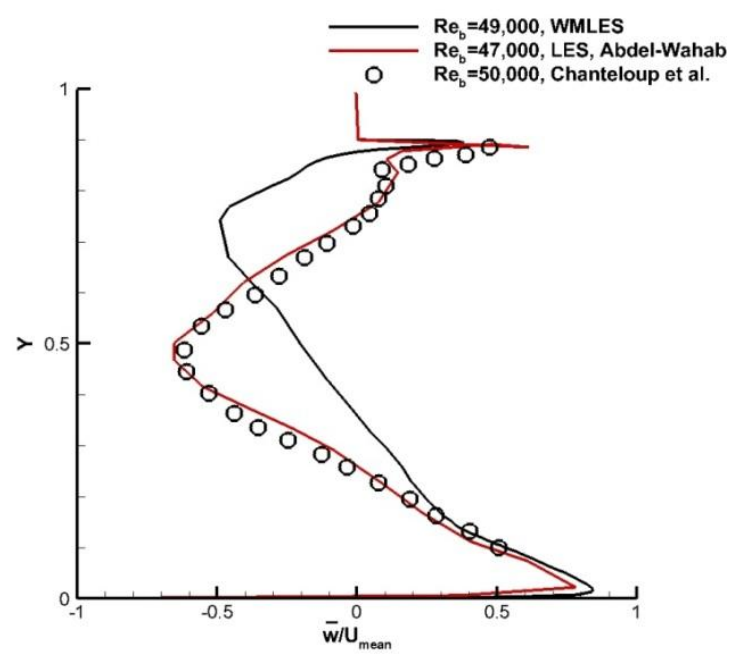

(c)

Figure 5.4 Comparison plot of averaged mean velocity profiles in the center of the duct in a plane cutting through the middle of a top rib $(x=1, z=0.5)$ against LES and experimental data: (a) streamwise velocity profile (b) wall normal velocity profile (c) spanwise velocity profile. 

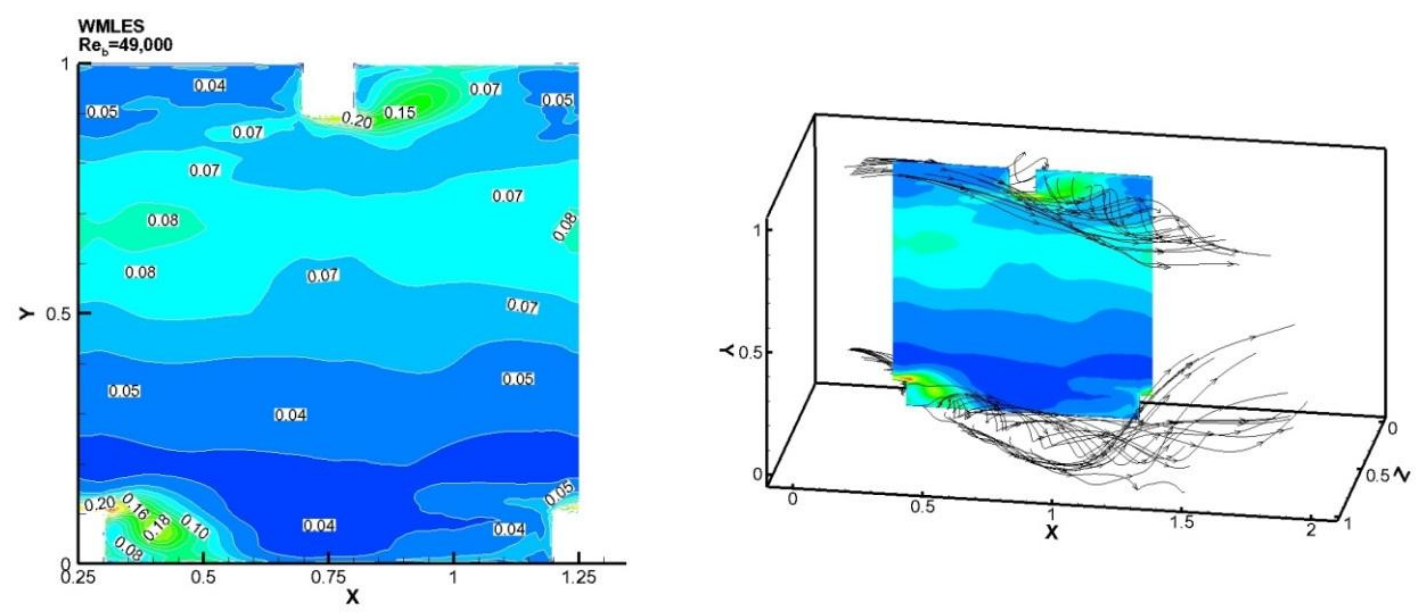

(a)
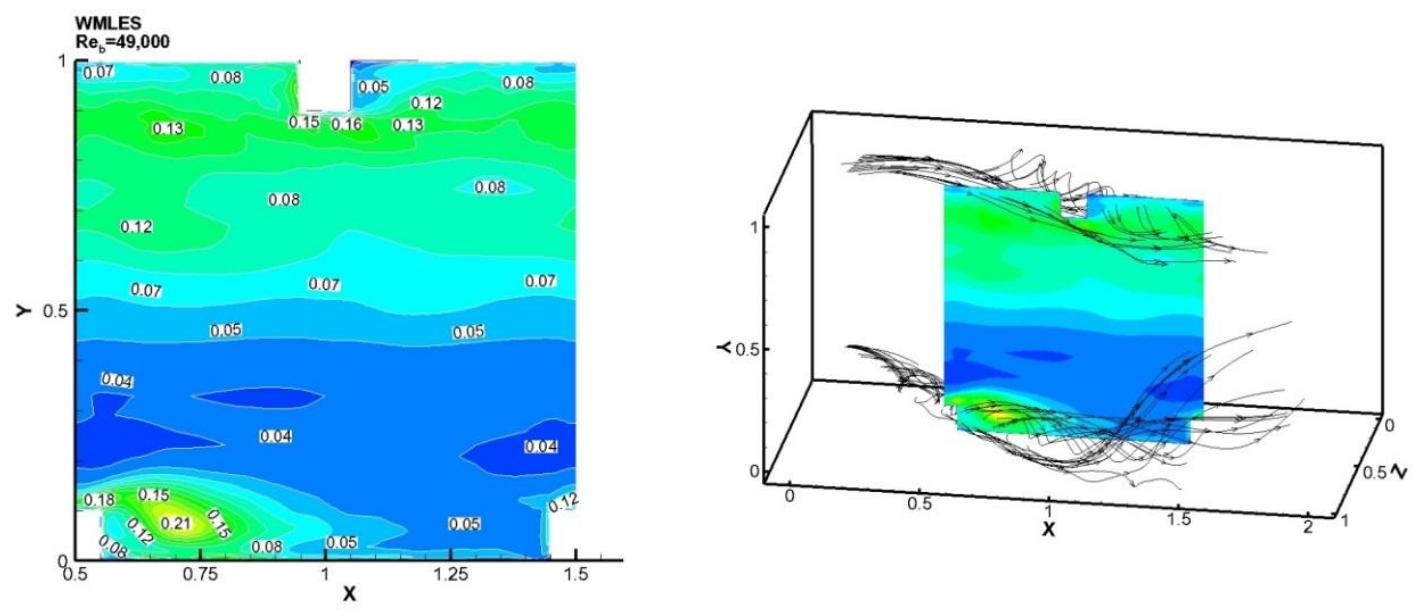

(b)
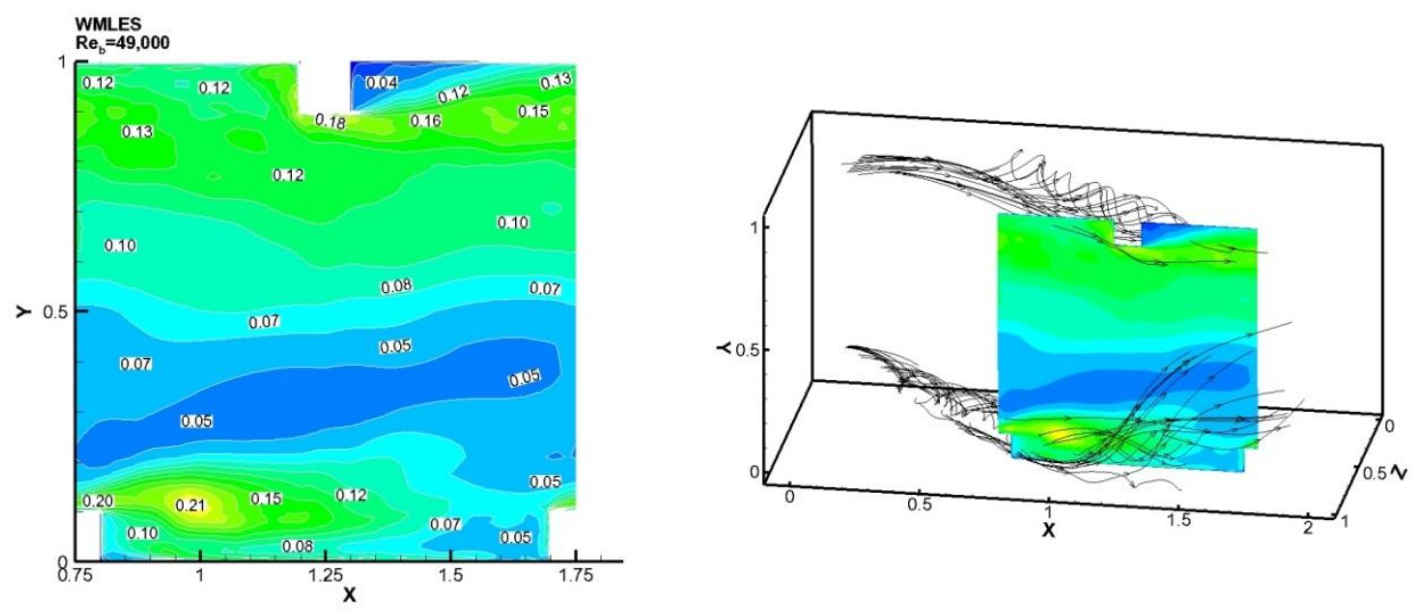

(c)

Figure 5.5 Contour plots of turbulent kinetic energy (TKE) and mean flow streamlines at different spanwise locations: (a) $z=0.25$ (b) $z=0.50$ (c) $z=0.75$. 

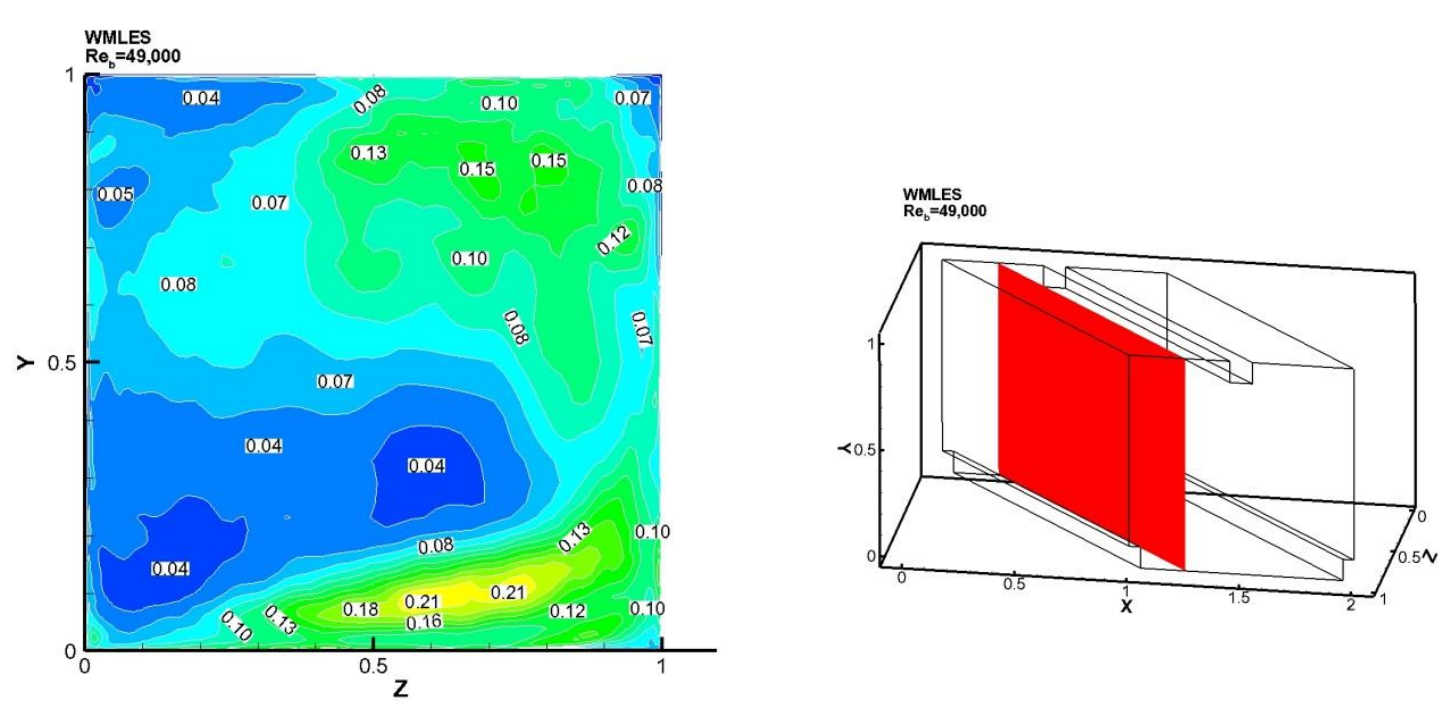

(a)
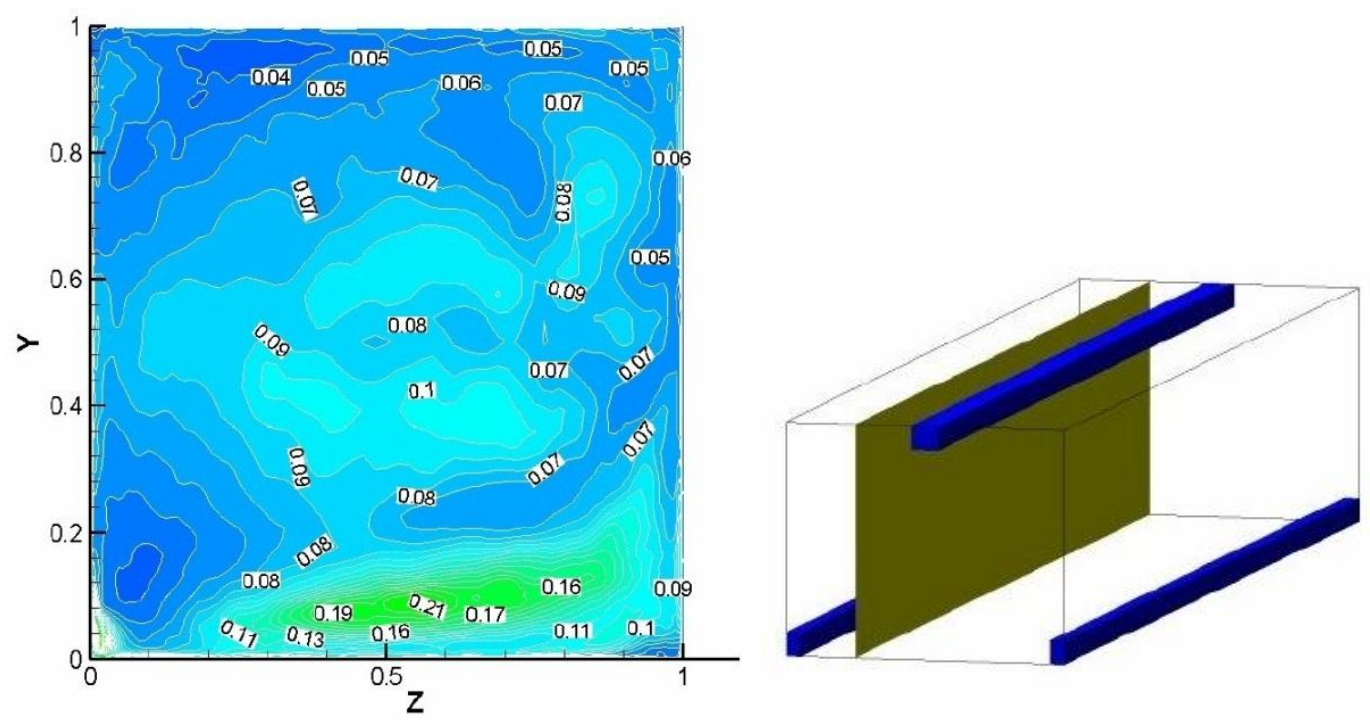

(b)

Figure 5.6 Turbulent kinetic energy (TKE) at a plane in parallel to ribs (half pitch infront of the top rib): (a) WMLES at $\operatorname{Re}=49,000$ (b) LES by Abdel-Wahab [25] at $\operatorname{Re}=47,000$. 

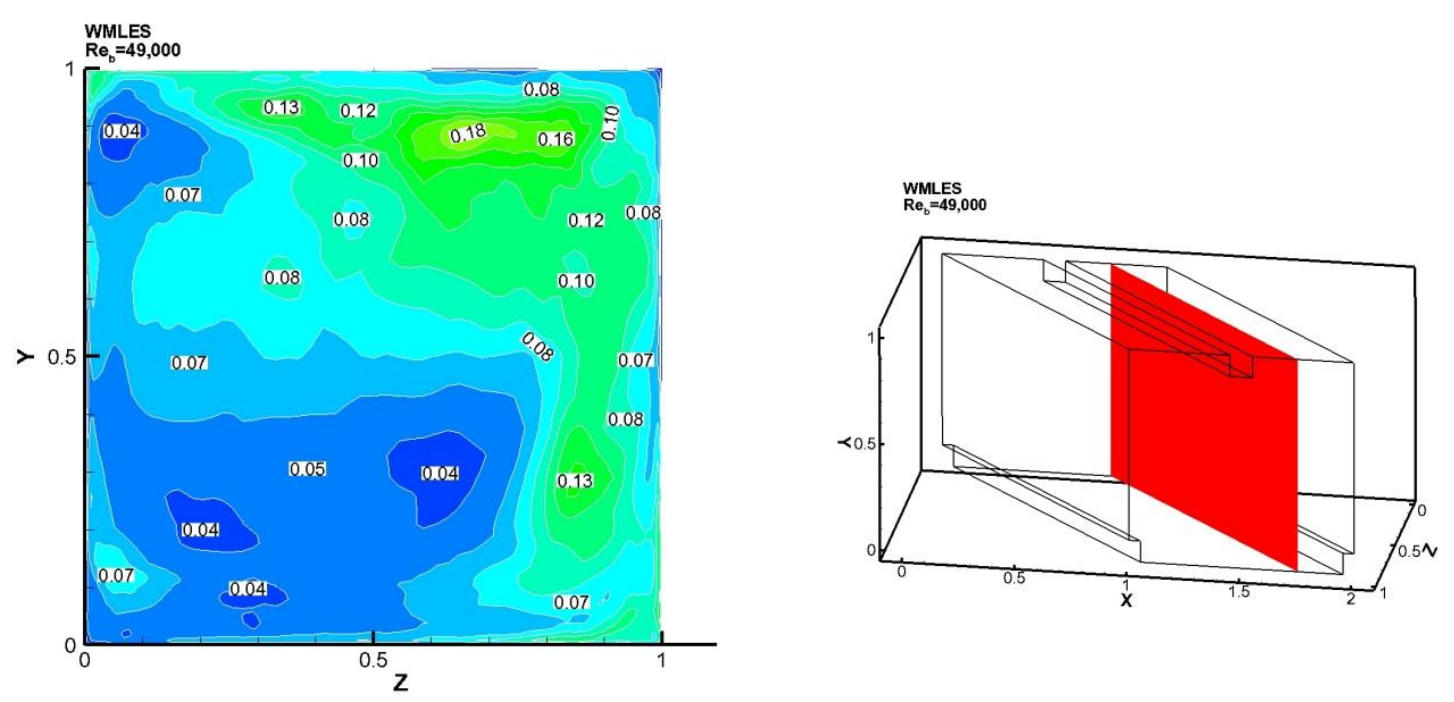

(a)
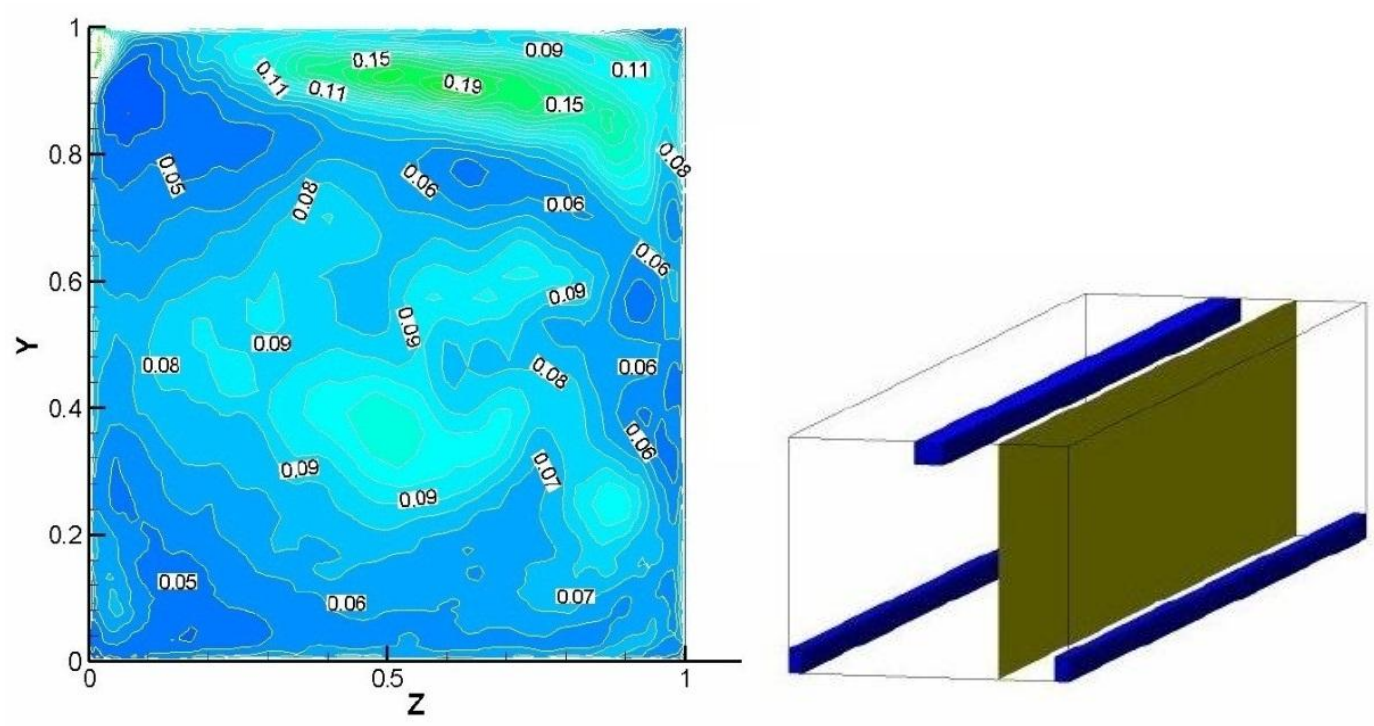

(b)

Figure 5.7 Turbulent kinetic energy (TKE) at a plane in parallel to ribs (half pitch behind of the top rib): (a) WMLES at $\operatorname{Re}=49,000$ (b) LES by Abdel-Wahab [25] at $\operatorname{Re}=47,000$. 


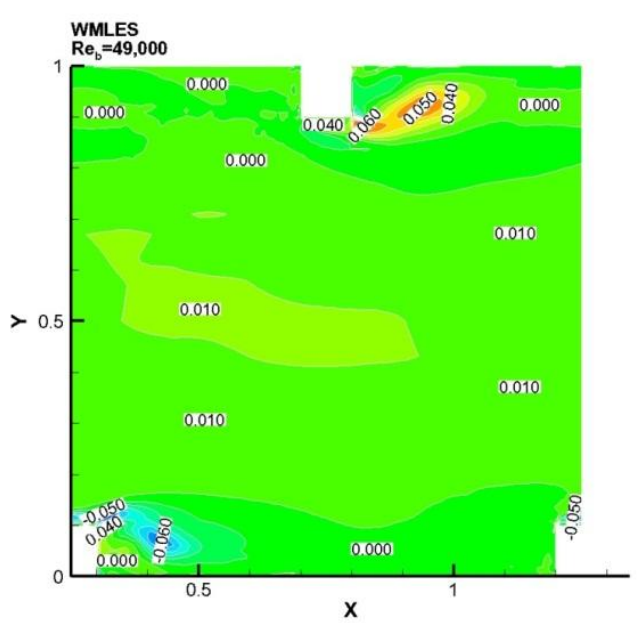

(a)

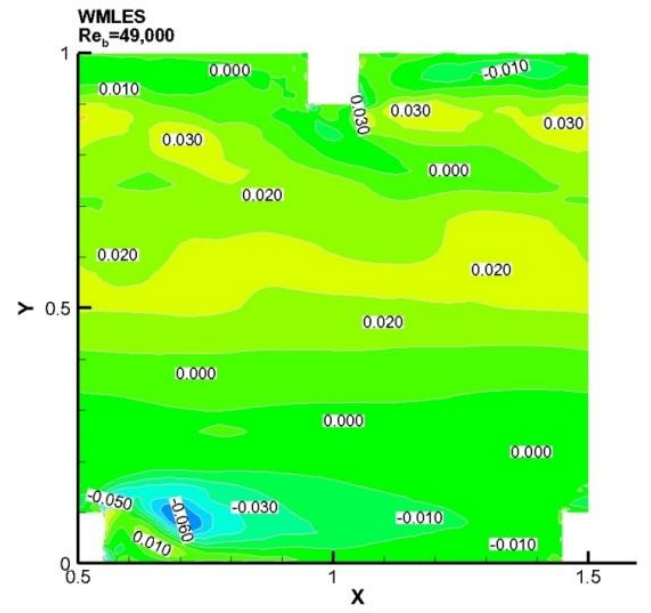

(b)

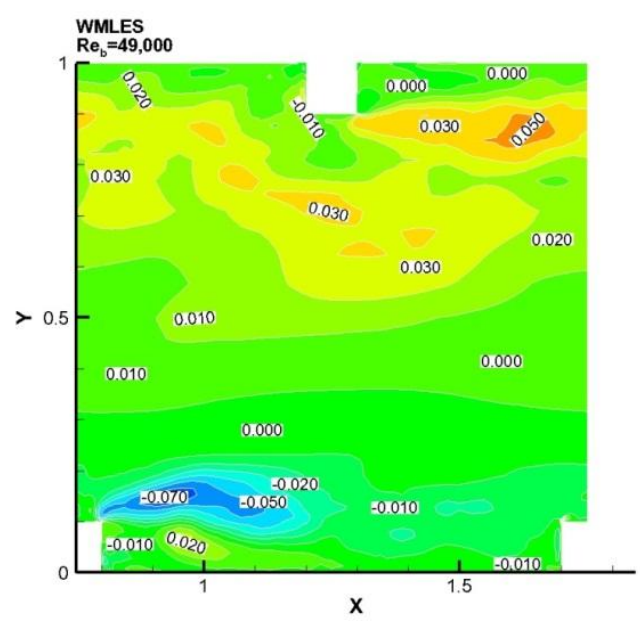

(c)

Figure 5.8 Mean turbulent shear stress, $\overline{u^{\prime} v^{\prime}}$, at different spanwise locations: (a) $z=$ 0.25 (b) $z=0.50$ (c) $z=0.75$. 


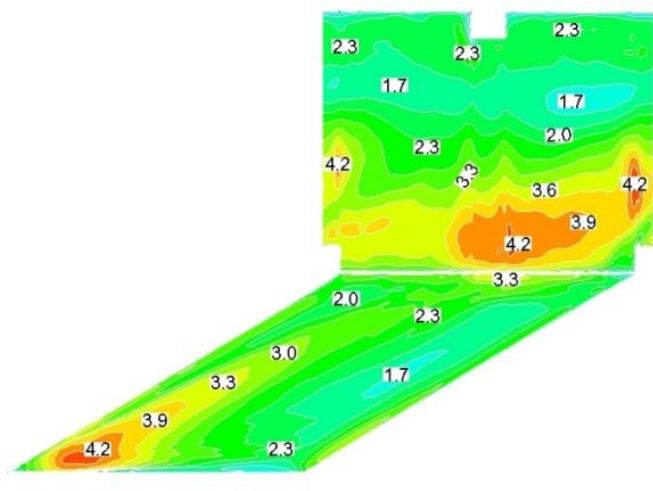

(a)

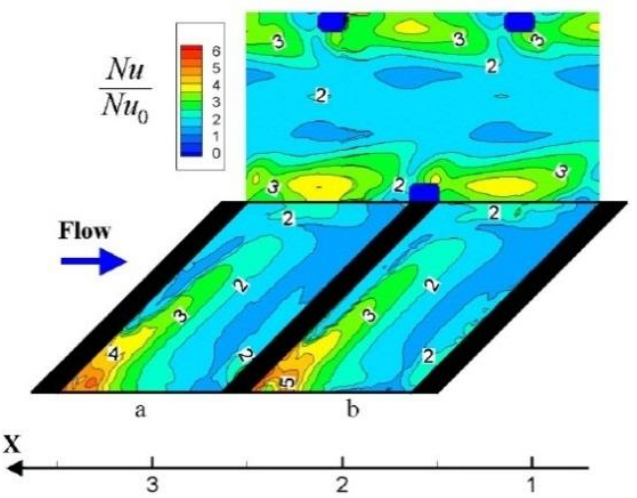

(b)

Figure 5.9 Averaged heat transfer augmentation ratios on a ribbed wall and outer wall: (a) WMLES at $\operatorname{Re}=49,000$ (b) Chanteloup et al. [33] at $\operatorname{Re}=\mathbf{5 0 , 0 0 0 \text { . }}$

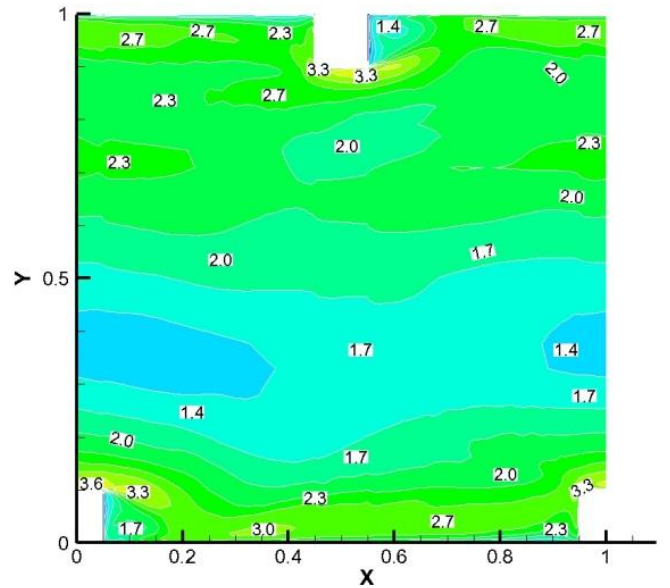

(a)

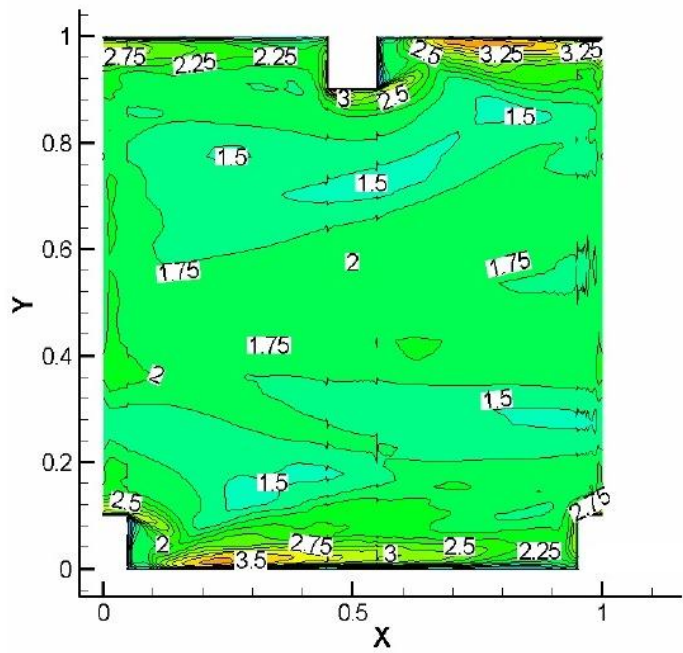

(b)

Figure 5.10 Averaged heat transfer augmentation ratios on a inner wall: (a) WMLES at $\operatorname{Re}=49,000$ (b) Abdel-Wahab [25] at $\operatorname{Re}=47,000$. 


\section{Chapter 6 Wall Modeled Large-Eddy Simulations of Two-pass Smooth Duct with U-Bend, Heat Transfer, Rotation, and Centrifugal Buoyancy}

\subsection{Introduction}

In this chapter the wall model is tested in a developing two-pass smooth duct with a U-bend. Because of the developing nature of the turbulent flow, the synthetic eddy method (SEM) is used to generate inlet conditions. In addition to the model evaluation in previous chapters, these set of calculations test the performance of the wall model in predicting very subtle secondary motions set up in a smooth square duct, its use with property variations which have a large impact on heat transfer, and use in strong streamline curvature and pressure gradients as they exist in the U-bend geometry.

Three main sets of primary calculations are performed based on the experiments of Wagner et al. [38] on a two-pass duct of square cross-section with U-bend: the stationary geometry at a bulk Reynolds number of 25,000 , with rotation at a bulk rotation number $\left(R o_{b}=0.238\right)$ at two density ratios of 0 and 0.13 . To generate the mean and turbulent statistics at the inlet, which are used as inputs to the SEM, an auxiliary simulation for fully-developed duct flow is also performed. For flow analyses, mean velocity and turbulent profiles are compared against the LES data by Madabhushi and Vanka [39], and experimental data of Liou and Chen [40], and Liou et al. [41]. Heat transfer predictions are compared to the experiments of Wagner et al. [38].

The effect of Coriolis forces on the first and second pass was clearly seen in WMLES results due to the change in the direction of the flow. While the effect of the 
density ratio indicated a slight decrease in heat transfer values for stationary simulations, heat transfer values of rotational simulations were enhanced with a significant increase within the bend. Despite the uncertainty of the bend geometry (Wagner et al. [38]), results indicated favorable comparisons based on flow and heat transfer analyses. It is also found that the bend geometry appears to significantly affect both flow and heat transfer values.

\subsection{Literature Review}

The primary investigation was done based on the experimental data by Wagner et al. [38], which consisted of a series experiments at bulk Reynolds number of 25,000 with bulk rotation numbers of $0,0.118,0.238,0.350$, and 0.475 conducted at density ratios of $0.07,0.13,0.18$, and 0.22 with constant temperature boundary conditions. The inlet pressure and temperature were set to approximately 10 atmospheres and $300 \mathrm{~K}$, respectively, at a mean radius from the axis of rotation of $\bar{R} / D=49$. Measurements were done on two-passes including a U-bend for the stationary and 0.238 bulk rotation cases with a density ratio of 0.13 . With an increase in density ratios, heat transfer values in the first pass were more responsive to the change in density ratio than the second pass.

Overall, with an increase in bulk rotation numbers, the first pass, outward flow, leading side heat transfer augmentation ratio values decrease until approximately 0.2 bulk rotation number. In contrast, the trailing side heat transfer values consistently increase with an increase in bulk rotation numbers for the first pass. For the second pass, inward flow, the leading side showed higher heat transfer values than the trailing side due to the change in direction of Coriolis forces. The outer side wall heat transfer values indicated 
that the local heat transfer value was the highest at the center of the bend. The inner wall heat transfer values were not measured within the bend but only for the first and second passes.

Han et al. [32] did an experimental heat transfer study on a rotating two-pass square channel with smooth walls. Bulk Reynolds numbers were varied from 25,000 to 2,500 for bulk rotation numbers from 0.0352 to 0.352 with a mean radius ratio of 30 . For thermal boundary conditions, constant wall temperatures on 4 walls, constant heat fluxes on 4 walls, and trailing wall temperature hotter than leading wall with side walls insulated were conducted with density ratios below 0.1 . For a bulk rotation number of 0.352 and a density ratio of 0.1 with 4 walls at constant temperatures, the first pass leading side indicated an decrease in heat transfer augmentation ratios, which then increase downstream, whereas a consistent increase in heat transfer augmentation ratios was observed on the first pass trailing side. The second pass heat transfer data indicated a reversal effect. The overall difference between the leading and trailing sides' heat transfer augmentation ratios decreased on the second pass relative to the first pass heat transfer data. In contrast, with uneven wall temperature boundary conditions, the overall heat transfer augmentation ratios of all 4 sides indicated higher values compared to fixed temperature and flux boundary conditions. Han et al. [32] noted that the results are due to the secondary flow motion, which tends to drive the flow at higher rotation rates.

Johnson and Launder [42] conducted an experimental study on a heated squaresectioned duct with U-bend at a bulk Reynolds number of approximately 56,000 . The ratio of a mean bend radius to hydraulic diameter of the duct was 3.35:1 with uniform heat flux boundary conditions. Detailed observations were made on streamwise velocity 
profiles, $u_{r m s}$ profiles, and local Nusselt number variations within the two-passes and Ubend. The streamwise velocity profile indicated that due to flow acceleration near the inner wall and flow deceleration near the outer wall causes a velocity bias towards the inner wall early in the bend. However, in the mid-bend location, the secondary flow motion shifts the local velocity profile toward the outer wall. As the flow moves around the bend and downstream, the velocity profile is biased towards the outer wall. Similarly, the $u_{r m s}$ profiles indicate slightly biased behavior towards the inner wall past the midbend location. However, the $u_{r m s}$ values at the outer wall recover downstream. In addition, the outer wall to inner wall Nusselt number ratio exceeds by $3: 1$ compared to approximately 2:1 in Seban and McLaughlin [43] where a curved circular pipe with a mean bend radius to hydraulic diameter ratio of 104 with bulk Reynolds numbers from 6,000 to 65,600 for turbulent flow was used. The expectation of Johnson and Launder [42] was to obtain higher Nusselt number ratios compared to Seban and McLaughlin [43] due to a mean bend radius to hydraulic diameter ratio difference, which was not evident in the study. For further analyses, Johnson and Launder [42] recommended a low Reynolds number study on mean and turbulent quantities within the bend.

Iacovides et al. [44] did an experimental study on a rotating two-pass with a square-ended U-bend. The flow analyses was done at a bulk Reynolds number of 100,000 whereas a bulk Reynolds number of 30,000 was used for heat transfer analysis at a bulk rotation number of 0.2 . The bend inner radius to the duct hydraulic diameter ratio was 0.15 with Prandtl number of 5.9. The flow analyses indicated that due to a change in Coriolis forces in the second pass, the mean velocity vectors were biased toward the leading side, which translated to a heat transfer enhancement. Also, the heat transfer data 
showed that Nusselt values were enhanced by approximately 2 times relative to the fullydeveloped heat transfer value.

Liou and Chen [40] investigated a two-pass square smooth duct with bulk rotation numbers of 0 and 0.082 . Flow analyses were primarily done with a laser Doppler velocimetry (LDV) to measure velocity and turbulent profiles at a bulk Reynolds number of 14,000 . The sharp $180^{\circ}$ bend caused disturbances on the main flow up to 3 hydraulic diameter, $D_{h}$, before the bend on the first pass and $11.2 D_{h}$ downstream of the second pass. Liou and Chen [40] further evaluated the flow by examining velocity and turbulent profiles along with secondary flow motions within the bend. However, due to a difference in the bend geometries, only fully-developed velocity and turbulent profiles were extracted for comparisons in this chapter.

In addition, Liou et al. [41] observed the effects of different bulk rotation numbers from 0 to 0.2 at a bulk Reynolds number of 10,000 . LDV was used to measure velocity and turbulent profiles where heat transfer analysis was also done in this study. The analyses were done similar to Liou and Chen [40] with emphasis on velocity profiles, turbulent profiles, and secondary flow motion at a higher bulk Reynolds number of up to 0.2. One of interesting observations was that with rotation, the averaged and maximum magnitudes of secondary flow motion increased linearly with the bulk rotation number. Both secondary flow magnitudes and heat transfer augmentation ratios indicated higher rate of increase on the leading side than the trailing side as bulk rotation numbers increase. Furthermore, heat transfer and turbulent kinetic energy were enhanced by as much as $40 \%$ relative to the stationary data. 
Madabhushi and Vanka [39] did a computational study on a square duct by using the LES method. A bulk Reynolds number of 5,810 in $65 \times 65 \times 32$ grid size was used with a wall-normal and spanwise wall coordinates of less than 10 and a streamwise wall coordinate of approximately 70 . The results indicated that the secondary fluid motion exhibited 8-way symmetry about the diagonal axes. Predicted mean and turbulent profiles were in reasonable agreement with experiments.

Patil [2] simulated a developing turbulent channel flow at a shear velocity based Reynolds number, $R e_{\tau}$, of 395 with WMLES and the synthetic eddy method (SEM). Unlike the presented study in this chapter, a spatially repeating domain in spanwise direction was applied to the domain. The computational domain size was $12 \pi \delta \times 2 \delta \times$ $\pi \delta$, which consisted of $768 \times 96 \times 128$ in streamwise, wall-normal, and spanwise directions. For the SEM, the DNS results of Kim et al. [18] at $R e_{\tau}$ of 590 were used to generate 1,000 randomly placed artificial eddies to generate the inlet turbulence. Timeaveraged streamwise velocity and Reynolds stress profiles were compared at different streamwise location to observe the accuracy of the SEM. Patil [2] observed that the friction coefficient initially drops by approximately $3 \%$ but regains to the correct value after $10 \delta$, or 10 channel half widths. Similar observations were made on Reynolds stresses profiles, which indicated that the SEM accurately modeled the inlet turbulence with reference to the DNS results by Kim et al. [18].

\subsection{Computational Model and Details of Fully-developed Smooth Duct Flow}

The computational model uses the fully-developed model mentioned in Chapter 2.3. The governing flow equations are non-dimensionalized by a characteristic length 
scale, which is a hydraulic diameter, $D_{h}$, and a characteristic velocity scale given by the friction velocity, $u_{\tau}=\sqrt{\Delta \bar{P}_{x} / \rho}$. The assumed periodicity of the domain in the streamwise or $\mathrm{x}$-direction requires pressure and temperature to be decomposed as mentioned in Chapter 2.

At a nominal bulk Reynolds number, $R e_{b}$, of 25,000 , the stationary simulation is examined for the SEM input. $R e_{b}$ is defined as follow:

$R e_{b}=\frac{\bar{u}_{b} D_{h}}{v}$

where $\bar{u}_{b}$ is the time-averaged bulk velocity, $D_{h}$ is a hydraulic diameter, and $v$ is the kinematic viscosity of the medium, which is air.

The computational domain for the smooth duct simulations is presented in Figure 6.1. The computational domain consists of approximately $5.5 \times 10^{4}$ cells, or $54 \times 32 \times$ 32 cells in $i, j$, and $k$ directions where $i$ is the streamwise direction, $j$ is the vertical wallnormal direction, and $\mathrm{k}$ is the spanwise direction. A uniform grid distribution is used in $\mathrm{x}$ direction with a spacing value of approximately 0.07 , which is equivalent to $\Delta x_{1}{ }^{+} \sim 98$. A non-uniform grid distribution is used in $\mathrm{y}$ and $\mathrm{z}$ directions with spacing values of 0.03 or a $\Delta z^{+}$value of 41 . These values were based on the specified $R e_{\tau}$, which is approximately 2,758. Wall boundary conditions are set on both xy- and xz-planes whereas periodic boundary conditions are set on yz-boundary planes.

The non-dimensional time step value of $5 \times 10^{-4}$ is used and time averaged for 10 non-dimensional time units after a stationary state is reached. The momentum and pressure equations are solved implicitly to the convergence criteria value of $1 \times 10^{-6}$ and $1 \times 10^{-6}$ at each time step, respectively. The fully-developed smooth duct cases utilize 1 
core of an Intel Xeon Linux cluster. Each time step took approximately $15.0 \mu$ s of wall clock time/grid node.

All quantities are non-dimensionalized by $u_{b}$ or $u_{b}^{2}$ unless specified otherwise. Also, Reynolds number is equivalent to the bulk Reynolds number, $R e_{b}$.

\subsection{Computational Model and Details of Developing Smooth Duct with U-bend}

The computational model assumes incompressible variable property flow with the SEM described in Chapter 2.5. The developing smooth duct with U-bend consists of 4 smooth walls, which experiences a positive z-direction rotation at an angular velocity, $w_{z}$ in $\mathrm{rad} / \mathrm{s}$. The governing flow equations are non-dimensionalized by a characteristic length scale, which is a hydraulic diameter, $D_{h}$, and a characteristic velocity scale given by the bulk velocity, $u_{b}$.

At a nominal bulk Reynolds number, $R e_{b}$, of 25,000, the smooth developing duct was examined for bulk rotation numbers, $R o_{b}$, of 0 and 0.238 with density ratios of 0 and 0.13. $R e_{b}$ and $R o_{b}$ are defined as follow:

$R e_{b}=\frac{\bar{u}_{b} D_{h}}{v}$

$R o_{b}=\frac{w_{z} D_{h}}{\bar{u}_{b}}$

where $\bar{u}_{b}$ is the time-averaged bulk velocity, $D_{h}$ is a hydraulic diameter, $v$ is the kinematic viscosity of the medium, which is air for all simulations, and $w_{z}$ is the angular velocity in z-axis.

The computational domain for the developing smooth duct simulations is presented in Figure 6.2 (a), which is based on the experimental apparatus from Wagner et 
al. [38]. While the first and second pass geometry is well defined, the bend geometry is not well defined in the paper. We have reproduced the bend geometry as closely as we could based on visual observations of Fig. 1(b) in Wagner et al. [38]. The nominal bend ratio (the mean bend radius to hydraulic diameter of the duct) is kept at approximately $1.55: 1$. The approximated bend geometry introduces an increase in cross-sectional area at the beginning of the bend followed by a converging section at the center of the bend. Unfortunately, the geometry will have an impact on the flow and heat transfer observed in the bend, and the geometric uncertainty will prevent an exact one-to-one comparison with the experiments.

For the presented study, two different grid sizes are used. The coarse mesh consists of approximately $6.3 \times 10^{5}$ cells, or $620 \times 32 \times 32$ cells in i, j, and k directions where $\mathrm{i}$ is the streamwise direction, $\mathrm{j}$ is the vertical wall-normal direction, and $\mathrm{k}$ is the spanwise direction. The fine mesh consists of approximately $7.4 \times 10^{5}$ cells, or $720 \times 32$ $\times 32$ cells in $\mathrm{i}, \mathrm{j}$, and $\mathrm{k}$ directions. A non-uniform grid distribution is used in $\mathrm{y}$ and $\mathrm{z}$ directions with spacing values of approximately 0.03 for both directions, which is equivalent to $\Delta y_{1}{ }^{+}$and $\Delta z_{1}{ }^{+}$values of 41 . These values were based on the specified $R e_{\tau}$, which is approximately 2,758 on average.

The difference between two grid sizes is the streamwise grid resolution within the bend. Compared to a uniform grid distribution in the first and second straight passes with a spacing value of 0.07 , which is equivalent to $\Delta x^{+}$value of 98 , a higher grid resolution is used within the bend in an attempt to better capture the flow physics. Specifically in the bend, $\Delta x_{1}{ }^{+}$for the coarse grid is 36 , or a spacing value of 0.026 , and $\Delta x_{1}{ }^{+}$for the fine grid is 18 , or a spacing value of 0.013 . 
Both the inlet and exit lengths were increased by approximately $3 D_{h}$ in the computational domain to have smooth transitions at the inlet and exit. However, the constant temperature boundary condition was applied only within the region of interest, which excluded the first $3 D_{h}$ from the inlet. These constant temperature wall boundary conditions are set on all 4 walls: inner, outer, leading, and trailing walls. The ratio of the mean bend radius to hydraulic diameter of the duct is approximately 1.55:1 due to a flat part of the bend on the outer wall ( $z=1.525$ to 2.05 ), resulting in a diverging-converging cross-section. Furthermore, based on the geometry shown in Wagner et al. [38], the equivalent geometry in this simulation is from $x=3.1$ first pass to $x=3.1$ second pass, which theoretically should attach to the second U-bend leading to the third pass, which is not part of the study.

For the coarse mesh, the non-dimensional time step value of $5 \times 10^{-3}$ is used and time averaged for 50 non-dimensional time units. The momentum and pressure equations are solved implicitly to the convergence criteria value of $1 \times 10^{-6}$ and $4 \times 10^{-5}$ at each time step, respectively. All smooth duct with U-bend cases utilize 4 cores of an Intel Xeon Linux cluster. Each time step takes approximately $11.2 \mu$ s of wall clock time/grid node.

For the fine mesh, the non-dimensional time step value of $2 \times 10^{-3}$ is used and time averaged for 50 non-dimensional time units. The momentum and pressure equations are solved implicitly to the convergence criteria value of $1 \times 10^{-6}$ and $4 \times 10^{-5}$ at each time step, respectively. All smooth duct with U-bend cases utilize 4 cores of an Intel Xeon Linux cluster. Each time step takes approximately $8.0 \mu$ s of wall clock time/grid node. 
For the heat transfer analysis, Nusselt number is used where the local Nusselt number is defined as:

$N u=\frac{1}{\theta_{s}-\theta_{\text {ref }}}$

where $\theta_{s}$ is the surface temperature and $\theta_{r e f}$ is the reference temperature. The averaged Nusselt number is calculated based on the local Nusselt number:

$\overline{N u}=\frac{1}{\iint_{\Omega} d S}\left[\iint_{\Omega} \frac{1}{\theta_{s}-\theta_{\text {ref }}} d S\right]$

where $S$ is the appropriate surface for the averaged Nusselt number. In addition, the averaged Nusselt number is normalized by the Dittus-Boelter equation:

$N u_{0}=0.023 \cdot R e_{b}^{0.8} \cdot \operatorname{Pr}^{0.4}$

All quantities are non-dimensionalized by $u_{b}$ or $u_{b}^{2}$ unless specified otherwise. Reynolds number and rotation numbers are equivalent to bulk Reynolds number and bulk rotation number, $R e_{b}$ and $R o_{b}$, respectively. For discussion purposes, Figure 6.2 (b), which is the cross-sectional view of the inlet $\mathrm{x}$-plane, is shown to provide correct terminologies for the following sections. The leading side is equivalent to the suction side and the trailing side is equivalent to the pressure side. Inner and outer walls are side walls, where the inner wall refers to the side wall with the shortest curvature around the bend whereas the outer wall refers to the side wall with a longest curvature around the bend. The "1" denotes the cross-sectional area of the first pass and the "2" denotes the crosssectional area of the second pass.

To generate the inlet turbulence and mean flow, mean velocity profiles, turbulent profiles, and turbulent length scales are used from the fully-developed calculation. The turbulent length scales were calculated based on the following formulation: 
$l_{i, j, k}=\frac{T K E^{3 / 2}}{\epsilon}$

$T K E=\frac{1}{2} \cdot \sqrt{\overline{u^{\prime} u^{\prime}}+\overline{v^{\prime} v^{\prime}}+\overline{\overline{w^{\prime} w^{\prime}}}}$

where $l_{i, j, k}$ is the turbulent length scale in $\mathrm{i}, \mathrm{j}$, and $\mathrm{k}$ directions and TKE is the turbulent kinetic energy where each Reynolds stresses, $\overline{u^{\prime} u^{\prime}}, \overline{v^{\prime} v^{\prime}}$, and $\overline{w^{\prime} w^{\prime}}$ are normalized by an appropriate $u_{b}$ quantity. However, the calculated turbulent length scales were quite large and unphysical. As a result, these artificial eddies were not able to move smoothly downstream and caused locally high shear stresses due to high turbulent fluctuations. Therefore, after iteratively varying turbulent length scales, fixed turbulent length scales of $0.3,0.1$, and 0.1 in $\mathrm{i}, \mathrm{j}$, and $\mathrm{k}$ directions are used in the simulations. The number of eddies used were 500 and 1,000 .

\subsection{Results of Fully-developed Duct Flow at $\operatorname{Re}=\mathbf{2 5 , 0 0 0}$}

The primary purpose of the fully-developed smooth duct flow is to obtain mean flow and turbulent data for the SEM input. Despite the differences between bulk Reynolds numbers, as shown in Figure 6.3, the mean velocity profile of WMLES predicts reasonably well against the experimental data by Liou et al. [41] and the computational data (WRLES) by Madabhushi and Vanka [39]. It is noted that the experimental data by Liou et al. [41] is extracted at an assumed fully-developed region near the end of the first pass. Streamwise velocity contour and secondary flow motion are observed against the WRLES data (Figure 6.4). According to Madabhushi and Vanka [39], the centerline velocity with a bulk velocity normalization was 1.278 compared to 1.198 from WMLES, which is equivalent to approximately $6 \%$ difference. The vector field indicates multiple 
secondary fluid motions near walls about the diagonal axes. Figure 6.5 shows a comparison plot of the WMLES $u_{r m s}$ data against the experimental data by Liou et al. [41]. Throughout the wall-normal direction, the $u_{r m s}$ profile is somewhat underpredicted with highest differences near walls.

\subsection{Results of Stationary Smooth Duct with U-bend at $\operatorname{Re}=\mathbf{2 5 , 0 0 0}$}

The flow was maintained at a bulk Reynolds number of 25,000 and a bulk rotation number of 0 while varying density ratios from 0 to 0.13 . The stationary simulations with different density ratios are analyzed with a coarse grid size $(620 \times 32 \times$ 32). Figure 6.6 shows variations of Reynolds stresses, $u_{r m s}, v_{r m s}, w_{r m s}$, and $\overline{u^{\prime} v^{\prime}}$, at the first pass inlet with 500 and 1,000 artificial eddies. The $u_{r m s}$ data at the fully-developed region from Liou et al. [41] are used as a comparison, which is seen in Figure 6.6 (a). While WMLES turbulent profiles overlap on top of each other to indicate the consistency of the generated turbulence based on the SEM input, the overall profiles are underpredicted with higher differences near leading and trailing walls against the experimental data. The $v_{r m s}$ and $\overline{u^{\prime} v^{\prime}}$ profiles, as seen in Figure $6.6(\mathrm{~b})$ and (d), indicate less similarity between the fully-developed smooth duct data and the SEM generated profiles compared to the $w_{r m s}$ profile shown in Figure 6.6 (c). Since most of the turbulent energy is contributed to by the streamwise and spanwise turbulent fluctuations, the profiles of turbulent kinetic energy are reasonably well produced by the SEM.

Figure 6.7 shows spanwise velocity profiles of different WMLES simulations and the experimental data by Liou et al. [41] at a bulk Reynolds number of 10,000 compared to 25,000 of WMLES. The significance of the data at $x=12$, which is $5.4 D_{h}$ upstream 
from the bend entrance, is to avoid the entrance effect due to the bend and accurately capture the fully-developed velocity profile. The extracted location of these velocity profiles were carefully chosen based on analyses by Liou et al. [41], and variations of velocity and heat transfer profiles of WMLES simulations near the vicinity of the bend. While WMLES indicate overpredicted velocity values near the centerline, the profiles indicate relatively reasonable values against the experimental data. At the entrance of the bend, the profile indicates velocity bias toward the inner wall due to the flow acceleration. However, the accelerated flow appears to separate near the center of the bend near the inner wall, resulting in significant reduction in velocity as seen in Figure 6.7 (c). The separated flow regains momentum at the exit of the bend where the velocity values are more uniform in the cross-section. As the flow moves downstream in the second pass, the flow approaches a fully-developed state. The velocity profiles compared against the experimental data by Liou et al. [41] indicates closer velocity profiles predictions compared to the velocity profiles at $x=12$ in the first pass for a density ratio of 0 . In addition, for a density ratio of 0.13 , the flow appears to have larger momentum.

Furthermore, streamwise and spanwise turbulent profiles, $u_{r m s}$ and $w_{r m s}$, were examined against the $u_{r m s}$ experimental data as shown in Figure 6.8 (a) and (c), wallnormal and spanwise directions, respectively. While both $u_{r m s}$ profiles near the centerline are relatively well predicted, the $u_{r m s}$ values near walls are underpredicted. In contrast, the $w_{r m s}$ profile is approximately the same among different WMLES results. Notably, these profiles are lower along the spanwise direction due to a wall damping as seen in Figure 6.8 (d). 
Despite the possible differences of the bend geometry between the present study and experiment, the secondary flow motion is examined against the experimental data by Liou and Chen [40]. As seen in Figure 6.9, the secondary flow cells are evenly distributed in wall-normal direction about the centerline with a bias towards the inner wall unlike the experiment data. The direction of secondary flow is from the inner to the outer wall at the center of the duct, resulting in a region of flow impingement on the outer wall. Consequently, heat transfer values are locally enhanced due to high momentum fluid impinging on the outer wall. Overall, the symmetrical secondary flow motions are well predicted compared to the experimental data of Liou and Chen [40].

In addition to the inlet and first pass, streamwise and spanwise turbulence $\left(u_{r m s}\right.$ and $w_{r m s}$ ) within the bend and downstream in the second pass are also analyzed to observe turbulence variations. First, the $u_{r m s}$ profiles are examined. As seen in Figure 6.10 (a), the entrance of the bend indicates a symmetric profile. However, at the center of the bend, the different numbers of SEM eddies show slight differences near the outer wall. As expected, the inner wall indicates slightly higher turbulent fluctuations compared to the outer wall due to mixing, which is discussed in a later section. Overall, there is an increase in $u_{r m s}$ in the bend. At the end of the bend, as shown in Figure 6.10 (c), the $u_{r m s}$ profiles are more uniform across the cross-section, whereas at $x=3.1$ in the second pass, both profiles match well against the experimental data, which indicates that the flow is fully-developed.

The $w_{r m s}$ profiles, as shown in Figure 6.11, also show the spanwise variations. At both the entrance of the bend and downstream of the second pass, turbulent profiles are symmetric about the duct centerline. In contrast, at the center of the bend, the profiles are 
highly non-symmetric with high intensities near the inner wall. Overall, similar to $u_{r m s}$, $w_{r m s}$ is also augmented from its fully-developed value. Coming out the bend, the magnitude is still high but more uniform across the cross-section.

Figure 6.12 shows averaged heat transfer augmentation ratio contour plots on leading, trailing, outer, and inner walls at a density ratio of 0.13 in the vicinity of the bend. Both leading and trailing walls indicate similar heat transfer contours where two distinctive areas of high heat transfer are seen near the center of the bend and the exit of the bend. The outer wall heat also shows high heat transfer in the bend and coming out the bend. High transfer at the entrance to the bend at the inner wall, is followed by a low heat transfer region with near wall localized maximums in the U-bend.

In order to study the effect of different number of inlet eddies and different density ratios, heat transfer results are compared to experiments along the length of the duct. Overall, it is found that the density ratio does not have a large impact on the heat transfer coefficient in the stationary duct. Contrary to expectations, the inlet turbulence (500 and 1,000 eddies) impacts the heat transfer throughout the two passes of the duct, more than the density ratio. Both leading and trailing side heat transfer augmentation ratios have 2 local maxima within the bend, which occur at the center of the bend and at the exit of the bend. This is similar to the trend observed in the experiments. In general, the heat transfer values are overpredicted, especially in the second pass of the duct. The degree of overprediction is higher when 500 eddies are used to generate the inlet turbulence, whereas calculations done with 1,000 eddies seem to agree better with the experimental results. 
The outer wall heat transfer values indicate mixed results. While heat transfer values near the center of the bend compare well with experiments, heat transfer values at the exit of the bend and downstream are overpredicted. The sharp decrease in heat transfer at the outer wall near the entrance to the bend is a consequence of flow separation as the flow encounters diverging section of duct. Figure 6.14, shows the region of flow separation at the outer wall for both density ratios of 0 and 0.13 . The high heat transfer at the outer wall at the middle of the bend is a result of the flow impingement of the secondary flow shown in Figure 6.9. The second larger peak at the exit of the bend is a result of flow impingement on the outer wall as the flow comes out of the bend

At the inner wall, there is an initial increase in heat transfer as the flow enters the bend as a result of flow acceleration, followed by a drop in heat transfer, and then an increase in the second half of the bend after which there is drop as the flow comes out of the bend. Figure 6.15 shows mean flow streamlines in the bend near the inner wall. Going into the bend the mass flow is concentrated near the inner wall with increased mixing as indicated by the concentrated swirling streamlines. This flow cannot follow the contour of the inner bend and separates before reattaching back near the entrance to the second pass. Out of all the predictions, the second pass heat transfer at the inner wall seems to have the largest discrepancy with the experimental results. Experiments decay below the nominal value of unity as the flow develops in the second pass. On the other hand, the predictions show a slight increase initially and decrease slowly into the second pass with values closer to unity. We also note that the experimental results at the inner wall are much lower than the outer wall in the fully-developed region upstream of the bend. In 
fact the augmentation ratio at $\mathrm{x} / \mathrm{Dh}=12$ is 0.7 at the inner wall when it should be close to unity.

\subsection{Results of Rotating Smooth Duct with U-bend at $R e=25,000$ and $R o=0.238$}

The rotational simulations with different density ratios are analyzed with both coarse and fine grid sizes. Similar to stationary analyses in the previous section, flow and heat transfer analyses are done to observe effects of the density ratio differences and grid resolution within the bend. Rotation with constant properties activates Coriolis and centrifugal pumping forces. While Coriolis forces have stabilizing/destabilizing effects at the leading and trailing walls, respectively, centrifugal forces act uniformly across any given cross-section but increase as the radius from the axis of rotation increases. They act in the direction of the driving pressure gradient in the first pass but act against the driving pressure gradient in the second pass. When the density is dependent on temperature, the magnitude of the centrifugal forces now varies in a given cross-section. For outward flow in the first pass, because Coriolis forces decrease the amount of heat transferred on the leading side and increase heat transfer on the trailing side, the fluid temperatures are lower near the trailing side than near the leading side. This leads to a differential in the centrifugal force which is stronger on the trailing side than the leading side because of the ensuing density differences. The higher centrifugal force on the trailing side pumps more fluid near the trailing side, decreasing the momentum of the flow on the leading side. Hence, it works in tandem with the effect of Coriolis forces. In inward flow in the second pass, because the destabilizing effect of Coriolis forces shifts from the trailing to the leading side, centrifugal forces are higher on the leading side than the trailing side. But in 
this case, the centrifugal force acts opposite to the direction of flow and tends to counter the effect of Coriolis forces leading to stabilization on the leading side.

Figure 6.16 shows the variation of streamwise velocity with y-coordinate extending from the trailing to the leading side at different streamwise location in the twopass duct. Results are shown on the coarse and fine grid. It is noted that substantial differences exist between the two grids, even at locations upstream of the grid refinement which is only done in the bend. The reason for this is not known and could be a result of the SEM generated inlet conditions. In spite of this anomalous behavior we can make some general observations. Due to Coriolis forces, all simulations indicate a trailing side biased velocity profile at $\mathrm{x}=12$. The bias increases when the density ratio is 0.13 with the profile on the coarse grid indicating a recirculation region on the leading side of the duct. The general trends are consistent with the expected physics in that the centrifugal buoyancy forces augment the effect of the Coriolis forces for outward flow by adding momentum on the trailing side and extracting momentum from the leading side. In the middle of the bend, Figure 6.16 (c), the profile shape is revered with the streamwise velocity (now W) has more momentum at the leading side. As the flow come out of the bend the profiles are more uniform. The comparison with the experiments of Liou and Chen [40] show the same trends considering the large differences in Reynolds number and geometry. At $x=3$ in the second pass, the profiles are symmetric about the centerline. This trend is not consistent with the physics which would dictate that the profiles maintain their asymmetry due to the effect of Coriolis forces.

For further analyses the spanwise variations of mean velocity profiles is shown in Figure 6.17. Unlike the variation in the y-direction, the spanwise variations are 
symmetric about the center of the duct as the flow approaches the bend. The velocity profiles do not show a large dependence on the grid nor the density ratio. This is not the case in the middle of the bend, where the velocity profile at the outer wall is much higher than at the inner wall clearly showing the effect of centrifugal buoyancy. As the flow exits the bend, mean velocity profiles are still biased toward the outer wall due to the turning flow. The flow downstream indicates blunt and uniform velocity profiles for all simulations.

A secondary fluid motion at the center of the bend is shown in Figure 6.18. The rotational effect is clearly seen in this figure where the secondary motion is biased toward the leading side. Despite the bulk rotation number difference of the present study and Liou et al. [41], the direction and leading side bias of the secondary flow cells agree well. Heat transfer augmentation results are plotted in Figure 6.19 and compared to the experimental data by Wagner et al. [38] at a density ratio of 0.13 . Comparisons of the effect of Coriolis forces only (density ratio 0 ), Coriolis forces and centrifugal buoyancy (density ratio of 0.13 ) and grid dependency at density ratio of 0.13 are considered. As expected, the heat transfer augmentation on the trailing side decreases in the second pass as it increases on the leading side due to the reversal of the effect of Coriolis forces. It is found that the inclusion of centrifugal buoyancy does not affect the heat transfer on the leading side of the duct as much as it affects it on the trailing side. On the trailing side, the inclusion of centrifugal buoyancy increases heat transfer augmentation in both the first and second pass of the duct. At the side walls (outer and inner), centrifugal buoyancy only has the effect of increasing heat transfer augmentation in the second pass. In general, the simulations fail to predict the high experimental heat transfer coefficients in the bend. 
The largest deficiency is on the trailing side in the middle of the bend. The difference between the fine grid and coarse grid is mostly noticeable in the second pass. However, differences also exist in the first pass in spite of the fact that the grid is only refined in the bend. The only other simulation parameter which could affect the simulation results in the first pass is the SEM which generates the inlet turbulence.

\subsection{Conclusions}

The prediction capability of the wall modeled LES used in conjunction with the SEM is evaluated in a developing two-pass smooth duct flow with a U-bend. A stationary duct as well as a rotating duct with and without the inclusion of centrifugal buoyancy is simulated. The simulation results are compared with the heat transfer results of Wagner et al. [38], and flow field measurement by Liou and Chen [40] and Liou et al. [41].

Overall prediction accuracy was reasonable for the stationary duct considering the complexity of performing the simulations with SEM and WMLES. For the rotating duct, larger discrepancies were noted between the predicted and experimental heat transfer coefficients, particularly within the bend and downstream of it. It is not clear whether these discrepancies arise due to the uncertainty in reproducing the correct bend geometry, or inherent shortcomings in the wall model. Additional complications arise from the use of the SEM at the inlet to the computational domain. Ideally, results far downstream of the inlet should not be statistically sensitive to the inlet turbulence, but evidence from the simulations suggest otherwise, especially in the rotating duct. This could be the result of the artificial length scales used to generate the inlet eddies. Further work is needed in this area. 


\subsection{Figures}

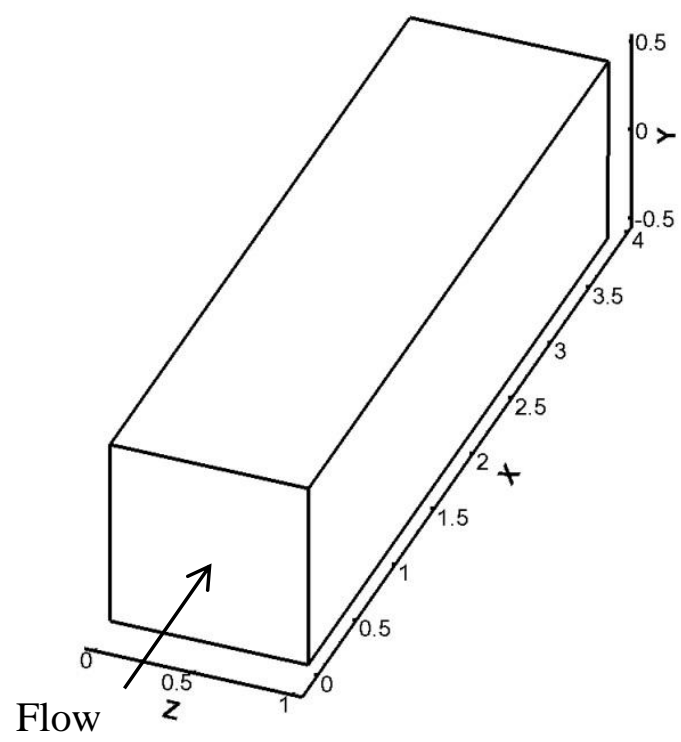

Figure 6.1 The computational domain for the fully-developed smooth duct flow. 


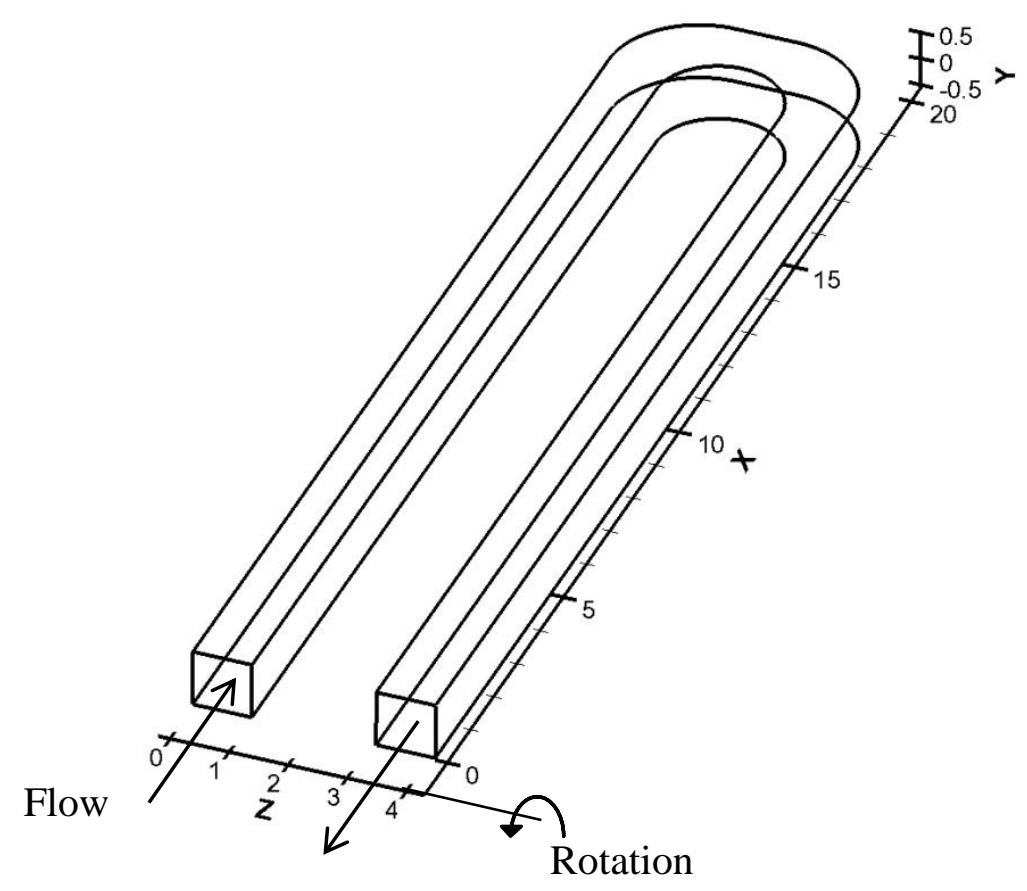

(a)

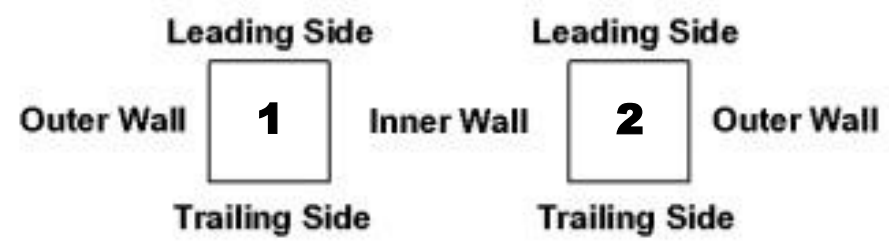

(b)

Figure 6.2 (a) The computational domain for the two-pass smooth duct with a Ubend (b) The cross-sectional view of the computational domain where 1 denotes the first pass and 2 denotes the second pass. 


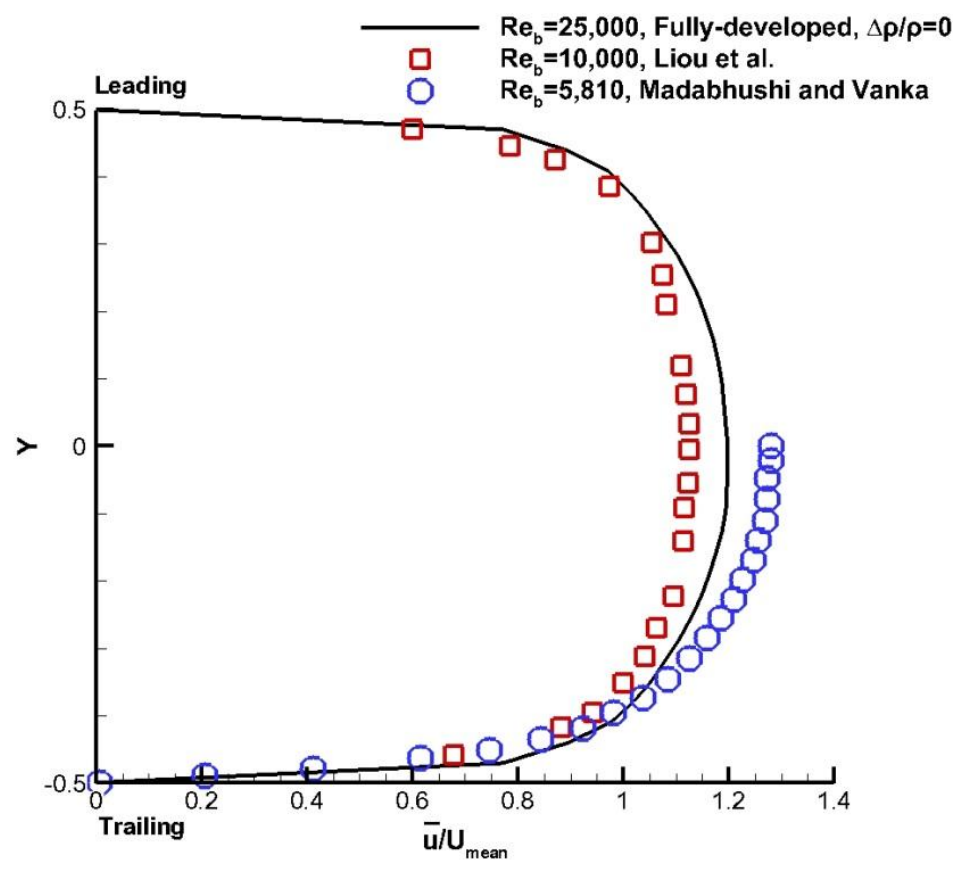

Figure 6.3 The mean velocity profiles of WMLES, experiment, and WRLES, respectively. 


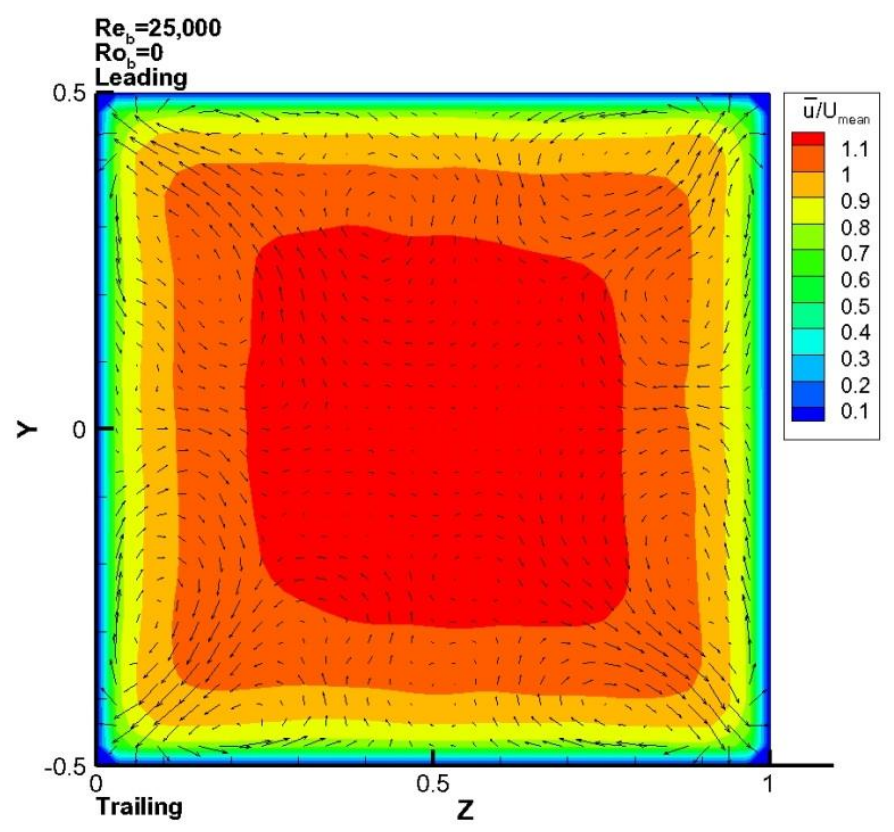

(a)

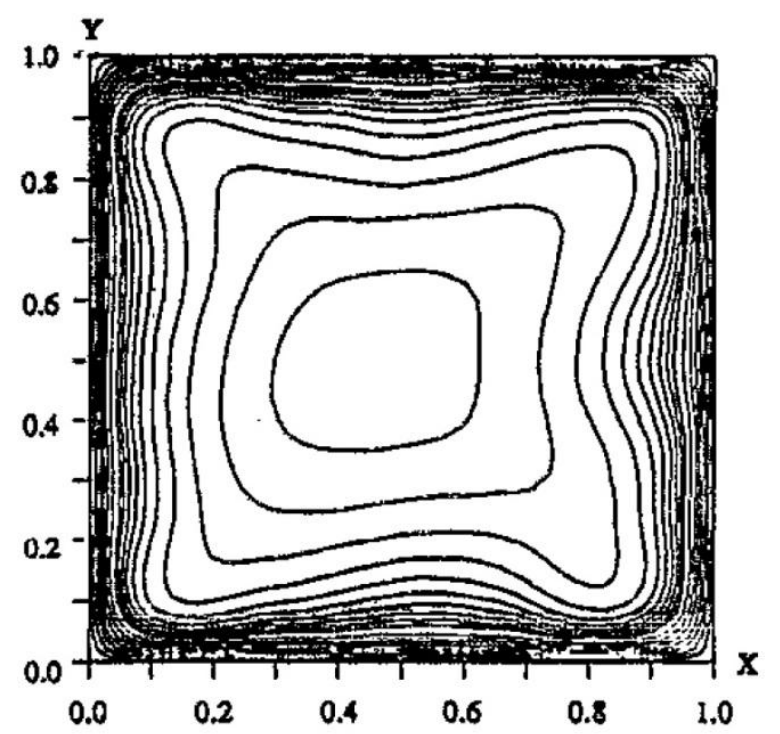

(b)

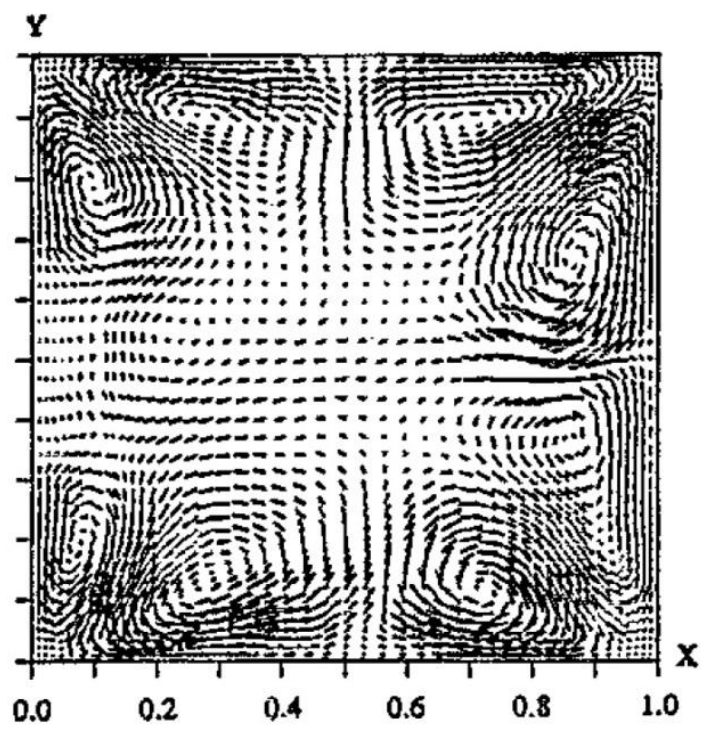

(c)

Figure 6.4 The streamwise velocity contour and secondary flow motion superimposed using contours and vectors: (a) WMLES (b) Contours of streamwise velocity by Madabhushi and Vanka [39] (c) Vector field of secondary flow motion by Madabhushi and Vanka [39]. 


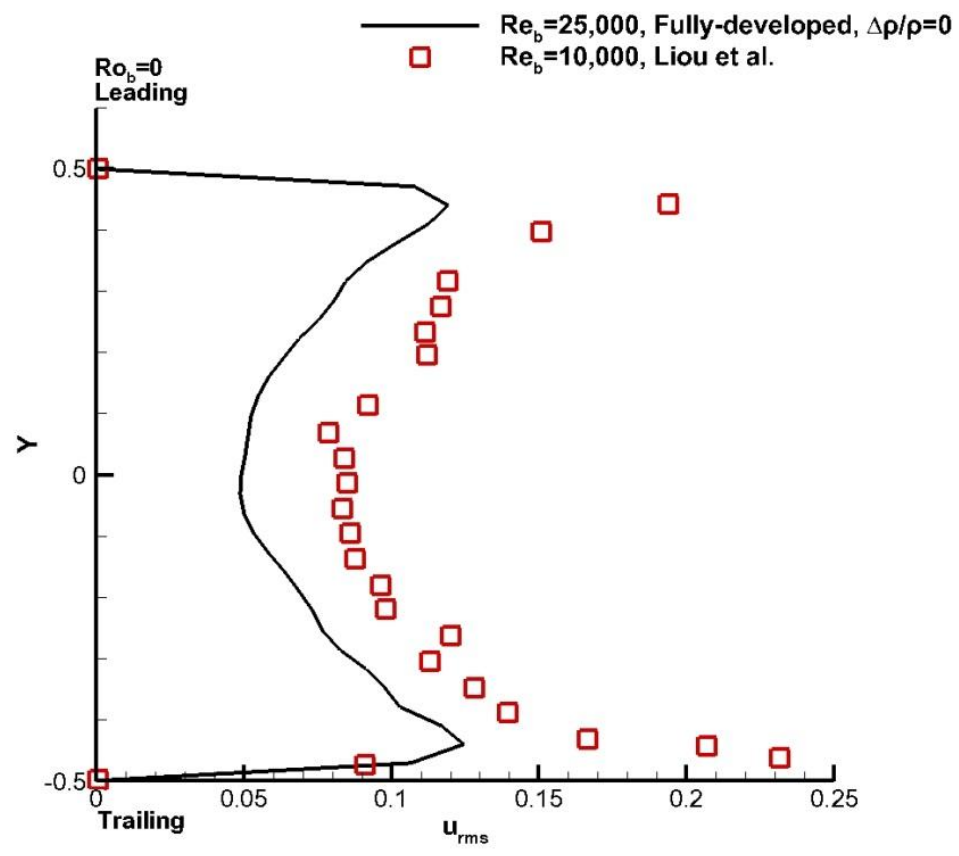

Figure 6.5 The $u_{r m s}$ profile of WMLES and experiment. 


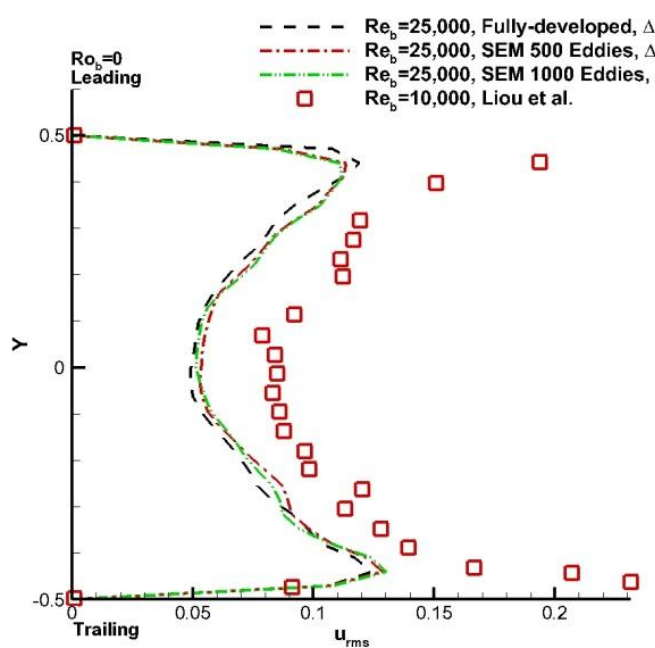

(a)

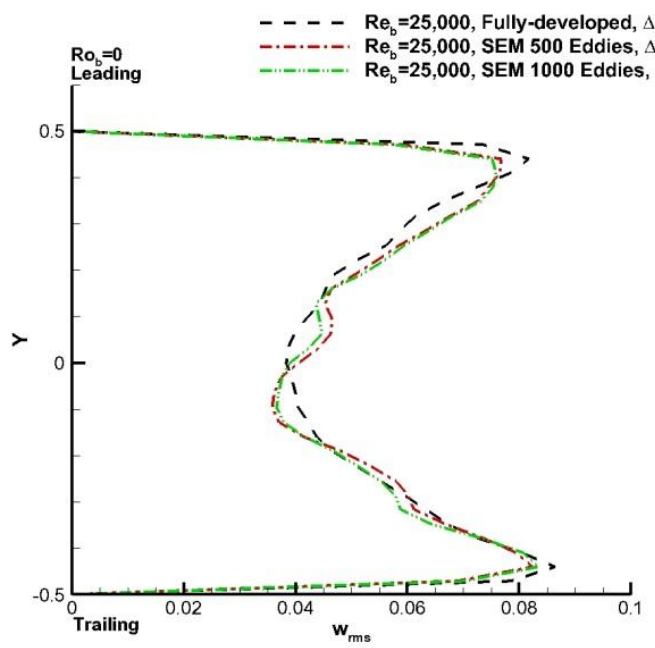

(c)

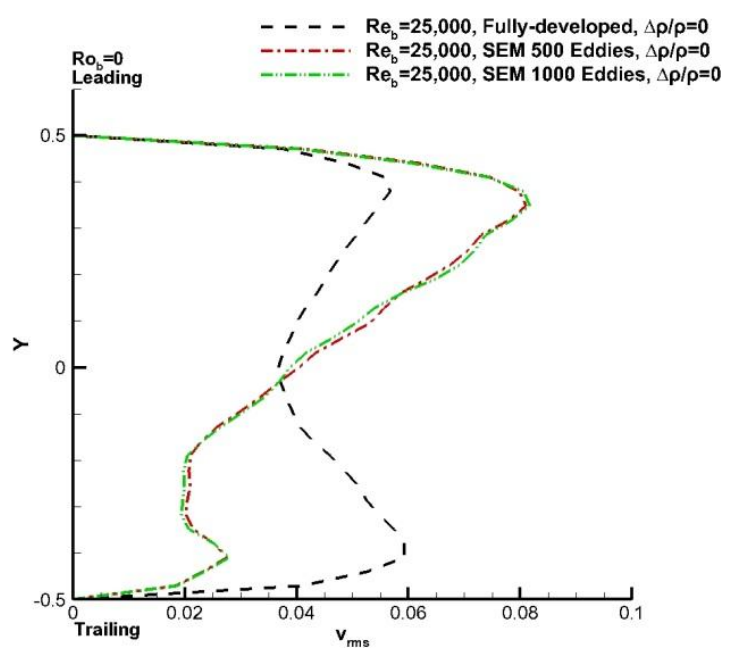

(b)

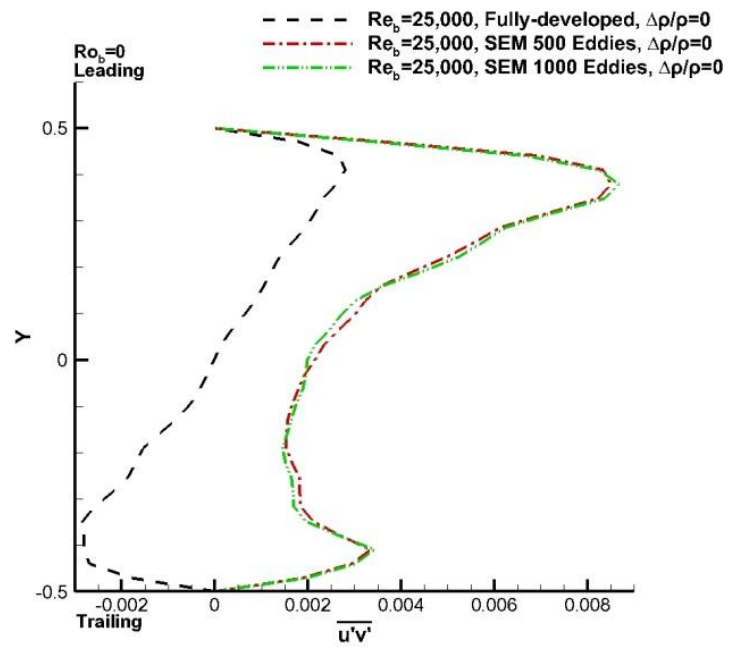

(d)

Figure 6.6 Variations of Reynolds stresses at the first pass inlet for a stationary fully-developed, 500 eddies at a density ratio of 0 , and 1,000 eddies at a density ratio of 0: (a) $u_{r m s}$ (b) $v_{r m s}$ (c) $w_{r m s}$ (d) $u^{\prime} v^{\prime}$. 

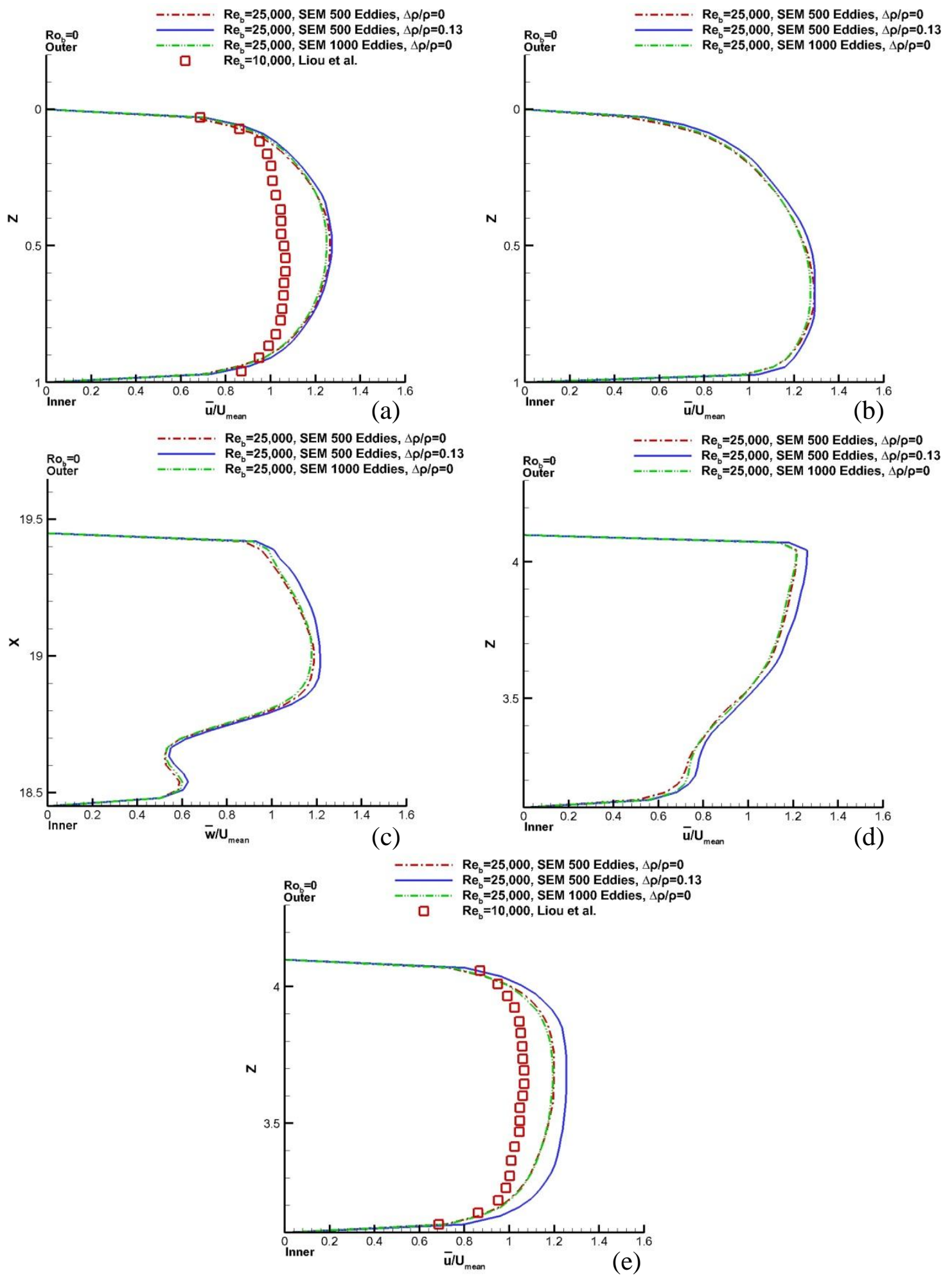

Figure 6.7 Spanwise variations of mean velocity profiles for stationary cases: (a) $x=$ 12 first pass (b) entrance of the bend (c) center of the bend (d) end of the bend (e) $x$ $=3.1$ second pass. 


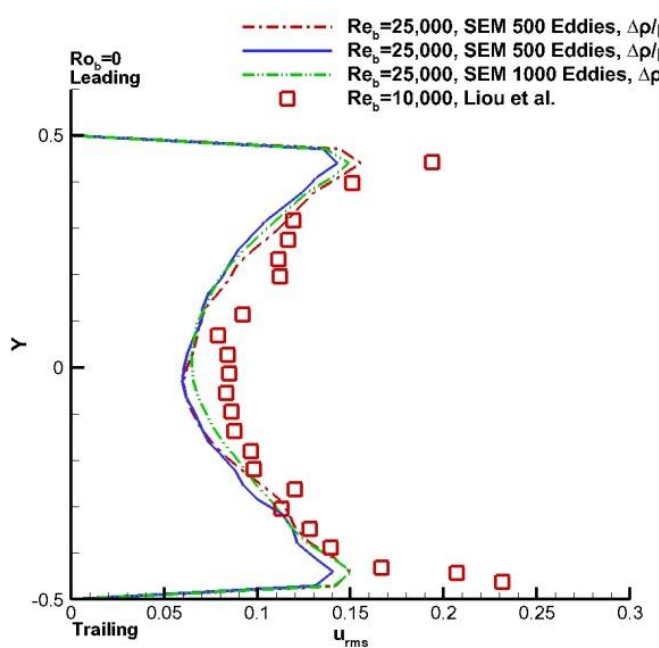

(a)

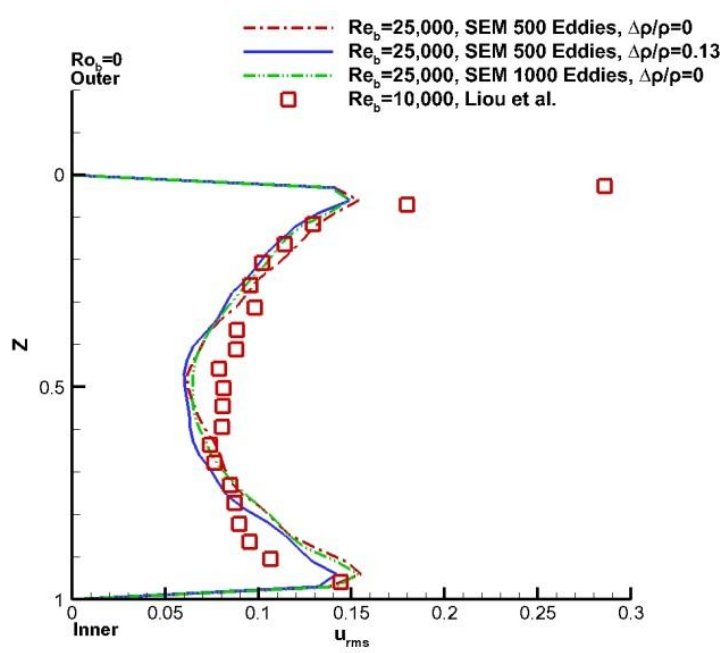

(c)

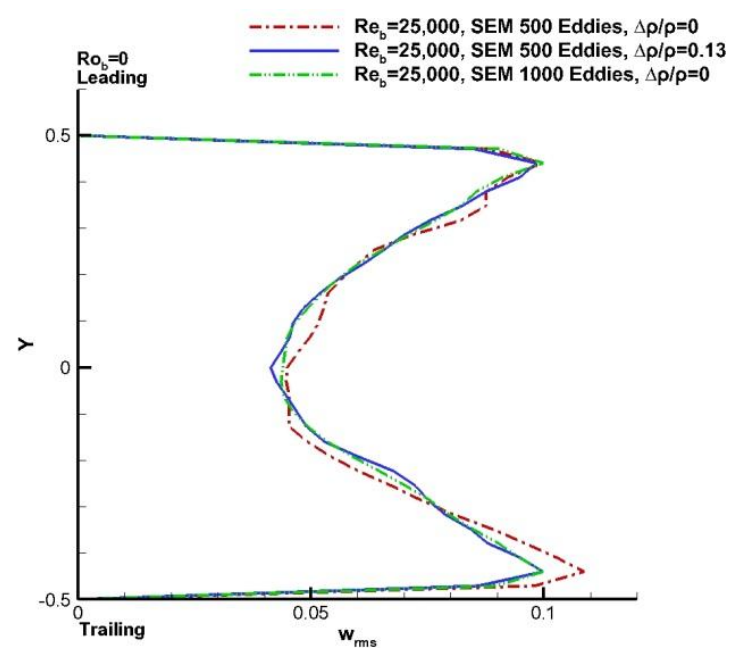

(b)

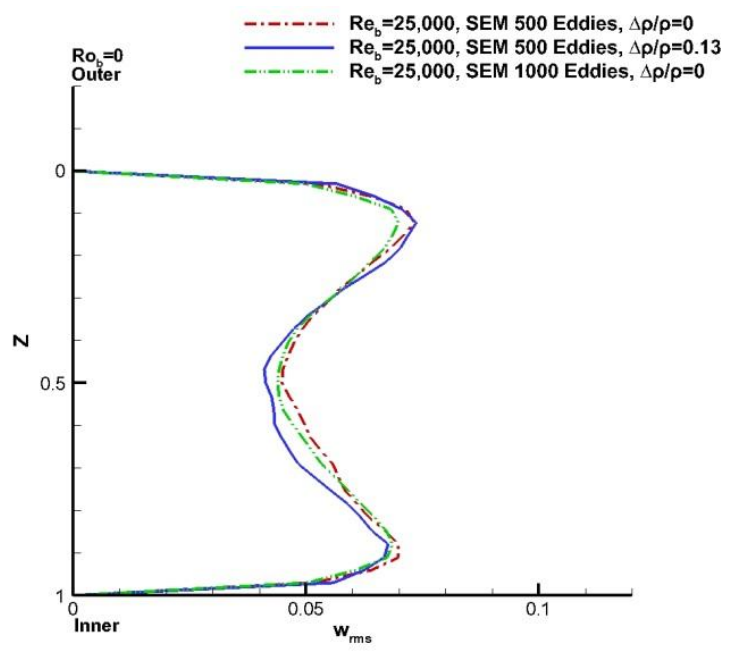

(d)

Figure 6.8 The variation of turbulent profiles for stationary cases at $x=12$ (first pass). 500 eddies, 1,000 eddies, and experimental data are shown: (a) $u_{r m s}$ along $y-$ direction (b) $w_{r m s}$ along y-direction (c) $u_{r m s}$ along z-direction (d) $w_{r m s}$ along zdirection. 


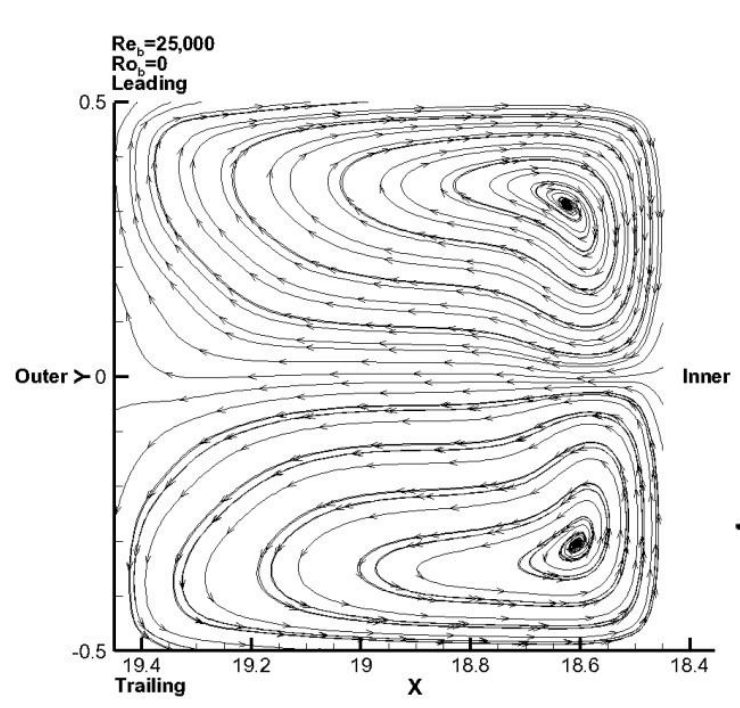

(a)

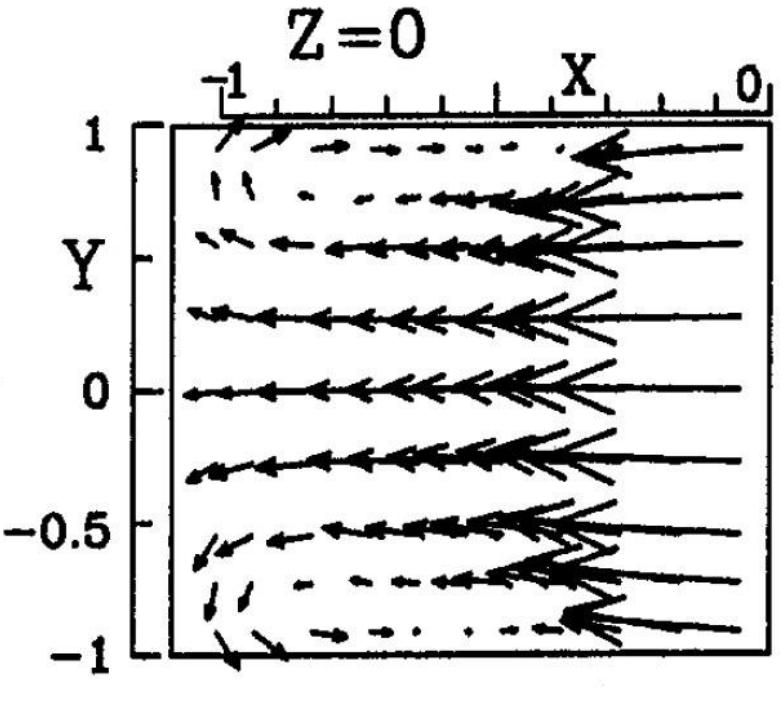

(b)

Figure 6.9 The secondary flow motion of a stationary case with 500 eddies and a density ratio of 0 in the middle of the bend $(z=2.05)$ : (a) WMLES (b) Liou and Chen [40]. 


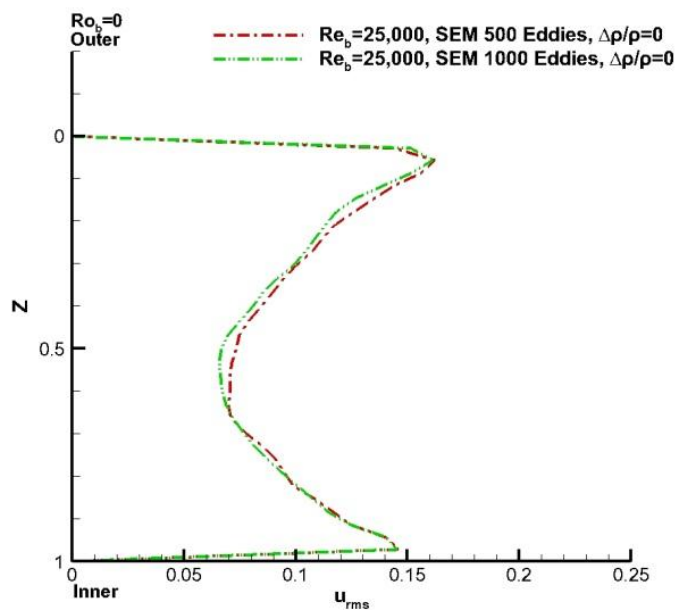

(a)

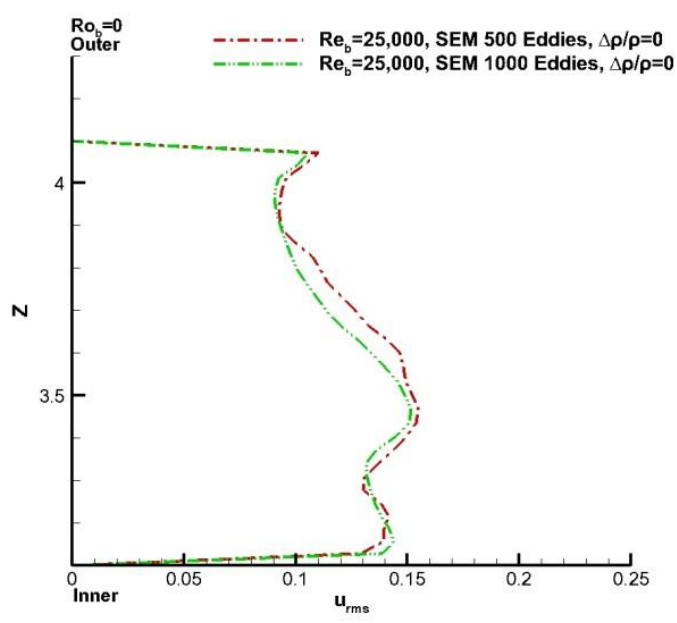

(c)

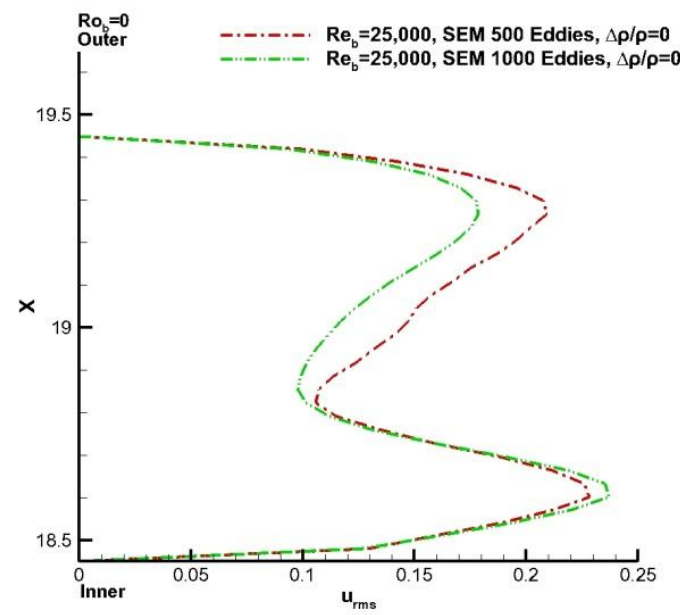

(b)

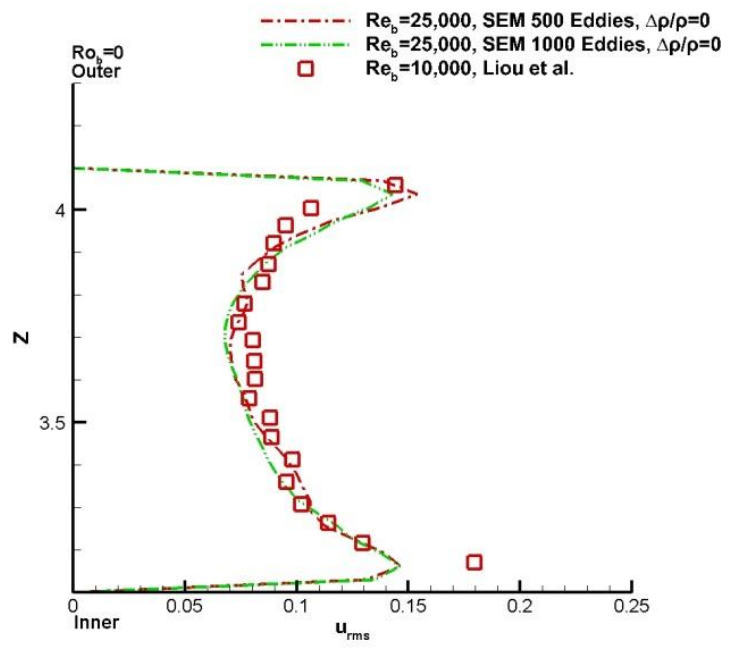

(d)

Figure 6.10 The variation of $\boldsymbol{u}_{r m s}$ in two-passes for a stationary periodic, 500 eddies at a density ratio of 0 and 1,000 eddies at a density ratio of 0: (a) Entrance of the bend (b) Center of the bend (c) Exit of the bend (d) $x=3.1$ second pass. 


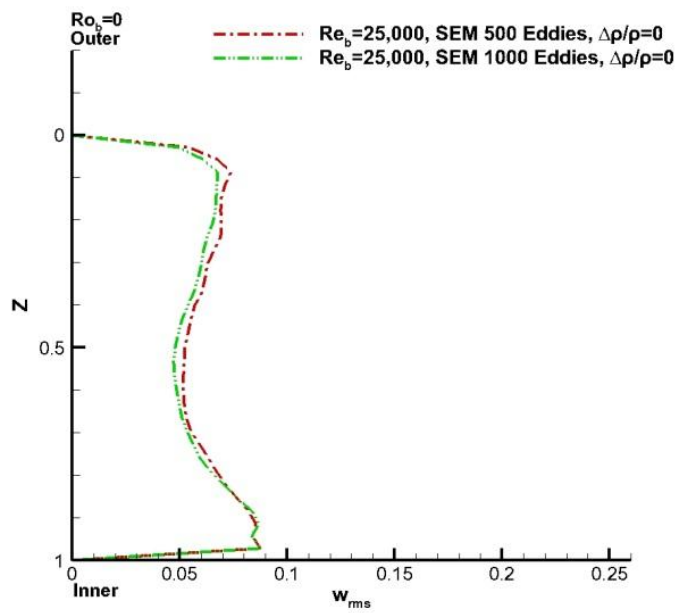

(a)

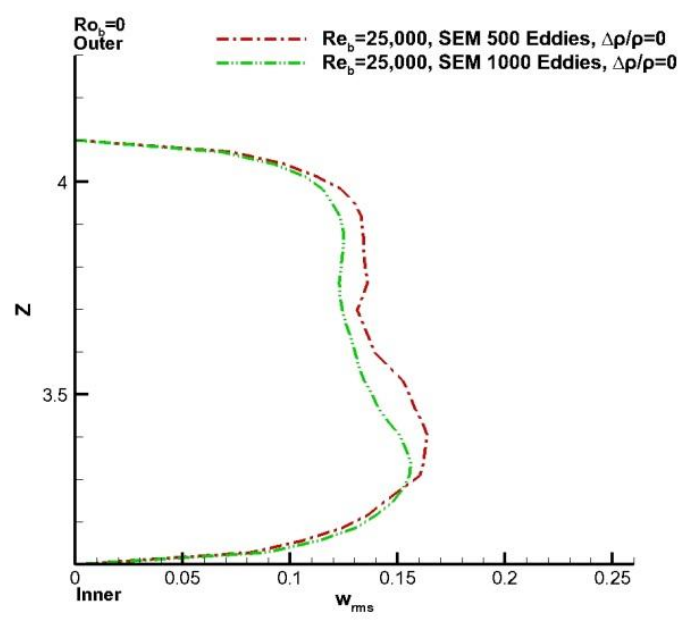

(c)

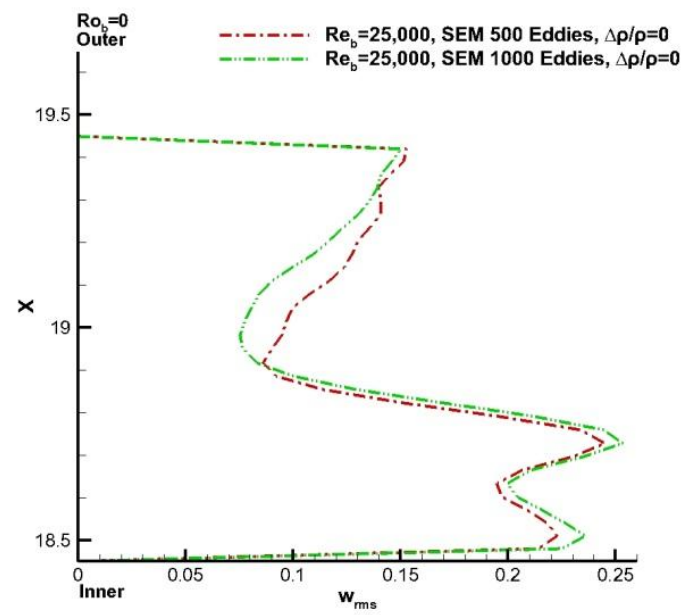

(b)

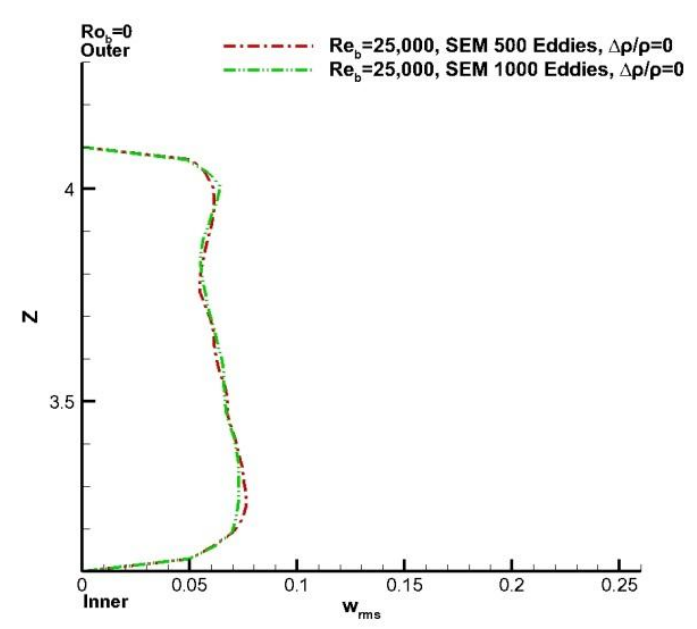

(d)

Figure 6.11 The variation of $w_{r m s}$ in two-passes for a stationary periodic, 500 eddies at a density ratio of 0 and 1,000 eddies at a density ratio of 0: (a) Entrance of the bend (b) Center of the bend (c) Exit of the bend (d) $x=3.1$ second pass. 


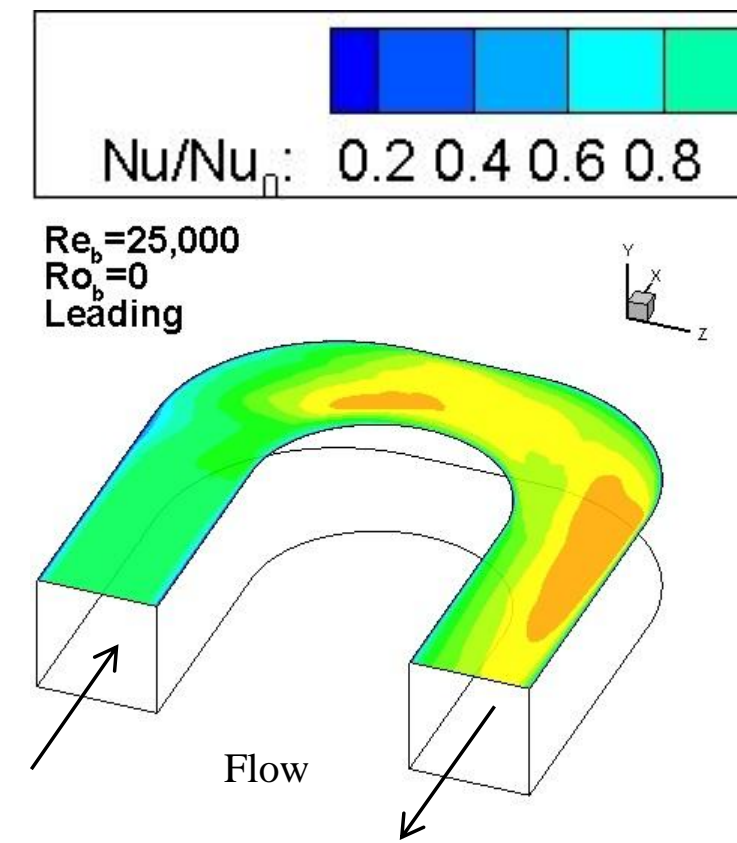

(a)

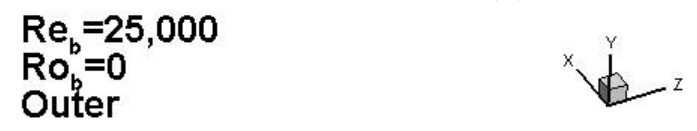

Flow

(c)
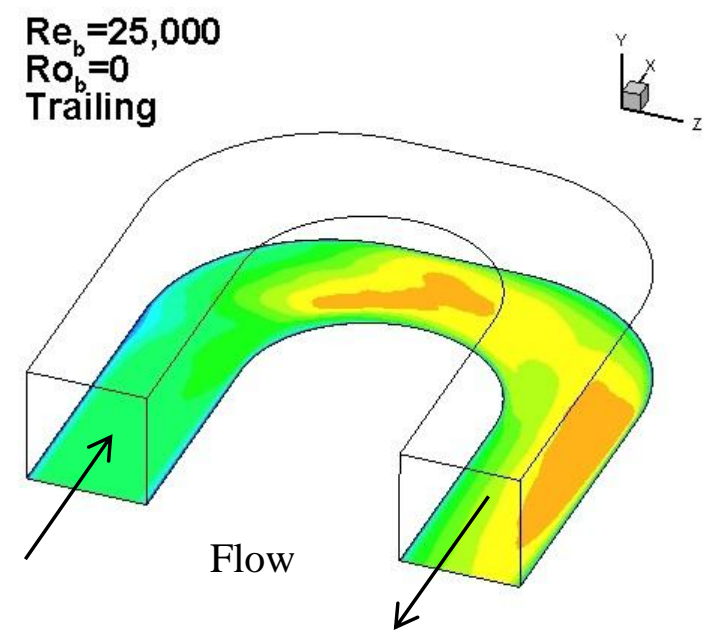

(b)

$$
\begin{aligned}
& \mathrm{Re}_{\mathrm{b}}=25,000 \\
& \mathrm{Ro}_{\mathrm{b}}=0 \\
& \text { Inner }
\end{aligned}
$$

$\mathrm{H}^{\mathrm{C}}$

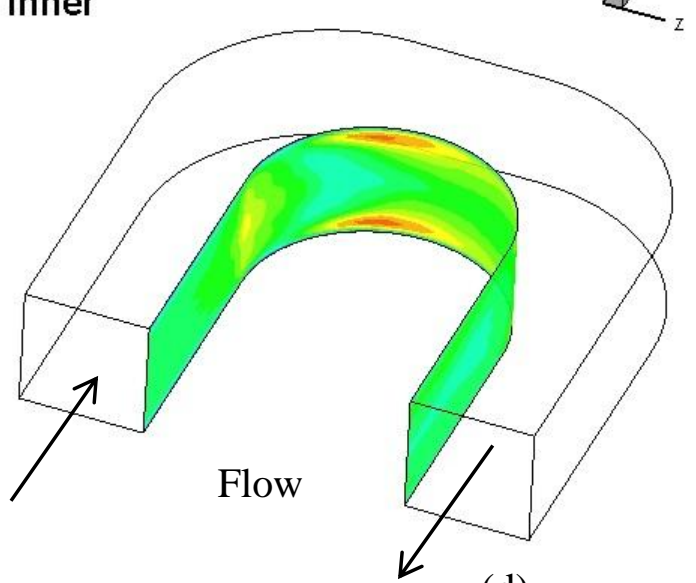

(d)

Figure 6.12 Averaged heat transfer augmentation ratio contour plots on all 4 walls at a density ratio of 0.13 for a stationary simulation: (a) Leading wall (b) Trailing wall (c) Outer wall (d) Inner wall. 


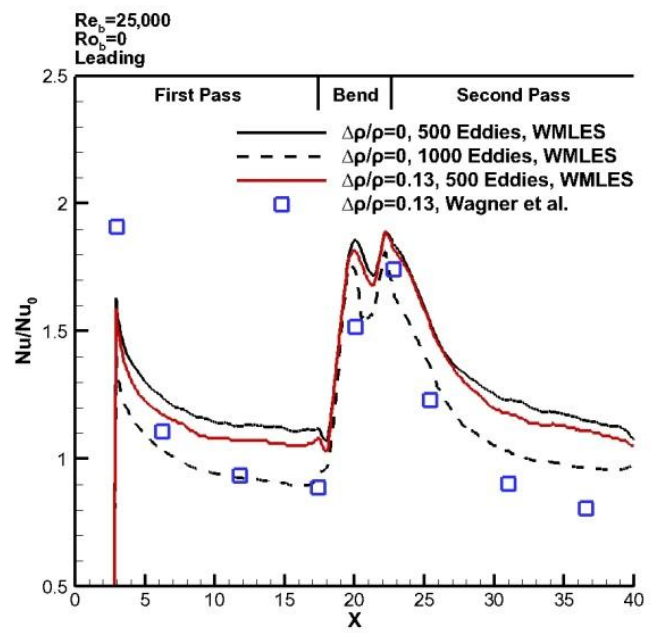

(a)

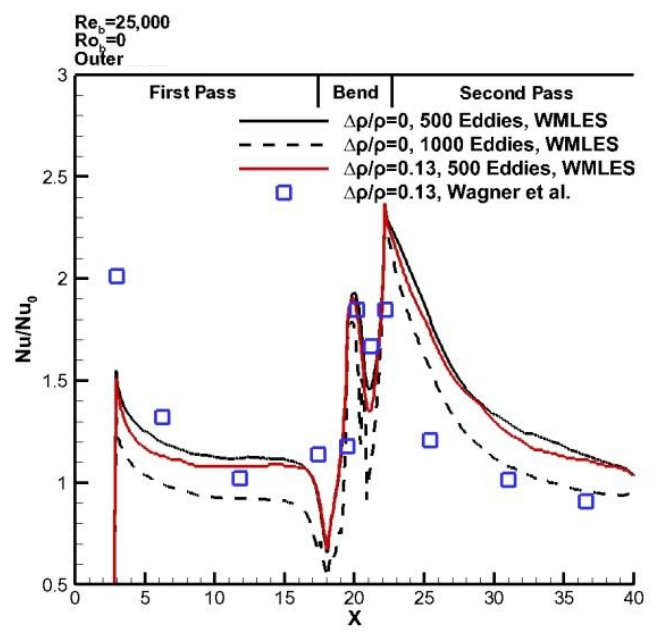

(c)

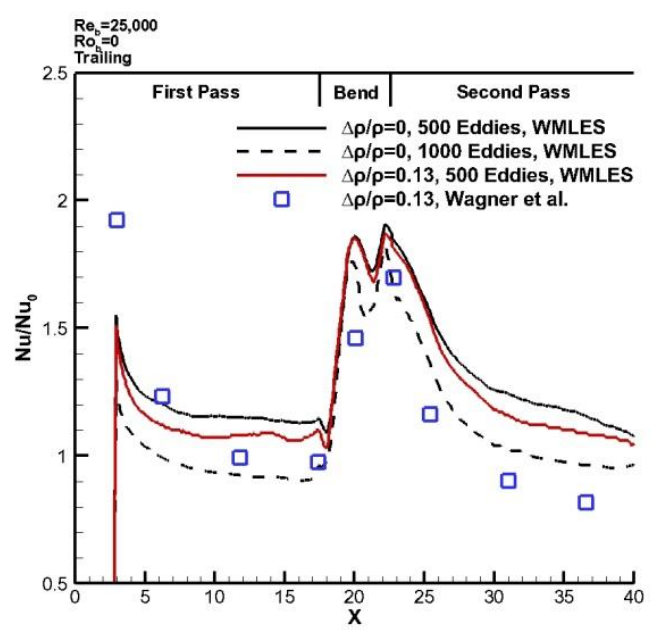

(b)

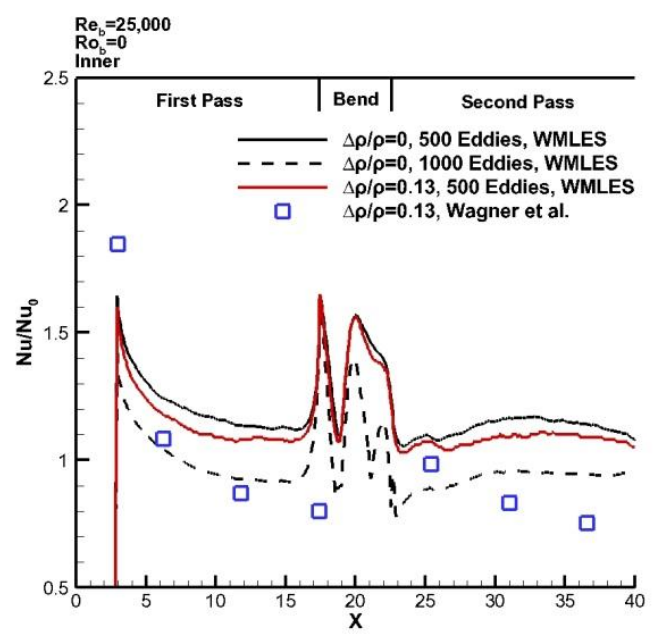

(d)

Figure 6.13 Heat transfer augmentation ratio variations of two-pass smooth duct with U-bend for a stationary case: (a) leading side (b) trailing side (c) outer wall (d) inner wall. 


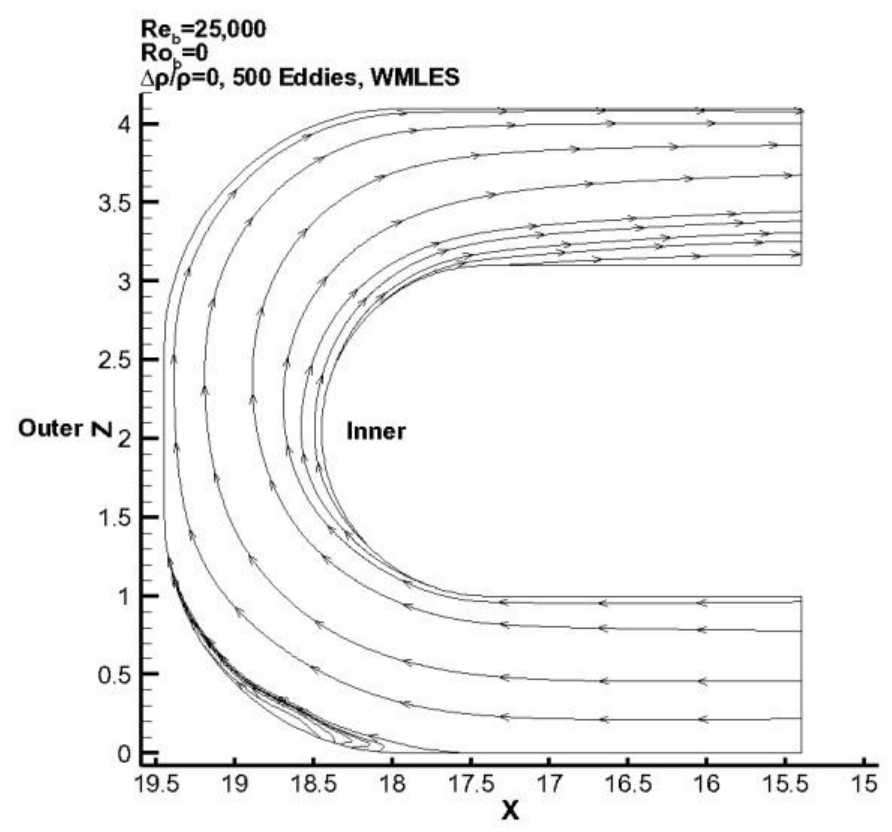

(a)

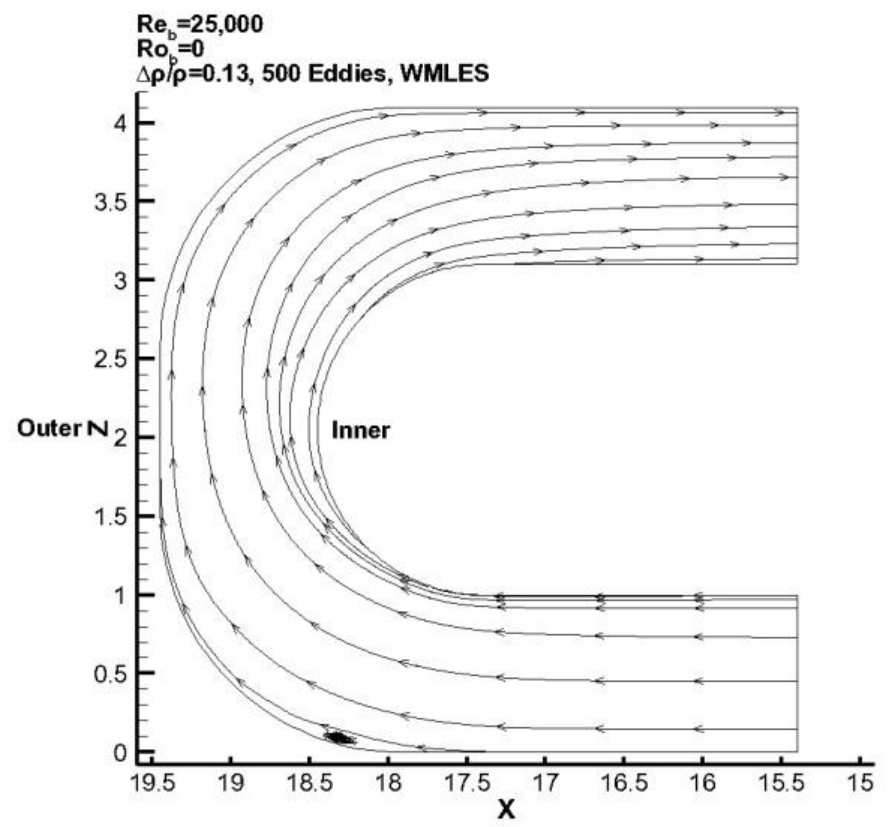

(b)

Figure 6.14 The streamlines at $z=0.25$ : (a) a density ratio of 0 (b) a density ratio of

\subsection{3.}


$\operatorname{Re}_{\mathrm{b}}=\mathbf{2 5 , 0 0 0}$

$\mathrm{Ro}_{\mathrm{O}}=0$

$\Delta \rho / \rho=0,500$ Eddies, WMLES

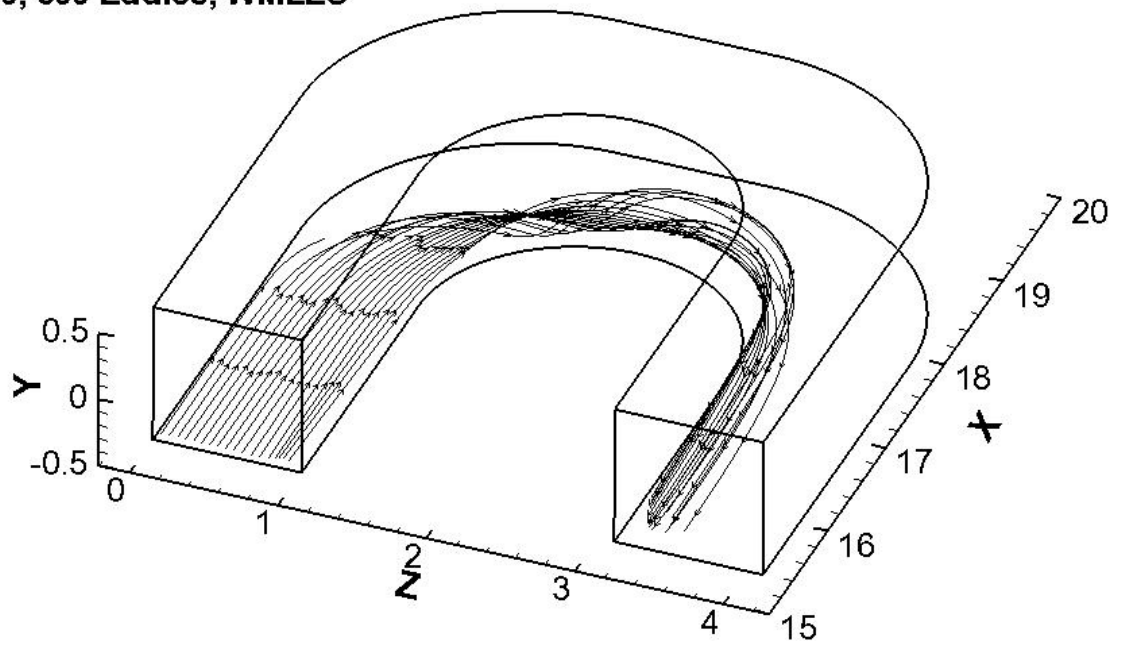

Figure 6.15 The streamlines at different locations for a density ratio of 0 at $y=0.06$. 

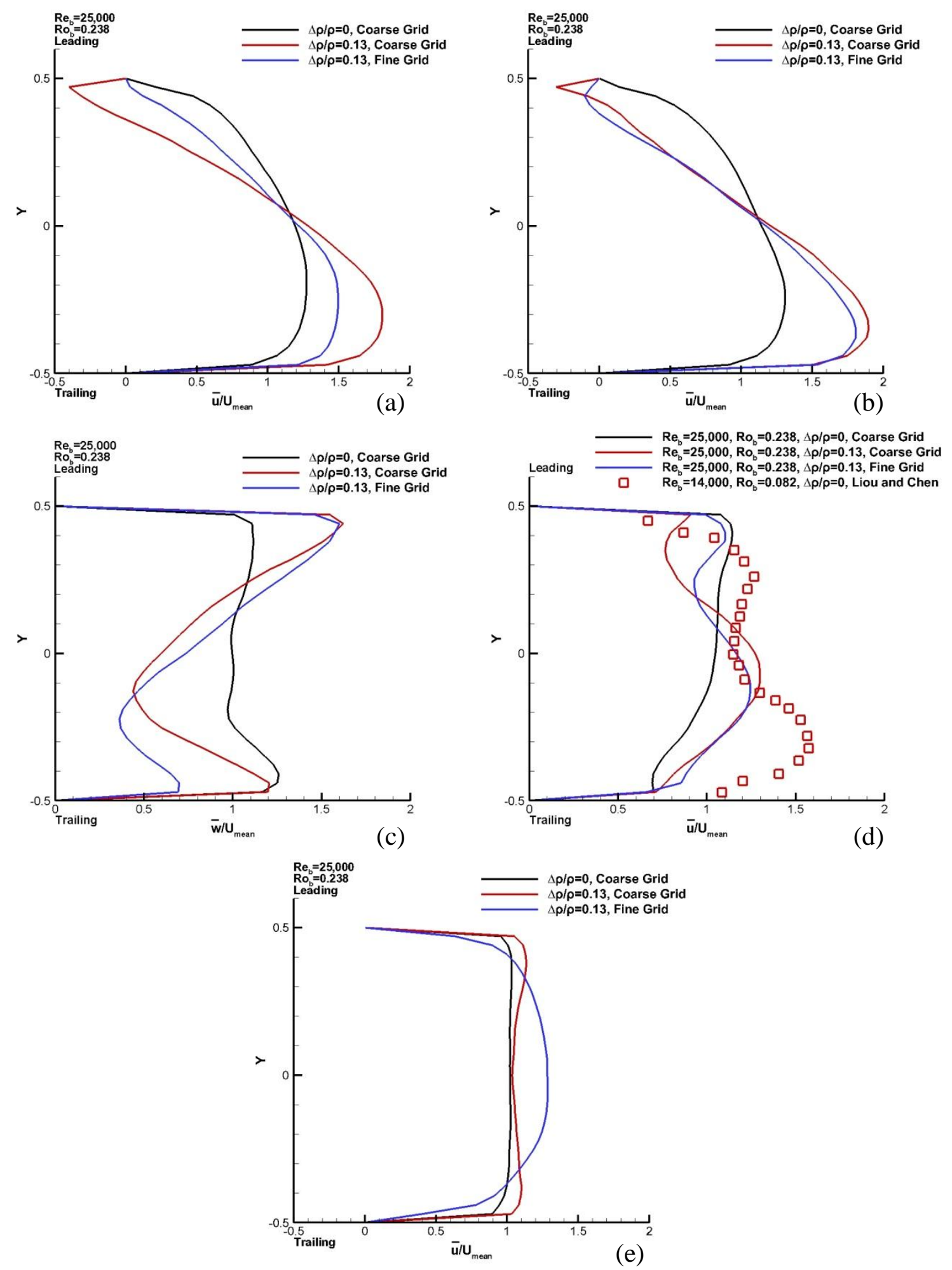

Figure 6.16 Wall-normal variations of streamwise velocity profiles for rotational cases at different locations for 500 eddies: (a) $x=12$ first pass (b) entrance of the bend (c) center of the bend (d) end of the bend (e) $x=3.1$ second pass. 

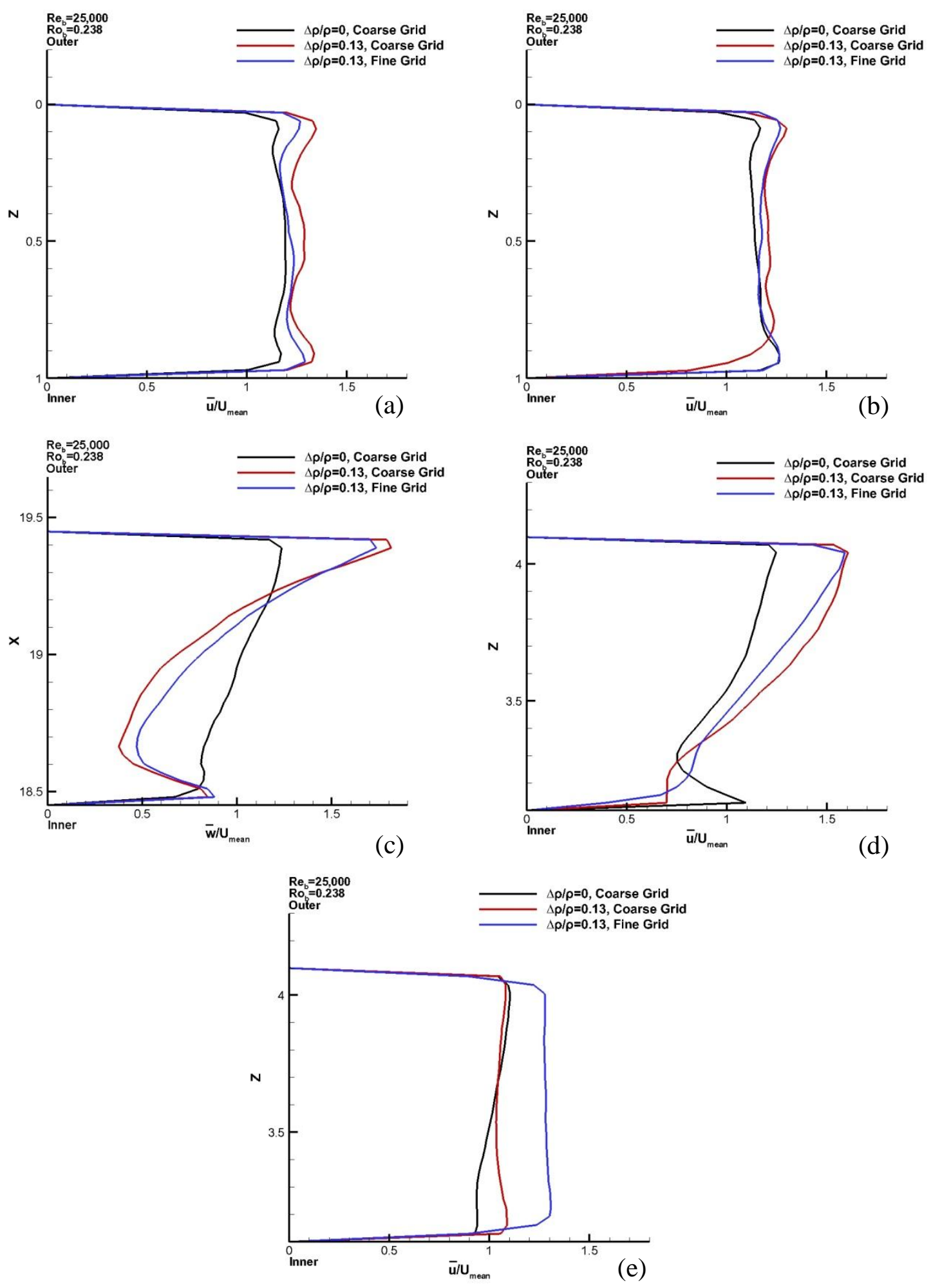

Figure 6.17 Spanwise variations of streamwise velocity profiles for rotational cases at different locations for 500 eddies: (a) $x=12$ first pass (b) entrance of the bend (c) center of the bend (d) end of the bend (e) $x=3.1$ second pass. 


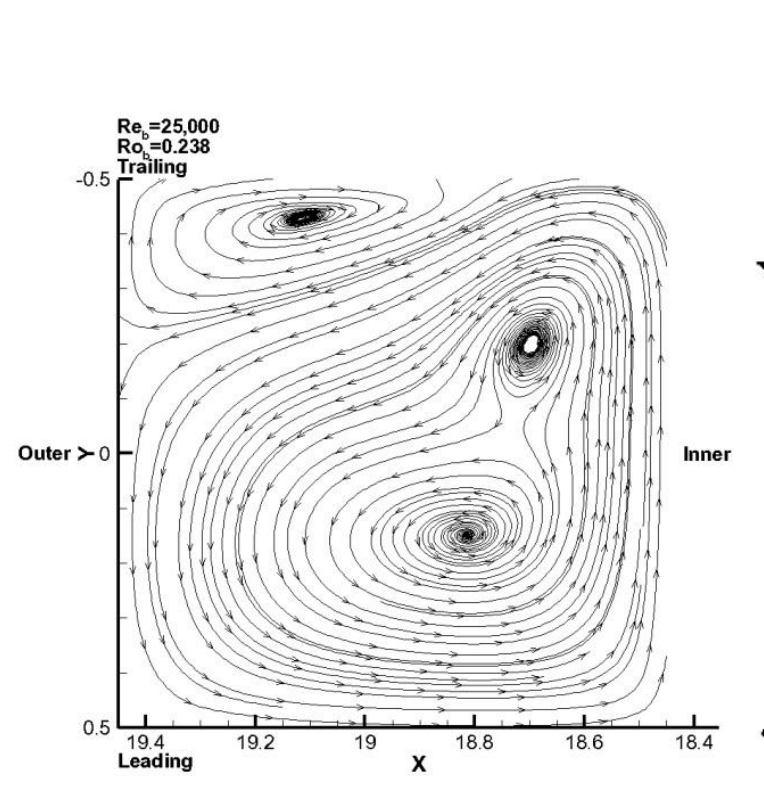

(a)

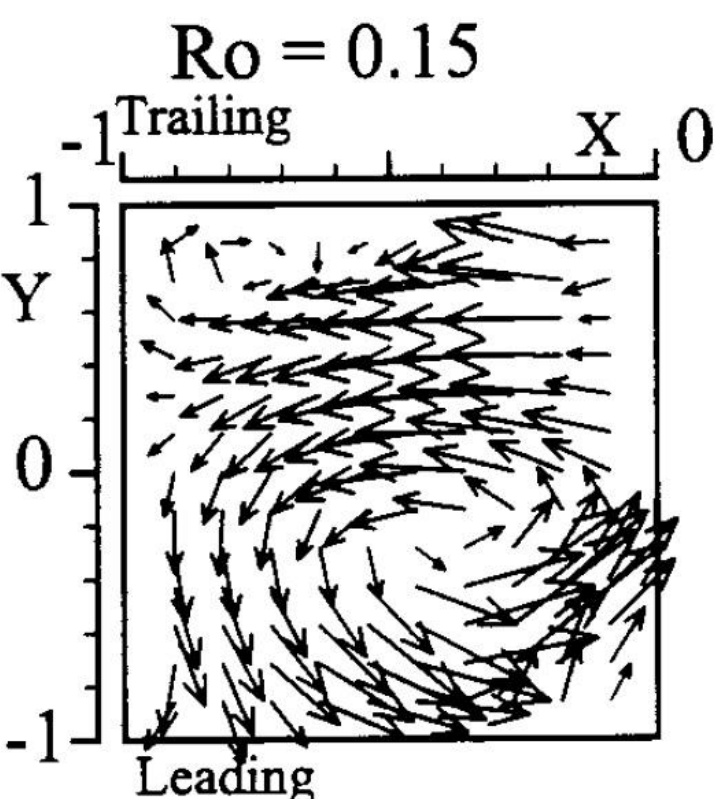

(b)

Figure 6.18 The secondary flow motion of 500 eddies with a density ratio of 0 in the middle of the bend $(z=2.05)$ : (a) a bulk rotation number of 0.238 with WMLES (b) a bulk rotation number of 0.15 from Liou et al. [41]. 


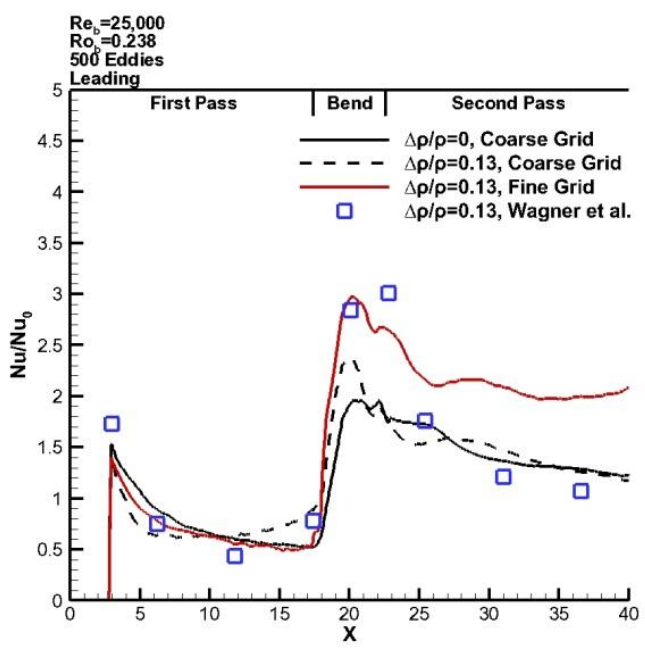

(a)

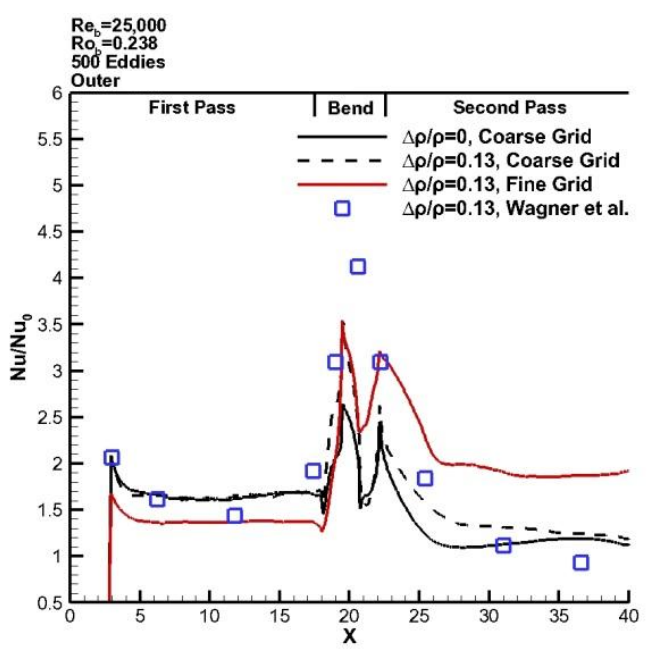

(c)

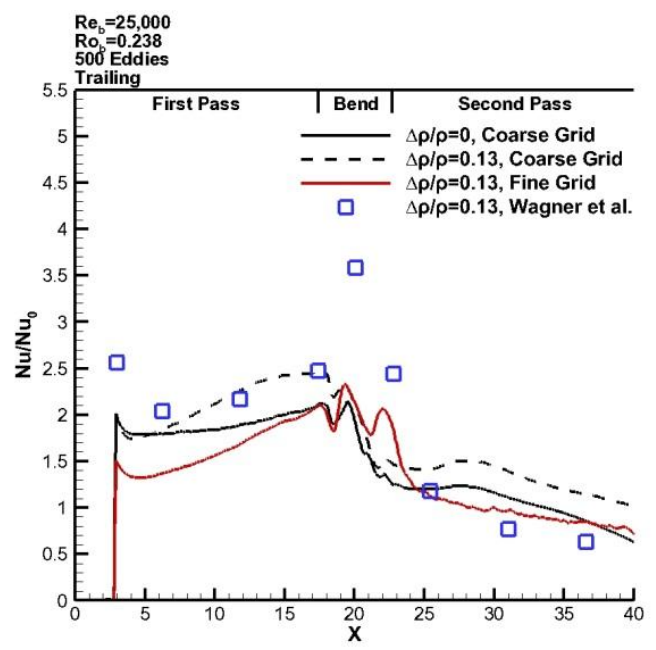

(b)

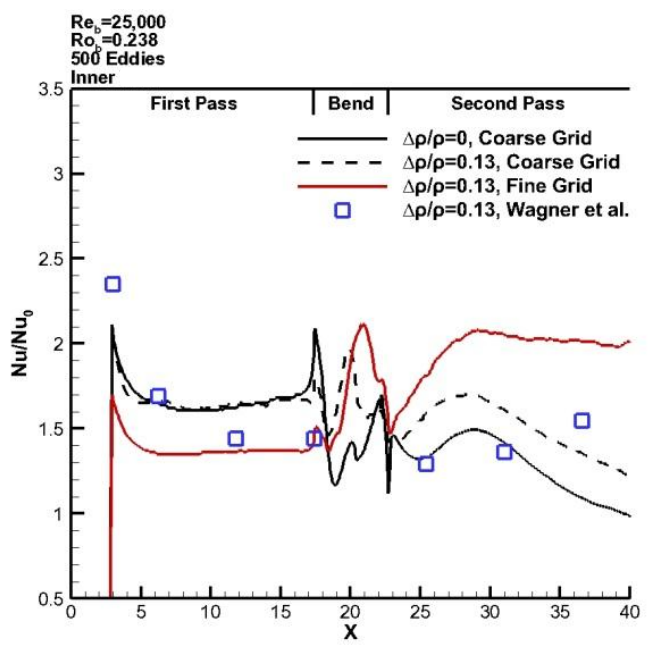

(d)

Figure 6.19 Heat transfer augmentation ratio variations of two-pass smooth duct with U-bend for a bulk rotation number of 0.238 : (a) leading side (b) trailing side (c) outer wall (d) inner wall. 


\section{Chapter 7 Summary and Conclusions}

Wall modeled LES was applied to different geometries presented in this thesis. Due to the large grid requirements of wall resolved LES near walls, the computational effort to simulate highly turbulent flows is significant. In order to alleviate this problem, a simplified boundary layer type of equation is solved near walls with turbulence modeling to minimize the computational effort in LES.

Turbulent channel flow and a $90^{\circ}$ non-staggered ribbed duct with rotation indicated favorable results against both experimental and computational data from the literature. Specifically, friction velocity and heat transfer augmentation ratios over different rotation numbers were within $10-15 \%$. Mean and turbulent flow behavior was found to be well predicted against wall resolved LES results.

Unlike turbulent channel flow and $90^{\circ}$ non-staggered ribbed duct results, a $45^{\circ}$ staggered ribbed duct simulation indicated deviations in mean velocity profiles across the computational domain due to the non-symmetric secondary fluid motion. However, heat transfer results showed reasonable agreement against the experimental data with a clear indication of the helical vortex in the spanwise direction.

The two-pass smooth duct with a U-bend heat transfer results were quite well predicted for the stationary case. However, discrepancies were found between the predicted and experimental heat transfer augmentation ratios for rotating simulations within the bend. These discrepancies could be due to the uncertainty in reproducing the correct bend geometry, inherent shortcomings in the wall model, or the artificial turbulent length scales used to generate inlet turbulence. Future work should be undertaken in 
isolating the effect of SEM on downstream flow prediction, enhancing the wall model by solving for the normal velocity component in the boundary layer assumption. 


\section{Bibliography}

[1] Patil, S. and Tafti, D.K., Wall modeled large eddy simulations of complex high Reynolds number flows with synthetic inlet turbulence. International Journal of Heat and Fluid Flow, 2011.

[2] Patil, S., Large Eddy Simulations of high Reynolds number Complex Flows with Synthetic Inlet Turbulence. 2010.

[3] Tafti, D.K., Time-accurate techniques for turbulent heat transfer analysis in complex geometries, Advances in Computational Fluid Dynamics and Heat Transfer. Developments in Heat Transfer, ed. R. Amano and B. Sunden. 2010, Southampton, UK: WIT PRESS.

[4] White, F.M., Viscous Fluid Flow. Second ed. 1991: McGraw-Hill.

[5] Smagorinsky, J., General Circulation Experiments with the Primitive Equations. I. The Basic Experiment. Monthly Weather Review 91, 1963: p. 99-164.

[6] Germano, M., Piomelli, U., Moin, P., and Cabot, W.H., A dynamic subgrid-scale eddy viscosity model. Phys. Fluids, 1991. 3: p. 1760-1765.

[7] Lilly, D.K., A Proposed Modification of the Germano Subgrid-Scale Eddy Viscosity Model. Phys. Fluids A, 1992(4-3): p. 633-635.

[8] Najjar, F.M. and Tafti, D.K., Study of Discrete Test Filters and Finite Difference Approximations for the Dynamic Subgrid-Scale Stress Model. Phys Fluids, 1996. 8: p. 1076-1088.

[9] Moin, P., Squires, K., Cabot, W., and Lee, S., A Dynamic Sub-Grid-Scale Model for Compressible Turbulence and Scalar Transport. Phys. Fluids A, 1991(3-11): p. 2746-2757.

[10] Thompson, J.F., Warsi, Z.U.A., and Mastin, C.W., Numerical Grid Generation Foundations and Applications. 1985: Elsevier Science Publishing Co., Inc.

[11] Kays, W.M., Turbulent Prandtl number. Where are we? Journal of Heat Transfer, 1994. 116(2): p. 284-295.

[12] Jarrin, N., Benhamadouche, S., Laurence, D., and Prosser, R., A synthetic-eddy method for generating inflow conditions for large-eddy simulations. International Journal of Heat and Fluid Flow, 2007(27): p. 585-593.

[13] Johnston, J.P., Halleen, R.M., and Lezius, D.K., Effects of spanwise rotation on the structure of two-dimensional fully developed turbulent channel flow. J. Fluid Mech., 1972. 56(3): p. 533-557.

[14] Kristoffersen, R. and Andersson, H.I., Direct simulations of low-Reynolds-number turbulent flow in a rotating channel. J. Fluid Mech., 1993. 256: p. 163-197.

[15] Tafti, D.K. and Vanka, S.P., A numerical study of the effects of spanwise rotation on turbulent channel flow. Phys. Fluids A, 1991(3 (4)).

[16] Miyake, Y. and Kajishima, T., Numerical simulation of the effects of Coriolis force on the structure of turbulence. Bull. JSME, 1986b. 29(3341-3346, 33473351).

[17] Piomelli, U. and Scalo, C., Subgrid-scale modelling in relaminarizing flows. Fluid Dynamics Research, 2010(42): p. 1-14.

[18] Kim, J., Moser, R., and Mansour, N., Direct Numerical Simulation of turbulent channel flow up to Re $\tau=590$. Physics of Fluids, 1989(11(4)): p. 943-945. 
[19] Piomelli, U., High Reynolds number calculations using the dynamic subgrid-scale stress model. Physics of Fluids, 1993. 5(6): p. 1484-1490.

[20] Lida, O., Fukudome, K., Iwata, T., and Nagano, Y., Low Reynolds number effects on rotating turbulent Poiseuiile floow. Physics of Fluids, 2010(22).

[21] Grundestam, O., Wallin, S., and Johansson, A.V., Direct numerical simulations of rotating turbulent channel flow. J. Fluid Mech., 2008. 598: p. 177-199.

[22] Alvelius, K., Studies of turbulence and its modelling through large eddy-and direct numerical simulation, in Department of mechanics. 1999, KTH Royal Institute of Technology.

[23] Tafti, D.K., Evaluating the role of subgrid stress modeling in a ribbed duct for the internal cooling of turbine blades. International Journal of Heat and Fluid Flow, 2004. 26(1): p. 92-104.

[24] Rau, G., Cakan, M., Moeller, D., and Arts, T., The Effect of periodic ribs on the local aerodynamic and heat transfer performance of a straight cooling channel. ASME Journal of Turbomachinery, 1998(120): p. 368-375.

[25] Abdel-Wahab, S., Large Eddy Simulation of Flow and Heat Transfer in a Staggered $45^{\circ}$ Ribbed Duct and a Rotating 90 Ribbed Duct. 2003.

[26] Sewall, E.A., Tafti, D.K., Graham, A.B., and Thole, K.A., Experimental validation of large eddy simulations of flow and heat transfer in a stationary ribbed duct. International Journal of Heat and Fluid Flow, 2006. 27(2): p. 243-258.

[27] Fann, S., Yang, W.-J., and Zhang, N., Local heat transfer in a rotating serpentine passage with rib-roughened surfaces. Int. J. Heat Mass Transfer, 1994. 37(2): p. 217-228.

[28] Ekkad, S.V. and Han, J.-C., Detailed heat transfer distributions in two-pass square channels with rib turbulators. Int. J. Heat Mass Transfer, 1997. 40(11): p. 2525-2537.

[29] Viswanathan, A.K. and Tafti, D.K., Investigation of Detached Eddy Simulations in Capturing the Effects of Coriolis Forces and Centrifugal Buoyancy in Ribbed Ducts. J. Heat Transfer, 2007. 129(7): p. 778-789.

[30] Wagner, J.H., Johnson, B.V., Graziani, R.A., and Yeh, F.C., Heat Transfer in Rotating Serpentine Passages with Trips Normal to the Flow. J. Turbomachinery, 1992(114): p. 847-857.

[31] Parsons, J.A., Han, J.-C., and Zhang, Y., Wall heating effect on local heat transfer in a rotating two-pass square channel with $90^{\circ}$ rib turbulators. Int. J. Heat Mass Transfer, 1994. 37(9): p. 1411-1420.

[32] Han, J.-C., Zhang, Y.-M., and Kalkuehler, K., Uneven Wall Temperature Effect on Local Heat Transfer in a Rotating Two-Pass Square Channel With Smooth Walls. J. Heat Transfer, 1993. 115(4): p. 912-920.

[33] Chanteloup, D., Juaneda, Y., and Bolcs, A. COMBINED 3D FLOW AND HEAT TRANSFER MEASUREMENTS IN A 2-PASS INTERNAL COOLANT PASSAGE OF GAS TURBINE AIRFOILS. in ASME Turbo Expo 2002. 2002. Amsterdam, The Netherlands.

[34] Liou, T.M., Chang, S.W., Hung, J.H., and Chiou, S.F., High rotation number heat transfer of a $45^{\circ}$ rib-roughened rectangular duct with two channel orientations. International Journal of Heat and Mass Transfer, 2007. 50(19-20): p. 4063-4078. 
[35] Han, J.-C., Park, J.S., and Lei, C.K., Heat Transfer Enhancement in Channels With Turbulence Promoters. J. Eng. Gas Turbines Power, 1985. 107(3): p. 628635.

[36] Han, J.-C. and Park, J.S., Developing heat transfer in rectangular channels with rib turbulators. Int. J. Heat Mass Transfer, 1988. 31(1): p. 183-195.

[37] Bonhoff, B., Parneix, S., Leusch, J., Johnson, B.V., Schabacker, J., and Bolcs, A., Experimental and numerical study of developed flow and heat transfer in coolant channels with 45 degree ribs. International Journal of Heat and Fluid Flow, 1999. 20(311-319).

[38] Wagner, J.H., Johnson, B.V., and Kopper, F.C., Heat Transfer in Rotating Serpentine Passages With Smooth Walls. Journal of Turbomachinery, 1991. 113: p. 321-330.

[39] Madabhushi, R.K. and Vanka, S.P., Large eddy simulation of turbulence-driven secondary flow in a square duct. Physics of Fluids, 1991. 3(11): p. 2734-2745.

[40] Liou, T.-M. and Chen, C.-C., LDV Study of Developing Flows Through a Smooth Duct With a 180 deg Straight-Corner Turn. J. Turbomachinery, 1999. 121(1): p. 167-174.

[41] Liou, T.M., Chen, C.C., and Chen, M.Y., Rotating Effect on Fluid Flow in Two Smooth Ducts Connected by a 180-Degree Bend. J. Fluid Eng., 2003. 125(1): p. $138-148$.

[42] Johnson, R.W. and Launder, B.E., Local Nusselt number and temperature field in turbulent flow through a heated square-sectioned U-bend. International Journal of Heat and Fluid Flow, 1985. 6(3): p. 171-180.

[43] Seban, R.A. and McLaughlin, E.F., HEAT TRANSFER IN TUBE COILS WITH LAMINAR AND TURBULENT FLOW. Int. J. Heat Mass Transfer, 1963. 6: p. 387-395.

[44] Iacovides, H., Jackson, D.C., Kelemenis, G., Launder, B.E., and Yuan, Y.M., Experiments on local heat transfer in a rotating square-ended U-bend. International Journal of Heat and Fluid Flow, 1999. 20(3): p. 302-310. 